\title{
Conference 2014:
}

\section{Promoting Today's Ideas for Tomorrow's Medicine: Pharmaceutical Science to Regulatory Science}

\author{
June 10-13, 2014 \\ Centre Mont Royal, Montréal, QC, Canada
}

A joint conference of:

Canadian Society for Pharmaceutical Sciences

\section{Canadian Chapter of Controlled Release Society}

\section{Conference Chair:}

Robert Young, Simon Fraser University, Vancouver, BC

Scientific Chairs:

Elizabeth Kwong, Kwong Eureka Solutions, Montréal, QC

Grégoire Leclair, Université de Montréal, Montréal, QC

Arshad Siddiqui, Paraza Pharma Inc., Montréal, QC

Conference Organizing Committee:

CSPS: Brian Foster, Elizabeth Kwong, Grégoire Leclair, Raimar Loebenberg, Robert Young

CC-CRS: Todd Hoare, Brian Amsden 


\section{Table of Contents}

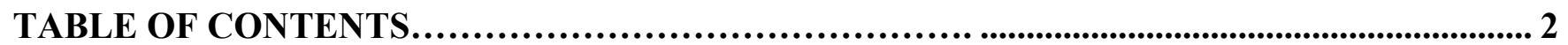

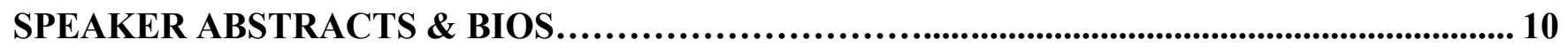

\section{BUILDING OPPORTUNITY DAY}

THE LIFE SCIENCES INDUSTRY IN CANADA: NOW AND THE FUTURE...

IRICoR: A Partnership Model with the Biopharmaceutical Industry .11

Steven J. Klein, Vice President, Business Development, IRICoR, Montreal, QC

Filling the Gaps to Shape the Future of Biopharmaceutical Research .11 Diane Gosselin, President and CEO, CQDM

The Centre for Drug Research and Development - Creating a Strategic Advantage and Opportunity for Canada. .12 Jason Crawford, Senior Director, Scientific Operations, CDRD, Vancouver, BC

Bridging Academic Research and the Need of the Industry for Safer and More Efficient Medicines............13 Max Felhmann, NeoMed Institute, Montréal, QC

Convergence and Collaboration: The Pivotal Role of Medical Technologies. .14 Diane Côté, Consortium MEDTEQ, Montréal, QC

\section{START-UP PHARMA: CASE STUDIES}

Life Science Start-up: Getting it Going .15

Peppi Prasit, Inception Sciences, San Diego, CA

Key Considerations for Efficient Development of New Molecular Entities (NME): Biotech and Clinical CRO Perspectives.

Marc Lefebvre, Algorithme Pharma, Montréal, QC,

Patrick Colin, gIcare Pharma Inc., Montréal, QC

\section{SUPPORTING INDUSTRY: FUNDING PANEL}

Introduction of Funding Opportunities for Innovation Set-up............................................................................17 Michelle Savoie, InVivo Montréal, Montréal, QC

Panel of Leading Venture Capitalists .

Daniel Hetu, Lumira Capital, Montréal, QC

Cynthia Lavoie, TVM Life Science Management Capital, Montréal, QC

Gerald Shipps, TB Drug Accelerator (a Bill \& Melinda Gates Foundation funded initiative), Boston, MA, USA 


\section{SPECIAL PRESENTATION BY DR. SUPRIYA SHARMA, HEALTH CANADA}

Facing Tomorrow's Regulatory Challenges Today

Supriya Sharma, MD, MPH, FRCPC, Acting Associate Assistant Deputy Minister, Health Canada

\section{GLAXOSMITHKLINE EARLY CAREER AWARD LECTURE}

Development of Intravaginal Microbicides for the Prevention of HIV Infection.

Emmanuel Ho, College of Pharmacy, Faculty of Health Sciences, University of Manitoba

\section{SESSION 1: EARLY DISCOVERY}

Discovery of VX-222: A Potent HCV NS5B Polymerase Inhibitor.

Sanjoy Kumar Das, Angiochem Inc.

Cross-institutional Drug Discovery Models: The OICR Experience Rima Al-Awar, Ontario Institute for Cancer Research (OICR)

Undruggable Means Undone

Michael Serrano-Wu, Director of Medicinal Chemistry, Therapeutics Projects Group, Broad Institute

The Discovery and Characterization of Orally Bioavailable Inhibitors of PTP 1B W. Cameron Black, Kaneq Pharma Inc.

\section{SESSION 2: LATEST TOPICS IN BIOEQUIVALENCE}

Expectations and Challenges in Small Molecules Regulated Bioanalysis

Adrien Musuku, Director, Biopharmaceutics, Pharmascience

NEW EMA Guidance for BE of Modified Release Products: Upcoming Challenges .24 Fethi Trabelsi, Vice-President, Scientific Affairs, Biopharma Services Inc.

Second Entry Respiratory Product Equivalence Requirements: A Comparison Between Jurisdictions .....25 Manon Belisle, Director Biopharmaceutics and Clinical Development, Teva Canada Limited

What is TPD Working On?

Eric D. Ormsby, Manager, Office of Science, Therapeutic Products Program, Health Canada

\section{PLENARY LECTURE 1}

Potential Applications of Human Stem Cells in Therapeutics Mickie Bhatia, Senior Scientist and Director of the McMaster Stem Cell and Cancer Research Institute (SCC-RI) McMaster University, Hamilton, ON

\section{SESSION 3: SUCCESSFUL CANDIDATE SELECTION FROM DRUGGABILITY TO REGULATORY SUBMISSION}

Physicochemical and Biopharmaceutical Characterization of Development Candidates - What do we Need to Know? .28 Elizabeth Vadas, InSciTech

Trainee Presentation: Synthesis, Preparation, and Characterization of Polymeric Nanocapsules of Nifuroxazide Analogues (Poster Abstract \# 60)

Michael Jefferson Amorim de Oliveira, University of Sao Paulo

Playing your Cards Hard and Early, But Smart: Advances in Increasing the Odds of Successful Development of Drug Candidates .29 Andrey Peresypkin, Director, Materials Discovery and Characterization, Vertex Pharmaceuticals, Boston, MA 
Believing is Seeing: Breakthrough Thinking in Pharmaceutics \& Drug Delivery

Riccardo Panicucci, Global Head of Chemical and Pharmaceutical Profiling (CPP) at Novartis

\section{SESSION 4: REGULATORY CONSIDERATIONS AND ANALYSIS OF SUBSEQUENT ENTRY BIOLOGICS}

Canada's Approach to Subsequent Entry Biologics (SEBs).

Agnes Klein, Director, Biologics and Genetic Therapies Directorate, Health Canada

Regulatory Considerations for Biosimilars in the United States.

Sue Lim, U.S. Food and Drug Administration, Center for Drug Evaluation and Research, Office of New Drugs

Analytical Tools for Characterizing Biologics

Steven A. Berkowitz, Consultant

Assessment of Biosimilarity and Interchangeability

Laszlo Endrenyi, Professor Emeritus, Pharmacology and Biostatistics, University of Toronto

\section{SESSION 5: NANOMEDICINE}

Strategies to Overcome Challenges Facing Nanomedicines .34

Christine Allen, Leslie Dan Faculty of Pharmacy, University of Toronto

Trainee Presentation: NMR and Fluorescence Studies of pH-sensitive Liposomes Based on a Molecular Switch (Poster Abstract \#22)

Warren Viricel, Université de Montréal

Phenylboronic Acid Modified Mucoadhesive Nanoparticles Facilitate Weekly Treatment of Dry Eye

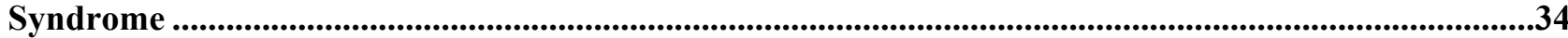

Frank Gu, Department of Chemical Engineering, University of Waterloo

Therapeutic Potentials of Carbohydrate Based Nanomedicine .35

Ravin Narain, Department of Chemical and Materials Engineering, University of Alberta, Edmonton, AB

\section{PLENARY LECTURE 2}

Current Paradigm of Cancer Nanomedicine and its Perspective - A Gap Between Experimental \& Clinical Worlds .37

You Han Bae, College of Pharmacy, University of Utah, Salt Lake City, Utah

SESSION 6: GOING BEYOND ORAL DELIVERY ... STRATEGIES TO ENABLE DRUGS TO REACH NEW TARGETS

Seven-Day Microneedle Enhanced Transdermal Delivery: Bench to Bedside Audra Stinchcomb, School of Pharmacy, University of Maryland, \& AllTranz Inc., Lexington, KY, USA

Fabricated Nano and Microparticles for Non-oral Delivery Brian Farrer, Principal Scientist, Liquidia

Trainee Presentation: RNAi-Based Nanomicrobicide for the Prevention of Male-to-Female Transmission of HIV-1 (Poster Abstract \#35)

Sidi Yang, University of Manitoba

Paradigm Shifts in Ocular Drug Delivery Heather Sheardown, Chemical Engineering, Faculty of Engineering. McMaster University, Hamilton ON

Designing and Engineering a Multipurpose Delivery Platform for Prevention and/or Treatment of Sexually Transmitted Infections. . .40 Jill Steinbach, Department of Bioengineering, University of Louisville

4s | Conference 2014 


\section{SESSION 7: NEW TRENDS IN MASS SPECTROMETRY FOR DRUG DISCOVERY \& DEVELOPMENT}

Mass Spectrometry in the Pharmaceutical Industry: Past, Present and Future .42 Lucinda Cohen, Director, NJ Discovery Bioanalytical Group, Merck Research Laboratories

Current Advancements in Large Molecule Bioanalysis by High Resolution Mass Spectrometry (HRMS) ..42 Fabio Garofolo, Vice President Bioanalytical Services, Algorithme Pharma

Trainee Presentation: Optimization of a PAMPA-BBB Model and Subsequent Validation by a Consolidated LC-MS/MS Method (Poster Abstract \#6).

Florian Bernard, Université de Montréal

Chemical Proteomics: Identification of Drug Targets and Mechanisms. Juergen Kast, The Biomedical Research Centre, University of British Columbia

Ion Mobility in Pharmaceutical Applications

Colin S. Creaser, Centre for Analytical Science, Department of Chemistry, Loughborough University, UK

\section{CSPS LIFETIME ACHIEVEMENT AWARD LECTURE}

Sense and Sensibility (?) in Science .45

Lasszlo Endrenyi, Professor Emeritus, University of Toronto

\section{CSPS AWARD OF LEADERSHIP IN CANADIAN PHARMACEUTICAL SCIENCES LECTURE}

A Bright Future for Pharmaceutical Sciences in Canada

Rav Kumar, Vice-President - R\&D Operations/ Business Development, GSK Canada

\section{SESSION 8: BIOMARKERS IN PHARMACOTHERAPY AND DRUG DEVELOPMENT}

Oxytocin as a Biomarker of Stress Disorders \& Relational Difficulties: Therapeutic Implications . .47 Kathleen Hegadoren, University of Alberta, Edmonton, $A B$

Trainee Presentation: Correlation between Arachidonic Acid Metabolites and Renin-angiotensin System Peptides in Adjuvant Arthritis Rat (Poster Abstract \#42)

Ali Aghazadeh-Habashi, University of Alberta

Biomarkers in Osteoarthritis and Rheumatoid Arthritis Roman Krawetz, Departments of Surgery and Cell Biology \& Anatomy, University of Calgary

Arachidonic Acid Metabolism Profile as Biomarker of Cardiac Function in Inflammatory Conditions: Arthritis.

Fakhreddin Jamali, Professor, Faculty of Pharmacy \& Pharmaceutical Sciences, University of Alberta

\section{SESSION 9: APPLICATION OF QUALITY BY DESIGN (QBD) AND LIFECYCLE MANAGEMENT} TO ANALYTICAL PROCEDURE

Implementation of Analytical Quality by Design Concepts at Pfizer .50 Loren Wrisley, Senior Director, Analytical Research and Development, Pfizer, Groton, CT

Trainee Presentation: Greyscale Inkjet Printing of Personalised Levothyroxine Doses and Optical Dose Verification (Poster Abstract \#72) .50

Mustafa Alomari, UCL School of Pharmacy

USP Stimuli Article on Lifecycle Management of Analytical Procedures .50 Elisabeth Kovacs, Chief Scientific Officer Chemistry and Analytical Sciences, Apotex

Implementation of QbD (Quality by Design) to Analytical Procedures - FDA Perspective .51 Sharmista Chatterjee, ONDQA/OPS/CDER/FDA 


\section{SESSION 10: CURRENT TOPICS IN DRUG SAFETY}

Principles of ADME-Tox in Drug Discovery and Development.

Leanne L. Bedard, Principal and Consultant, Bedard ADME-Tox Solutions

Assessment \& Prediction of Drug-Drug Interaction Potential at Drug Discovery \& Development Stages VX 222 Case Study

Jean-François Lévesque, DMPK Department, Vertex Pharmaceutical Canada, Laval, QC

Metabolic Activation and Reactive Metabolites

R. Scott Obach, Pharmacokinetics, Dynamics, and Drug Metabolism Department, Pfizer Inc., Groton, CT, USA

Genotoxicity Evaluation of the API and Impurities: Current ICH Guidelines. Robin M. Walker, CanBioPharma Consulting Inc.

\section{SESSION 11: RECENT TRENDS IN MATERIAL AND BIOMATERIAL RESEARCH}

Specific and Non-specific Interaction Forces at Electrified PEG-Functionalized Lipid-Bilayers ...................56 Markus Valtiner, Max Planck Institute, Germany

Polysaccharide-based Nanostructures for MRI Contrast Improvement . .56

V. Gaëlle Roullin, Pharmaceutical Nanotechnology Laboratory, Faculté de pharmacie, Université de Montréal

Towards Biomimetic Three-dimensional Micro-environments: Biomaterials and Microfabrication

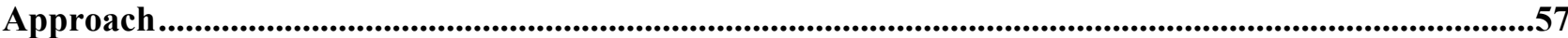
Shilpa Sant, Department of Pharmaceutical Sciences and Bioengineering, University of Pittsburgh

Towards the Development of Bioadhesive Polymers Mimicking Myelin Basic Protein .58 Xavier Banquy, Université de Montréal

\section{WEDNESDAY, JUNE 11}

\section{POSTER SESSION 1 - CSPS AND CC-CRS POSTERS}

\section{Biomedical Sciences}

1. The Effect of Advanced Age and Inflammation in Inducing Stroke in Hypertensive Situations ...........60

2. Novel Probiotic Dental Caries and Periodontal Disease Bio-Therapeutic .................................................60

3. Oxygen Delivery System for Tissue Growth .....................................................................................................61

4. The Effect of Local Delivery of Hypoxia Mimics on Biocemeraic Bone Graft Remodeling....................62

5. Study on the Potential Effect of Lactobacillus Reuteri in Inhibiting Colon Cancer Cells and the Role of Short Chain Fatty Acids Production: In-Vitro Analysis

Pharmaceutical \& Analytical Chemistry

6. Optimization of a PAMPA-BBB Model and Subsequent Validation by a Consolidated LC-MS/MS Method

7. Validation Method for the Quantitation of Seven Compounds used in Ophthalmology in Multiple Simulated Lachrymal Fluids Using Liquid Chromatography Tandem Mass Spectrometry ...................64

8. Development of Absolute Quantitative Analysis of Multiple Bile Acids Using LC/MS/MS....................65

9. Stability of Four Compounded Preparations of Tranexamic Acid and Alpha Thrombin ......................65

Pharmacokinetics \& Pharmacodynamics

10. Contribution of Pinocembrin Enantiomers to Potential Stereospecific Differential Pharmacokinetic 
and Pharmacodynamic Behaviour

11. Effect of Glycine on Cognitive Functions of 3xTg-AD Mice ......................................................................66

12. Pharmacokinetic Profile of a New High-Strength Fixed-Dose Combination of Ibuprofen/Methocarbamol 400/500 mg Tablet in Comparison to Single-component in the Presence and Absence of Food.

13. Coenzyme Q10 Lowers Blood Pressure and Heart Rate and Increases Red Blood Cell Concentrations of ATP in a Freely Moving Rat Model

Drug Delivery \& Pharmaceutical Technology

14. Efficacy of an Oral and Tropically Stable Lipid-based Formulation of Amphotericin B (iCo-010) in an Experimental Mouse Model of Systemic Candidiasis....

. .68

15. Investigating the Intraluminal Processing of Arylimidamide DB1960 in a Nanoparticle Lipid Formulation using an in vitro Lipolysis Model

16. Effect of Human Polynucleotide Kinase/Phosphatase Inhibitor on Survival of Acute Myeloid Leukemia Cells Bearing the Leukemic Stem Cell Phenotype CD123 ${ }^{+} / \mathrm{CD}{ }^{-} 1^{-}$Following Exposure to External Gamma Irradiation or Auger Electron Radioimmunotherapy.

17. Docetaxel-Loaded Polymeric Nanoparticles of PLGA for Sustained Release Intravenous Application: Preparation and In Vitro Characterization

18. A Comprehensive Study on the Freeze-Drying Process to Increase the Long-Term Stability of Polymeric, Drug-Loaded Nanoparticles

19. Chitosan-Based Nanogels in Macrogels aimed at the Controlled Release of Active Substances for the Local Treatment of Defective Cartilage

20. Fabrication and Characterization of a Segmented Reservoir Intravaginal Ring Device for Controlled Release of Hydroxychloroquine .72

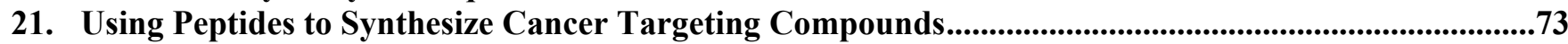

22. NMR and Fluorescence Studies of pH-sensitive Liposomes Based on a Molecular Switch ......................73

23. In vitro Comparison of the Efficiency of Levofloxacin-Loaded Polymeric Nanoparticles Versus Liposomes to Kill Pseudomonas Aeruginosa Developing in Cystic Fibrosis Patients...............................74

24. Microdialysis Procedure to Investigate the Extent of Neutralization of Palliative Calcium Gluconate Following Ophthalmic Hydrofluoric Acid Exposure

25. Preparation of Micelle-forming ABC Tri-Block Copolymers as Nano-reservoirs for Controlled Drug Release

26. Investigating the Structure of $\mathrm{pH}$-sensitive Lipids Based on a Molecular Switch .....................................76

27. Modeling the Absorption of Metformin in Patients with Post Gastric Bypass Surgery .............................77

28. Cyclodextrin-based Nanoparticles Overcome Drug Resistance to Antimelanoma Drug .........................77

29. Development of Nanostructured Hydrogel for Spatial and Temporal Controlled Release of Active Compounds

30. Co-Printing of Flexible Dose Combinations of Levothyroxine and Liothyronine for the Treatment of Hypothryroidism

31. Development of a New Controlled-Release Oxybutynin Tablet Using a Programmable Rupturable Film Coating Approach

32. Effect of Anti-Psoriatic Drugs on Pathological Skin Substitutes Produced by Tissue Engineering ........80

33. A Novel Therapeutic Textile Imprinted With Dermotex ${ }^{\circledR}$ Econazole-Loaded Microparticles ................80

34. Gene Profiling Characterization of a Psoriatic Skin Model ............................................................................81

35. RNAi-Based Nanomicrobicide for the Prevention of Male-to-Female Transmission of HIV-1 ..............81

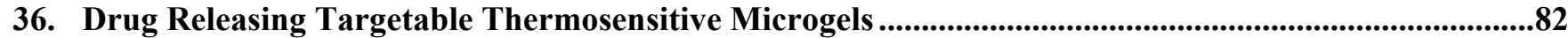

37. The Effect of Polymer Charge and Architecture on Protein Release Kinetics of Injectable, Degradable

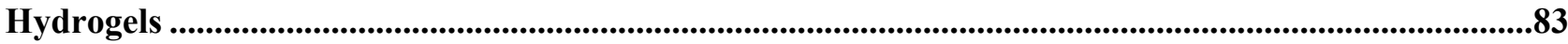

38. Injectable, In-situ Gelling, Cyclodextrin-dextran Hydrogels for the Partitioning-driven Release of

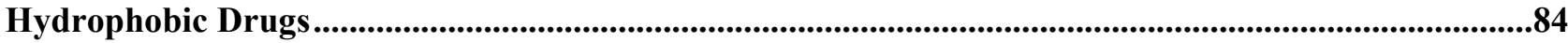

39. Engineered Solid Lipid Nanoparticles of Paclitaxel for Effective Management of Lung Carcinoma ....84 


\section{THURSDAY, JUNE 12}

\section{POSTER SESSION 2 - CSPS AND CC-CRS POSTERS}

\section{Biomedical Sciences}

40. Dietary Administration of Simvastatin Decreases Castration-Resistant Progression of the LNCaP

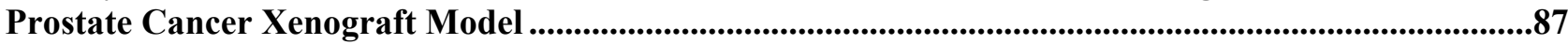

41. MyoNovin Synthesis, Purification, Analysis and Bioactivity on Muscle Fibers from Zebrafish ............88

42. Correlation between Arachidonic Acid Metabolites and Renin-angiotensin System peptides in

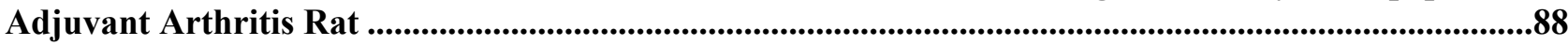

43. Significance of Bone Morphogenetic Proteins for Understanding of Traditional Chinese Herbs in Treatment of Liver Disease

44. Probing the efficiency and linkage specific cleavage activity of Sialidase in Micromonospora viridifaciens

Clinical Sciences \& Pharmacy Practice

45. In Search of the Optimal Strategy for Sedative Discontinuation: A Literature Review .........................90

46. Pharmacy Students-As-Immunizers: A Scoping and Jurisdictional Review .............................................91

47. Exposure to Rufinamide and Risks of CNS Adverse Events in Drug-Resistant Epilepsy: A Systematic Review and Meta-Analysis of Randomized Placebo-Controlled Studies

48. Cardiovascular Safety of NSAIDs: A Systematic Review of Meloxicam . .92

Pharmacokinetics \& Pharmacodynamics

49. Cytochrome P450 Metabolites of Arachidonic Acid in Plasma \& Heart as Bio-Marker of NSAIDS Induced Cardiovascular Risk in Adjuvant Arthritis Rat.

50. Stereospecific Characterization of the Pre-Clinical Pharmacokinetics, Pharmacological Activities and Content Analysis in Natural Health Products of 8-Prenylnaringein, A Potent Phytoestrogen Found in Hops (Humulus Lupulus)

Pharmaceutical \& Analytical Chemistry

51. Hepatoprotective Properties of Commiphora Opobalsamum (Balessan), A Traditional Medicinal Plant of Saudi Arabia

52. Chemical and Biological Study of Flueggea virosa Native to Saudi Arabia............................................95

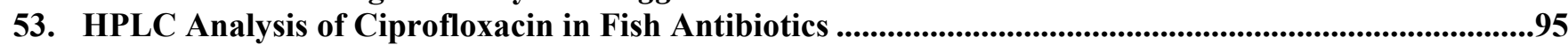

54. Carboxymethyl and Hydrazide Functionalized $\beta$-Cyclodextrin Derivatives: A Systematic Investigation of Complexation Behaviors with the Model Hydrophobic Drug Dexamethasone. . .96

Drug Delivery \& Pharmaceutical Technology

55. Amino Acid Functionalized Nanodiamonds as Gene Delivery Vectors................................................96

56. Trastuzumab Modified Targeted PLGA Nanoparticles for HER2 Positive Breast Cancer.....................97

57. The Impact of Implementation of the Canadian Regulatory Requirements on the Quality of Natural Health Products: The Glucosamine Case.

58. In Silico Modeling (Gastroplus) to Overcome Pharmacogenomic Variation in Human Using Different Dextromethorphan Dosage Forms.

59. Validation of a Molecular Dynamic Model: PEGylated Liposome Surface Interacting with Salt Ions of the Bloodstream

60. Synthesis, Preparation, and Characterization of Polymeric Nanocapsules of Nifuroxazide Analogues 99 
61. A Microinjection Device for Administering In Situ-Gelling Hydrogels for Ophthalmic Drug Delivery 100

62. Characterization of a Novel Surface-coated Matrix Intravaginal Ring Device for the Sustained Release of Hydroxychloroquine 100

63. Drug Release System Based on an Inorganic Phosphate Hydrogel with Extreme Thixotropic Behavior 101

64. Scavenger Receptor Class B Type I (SR-B1) Expression Levels Modulate CPX-351 Uptake within Cancer Cells .102

65. Targeting CD205 receptor as an Approach to Develop a Cancer Vaccine ..........................................102

66. Application of Super Fine Powder, Special Low Viscosity Grade of Hydroxypropyl Cellulose (HPCSSL-SFP) as a Tablet Hardness Enhancer for Hydrophilic Matrix Formulation ...................................103

67. Application of Hydroxypropyl Cellulose to Solubility Enhancement of Poorly Soluble Drugs.............103

68. Relationship between PEG Surface Coverage-density and the Physico-chemical Properties of Polymeric Nanoparticles 104

69. Catechol-Chitosan/Genipin Mucoadhesive Hydrogel for Buccal Drug Delivery: An In Vivo Study ...105

70. Omega-3 Tablets Based on CarboxyMethyl Starch 105

71. Inkjet Printing of Therapeutic Warfarin Doses, How Printing can Address Clinical Needs of Patients 106

72. Greyscale Inkjet Printing of Personalised Levothyroxine Doses and Optical Dose Verification .106

73. Challenges and Opportunities to use Biowaivers to Compare Generics in China .107

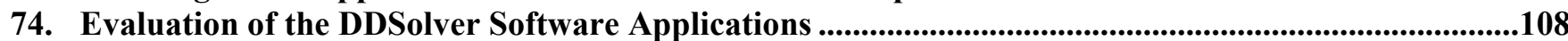

75. Biorelevant Membranes as in vitro Models for the Evaluation of Drug Permeability ............................108

76. Surface Properties and Interactions of pH Sensitive Polymeric Nanoparticle Monolayers...................109

77. Effect of Polymer Architecture on Hydrophobic Drugs Encapsulation and Release from Polymeric Nanoparticles 109

78. Use of Heat-activated Thermosensitive Liposomes of Cisplatin (HTLC) for the Treatment Cervical Carcinoma .110

79. Folate Receptor-Targeted Pluronic Nanoparticles for Ovarian Cancer Gene Therapy .........................111

80. Tunable Growth Factor Delivery from Injectable Hydrogels for Central Nervous System Repair.....111 


\title{
Speaker Abstracts
}

\author{
\& Bios
}




\section{Tuesday, June 10}

\section{Building Opportunity Day}

\section{The Life Sciences Industry in Canada: Now and the Future...}

\section{IRICoR: A Partnership Model with the Biopharmaceutical Industry}

Steven J. Klein, Vice President, Business Development, IRICoR, Montreal, QC

IRICoR, the Institute for Research in Immunology and Cancer-Commercialization of Research, is a not-for-profit drug discovery and commercialization centre created in 2008 and based at Université de Montréal's Institute for Research in Immunology and Cancer (IRIC). Our vision is to efficiently capture and maximize the value of innovative academic research and to rapidly translate this into new therapies for unmet medical needs, in partnership with the biopharmaceutical industry. IRICoR is a fully-integrated drug discovery centre, with the largest academia-based, industryexperienced medicinal chemistry group in Canada. IRICoR's strategy focuses on leveraging its deep scientific and business knowledge in oncology and related fields to select and invest in highly innovative projects, thus filling the innovation translation gap and accelerating the commercialization of novel therapies and technologies. IRICoR has a number of ongoing major drug discovery partnerships with companies such as Bristol-Myers Squibb, Pfizer, Merck, Pharmascience, and Domain Therapeutics.

\section{Steven J. Klein}

Dr. Steven Klein is IRICoR's VP, Business Development, with over 15 years of experience in a variety of fields, including oncology, vaccines, and CNS-related indications. Prior to joining IRICoR, Dr. Klein held Business Development roles at Labopharm, Neurochem/BELLUS Health and the Canadian Network for Vaccines and Immunotherapeutics (CANVAC). During his career, Dr. Klein negotiated and concluded a wide range of licensing and co-development agreements, covering both products and technologies, with industry leaders such as Pfizer, Bristol-Myers Squibb, Merck,
Roche, GSK, Domain Therapeutics, Grunenthal, Nippon Shinyaku, BD Biosciences, and MDS Pharma Services.

Dr Klein holds a B.Sc. (Honors) in Chemistry from Concordia University, a Ph.D. in Experimental Medicine from McGill University, and an MBA from Queen's University. Dr. Klein is a Board member of the Alliance for Commercialization of Canadian Technologies and BioTalent Canada.

\section{Filling the Gaps to Shape the Future of Biopharmaceutical Research}

Diane Gosselin, Ph.D., MBA, President and CEO, CQDM

The pharmaceutical industry is the most researchintensive global industrial sector with over $\$ 135$ billion annually invested into drug discovery, development and commercialization. Annual R\&D spending by the industry is five times greater than that of the aerospace \& defense industries, 4.5 times more than the chemicals industry, and 2.5 times more than the software and computer services industry. Moreover, no other industrial sector consistently invests more in $R \& D$, even in times of economic turmoil and financial crisis. Yet, over the last decade, the industry has faced a productivity crisis in R\&D attributed to many factors.

This gap in innovation has led to the introduction of new R\&D models, such as CQDM, in an attempt to address this productivity crisis. How do we bring pharmaceutical companies to the same table to express what their common needs are and have them co-invest in solutions in order to reduce the risk of development, $R \& D$ costs and time to market? How do we best seek innovation, new therapeutic approaches and new research avenues? How do we keep SMEs and academic researchers aligned with the needs of the pharmaceutical industry and those of patients? How do we get all the actors of this ecosystem to invest time and money for the good of the patient population? 
This presentation will go over CQDM's unique business model and how it addresses these questions, as well as the value is has created for all actors in the biopharmaceutical R\&D ecosystem (pharmas, biotechs, academia, governments, patients) to fill the gaps and shape the future of biopharmaceutical research in Canada.

\section{Diane Gosselin}

Diane Gosselin was nominated President and CEO of CQDM in November 2012. She joined the organization in 2008 as Vice President, Research and Business Development where she was responsible for establishing all funding programs and managing the corporate development activities. She has twenty years of experience in research and corporate financing, namely at the Fonds de solidarité FTQ where she helped several companies implement their drug development strategy and was actively involved in the elaboration of a strategic initiative aimed at promoting innovation in the life sciences sector. She took part in several technological maturation matters, notably as a board member of the Bio-Innovation Funds (2005-2008). She also served as a board member of Cardianove and the Biotechnologies Oceanova (2005-2008). At PROCREA BioSciences, she led the research team which created and developed the first non-invasive diagnostic test for endometriosis. Mrs. Gosselin holds a Ph.D. in microbiology and immunology from the Université de Montréal, as well as an Executive MBA from the Université du Québec à Montréal and the Université Paris-Dauphine.

\section{The Centre for Drug Research and Development - Creating a Strategic Advantage and Opportunity for Canada}

Jason Crawford, Ph.D., Senior Director, Scientific Operations, CDRD, Vancouver, BC

The development of novel therapeutic discoveries is key to Canada's future prosperity; but in order to capitalize on their value, it is critical to devote resources to the translation of our collective innovation into real commercial products. A unique national drug development engine has therefore been created in Canada to transform scientific innovation into commercialization impacting human health: The CDRD/CVI Enterprise (comprised of The Centre for Drug Research and Development (CDRD) and its commercialization vehicle, CDRD Ventures Inc.
(CVI)). As Canada's fully-integrated national drug development and commercialization centre, the single mandate of this Enterprise is to collaboratively validate and add value to cuttingedge, promising discoveries from leading academic institutions across the country, thereby presenting de-risked and viable investment opportunities for the private sector.

To achieve this, CDRD/CVI has established over the past seven years, state-of-the-art drug development infrastructure, scientific and business expertise. This has been coupled with and supported by unique partnerships with industry, academia, foundations and government that has established a diverse funding base, provided opportunities to leverage investments, and ensured early industry participation and commercial pull for the technologies. CDRD/CVI is thus able to provide a competitive advantage for Canadian researchers by providing collaborative de-risking and advancement of technologies in a rigorous and peer-reviewed manner, enabled by leveraged funding on a projectby-project basis.

Through CVI, the Enterprise can then also facilitate the creation of new spin-out companies, technology out-licensing or joint ventures. CVI's share of resulting profits flow back to CDRD to support future drug development projects, and the ongoing sustainability of Canada's innovation engine.

\section{Jason Crawford}

As the Senior Director of Scientific Operations at The Centre for Drug Research and Development (CDRD), Canada's fully-integrated national drug development and commercialization centre, Dr. Crawford plays a key role in the evaluation of potential drug development projects to be incubated within CDRD, and holds responsibility for the successful execution of all subsequent project development plans, and thus the overall management of CDRD's scientific resources. Prior to assuming this role, he was Head of Medicinal Chemistry at CDRD, having joined the organization in 2008.

Previously, from 1999-2007, he held a series of increasingly-senior positions at AnorMED Inc. initially a Medicinal Chemist, and eventually the Manager of the Development Chemistry Group, leading the synthetic development of all lead and candidate compounds intended for use in pre-clinical and clinical trials. Notably, for plerixafor, which now generates over $\$ 150$ million annually in global sales as Mozobil, he oversaw the technical transfer 
of the development process to a contract research organization, the subsequent clinical manufacture, and the eventual validation of the manufacturing process for commercial use. He also acted concurrently as an interdisciplinary project team leader for a clinical-stage anti-HIV therapeutic. In addition to his experience at AnorMED, Dr. Crawford also developed novel antibacterial agents in his role of Senior Scientist, Medicinal Chemistry at Microcide Pharmaceuticals Inc. based in Mountain View, California.

Dr. Crawford received his B.Sc. in chemistry/biochemistry in 1991, and a Ph.D. in organic chemistry in 1996 from the University of Victoria (Canada). He then conducted post-doctoral research in organic synthesis at the Ohio State University from 1996-1997.

\section{Bridging Academic Research and the Need of the Industry for Safer and More Efficient Medicines}

Max Felhmann, NeoMed Institute, Montréal, QC

The development of a new therapeutic drug is a complex, lengthy and expensive process. The academic world plays a key role in identifying molecular targets on which drugs act, but usually do not have the capacity to make the medicines acting on these targets act. This is typically done by pharmaceutical companies. Today however, to lower their business risks, companies reduce their internal research and development efforts and increasingly seek to acquire projects from external sources. Therefore a gap exists between the ability of academia to deliver innovative drug targets and the need of Pharma industry for projects that include a mature candidate drug. NEOMED aims to bridge academic research with the real needs of the BioPharma industry and investors of the life science sector in order to deliver needed treatments to patients and the society. Created in November 2012 as a Public-Private not-for-profit organization, NEOMED identifies, finances and implements innovative drug discovery projects to bring them up to human proof-of-concept. Our business model is structured around an internal multidisciplinary team of industrial experts in drug discovery that outsource the operational activities to the most qualified partners. The mature and high-quality assets developed will then be sold to downstream partners, be the basis for the creation of solid spin-offs or be used to consolidate the commercial performance of existing companies. All the proceeds will be reinvested in the NEOMED fund to enable activity on more projects. NEOMED is hosted in its own facility, the NEOMED Institute, an open-access life science hub dedicated to drug discovery and development. Today, the Institute is hosting 20 independent BioPharma firms - employing more than 130 scientists - including Contract Research Organizations, consulting, legal firms and Biotech companies. NEOMED and the NEOMED Institute is a pole of growth for the Canadian life sciences sector, contributing directly to translate early-stage discoveries from the vast reservoir of Canadian academic and Biotech innovations into economic prosperity and improved health outcomes.

\section{Max Fehlmann}

As President and CEO, Max Fehlmann is leading the entire NEOMED initiative. He is in charge of the strategic planning and corporate development of both NEOMED and the NEOMED Institute. During the last 35 years, the career of Dr. Fehlmann encompassed the 3 fundamental domains involved in NEOMED, from academic research (Forgarty International Fellow at Harvard Medical School, Professor of Pharmacology at the University of Nice in France, Research Director at INSERM in France, with over 200 peer reviewed publications), SME management (founder and $\mathrm{CEO}$ of Aster Biotechnologies in France and CEO of Chronogen in Montreal) and Pharma R\&D (VP R\&D for Zambon Group in Italy for all pre-clinical, clinical and licensing activities of the group worldwide as well as founder of Z-cube, a corporate Venture fund linked to Zambon Group).

Max obtained his $\mathrm{PhD}$ in Biochemistry from Université Laval in 1975, a Doctorat ès Sciences from the University of Nice (France) in 1981 and an MBA from HEC-CPA at Sophia Antipolis (France) in 1992. He received numerous scientific and business awards during his career in Europe and in Canada. 


\section{Convergence and Collaboration: The Pivotal Role of Medical Technologies}

Diane Côté, Consortium MEDTEQ, Montréal, QC

[Abstract not available]

\section{Diane Côté}

Diane Côté is President and CEO of the Consortium for industrial research MEDTEQ, launched in January 2013. MEDTEQ's objective is to facilitate and accelerate the development of innovative medical technologies. Ms. Côté was previously Vice-President - Quebec, of MEDEC, the national association created by and for the Canadian medical technology industry.

She is a member of the Advisory Board of the Bachelor Program in Biomedical Engineering and of the Méditis Training Program of the École Polytechnique de Montréal.

Previously, Ms. Côté was President and Chief Executive Officer and a member of the Board of Directors of a medical technology SME specialising in the use of state-of-the art mathematical modeling and neural networks for risk management purposes in obstetrics. She spearheaded the development and marketing of the company's products as well as its launch on the public markets.

Ms. Côté worked at IBM as member of the Sales and Communication management team. In addition, as Partner responsible for business development at Innovitech, a consulting company specialising in new technologies and in the development of innovative economic models, she lead numerous mandates in strategic planning, financing and partnerships.

Ms. Côté has also served on many boards, namely the Centre de recherche informatique de Montréal (CRIM), the Association des industries des technologies de la Santé (AITS) and InVivo, the life sciences and health technologies cluster of Metropolitan Montréal. She also served on the board of a number of charitable organisations in Québec.

Ms. Côté has a Bachelor's degree in Translation from the Université de Montréal 


\section{Tuesday, June 10}

\section{Building Opportunity Day}

\section{Start-up Pharma: Case Studies}

\section{Life Science Start-up: Getting it Going}

Peppi Prasit, Inception Sciences, San Diego, CA a

The traditional biotech hubs on the East Coast and West Coast have always been able to attract talent and capital, but innovation isn't restricted to San Diego, Boston or San Francisco. In 2011 we founded Inception Sciences based in San Diego following the acquisition of our previous company Amira Pharmaceuticals by Bristol Myers Squibb. Recently we created Inception Sciences Canada based in Vancouver. We plan on launching a similar unit in Montreal in the coming year tapping into the strong talent pool that exists locally. The talk will share our experience in creating these entities.

\section{Peppi Prasit}

Peppi Prasit has more than 25 years of experience in pharmaceutical research. $\mathrm{He}$ is a founder of Inception Sciences, a small molecule drug discovery company which has laboratories in San Diego and Vancouver, BC.

Prior to Inception Sciences, Peppi was cofounder of Amira Pharmaceuticals, Inc., a pharmaceutical company focused on the discovery and early development of new small molecule drugs. Amira successfully created five candidates entering clinical trials within five years from company inception. Amira was acquired by Bristol Myers Squibb in 2011.

Prior to Amira, Peppi spent 20 years as a scientist at Merck Frosst Canada and Merck Research Labs in San Diego.

Peppi received his undergraduate degree from University College London in the UK, Ph.D. from Victoria University of Wellington in New Zealand and served as a post-doctoral fellow at Princeton University.

\section{Key Considerations for Efficient Development of New Molecular Entities (NME): Biotech and Clinical CRO Perspectives}

Marc Lefebvre, Algorithme Pharma, Montréal, QC, Patrick Colin, gIcare Pharma Inc., Montréal, QC

With the recent changes in drug discovery and clinical models, collaborations between key stakeholders are critical for generating efficient development programs. This session will present several considerations from an early stage clinical CRO \& Biotechnology perspectives which are used for developing new drugs and efficient trials. Design factors include sponsor objectives, regulations, type of compounds and timelines. However, additional factors such as study population, medical expertise, safety events and communication can have a significant impact on study conduct. A case study analysis will be used to discuss the development of efficient programs while accounting for key stakeholder collaborations and design considerations.

\section{Marc Lefebvre}

Dr. Marc Lefebvre is the co-founder and the VicePresident, Scientific \& Regulatory Affairs at Algorithme Pharma. He leads scientific, regulatory and pharmacological decisions on the conduct of Early Stage Clinical Trials (Phase I/IIa) including bioavailability and bioequivalence studies. With more than 19 years of experience at Algorithme Pharma, he also worked at Schering Canada (1987), Marion Merrell Dow Canada (1994) and GlaxoWellcome (1995). Dr. Lefebvre had always interests in the development of new drugs and formulations (Phase I/IIa) and he still gives frequent lecturing at symposiums and workshops on the conduct of Phase I Clinical Trials and as an invited professor at the University of Montreal. Following his bachelor degree in Pharmacy, he completed his M.Sc. and Ph.D. degrees in Pharmacology (Faculty of Medicine) at the University of Montreal. He is member of the Board at Algorithme Pharma and his 
is also involved in different organisations related to the development of Health\&Science in the Montreal area.

\section{Patrick Colin, B.Pharm., Ph.D.}

Dr. Colin is a co-founder of gIcare pharma where he is responsible for the overall strategic and operational drug development process, including preclinical and clinical development activities. He is also the president of PCC Inc., a Drug Development Consulting firm offering overall, strategic advice to a variety of pharmaceutical and academic clients in North America and Europe. Previously, he spent more than 12 years at Axcan Pharma (today Aptalis Pharma), where he established and led the drug development department as the Vice-President, R\&D. His credentials include 26 years of global drug development experience, and more than 15 market approvals (of which 12 were related to GI) in the US, EU and Canada. Patrick Colin graduated from the Faculty of Pharmacy at the University of Montreal, where he obtained both a professional Pharmacy degree (B.Pharm.) as well as a Ph.D. in pharmacology.

\section{Session Chair}

\section{Elizabeth Kwong, Kwong Eureka Solutions, Montréal, QC}

Dr. Kwong retired from Merck after 23 years of service and established her own company, Kwong Eureka Solutions. She consults for small start-up companies and specialty drug products. She is also part of the Canadian Society of Pharmaceutical Sciences Board of Directors and is serving as a scientific advisor for the Journal of Pharmacy \& Pharmaceutical Sciences. Before retiring she was Senior Scientific Director at Merck \& Co. Basic Pharmaceutical Sciences (BPS) a subdivision of the Department of Pharmaceutical Sciences and Clinical Supplies (PSCS). She is also an adjunct professor in the Dept of Pharmaceutics at the University of Montreal and Dept of Chemistry at Concordia University. She received her B.S. Pharmacy (1980) and Ph.D. degree (Pharmaceutical Chemistry -1984) from University of British Columbia, Canada. She completed a postdoctoral fellowship in Pharmaceutics at the School of Pharmacy, University of Washington in Seattle (1984-1986). She then joined Cantest Laboratories, a contract facility in Vancouver, Canada as a Research Racing Chemist in the Race Horse Drug Screening Lab. Her next move was to Merck where she was part of several functional groups within Merck \& Co., starting from the Pharm Science group in Merck Frosst Canada Ltd in Montreal, Canada to the Merck Research Laboratory in Rahway, NJ, Boston, MA and West Point in PA. Elizabeth is currently located in Montreal, Canada. 


\section{Tuesday, June 10}

\section{Building Opportunity Day}

\section{Supporting Industry: Funding Panel}

\section{Introduction of Funding Opportunities for Innovation Set-up}

Michelle Savoie, InVivo Montréal, Montréal, QC

\section{Michelle Savoie}

Mrs. Michelle Savoie MBA, MPH, PhD, LL.M is the Chief Executive Officer of Montréal InVivo. She is also an Assistant professor, practical training at the Faculty of Pharmacy of the Universite de Montréal.

Mrs. Savoie brings to the organization over 20 years of experience in the health field and pharmaceutical sector. She has held strategic positions at various Pharma companies, including Schering Canada, Eli Lilly Canada and BristolMyers Squibb.

In addition, she currently sits on several boards and committees, including those at Université de Montréal, INESSS as well as the boards of directors of the Quebec Network for Personalized Healthcare (QNPHC) and the Personalized Medicine Partnership for Cancer (PMPC).

Mrs. Savoie was actively involved in the creation of the Quebec Consortium for Drug Discovery, the Quebec Personalized Health Care Strategy and Néomed.

A biochemist by training, Mrs. Savoie also holds an MBA from the University of Ottawa, a Masters in Public Health (MPH) from Boston University, a $\mathrm{PhD}$ in biomedical sciences from Université de Montréal as well as a Masters in Law and Health policy (LL.M.) from the Université de Sherbrooke.

\section{Panel of Leading Venture Capitalists}

Daniel Hetu, Lumira Capital, Montréal, QC

Daniel has more than 20 years of financing, mergers and acquisitions, and business development experience.

In 2005, Daniel joined Lumira Capital, one of the leading Canadian life science venture funds with offices in Montreal, Toronto and Boston.

Before joining Lumira Daniel was VicePresident Corporate Development at Biochem Pharma and Vice-President, business development North America at Shire following its acquisition of Biochem Pharma in 2001. During this period, Daniel completed several transactions (financing ,M\&A, licensing) in the therapeutic, vaccine and diagnostic sectors.

Prior to joining Biochem Pharma, Daniel was an investment banker at a major Canadian investment bank where he was involved in M\&A and financing transactions across various industrial sectors.

Daniel holds and an MBA from École des Hautes Études Commerciales (Montréal, Québec) and an M.D. from Université de Sherbrooke (Québec). 
Cynthia Lavoie, TVM Life Science Management Capital, Montréal, QC

Dr. Lavoie is a General Partner with TVM Life Science Management Inc. She is located in Montreal and is responsible for the investment strategy in Canada and the management of portfolio companies in the US and Canada. Cynthia serves on the boards of Anchor Therapeutics and Kaneq Bioscience. She was previously with VG Partners, a large Canadian private equity firm, where her most recent role was as Partner and head of life sciences. She served on the boards of investee companies Aegera Therapeutics, Cytochroma, Interface Biologics, Trillium Therapeutics and Zelos Therapeutics, among others. During her tenure, Cynthia had a direct role in the sale of VisualSonics, an imaging company, to SonoSite Inc., and Targanta Therapeutics to The Medicines Company.

Prior to joining VG Partners, Cynthia was a marketing strategy consultant with public company Vasogen (acquired by Intellipharmaceutics) and an Investment Program Manager with the Ontario Genomics Institute seeding early stage investments. An accomplished academic, Dr. Lavoie is an active participant on proof of principal granting committees for regional and national commercialization grants. She has ten years of research experience at McGill University and the University of Texas Southwestern Medical Center as well as an MBA from the Rotman School of Management.
Gerald Shipps, TB Drug Accelerator (a Bill \& Melinda Gates Foundation funded initiative), Boston, MA, USA

After graduating from the Massachusetts Institute of Technology with a $\mathrm{PhD}$ in Organic Chemistry in 1997, Gerald joined the Cambridge-based biotech startup company NeoGenesis as one of its first scientists. Gerald rose to the position of Sr. Director of Chemistry and External Collaborations; where he was involved in research activities with numerous pharma and biotech organizations. Following Schering-Plough's acquisition of NeoGenesis in 2005 he became the Director of Lead Discovery at the Cambridge site and advanced multiple novel series in oncology, inflammation, and infectious disease programs. Following the merger of Schering-Plough with Merck he assumed a broader role supervising a multidisciplinary group of screening, informatics, and medicinal chemistry capabilities. In 2012 Gerald joined the Bill \& Melinda Gates funded TB Drug Accelerator as the Medicinal Chemistry Lead and Program Manager, a dynamic role coordinating research between multiple pharmaceutical and academic members. 


\section{Wednesday, June 11}

\section{Special Presentation by \\ Dr. Supriya Sharma, Health Canada}

\section{Facing Tomorrow's Regulatory Challenges Today}

Supriya Sharma, MD, MPH, FRCPC, Acting Associate Assistant Deputy Minister, Health Canada

Dr. Sharma took on the role of Acting Associate Assistant Deputy Minister of the Health Products and Food Branch (HPFB) in February 2014. This was in addition to her responsibilities in the newly created Senior Medical Advisor role in HPFB since March 2013. Prior to that, Dr. Sharma has held a number of positions in Health Canada over the past decade in both the pre-market and post-market health product regulatory areas including most recently Director General of the Therapeutic Products Directorate, which had the regulatory responsibility for pharmaceuticals (prescription and non-prescription) and medical devices. She has also worked as a Senior Policy Advisor as part of the National Pharmaceuticals Strategy in Health Canada. Recently, she has returned to Health Canada following a leave of absence to work in an academic research group focusing on health innovation adoption in the Canadian Health system.

Trained as a pediatrician in both Canada and Australia, Dr. Sharma was a research fellow in hematology focused on clinical research relating to thalassemia and sickle cell disease and has worked on a number of large multi-centre clinical studies, including research in collaboration with Oxford University on a project in Sri Lanka. She then went on to complete a Masters of Public Health at the Harvard School of Public Health with a concentration in International Health and an interest in Health Policy. 


\section{Wednesday, June 11}

\section{GlaxoSmithKline Early Career Award Lecture}

\section{Development of Intravaginal Microbicides for the Prevention of HIV Infection}

Emmanuel Ho, Ph.D., College of Pharmacy, Faculty of Health Sciences, University of Manitoba

Despite successful human immunodeficiency virus (HIV) prevention strategies such as condom use, monogamy, and abstinence, in 2011 HIV has affected $>34$ million people globally. The region of Sub-Saharan Africa is especially affected, with $\sim 60 \%$ of women living with HIV. Women are biologically two times more likely than men to become infected with HIV through unprotected heterosexual intercourse. Some of the reasons include the larger surface area of the vagina/cervix in comparison to the male penis and the fact that after sexual intercourse, semen remains within the female genital tract for prolonged periods of time, increasing exposure to HIV. Ever since the identification of HIV as the etiological agent for AIDS in 1984, researchers around the world have desperately attempted to develop an effective vaccine but have been unsuccessful. Some of the reasons include the fact that conventional vaccines are meant to protect against disease and not infections and HIV infection can remain latent for long periods of time before causing AIDS. These concerns along with the lack of understanding of the virus make vaccine development difficult.

In the absence of an effective vaccine, development of preventative technologies such as microbicides (products designed for vaginal or rectal application for the purpose of reducing sexually transmitted infections) is an excellent alternative to condoms and remains a priority for combating the spread of HIV/AIDS. Our research group is interested in developing polymeric intravaginal rings (IVRs) as a microbicide platform for the sustained controlled release ( $>14$ days) of various bioactive molecules. IVRs have been shown to be safe, relatively simple to use without the assistance of a healthcare professional, and IVR replacement is infrequent (in comparison to daily vaginal gel applications). Our research team is also currently developing nanomicrobicides for the delivery of siRNA to target host cells (e.g. CD4+ immune cells) as a strategy for protecting against HIV infection. For siRNA to be developed into an effective vaginal microbicide, it must overcome the adhesive properties of cervicovaginal mucus, the acidic nature of cervicovaginal fluid (CVF; $\mathrm{pH}$ 3.5-4.9) and the presence of nucleases in CVF, all of which may contribute to the degradation of siRNA. As a result, we are developing nanoparticle formulations that will enhance targeted delivery and improve therapeutic efficacy.

\section{Emmanuel Ho}

Dr. Emmanuel Ho joined the University of Manitoba, College of Pharmacy as an Assistant Professor in 2010. He is also cross-appointed to the Faculty of Engineering, Department of Biomedical Engineering and the College of Medicine, Department of Immunology. He earned his Ph.D. in Pharmaceutical Sciences from the University of Toronto and was awarded numerous scholarships, most notably, an Industrial PostGraduate Scholarship from the Natural Sciences and Engineering Research Council of Canada (NSERC). As a post-doctoral fellow at the British Columbia Cancer Research Center, Dr. Ho was awarded the Canadian Institutes of Health Research (CIHR) PostDoctoral Fellowship along with the Michael Smith Foundation for Health Research Post- Doctoral Fellowship (MSFHR). His current research interests include the development and characterization of novel medical devices, vaccines, and drug delivery strategies for the treatment and prevention of HIV/AIDS. He was recently presented the $2013 \mathrm{Rh}$ Award for Outstanding Contributions to Interdisciplinary Scholarship and Research. Dr. Ho's research program is supported by grants from the Bill and Melinda Gates Foundation, CIHR, Canada Foundation for Innovation (CFI), Manitoba Health Research Council (MHRC), Manitoba Medical Service Foundation (MMSF), and the Dr. Paul Thorlakson Foundation Fund. 


\section{Wednesday, June 11 \\ SESSION 1: \\ Early Discovery}

\section{Discovery of VX-222: A Potent HCV NS5B Polymerase Inhibitor}

Sanjoy Kumar Das, Angiochem Inc.

Infection by the hepatitis $\mathrm{C}$ virus (HCV) has been recognized as one of the leading causes of liver impairment such as cirrhosis and hepatocellular carcinoma. The positive strand RNA virus of the Flaviviridae family chronically infects approximately $3 \%$ of the world population or 200 million individuals. HCV infection is the leading cause of liver transplantation in the United States and is responsible for almost 10,000 deaths per year. For a decade, PEG-INF in combination with ribavirin was the standard of care and was poorly tolerated with limited efficacy against the most prevalent genotype 1 . Less than $50 \%$ of the patients treated would achieve sustained virological response (SVR: undetectable HCV RNA levels 6 months after completion of treatment) and furthermore, treatment was poorly tolerated accompanied by side effects such as anemia and flu-like symptoms. As a result, recently a three-drug combination of PEG-IFN, RBV plus one of two approved direct acting antivirals (DAA), Incivek ${ }^{\mathrm{TM}}$ or Victrelis $^{\mathrm{TM}}$ has become the current standard of care. These new DAAs act as reversible covalent inhibitors of the NS3/4A protease. When combined with PEGIFN/RBV, either two of these agents can significantly increase sustained virological response (SVR) and dramatically reduce treatment duration. However, new treatments with all-oral regimens that would eliminate the need for intravenous PEG-IFN are desirable and a tremendous effort has been focused to two other targets that include inhibitors of the non-structural NS5A protein and nucleoside/nonnucleoside inhibitors of the HCV RNA dependent RNA polymerase ( $R d R p)$. The $R d R p$ is one of the most studied targets because it has been shown that a functional polymerase is essential for viral replication. As a result, Gilead has launched a nucleotide analog polymerase inhibitor sofosbuvir (brand name Sovaldi) in December 2013 and several others are also in clinical trials (VX-135, ABT-072,
ABT-333, BMS-791325). We have described the identification and SAR studies of novel thiophene analogs which bind to the thumb site as $\mathrm{HCV}$ polymerase inhibitors. In our continued efforts in this series, we have identified VX-222 as one of the most potent $\mathrm{HCV}$ polymerase inhibitors. Herein, we would like to present the discovery of VX-222.

\section{Sanjoy Kumar Das}

Dr. Sanjoy Kumar Das is working as an Associate Director at Angiochem since July 2013 where his research focus is to develop therapeutics for the treatment of neuro-oncology and lysosomal storage diseases. Prior to Angiochem, he was working at Vertex Pharmaceuticals as a Research Fellow where he was leading one of the chemistry teams to identify small molecule therapeutics for the treatment of inflammatory bowel disease (IBD). Before that, Dr. Das was leading the Hepatitis C medicinal chemistry group where his team discovered several clinical candidates, such as VX222 and VX-916. He also worked as an Associate Professor at the Université du Québec à Montréal (UQAM) for a short period of time in the beginning of 2004.

Dr. Das has received his $\mathrm{PhD}$ degree at Indian Institute of Chemical Technology, India. He worked with Professor Pierre Sinay at Ecole Normale Superieure, Paris, France (1996-1998) and Professor Rene Roy at Ottawa University, Ottawa, Canada (1998-2000) as a post-doctoral fellow before he joined Biochem Pharma as a research scientist in August, 2000. He has published over 45 papers and patents in the fields of organic chemistry and drug discovery. 


\section{Cross-institutional Drug Discovery Models: The OICR Experience}

\section{Rima Al-awar, Ontario Institute for Cancer Research (OICR)}

The Ontario Institute for Cancer Research (OICR) is an innovative translational research organization dedicated to research on the prevention, early detection, diagnosis and treatment of cancer. The mission of the Drug Discovery Program at OICR is to efficiently translate cancer-related academic discoveries into novel oncology therapies that will have a significant impact on the cancer patient population. The Drug Discovery group has successfully forged collaborations and partnerships that leverage the strengths and expertise within the program and across the academic community. The various resources and expertise available in the program will be presented along with examples of such successful collaborations.

\section{Rima Al-awar}

Dr. Al-awar earned a $\mathrm{PhD}$ in synthetic organic chemistry from North Carolina State University working on Lycopodium Alkaloids and did a postdoctoral fellowship focused on natural products synthesis at the University of North Carolina at Chapel Hill prior to joining Eli Lilly and Company in 1995. While at Lilly, she was an active medicinal chemist in the oncology area working in multidisciplinary teams on the antimicrotubule agent Cryptophycin and later on several kinase focused efforts. In 2002, while at Lilly, Dr. Al-awar took on administrative responsibilities as Head in Discovery Chemistry Research and Technologies and later as Head, Route Selection, in Chemical Product Research and Development prior to joining the Ontario Institute for Cancer Research as Director of the Drug Discovery Program in July 2008.

\section{Undruggable Means Undone}

Michael Serrano-Wu, Ph.D., Director of Medicinal Chemistry, Therapeutics Projects Group, Broad Institute

Human genetics has dramatically enhanced our ability to identify biological targets of compelling interest. Some of these well-credentialed targets, however, have earned the "undruggable" label because we as a community have yet to enable a pharmacological strategy towards target modulation.

MCL1 is one of such "undruggable" targets, and is among the most frequently amplified genes in cancer. MCL1 is highly expressed in many tumor types, and high expression levels of MCL1 contribute to tumor development and resistance to chemotherapy. Recently we discovered that the top biomarker for MCL1 dependency is the low expression levels of $\mathrm{BCL}-\mathrm{xL}$, and high expression levels of BCL-xL predicted resistance to MCL1 repressors. Despite overwhelming genetic and functional evidence implicating MCL1 as an attractive therapeutic target, effective therapeutic inhibitors have been difficult to develop.

Here, we describe our multi-prong strategy towards discovering selective MCL1 inhibitors. By leveraging a comprehensive toolbox of directbinding and cellular assays, we have developed complementary molecular approaches to targeting diverse aspects of MCL1 biology. A snapshot of our ongoing discovery efforts will be presented.

\section{Michael Serrano-Wu}

Mike Serrano-Wu joined the Broad Institute in 2012, and is the Director of Medicinal Chemistry for the Therapeutics Projects Group (TPG).

Serrano- $\mathrm{Wu}$ joins a team that aspires to transform the process of drug discovery and the treatment of human disease by developing and applying novel drug discovery technologies; targeting novel classes of protein targets, generally viewed as "undruggable"; targeting novel mechanisms emerging from studies of human biology; and working in an open, collaborative environment to establish proof of concept.

Serrano-Wu brings 15 years of drug discovery and development experience to the Broad. Previously at Bristol-Myers Squibb, he was part of an industry-leading group that discovered the firstin-class HCV NS5A inhibitor daclatasvir, now in late-stage clinical testing and showing promise for highly effective interferon-free therapy. Later at the Novartis Institutes for Biomedical Research, Serrano-Wu co-led a team that identified the first-inclass DGAT1 inhibitor pradigastat, currently in latestage clinical testing, followed by a secondgeneration compound that also achieved successful human proof of concept. Serrano-Wu also led a group tasked with drug discovery "beyond MW 500", which included the investigation of chemically-modified biotherapeutics.

Serrano-Wu received his A.B. and Ph.D. in chemistry from Harvard University. 


\section{The Discovery and Characterization of Orally Bioavailable Inhibitors of PTP 1B}

W. Cameron Black, Ph.D., Kaneq Pharma Inc.

Purpose: To identify orally active inhibitors of PTP$1 \mathrm{~B}$ for use in the treatment of Type 2 diabetes.

Methods: PTP-1B is a protein tyrosine phosphatase that dephosphorylates the insulin receptor, making it a negative regulator of insulin action. Inhibition of PTP-1B is an attractive method for increasing insulin sensitivity in the treatment of Type 2 diabetes. Small molecule inhibitors of this enzyme have typically employed doubly charged phosphonates as a pharmacophore, leading to compounds with low membrane-permeability and low oral absorption. In order to identify orally bioavailable inhibitors of PTP-1B, we sought to reduce the molecular weight of prototype inhibitors to enhance membrane permeability, taking advantage of available crystallographic data.

Results: Two promising series of inhibitors were identified, the thioether-linked phenyldifluorophosphonates, and the naphthalenedifluorophosphonates. SAR studies led to moderately potent inhibitors in each series which were found to have oral bioavailability of $5-15 \%$ in preclinical species. These inhibitors were orally active in standard rodent models of diabetes. The SAR, pharmacology and physical properties of these inhibitors will be discussed.

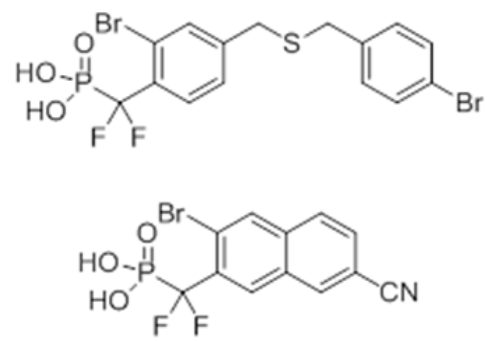

Conclusion: Sacrificing intrinsic potency to achieve oral bioavailability successfully led to a PTP-1B inhibitor, MF-598, which was active in three rodent models of diabetes. The lack of efficacy in the PTP-
1B knockout mouse demonstrated that the efficacy was due to on-target PTP-1B inhibition.

\section{Cameron Black}

Dr. Black holds a Ph.D. in chemistry from Harvard University and has 18 years of drug discovery experience with Merck Frosst. During this time he worked in the areas of inflammation, neurodegenerative disease, osteoporosis, metabolic disease and virology. Dr. Black was responsible for the identification of seven development candidates at Merck Frosst. Notably, he led teams responsible for the development of Previcox ${ }^{\circledR}$ and odanacatib, a cathepsin $\mathrm{K}$ inhibitor currently in Phase III development. He served as Director of Chemistry and Site Scientific Operations Lead at Merck Frosst and was responsible for resource allocation on all scientific programs. Dr. Black went on to co-found Kaneq Pharma in 2010 and Kanyr Pharma in 2013 to develop drugs targeting metabolic disease and cancer, respectively. He is the author of more than 50 scientific publications and is an inventor on 25 issued patents.

\section{Session Chair:}

\section{Arshad Siddiqui, PhD., President \& CEO, Paraza Pharma}

Dr. Siddiqui received a $\mathrm{PhD}$ degree in chemistry from the University of Waterloo, Ontario, Canada. $\mathrm{He}$ has twenty four years of experience in biotechnology and pharmaceutical companies including Shire-Biochem Pharma (Montreal, Canada), Neogenesis Pharmaceuticals (Cambridge, MA), Schering-Plough (Cambridge, MA) and Merck (Cambridge, MA). Dr. Siddiqui is a co-recipient of the Prix-Galien (Canada, 1996) for the discovery of Epivir, anti-HIV drug. An accomplished drug discovery scientist with over 70 research articles and 70 patents, he has founded Paraza Pharma Inc. in 2011. Currently he is President and CEO of Paraza Pharma Inc. 


\section{Wednesday, June 11 \\ SESSION 2:}

\section{Latest Topics in Bioequivalence}

\section{Expectations and Challenges in Small Molecules Regulated Bioanalysis}

Adrien Musuku, Ph.D., Director, Biopharmaceutics, Pharmascience

Study samples concentration data submitted to a regulatory agency as part of new or abbreviated new drug applications are expected to meet regulatory agency requirements in terms of quality, accuracy, precision and reproducibility as defined in published guidance documents on the conduct of bioanalytical method validation and samples analysis. The mainly followed guidances are those published by regulatory agencies such as the European Medicines Agency (EMEA) and the USFDA. The ultimate goal is to ensure that the reported analyte concentration is an accurate reflection of the sample concentration at the time of collection, is reliable and can be reproduced upon repeat analysis using the validated assay. Without confidence in the assay it would be impossible to make informed decision related to bioavailability determination or concentration response relationship. The most reliable bioanalytical data is usually generated using a bioanalytical method that has been validated and applied according to the principles of good laboratory practices (GLP). This presentation will highlight the challenges that can be encountered during the development; the validation and implementation of small molecule assays and the steps that can be taken to eliminate these challenges and provide regulatory agencies with data that meet their quality and reliability requirements and avoid common analytical findings throughout the review process.

\section{Adrien Musuku}

Adrien Musuku, Ph.D., Director Biopharmaceutics at Pharmascience, Montreal, Canada: Over 18 years of experience in management of Analytical and Bioanalytical Laboratory operations as well as clinical research including phase 1 development studies, drug-drug interaction and bioequivalence. He joined Pharmascience in 2010 and is a Director in charge of the bioanalytical development as well as the pharmacokinetic and clinical operations.

Prior to joining Pharmascience, he served as a Bioanalytical Director, Innovative Sector at Anapharm/Pharmanet in Quebec, Canada. Earlier, he was a Director, Laboratory Operations and Research and Development Director at Cantest in Vancouver, British Columbia, Canada.

He has a Doctor of Science degree in chemistry from the University of Antwerp in Belgium.

\section{NEW EMA Guidance for BE of Modified Release Products: Upcoming Challenges}

Fethi Trabelsi, PhD., Vice-President, Scientific Affairs, Biopharma Services Inc.

This presentation will provide an overview of the draft EMA guidance for the development of modified release dosage forms which was initially released for comments in March 2013 but still circulating for comments. The main objective of this guidance is to present guidelines and recommendations for the development of new entities as well as generics products having very specific modified release characteristics. Modified release product include products such as oral extended or delayed release, multi-phasic release, Intramuscular-subcutaneous depots and Transdermal drug delivery systems formulations. In this presentation, the focus will be on the EMA pharmacokinetics - safety requirements for generic products with some highlights of similarities and differences when compared to other requirements from major agencies such as Health Canada and US FDA. The scientific discussion will help understanding the rationale behind the EMA requirements and the potential impact on the Bioequivalence assessment. 


\section{Fethi Trabelsi}

Dr. Fethi Trabelsi is currently VP of the Scientific Affairs at Bio pharma Services Inc, a Canadian CRO located in Toronto. $\mathrm{He}$ is heading the biopharmaceutics, Medical writing and the bioanalytical teams, overseeing Study designs, Pharmacokinetics/Pharmacodynamics, Medical reporting, Statititcs/Data management of Phase 1 Clinical Trials. Studies include Bioequivalence/Bioavailability for generics and New entities drug development such as First in-Human, SAD-MAD, Drug-Drug-Interaction, QT prolongation and early clinical development for Biosimilars. Dr Trabelsi is also leading the bioanalytical Team in charge of method development and validation as well as sample analysis of small molecules. Dr. Trabelsi has around 15 years of experience in the Industry where he took increased responsibility and leadership roles. $\mathrm{He}$ holds a Masters and a $\mathrm{PhD}$ degree from the University of Montreal in Exercise Physiology and completed his post-doctoral training in the Pharmacology-Physiology field at the Faculty of Pharmacy at the University of Montreal. Before joining the Pharmaceutical Industry, Dr. Trabelsi has been lecturing Physiology courses at the University of Montreal for more than 8 years. He has published numerous scientific papers in peer-review journals and has presented in many National and International scientific conferences.

Dr. Trabelsi is a member of many scientific associations including the American Society of Clinical Pharmacology and Therapeutics (ASCPT) and the Generic Pharmaceutical Association (GPhA) in the US. He is also constantly interacting with many key Scientific and Regulatory experts at FDA, Health Canada and some EU agencies where he has attended many specific Pre-IND meeting with the regulators.

\section{Second Entry Respiratory Product Equivalence Requirements: A Comparison Between Jurisdictions}

Manon Bélisle, M.Sc., Director Biopharmaceutics and Clinical Development, Teva Canada Limited

Demonstration of equivalence for generic/second entry Orally Inhaled Products (OIPs) using in-vitro and in-vivo data is complex and scientifically challenging.
Device technology, patient handling device, formulation composition, active pharmaceutical ingredient characteristics, intended patient populations are all factors to be considered and which contribute to the complexity of generic/second entry development of OIPs.

This presentation will compare the current and proposed regulatory approaches in Canada, the United States and Europe for demonstration of therapeutic equivalence between two OIPs for use in the treatment of asthma and chronic obstructive pulmonary disease.

\section{Manon Bélisle}

Manon Bélisle is the Director of the Biopharmaceutics and Clinical Development Department at Teva Canada, a pharmaceutical company located in Toronto. In this role, she provides scientific leadership and managerial direction to the team responsible for all activities related to bioequivalence, clinical and toxicology studies. This work leads to generic drug approvals in Canada. Furthermore her team at Teva is also responsible of all onsite monitoring for studies conducted in Canada by Teva Global. She also held a similar position as Associate Director in Biopharmaceutics with ratiopharm Canada, before the company was acquired by Teva.

In the past, Manon has held positions of Senior Pharmacokinetic Scientist and Clinical Manager at a global CRO located near Montreal. She was responsible for developing protocol synopsis and clinical development plans for over 400 bioequivalence studies for pharmaceutical products from various therapeutic areas.

Manon is a graduate of the Universite de Montréal and Université du Québec in Montreal. She obtained a Bachelors of Science, Biochemistry and a Masters of Science, Physiology. During 5 years after obtaining her degrees she acted as a researcher for the University of Montreal in fundamental research for different areas including molecular biology, neurophysiology and metabolism.

Manon practices mind and body fitness incorporating meditation and physical activities in her life. In her spare time, Manon also loves to spend time in the outdoors appreciating the nature while skiing, canoeing and kayaking. As well, Manon is deeply interested in conscious living, appreciative inquiry and their application in professional and personal spheres. 


\section{What is TPD Working On?}

Eric D. Ormsby, Manager, Office of Science, Therapeutic Products Program, Health Canada

This talk will summarise the different issues which the Therapeutics Products Program (TPD) has or is currently working on. The first issue will be a quick review of the new guidance called Biopharmaceutics Classification System Based Biowaiver. This final guidance was posted on our web-site in mid May for immediate implementation. Summary of the proposed applications, requirements and comparisons with EMA and US FDA approaches will be given.

The second issue will be our interest in Bioequivalence Standards for highly variable drug products. This issue was brought to our advisory committee in December of 2013 and they recommended a need be shown that the current criteria are too onerous on industry. The CGPA has since provided some examples which will facilitate discussion at the next advisory committee meeting on June 20, 2014. A review of the options for widening the bioequivalence interval for these products will also be given at this meeting. Hopefully some direction from the committee will be given.
The final issue will be an overview of the impact of a recent court decision which interpreted "medicinal ingredient" differently than how TPD used the definition. This new interpretation affects many aspects of our work but most importantly on labelling and the "Identical Medicinal Ingredient Policy” for generics.

\section{Eric Ormsby}

Eric has worked for Health Canada for 33 years, almost entirely in some form of what is now called the Therapeutic Products Directorate (TPD). The TPD is responsible for pre-market assessment of pharmaceuticals and medical devices. Eric has been involved in bioequivalence issues since 1986 when Canada first began to develop a regulatory framework for bioequivalence.

Eric obtained a BSc. in Genetics and Statistics from the University of Guelph and a MSc. in Biostatistics also from Guelph. Currently he is manager of the Office of Science in the Bureau of Policy, Science and International Programs of TPD. This Office has the responsibility of managing $\mathrm{TPD}=\mathrm{s}$ access to external scientific advice, managing the reconsideration process and the development of science based regulations, policies and guidelines. 


\section{Wednesday, June 11}

\section{Plenary Lecture 1}

\section{Potential Applications of Human Stem Cells in Therapeutics}

Mickie Bhatia, Senior Scientist and Director of the McMaster Stem Cell and Cancer Research Institute (SCC-RI) McMaster University, Hamilton, ON

New advancements in human stem cell biology and related technologies have allowed visions of novel therapeutic approaches ranging from cell replacement therapies to genetic correction of human disease. One of the most impactful discoveries includes the demonstration of reprogramming cell fate by harvesting mature cells from adult patients, and altering the state to that of a stem cell with pluripotent capacity, called induced pluripotent stem cells or iPSCs. Generation of iPSC alleviates some ethical and immunological concerns regarding the source of pluripotent stem cells (PSCs) and in vivo rejection, respectively. Unfortunately, techniques to differentiate these iPSCs remain equally difficult and have similar limitations to pluripotent human embryonic stem cells already experienced years before. The next generation of reprogramming without transitioning through pluripotent state, termed direct conversion, will be discussed and placed into context with the use of human PSCs to accelerate the identification of chemical compounds leading to drug development for similarly targeted diseases, where cell transplantation may not be required.

\begin{abstract}
Mickie Bhatia
Dr. Bhatia's program seeks to understand the molecular mechanisms that govern somatic and pluripotent human stem cell development through; a) characterization of molecular pathways and genomic targets that regulate hematopoietic and pluripotent stem cells differentiation, and b) the use of novel in vivo models for cellular/tissue regeneration through xeno-transplantation. As a recognized leader in human stem cell biology and applications, Dr. Bhatia's work has been published in several major journals over the years, and his program continues to focus on two central areas; 1) developing abundant sources of human hematopoietic progenitors, and 2) using human stem cells to develop treatments to eliminate tumor reoccurrence. Dr. Bhatia also serves as a scientific consultant to government and industry, and to medical companies interested in stem cell-based technologies, and sits on numerous editorial and scientific advisory boards.
\end{abstract}




\title{
Wednesday, June 11
}

\author{
SESSION 3:
}

\section{Successful Candidate Selection from Druggability to Regulatory Submission}

\section{Physicochemical and Biopharmaceutical Characterization of Development Candidates - What do we Need to Know?}

\section{Elizabeth Vadas, InSciTech}

Even before identifying a development candidate, drug discovery groups must look beyond the biological properties of potential leads from which a development candidate maybe selected. Specificity for the target, potency of the molecule, metabolism and lack of overt toxicity are clearly critical attributes of a putative drug molecule but these attributes alone will not make it a viable development candidate. Regardless of the route of administration, there are some physiochemical characteristics that are key to the viability of the molecule as a drug candidate. Solubility, solid state and solution stability are a few of these attributes to which the discovery group must pay attention during lead optimization and when selecting a development candidate, although key information is not limited to these properties.

The information one needs to have regarding a development candidate falls into three different categories:

a. Critical knowledge, information one must have,

b. Important knowledge, information that needs to be generated early in development but not absolutely critical for initiating development activities

c. Information that is nice to have, not critical for initial development but may become important later.

The presentation will outline the different types of information, critical, important and nice to have that play a role in candidate selection and their impact in defining an early development strategy.

Elizabeth B. Vadas, Ph.D.

Elizabeth B. Vadas received her Ph.D. in Physical Chemistry from McGill University in Montreal. She obtained her undergraduate degree in colloid and surface chemistry in Budapest, Hungary. Following postdoctoral training in the Department of Biochemistry at McGill she joined Merck Frosst, the Canadian subsidiary of Merck \& Co. in 1980 where she has spent 22 years in the department of Pharmaceutical Research and Development. Over the years she has been involved in the early characterization and formulation development of many new chemical entities discovered at the Merck Frosst Centre for Therapeutic Research while taking on increasing management responsibilities.

Prior to her retirement from Merck, Dr. Vadas was Executive Director of Pharmaceutical Research and Development at Merck Frosst. Most notable of her scientific leadership accomplishments was the establishment of very close collaboration with the discovery groups in basic research resulting in the rapid characterization and selection of viable development candidates. Her most significant technical accomplishments at Merck Frosst were the product development efforts of the department under her leadership, which lead to worldwide regulatory approval of several products, among them SINGULAIR ${ }^{\circledR}$, Merck's oral asthma therapy, and VIOXX ${ }^{\circledR}$ and ARCOXIA ${ }^{\mathrm{TM}}$, two Cox-2 inhibitors for the treatment of pain, osteoarthritis and rheumatoid arthritis.

In mid-2002 Dr. Vadas decided to take early retirement to establish her own consulting company InSciTech Inc., a company providing integrated solutions for scientific and technical problems faced by life science companies in the compound selection, physicochemical and biopharmaceutical characterization of development candidates and product development areas.

Dr. Vadas' main scientific interests are in the area of pharmaceutics, particularly in solid state chemistry and physics, drug excipient interactions and identifying appropriate means of delivering molecules of low solubility. She has been an Adjunct Professor of Pharmaceutics between 1995 and 2005 at the Faculty of Pharmacy, University of Montreal. She also has lectured and published widely and has received numerous scientific and management awards. She has been elected a Fellow 
of both CSPS and AAPS.

\section{Trainee Presentation}

\section{Synthesis, Preparation, and Characterization of Polymeric Nanocapsules of Nifuroxazide Analogues}

(See Poster Abstract \# 60)

Michael Jefferson Amorim de Oliveira, University of Sao Paulo

\section{Playing your Cards Hard and Early, But Smart: Advances in Increasing the Odds of Successful Development of Drug Candidates}

Andrey Peresypkin, PhD, Director, Materials Discovery and Characterization, Vertex Pharmaceuticals, Inc., Boston, MA

The discovery/development interface often has a point of "no return" where a company is "all-in" with regard to advancing a drug candidate into clinical and pharmaceutical development. "Hot" compounds can advance rapidly into early tox studies and straight into FIH. Typically, the major "formulation goal" is to come up with a material (form) and a formulation to maximize exposures (for tox \& pharmacology studies); however, downstream CMC developability (or in other words the ability of the drug to avail itself to efficient and scalable manufacture, be chemically and physically stable, and be suitably bioavailable through formulation for clinical trials and ultimately for commercial sale) remains often overlooked as programs gamble on being able to make it work later. To ensure sound decision making, an early and clear quantitative assessment of the ability to progress a candidate to POC and beyond is desirable. Achieving this aim has its challenges including, among other things, limited availability of the drug substance, aggressive use of formulation vehicles, institutional priorities, and limited tools to predict material properties. We report here on some recent advances in small scale development and characterization tools and materials / formulations / permeability enhancing technologies. These advances may not only allow for a more rapid progression into non-clinical and early clinical studies, but also may lend themselves to careful quantitative analysis of the feasibility on turning candidates into suitable dosage forms for later stage clinical trials and commercial use.
Andrey Peresypkin

Andrey Peresypkin obtained his $\mathrm{PhD}$ in Organic Chemistry from the lab of Fred Menger at Emory University (Atlanta/ GA, USA). During his graduate school tenure, his research interests focused on gaining deeper understanding of the aggregation behavior of novel amphiphilic molecules including gemini surfactants, amphiphilic dendrimers, and others. Following his $\mathrm{PhD}$ in 2001, he joined Pharmaceutical Chemistry group at Merck and Co. (Rahway/ NJ, USA) specializing in the area of early pre-formulation (discovery support, animal/toxicology formulations, solid form, clinical formulations support, etc.) and eventually taking over the leadership of that group. In 2007, Andrey joined Vertex Pharmaceuticals, Inc. (Boston/ MA, USA) where he currently works as Director in MDC (Materials Discovery and Characterization) department managing groups in Boston (MA), San Diego (CA) and Oxford (United Kingdom). The group is responsible for addressing various preformulation type activities from early Discovery (PK/tox/pharmacology formulation development, solid form discovery, solid-state characterization and methods, etc.) all the way to registration \& NDA.

\section{Believing is Seeing: Breakthrough Thinking in Pharmaceutics \& Drug Delivery}

Riccardo Panicucci, Global Head of Chemical and Pharmaceutical Profiling (CPP) at Novartis

The pharmaceutical industry is facing a shift away from traditional drug therapies and is seeking new opportunities in incorporating new technology into drug development and delivery. In order to stay relevant for our next generation of patients, we need to embrace new ways of using technology to improve and personalize patient care. This presentation will discuss the challenges and opportunities in drug formulation and delivery. Specifically this presentation will touch on the challenges of predicting human pharmacokinetics using animal bioavailability data and explore the promising developments in using innovative technology to improve drug targeting and drug delivery, such as oral deliveries of peptides and proteins and unique combinations of drugs, devices, and technologies. Finally, the presentation will also 
touch on new business models to best move forward, specifically advocating for greater collaboration between academia, government, and pharmaceutical industry.

\section{Riccardo Panicucci}

Global Head of Chemical and Pharmaceutical Profiling (CPP) at Novartis from 2004. Global responsibility for Research support and early Pharmaceutical Development of small molecule therapeutics. Additional responsibilities include developing novel drug delivery technologies for small molecules and large molecules including siRNA. Currently holds the position of Adjunct Professor at Massachusetts College of Pharmacy.

Previously held positions include the Director of Formulation Development at Vertex Pharmaceuticals, Director of Research and Development at Symbollon Pharmaceuticals and Senior Scientist at Biogen. He started his career at Bausch \& Lomb in the pre-formulation lab and was promoted to Manager of Process Development.

His education includes two post doctoral fellowships at University of California at Santa Barbara and the Ontario Cancer Institute. He received his Ph.D. in Physical Organic Chemistry from the University of Toronto.

\section{Session Chair:}

\section{Sophie-Dorothee Clas, Ph.D., Consultant,} PharmaSolv Consulting, Montreal, QC,

Dr. Clas established PharmaSolv Consulting after working for 23 years in drug development at Merck \& Co., Inc. Prior to launching PharmaSolv
Consulting, Dr. Clas worked for 3 years in the Basic Pharmaceutical Sciences group in West Point, PA providing physico-chemical characterization and pre-clinical tox formulation support to the Discovery teams in the areas of infectious diseases, neurosciences and endocrine. She contributed to the approval of several compounds which are currently in Phase I clinical studies. She also led the Global Prodrug Expert Team at Merck with a mandate to promote using prodrugs in Discovery. Between 2008 and moving to Merck in West Point, PA, Dr. Clas was responsible for guiding the selection of the drug substance phase and preclinical tox formulations in infectious disease at Merck Frosst Canada; which led to the development of one compound currently in Phase II. She served as global scientific advisor, providing guidance and mentoring on phase and formulations. Prior to this, Dr. Clas led the Preformulation group at Merck Frosst Canada for 17 years until restructuring in 2008, providing materials characterization support to the development of new drug candidates in the anti-inflammatory disease area, from Phase I to filing. During this time, her team contributed to the development of for example, Singulair $^{\circledR}, \quad$ Vioxx $^{\circledR}, \quad$ Arcoxia $^{\circledR}, \quad$ laropiprant (Tredaptive $^{\circledR}$ ), veterinary Cox-2 inhibitors (e.g. Equioxx ${ }^{\circledR}$ ), as well as compounds that are presently in Phase II to III studies, such as odanacatib, a Cat K inhibitor for osteoporosis in Phase III. Dr. Clas is also adjunct professor in the Faculty of Pharmacy at the University of Montreal. She has been an invited lecturer at many symposia and conferences and has contributed to 76 oral and poster presentations. She is co-inventor of 11 patents and co-author of 43 refereed publications and 15 preprints and abstracts. 


\section{Wednesday, June 11 \\ SESSION 4:}

\section{Regulatory Considerations and Analysis of Subsequent Entry Biologics}

\section{Canada's Approach to Subsequent Entry Biologics (SEBs)}

Agnes Klein, MD, DPH, Director, Centre for the Evaluation of Radiopharmaceuticals and Biotherapeutic Products, Biologics and Genetic Therapies Directorate, Health Canada

Subsequent Entry Biologics (biosimilars in other jurisdictions) are a new class of biological products. Despite the difference in nomenclature for Canada, the overall approach to regulations is similar in all those countries where SEBs are becoming available on the market.

This presentation will discuss the approach to the regulation of SEBs in Canada, the rationale for the approach and show how there are similarities in approach overall. The presentation will also discuss the approach Canada has taken to two critical points, namely extrapolation of indications and interchangeability or substitutability of these products.

\section{Agnes V. Klein}

Agnes Klein is currently the Director, Centre for the Evaluation of Radiopharmaceuticals and Biotherapeutic Products in the Biologics and Genetic Therapies Directorate.

Dr. Klein received her medical degree from the University of Toronto. She trained in Endocrinology, Medical Biochemistry and Public and Community Health. She joined Health Canada and the Drugs Directorate in late 1974 and has occupied many and varied scientific and management positions within Health Canada and its regulatory arms, both on the pharmaceuticals and the biologics side. Amongst relevant accomplishments, Dr. Klein represented Health Canada on NCBHR and NCEHR and chaired the Committee on Clinical Trials of the Council.

Dr. Klein's special interests include the appropriate design of clinical trials and the various and complex ethical issues attendant to the design and conduct of clinical trials and other studies in human subjects. Dr. Klein just completed serving as a member of Health Canada's Research Ethics Board; she was a member of the board for about 10 years and continues to be consulted on many related issues.

Dr. Klein was an active participant in the CIOMS document on Pharmacogenetics and Pharmacoeconomics, as well as member of the expert working group for ICH E-15 and ICH E-16. More recently, Dr. Klein has been active in the area of biosimilars (Subsequent Entry Biologics [SEBs]) and has made many presentations on various aspects of this subject.

Dr. Klein is an active member of several medical and scientific organizations nationally and internationally

Dr. Klein has received the Outstanding Service Award from DIA as described below:

The DIA Outstanding Service Award is given to recognize those individuals who consistently, through their volunteer efforts, have made contributions to the DIA mission and vision over the past several years. These individuals have exceeded expectations in their volunteer activities with DIA.

In 2012, Dr. Klein received the Queen's Diamond Jubilee Medal for Services rendered to the Health of Canadians.

\section{Regulatory Considerations for Biosimilars in the United States}

Sue Lim, M.D., Senior Staff Fellow, U.S. Food and Drug Administration, Center for Drug Evaluation and Research, Office of New Drugs

In March 2010, the Affordable Care Act was signed into law, which gave FDA the authority and responsibility to regulate a newly defined class of medical products known as biosimilar biological products. This Act established an abbreviated 
licensure pathway for biological product $\mathrm{s}$ that are demonstrated to be biosimilar to or interchangeable with an FDA-licensed reference product.

This presentation will provide an overview of FDA's recommended approach to the development of proposed biosimilar products as discussed in three draft guidances on biosimilar development published in February 2012. Specific development concepts will also be discussed.

\section{Sue Lim}

Dr. Sue Lim is a Senior Staff Fellow with the U.S. FDA, Office of New Drugs, Therapeutic Biologics and Biosimilars Team. In this role, she provides review and oversight of therapeutic biologics and biosimilar products, as well as regulatory policy development for biosimilars. Dr. Lim was previously a medical officer reviewer with the FDA's Division of Anti-Infective Products. Prior to the FDA, Dr. Lim served as an Infectious Diseases physician and Associate Hospital Epidemiologist at the University Health Network in Toronto, Canada. She received her M.D. from the University of Toronto where she also completed her residency in Internal Medicine and subspecialty training in Infectious Diseases and served as a Lecturer in the Faculty of Medicine. She has a Master's degree in Health Policy, Management and Evaluation from the University of Toronto.

\section{Analytical Tools for Characterizing Biologics}

\section{Steven A. Berkowitz, Ph.D., Consultant}

During the course of developing a biopharmaceutical an enormous amount of information is collected by the manufacturer to help demonstrate and convince regulators of their ability to develop a stable and consistent drug product that offers sufficient therapeutic value that merits its approval as a commercial drug. This aggregate of information falls into a number to different categories that include biological, biochemical, biophysical and clinical information. In this presentation the analytical tools that support the acquisition of both biochemical and biophysical data used in developing and commercializing these drugs will be discussed.

In biochemically characterizing biopharmaceuticals we are predominately concerned with the task of studying the primary structure of these complex molecules. In this area mass spectrophotometry (MS) reigns supreme. Using both intact MS and MS in combination with peptide mapping a high level of biochemical characterization information can be acquired in order to assess the relatively high level of micro-heterogeneity commonly encountered in working with this class of drug molecules. However, a key weak link in this characterization work is the frequent lack of information on the potential impact that a detected chemical change can have on the higher-order structure (HOS) of these protein drugs. This situation is especially true when these chemical changes are outside the spatial regions of these molecules that are concerned with their biological activity.

In biophysically characterizing biopharmaceuticals, no one tools stands out. In fact, our ability to acquire biophysical information on the HOS of these drug molecules is significantly lacking in detail. Much of the information we acquire in this areas is derived from a collection of low resolution tools. Nevertheless, in recent years there has been significant activity aimed at trying to improve this situation.

Overall, the picture concerning our ability to analytical characterize biopharmaceutical is not perfect, but at the same time it must be realized it has greatly improved since the first modern biopharmaceutical was approved. In this talk we will thus also take a look at some of the ongoing work aimed at closing the gaps in our capability to better biophysically and biochemically characterize biopharmaceuticals in order to make better informed decisions in their development and evaluations in their approval as commercial drugs.

\section{Steven A. Berkowitz}

Dr. Berkowitz received his B.S. degree in Biology from Fairleigh Dickinson University and his Ph.D. degree in Biochemistry from New York University. He then spent several years as a post-doctoral fellow at Yale University and the NIH. After his postdoctoral work Dr. Berkowitz held various positions at Celanese Research Company, J.T. Baker, and Lederle Laboratories before taking a position at Biogen Idec where he has spent his last twenty year working in the areas of purification, analytical and formulation development before retiring at the end of 2013.

Dr. Berkowitz's technical areas of expertise are concentrated in the separation and physical sciences associated with the characterization of a wide range of biopolymers and synthetic polymers. Much of Dr. Berkowitz's work has centered on assessing the 
physicochemical properties, micro-heterogeneity, aggregation properties and higher-order structure of biopharmaceuticals using a range of biochemical and biophysical techniques that has predominately involved the use of light scattering, analytical ultracentrifugation, chromatography, electrophoresis, and various forms of spectroscopy.

\section{Assessment of Biosimilarity and Interchangeability}

Laszlo Endrenyi, Professor Emeritus, Pharmacology and Biostatistics, University of Toronto

Biological drugs are much more complicated than drugs of small molecules. Their large size, complexity of structure, manufacture and analysis, the related sensitivities, the high possibility of immunogenicity are some of the contributors to the complications. Therefore it is much more difficult to compare the similarity of biological than of smallmolecule drug products. For one, follow-on products cannot reproduce exactly the brand-name, reference drug. The assessment of sufficient similarity cannot rely on a straightforward contrast but must utilize a complicated procedure which involves several factors and requires a judgment based on all available evidence. An additional complication in comparing biological products is that their similarity and interchangeability must be assessed separately. The risk of comparing the test (T) and reference (R) products should not be significantly higher than the risk of repeatedly evaluating the reference drug. Therefore the similarity of the $T$ vs. R. contrast should be comparable to the reproducibility of the $\mathrm{R}$ vs. $\mathrm{R}$ contrast. The statement can be statistically quantified. It expresses the expectation on the required closeness of similarity. The approach can be extended to the quantitative determination of interchangeability by applying it to relevant crossover designs. The comparison of the risks permits the evaluation of the probability for allowable interchangeability.

\section{Laszlo Endrenyi}

Laszlo Endrenyi is Professor Emeritus of pharmacology and biostatistics in the University of Toronto. He has served the university in various positions including on its Governing Council and as Associate Dean of Graduate Studies. He sat on the Board of Directors of the American Statistical Association and the Canadian Society for Pharmaceutical Scientists; he was a president of the latter. Externally, he has served on grant review committees and editorial boards of research journals including the Amer. J. Physiol, J. Pharmacokin. Pharmacodyn., J. Pharm. Pharmaceut. Sci., Biosimilars, and J. Pharm. Sci. He has received several recognitions, including an honorary doctorate from the Semmelweis University of Medicine. He is a Fellow of the Canadian Society for Pharmaceutical Sciences and of the American Association of Pharmaceutical Scientists.

Dr. Endrenyi published a book on Kinetic Data Analysis and over 180 research papers. Several of these established principles for the design and analysis of enzyme and pharmacokinetic investigations. More recently, he extensively developed principles and applications for the evaluation of bioavailability, bioequivalence and biosimilarity. His studies were instrumental in the adoption of some regulations and the withdrawals of others. He has consulted with the Food and Drug Administration and Health Canada and served on their advisory committees. He has consulted also with industry in the areas of pharmacokinetics, biostatistics, the design and evaluation of experiments, clinical trials, and the analysis of bioavailability, bioequivalence and biosimilarity studies. 


\section{Wednesday, June 11 \\ SESSION 5:}

\section{Nanomedicine}

\section{Strategies to Overcome Challenges Facing Nanomedicines}

Christine Allen, Ph.D., Professor and Interim Associate Dean Graduate Education, Leslie Dan Faculty of Pharmacy, University of Toronto

Enhanced tumour accumulation of nanomedicines relative to conventional chemotherapeutic agents has long been a hallmark of nanoparticle-based therapy. However, significant challenges hamper the clinical translation and viability of nanomedicines; namely, variability in the enhanced permeability and retention (EPR) effect, poor tumour penetration of nanotechnologies and limited bioavailability of drug. In this presentation, several of these key barriers will be discussed with strategies our laboratory is employing to overcome these challenges introduced. In addition, particular emphasis will be placed on the integration of imaging and image-based methods to aid in the rational design and advancement of nanomedicines.

\section{Christine Allen}

Christine Allen is a Professor and currently serving as the interim Associate Dean Graduate Education in the Leslie Dan Faculty of Pharmacy at the University of Toronto. Her research is focused on the rational design and development of new materials and technologies for the delivery of drugs and contrast agents (Lab Website: http://phm.utoronto.ca/ allen/). Allen completed her doctoral research in the Department of Chemistry at McGill University and post-doctoral research in the Department of Advanced Therapeutics at the B.C. Cancer Agency. She joined University of Toronto in 2002, from Celator Pharmaceuticals Inc. (Vancouver, B.C.) where she had worked as a scientist and Assistant Director of materials research. She has 90 publications, numerous patent applications, and nine book chapters on both lipid and polymer-based delivery systems. She has served on several peer review panels for granting agencies including CIHR (2004, 2005-2009, 2011), NCIC $(2005,2006)$ and NIH $(2006,2008,2011$ - 2017). She was awarded a CIHR-Rx\&D Career Award (2004-2009) for her research on the design and development of technologies for cancer treatment. She has also received the Association of Faculties of Pharmacy of Canada/AstraZeneca New Investigator Research Award, the Canadian Society Pharmaceutical Science/GlaxoSmithKline Early Career Award, an Innovation Award from the Ontario Research Commercialization Program and the Jorge Heller Controlled Release Society (CRS) Best Paper Award in 2011. In addition, she was recently awarded an endowed GlaxoSmithKline Chair in Pharmaceutics and Drug Delivery (effective July 2014). She is an Associate Editor for the ACS journal Molecular Pharmaceutics and involved in numerous scientific societies including as an elected member on the board of directors of CRS.

\section{Trainee Presentation}

NMR and Fluorescence Studies of pH-sensitive Liposomes Based on a Molecular Switch (See Poster Abstract \#22)

Warren Viricel, Université de Montréal

\section{Phenylboronic Acid Modified Mucoadhesive Nanoparticles Facilitate Weekly Treatment of Dry Eye Syndrome}

Frank Gu, Canada Research Chair and Associate Professor, Department of Chemical Engineering, University of Waterloo

Purpose: To develop mucoadhesive nanoparticle drug carriers that improve the precorneal retention of the drugs, and to evaluate their in vivo ocular irritancy response and efficacy in treating experimental dry eye by delivering Cyclosporine A (CycA).

Methods: The nanoparticles were formed using 
block copolymer poly(D,L-lactide)-b-dextran and surface functionalized with phenylboronic acid. The encapsulation and release properties of the nanoparticles were assessed using CycA. Experimental dry eye was induced in mice and treated with CycA loaded nanoparticles (weekly administration) and compared with the thrice daily administration of RESTASIS $\AA$. We assessed these mice using tear volume measurements, fluorescein staining, and histopathology. The nanoparticles, with or without CycA, were administered weekly to one eye of each rabbit, while the contralateral eye served as control. We examined the acute (1 week) and chronic (12 weeks) irritation responses using slitlamp bio-microscope and histopathology.

Results: The nanoparticles encapsulated up to 11.2 $\mathrm{wt} \%$ of CycA and sustained the release for 5 days. Histopathology demonstrated that administering CycA-loaded nanoparticles to dry eye-induced mice weekly eliminated inflammatory infiltrates and completely recovered the ocular surface. While thrice daily administration of RESTASIS ${ }^{\circledR}$ also cleared the inflammatory infiltrates, it showed much slower ocular surface recovery. Moreover, the weekly dosage of nanoparticles did not cause any irritation or inflammation throughout acute (1 week) and chronic (12 weeks) irritancy studies.

Conclusion: The mucoadhesive nanoparticle formulation significantly reduced administration frequency without compromising the therapeutic efficacy, while also improving the ocular surface recovery by eliminating irritation associated with frequent administration. The study provides promising results for the potential application of this formulation to dramatically improve therapeutic efficacy in treating anterior eye diseases.

\section{Frank Gu}

Prof. Frank $\mathrm{Gu}$ is a Canada Research Chair and Associate Professor in the Department of Chemical Engineering at the University of Waterloo. He has established an interdisciplinary research program combining functional polymers and polymer-metal oxide hybrid materials to solve problems in medicine, agriculture and environmental protection. Dr. Gu received his BSc from Trent University and $\mathrm{Ph} . \mathrm{D}$. from Queen's University, Canada, where he majored in chemical engineering and was awarded with Canada Graduate Scholarship from Canadian Natural Sciences and Engineering Research Council (NSERC). Following completion of his graduate program, he was awarded a NSERC Postdoctoral Fellowship to purse his research at Massachusetts
Institute of Technology and Harvard Medical School. In July 2008, Dr. Gu joined Department of Chemical Engineering at the University of Waterloo as an Assistant Professor. In 2012, he was awarded Canada Research Chair to advance his research in the development of targeted delivery systems using nanotechnology. His expertise in the development of functional nanoparticles for targeted delivery has generated over 100 scientific publications in peer reviewed journals and conference proceedings, as well as 15 US and World patent applications.

\section{Therapeutic Potentials of Carbohydrate Based Nanomedicine}

Ravin Narain, Associate Professor, Department of Chemical and Materials Engineering, University of Alberta, Edmonton, AB

Carbohydrates are known to play a significant role in many important cellular recognition processes, including cell growth regulation, differentiation, adhesion, cancer cell metastasis, cellular trafficking, inflammation by bacteria and viruses, and immune response. With recent progress in the field of carbohydrate science, polymer science and nanotechnology Carbohydrate based therapeutics have emerged as a remarkable and promising new class of therapeutics with huge potentials. In recent years, glycopolymers and glyconanoparticles have been synthesized with remarkable control on the architecture and compositions to exhibit nearly similar properties to naturally occurring glycoproteins and polysaccharides. Carbohydratebased drug and gene delivery systems have been developed, in which the carbohydrate receptors are used to direct drugs to specific organs or cells. This talk will highlight the recent developments in the design and fabrication of such carbohydrate based systems and their applications in biomedicine.

\section{Ravin Narain}

Ravin Narain, $\mathrm{PhD}$, is Associate Professor in the Department of Chemical and Materials Engineering at the University of Alberta. He has published over 100 articles in high impact, peer-reviewed journals and is the editor of two Wiley books entitled "Engineered Carbohydrate-Based Materials for Biomedical Applications" (2011) and "Chemistry of Bioconjugates" (2014). Dr. Narain's research examines nanomedicine and regenerative medicine, with an emphasis on developing new biomaterials 
for drug delivery, gene therapy and tissue engineering.

\section{Session Chair}

\section{Todd Hoare}

Todd Hoare is an Associate Professor in the Department of Chemical Engineering at McMaster University. Hoare specializes in engineering hydrogels and microgels with targeted "smart" properties and was the first to report glucoseresponsive microgels for diabetes treatment and externally-triggerable, electronics-free magneticallyactivated implantable drug delivery devices. Hoare's work has been profiled by Popular Science, Wired, and $B B C$ for its potential in solving clinical challenges through innovative biomaterials design. He has published over 45 papers, has one granted patent and eight pending patent applications, and won an NSERC Innovation Challenge award recognizing the novelty of his research. Hoare has received an Early Researcher Award and the John Charles Polanyi Prize in Chemistry in recognition of his accomplishments in his early career as a faculty member. He is also an Associate Editor of Chemical Engineering Journal for materials engineering and is a member of the editorial advisory board for Colloid and Polymer Science. 


\section{Thursday, June 12}

\section{Plenary Lecture 2}

\section{Current Paradigm of Cancer Nanomedicine and its Perspective - A Gap Between Experimental \& Clinical Worlds}

You Han Bae, Ph.D., Professor, Department of Pharmaceutics and Pharmaceutical Chemistry, College of Pharmacy, University of Utah, Salt Lake City, UT, USA

Countless nano-sized carriers (nanocarriers) of anticancer drugs have been designed in pursuit of improved efficacy and reduced side effects. Thus far, designs have been based on the following principles: long circulation with stealth surface, small in size (20-200 nm) for EPR effect, stability in the blood compartment, responsive properties to stimuli from external sources or intrinsic tumor microenvironment, and specific interactions with target cancer cells. For decades, this area of research has shown remarkable promise in curing or suppressing cancer growth in preclinical models. However, there is a surprising deficiency in translating these nanocarrier systems to clinical use. As part of an effort to understand this gap between preclinical and clinical outcomes this lecture reviews the results of clinical trials with nanocarrier formulations, either marketed or withdrawn technologies, and re-evaluates the individual design principles in clinical settings. An effort will also be made to compare pharmacokinetics, toxicity, and efficacy research on animal and human studies. Lastly, the possible causes for this barrier to successful translation are discussed. Emphasis is especially placed on intratumoral distribution due to the primacy of chemotherapeutic drugs reaching the target site. Consequently, multidrug resistance and tumor heterogeneity and dynamics are also significant factors for consideration. Although rodent cancer models are mandatory before clinical trials, their predictive power is desperately low (5$10 \%$ ). A new paradigm and more predictive preclinical models need to be established in therapeutic cancer nanomedicine to advance the future of nanocarrier design.

\section{You Han Bae}

Dr. You Han Bae is Professor of the Department of Pharmaceutics and Pharmaceutical Chemistry, University of Utah. He served University of Utah as a postdoctoral fellow and research assistant professor till 1994. He joined the Department of Materials Science and Engineering at Gwangju Institute of Science and Technology (Korea)-a graduate school-in 1994 as an associate professor with the first departmental chairmanship and promoted to a full Professor in 1998. After serving 7.5 years at GIST, he re-joined the University of Utah as a full professor in 2002.

His early research covered temperature-sensitive polymers and their applications for delivery, and functionalized polymers for biohybrid artificial pancreas. His research then shifted, for the last 15 years, to protein drug stabilization and controlled release, self-assembled super $\mathrm{pH}$-sensitive nanoparticulates for multidrug resistance in cancer and tumor heterogeneity, and novel polymeric vector design for the delivery of genetic materials. He has published over 250 peer-reviewed scientific papers, book chapters and U.S. Patents, which have been cited over 18,000 times ( $h$-index: 70) (Google Scholar, as of April 2014). His new research directions are: understanding of extravasation, EPR effect, intratumoral transport of nanoparticles; more predictable preclinical cancer models; specific nanoparticle absorption in the GI track; and nonviral gene therapy for metabolic diseases.

He has been elected as fellows of the American Institute of Medical and Biological Engineering, the American Association of Pharmaceutical Scientists, and the American Academy of Nanomedicine. He served Controlled Release Society (CRS) as a member of Board of Scientific Advisory (20002003), as a member, co-chair and chair of Young Investigator Award committee (1999-2007) and as a program co-chair for the $34^{\text {th }} \mathrm{CRS}$ annual meeting at Long Beach, CA (2007). He has been a co-chair of International Symposium on Recent Advances in Drug Delivery System (Salt Lake City, Utah) since 2006. He is an editorial board member of Pharmaceutical Research and Bioconjugate, and is also currently serving the Journal of Controlled Release (JCR) as an America Associate Editor as well as the Editor of The Concept Paper in JCR. 


\section{Thursday, June 12 \\ SESSION 6:}

\section{Going Beyond Oral Delivery ... Strategies to Enable Drugs to Reach New Targets}

\section{Seven-Day Microneedle Enhanced Transdermal Delivery: Bench to Bedside}

Audra Stinchcomb, PhD, RPh, School of Pharmacy, University of Maryland, Baltimore, MD USA \& AllTranz Inc., Lexington, KY, USA

Transdermal microneedle systems have become a very popular means of delivering skin impermeable drugs through the stratum corneum at therapeutic rates. Most of the previous research on microneedle systems has focused on optimization of the microneedle geometry. Our approach to microneedle-assisted delivery research has been in the following areas:

1. Investigation of prodrugs and salt forms with optimal physicochemical properties for drug flux after microneedle treatment

2. Investigation of viscosity and other formulation factors that influence drug flux after microneedle treatment

3. Investigation of micropore lifetime using transepidermal water loss (TEWL), impedance spectroscopy, and pharmacokinetic analysis

4. Investigation of micropore lifetime after treatment with COX inhibitors

5. Investigation of codrugs with optimal physicochemical properties for drug flux and micropore lifetime after microneedle treatment.

Highly water soluble salts have provided the best microneedle-assisted flux, as exemplified by our studies with the model compound naltrexone (NTX). The flux from a PEGylated naltrexone prodrug through microneedle-treated skin showed pronounced deviation from ideal behaviour. Accounting for changes in viscosity of the donor solution allowed rationalization of the data.

Microneedle (MN) application in hairless guinea pigs followed by TEWL and impedance spectroscopy monitoring of micropore lifetime showed that good correlation existed between the two methods of analysis. COX enzymes in the skin are known to be involved in inflammation, wound healing, and keratinocyte differentiation. Thus, the hypothesis that COX inhibition may increase micropore lifetime was evaluated in hairless guinea pigs and humans. Micropore lifetime was extended up to 7 days by using the nonspecific COX inhibitor diclofenac in combination with MN treatment in guinea pigs. A pilot human study was done for evaluation of the effects of diclofenac on micropore lifetime, utilizing impedance spectroscopy. A second human study for evaluation of the pharmacokinetics of NTX in combination with MN's and diclofenac was also completed. Finally, a codrug strategy was used to enhance flux and micropore lifetime after $\mathrm{MN}$ treatment.

We would like to acknowledge the National Institutes of Health for providing funding for this project (R01DA013425, R42DA32191 and R21DA31439).

\section{Audra L. Stinchcomb}

Dr. Stinchcomb is Professor of Pharmaceutical Sciences, School of Pharmacy, University of Maryland, Baltimore, and Chief Scientific Officer and Founder of AllTranz Inc, a transdermal specialty pharmaceutical company. She received her Bachelor's in Pharmacy from the University of Colorado in 1989, and a PhD in Pharmaceutics in 1995 from the University of Michigan. She completed a postdoctoral fellowship at UCSF, and then joined the faculty at the Albany College of Pharmacy for 4 years. She was a Professor at the University of Kentucky from 2001-11, and joined the faculty at UMB in November 2011. Research interests have included transdermal prodrugs, microneedle-enhanced delivery, and translational research models for public-private partnerships.

Dr. Stinchcomb's research program has revolved around the examination of the mechanisms and optimization of prodrug skin permeation and microneedle-enhanced skin permeation. This research has studied the influence of prodrug 
physicochemical properties on skin flux, distribution, and metabolism. The results of this prodrug knowledge have fueled the genesis of AllTranz, a transdermal specialty pharmaceutical company. Transdermal prodrugs are in development for treatment of cancer chemotherapy nausea and vomiting, alcoholism, drug addiction, and pain. Transdermal cannabinoid delivery has also been a major research focus, with new prodrugs developed to improve aqueous solubility, chemical stability, as well as skin permeation. The microneedle research focus has been on formulations for improved permeation rate and micropore lifetime, utilizing animal studies translated to human subject studies.

\section{Fabricated Nano and Microparticles for Non-oral Delivery}

Brian Farrer, Principal Scientist, Liquidia

By leveraging fabrication techniques from the semiconductor industry, Liquidia's PRINT® platform has the ability to rapidly design and manufacture precisely engineered particles of virtually any size, shape, or composition. Presentation will focus on the application of PRINT ${ }^{\circledR}$ to non-oral drug delivery. A description of the PRINT ${ }^{\circledR}$ fabrication process will be followed by a discussion of some of the benefits of the technology to non-oral drug delivery. A more in depth discussion of non-oral applications will include in vitro and in vivo results from preclinical studies using the PRINT ${ }^{\circledR}$ particles for ophthalmic or pulmonary delivery.

\section{Brian Farrer}

Brian Farrer joined Liquidia Technologies as a Principal Scientist in 2012 where he is working to develop platform non-oral formulations using the innovative particle fabrication process. Prior to this he spent 10 years in Merck's Basic Pharmaceutical Sciences group providing a wide variety of preclinical formulations and development candidate risk assessments. Brian received his $\mathrm{PhD}$ in inorganic chemistry from the University of North Carolina-Chapel Hill in 1999.

\section{Trainee Presentation}

RNAi-Based Nanomicrobicide for the Prevention of Male-to-Female Transmission of HIV-1 (See Poster Abstract \#35)

Sidi Yang, University of Manitoba

\section{Paradigm Shifts in Ocular Drug Delivery}

Heather Sheardown, Professor of Chemical Engineering, Faculty of Engineering. McMaster University, Hamilton ON

Traditional methods of delivery of drugs to the eye include eyedrops and more recently injection of therapies into the vitreous cavity of the eye. While the former is well accepted, the inability to target intraocular tissues and significant losses of the active agent make this a suboptimal method. As well, eye drop therapies are limited by patient compliance. Direct injection into the back of the eye, while effective, has a high incidence of complications and is therefore only useful for treatment of certain advanced conditions. It is clear that there is a real need for better methods of drug delivery. This talk will focus on novel methods of drug delivery to the eye that overcomes some of these issues and the challenges faced by each of these methods, including some discussion of the new materials that are being used to facilitate delivery. Mucoadhesive materials have the potential to increase the residence of drugs in the precorneal tear film, thereby decreasing the need for frequent drop instillation. However, turnover of the cells of the precorneal epithelium remains a limiting factor. Contact lens based drug delivery has shown promise in the lens wearing population but may not be appropriate for patients who do not currently wear lenses. Insertable scaffold materials can be placed in the back of the eye to facilitate long term drug release. However, removal, both at the end of the useful life of the device and in the event of complications will be discussed. In situ gelling materials show significant promise for maintaining therapeutic concentrations over a long time period. Targeted systems which use light as well as other methods to facilitate release are also underdevelopment. These methods will lead to a paradigm shift in ocular drug therapy. 


\section{Heather Sheardown}

Dr. Sheardown (PhD, Chemical Engineering and Biomedical Engineering, University of Toronto, BEng, Chemical Engineering, McMaster University) is a professor in the Department of Chemical Engineering at McMaster University. She joined the Faculty of Engineering at McMaster in 1998, and served as Associate Dean (Graduate Studies) from 2009-2013. She holds an adjunct appointment at the School of Optometry at the University of Waterloo.

Her research interests are focused primarily on the development of biomaterials and drug delivery systems for treating ocular disorders. Her previous studies have included work on the development of novel contact lens materials, artificial cornea and intraocular lens materials and on the development of novel methods of delivering drugs to the eye. She has published over 120 refereed articles, has been an invited speaker at many conferences, and has trained 10 PhDs.

She is the Scientific Director of the 20/20 NSERC Ophthalmic Materials Strategic Network and has led a number of other initiatives related to development of new materials in the eye. She was awarded the McMaster University Innovator of the Year award and has been recognized as the Hamilton Halton Engineer of the Year. She sits on a number of grant panels for NSERC and CIHR. She has served as a consultant to or worked with a number of companies in the field of ophthalmic materials and drug delivery as well as to Health Canada. She has served as associate editor and on the editorial boards of several journals.

\section{Designing and Engineering a Multipurpose Delivery Platform for Prevention and/or Treatment of Sexually Transmitted Infections}

Jill Steinbach, PhD, Department of Bioengineering, University of Louisville

Sexually transmitted infections (STIs) affect 340 million new people per year, and exert a significant impact on global health. Despite the crucial need to develop prophylactic agents and therapeutics, vaccines and antiviral agents have only been moderately successful in preventing STIs, including human immunodeficiency virus (HIV) and herpes simplex virus 2 (HSV-2), and curing them postinfection. Microbicides, in comparison, have the promising potential to prevent and treat STIs, while providing simultaneous female-controlled protection against a diversity of infections and unplanned pregnancy. The development of a safe, effective, durable, and multipurpose microbicide that is tailored to the intravaginal environment is an urgent need.

During my postdoctoral position, we had demonstrated efficacy in the design and application of PLGA (poly(lactic-co-glycolic acid)) nanoparticles (NPs) that encapsulate short interfering RNA (siRNA) molecules, designed to interfere with cellular targets involved in HSV-2 infection. We showed that topical, vaginal delivery of these NPs increased survival after lethal genital HSV-2 infection in mice from $\sim 9$ days (in untreated animals) to 28 days in PLGA NP treated animals. To enhance NP penetration in a variety of cell lines, we hypothesized that cell binding and internalization could be improved by modifying the NP surface with cell penetrating peptides. Using two different surface modification strategies to attach these peptides to the NPs, we demonstrated that cell binding and internalization are significantly enhanced in vitro. In parallel, we explored the mechanism of cell uptake and intracellular fate of these vehicles to aid in the rational design of NPs for optimal gene delivery.

Building upon these delivery abilities in my new faculty position, we seek to apply the concepts of mucosal protection against virus infection and the delivery impediments posed by mucus, to the design of gene and drug delivery vehicles tailored to the intravaginal environment. Currently we are developing a multipurpose virus "trap" microbicide platform that has the ability to: 1) physicochemically immobilize and debilitate HSV/HIV before it can bind to host cells, 2) integrate two different delivery modalities to provide sustained-release of encapsulated antiviral and biological agents in parallel, and 3) promote the $\mathrm{pH}$-sensitive release of carriers from our multipurpose delivery platform. We believe this hybrid of delivery carriers, materials, and preventative/therapeutic agents should enable versatile, safe, and efficacious protection/treatment options against STIs.

\section{Jill M. Steinbach}

Dr. Steinbach is a new Assistant Professor of Bioengineering in the Center for Predictive Medicine at the University of Louisville, with an associate appointment in the Department of Pharmacology and Toxicology. She received her B.S. in Materials Science Engineering from the University of Illinois at Urbana-Champaign, and 
completed her M.S.E. at Arizona State University while working part-time in industry. In the hopes of using her engineering background for more direct medical applications, she transitioned into Bioengineering and conducted her graduate work in Dr. Michael Caplan's lab at Arizona State University. Following her Ph.D., she had the opportunity to do her postdoctoral fellowship in Dr. W. Mark Saltzman's laboratory in the Department of Biomedical Engineering at Yale, working on the design of gene and drug delivery vehicles targeting host and virus genes in a murine model against HSV-2 infection.

Dr. Steinbach's long-term goals are to create drug and gene delivery vehicles that provide more efficacious prophylactics and treatments for sexually transmitted infections (STIs), including acute and chronic (latent) infections. In addition to developing better vehicles that specifically target viruses and host cells, significant advancements can be made to rationally design delivery platforms targeted to the unique microenvironments where infection, latency, and reactivation occur. Gene and drug delivery vehicles, especially those suitable for delivery to the peripheral and central nervous systems (PNS/CNS) - sites of HSV latency - are still in the nascent stages of development. Similarly, the development of next-generation microbicides, offers burgeoning opportunities to create biomaterial combinations that can adapt to the acidic and mucosal intravaginal environment and provide multipurpose (contraceptive and viral) prevention modalities.

Dr. Steinbach has served as a mentor in the training of 20 high school, undergraduate, and graduate students, and one current postdoctoral associate. In the past 9.5 years (graduate and postdoctoral), her work has been supported by the NIH F32 postdoctoral fellowship, NSF IGERT, and Yale Proteomic/Genomics Institute. She was (honored to be) chosen as the NSF IGERT fellow to represent the ASU IGERT program during her graduate work. Her additional awards include: the
Dept. of Bioengineering Leadership Award (Akay Award at ASU), and fellowships: (1) NSF and National Academy of Sciences, (2) NSF for Biocomplexity and Systems Biology, to share her research in seminars abroad in Turkey. She has also received conference travel grants and has served as both the Vice President (inaugural) and President of the Connecticut Controlled Release Society (CRS) Student Chapter.

\section{Session Chair}

\section{Karine Khougaz, Pharmascience}

Karine Khougaz is an accomplished pharmaceutical scientist and project leader with over 15 years in the pharmaceutical industry. After completing her PhD studies at McGill University studying block copolymer micelle self-assembly, she pursued an NSERC sponsored Postdoctoral fellowship at Merck in Montreal in the characterization of solid dispersions and drug solubilization in surfactant systems. Karine then joined the preformulation group at Merck responsible for the physicochemical characterization of numerous compounds from discovery to commercialization. In addition, she was involved in the phase and form selection of drugs for toxicology and clinical studies. For a five year period, she successfully managed a crossfunctional team responsible for the drug substance and drug product for a portfolio of compounds from preclinical assessment to Phase IIB clinical studies. Karine subsequently joined Pharmascience as a Project Leader managing the portfolio of innovative products for potential in-licensing. Karine is currently working in Regulatory Affairs at Pharmascience, involved in the screening and due diligence of potential in-licensed products and in the preparation of New Drug Submissions for the approval of new products into the Canadian market. 


\section{Thursday, June 12 \\ SESSION 7:}

\section{New Trends in Mass Spectrometry for Drug Discovery \& Development}

\begin{abstract}
Mass Spectrometry in the Pharmaceutical Industry: Past, Present and Future
\end{abstract}

Lucinda Cohen, Ph.D., Director, NJ Discovery Bioanalytical Group, Merck Research Laboratories

[Abstract not available]

\section{Lucinda Cohen}

Lucinda (Hittle) Cohen received her Ph.D. in Analytical Chemistry in 1994 from the University of Pittsburgh under the direction of Professor David M. Hercules. She then worked with Professor Franz Hillenkamp at the University of Muenster, Germany as an Alexander von Humboldt postdoctoral fellow. She began work in 1996 with Parke-Davis Pharmaceutical Research in the Pharmaceutical Delivery Systems, Analytical Research division in Morris Plains, NJ. From 1999-2007, Lucinda worked in the Pharmacokinetics, Dynamics \& Metabolism department within Parke-Davis, Ann Arbor, MI which became Pfizer Global Research and Development. Lucinda joined Merck in 2007 and currently is Director of the PPDM New Jersey Discovery Bioanalytics Group, which provides quantitative in vivo and in vitro analytical support for projects in Kenilworth and Rahway. She has served as an adjunct faculty member for Stevens Institute of Technology, Hoboken, NJ. Her research interests include mass spectrometry, bioanalysis, and sample preparation approaches to increase the quality and speed of high throughput analysis in a drug discovery environment.

\section{Current Advancements in Large Molecule Bioanalysis by High Resolution Mass Spectrometry (HRMS)}

Fabio Garofolo, Vice President Bioanalytical Services, Algorithme Pharma, Laval, Quebec

Ligand binding assays (LBA) are a common approach to large molecule bioanalysis including the PK determinations of biologics and the measurement of biomarker concentrations in biological samples. However, during the last few years LC-MS has evolved as a reliable technique for the accurate quantitation of therapeutic proteins and large peptides in biological fluids by offering a valid alternate to LBA. Indeed, large molecule quantification using LC-MS is becoming a leading technique in the pharmaceutical and biotechnology industry. LC-MS instead of LBA in quantification of biologics has many advantage such as no needs for high affinity reagent; uniform approach; large linear range; higher selectivity and well accepted by Regulatory Agencies. It is commonly performed by using tryptic digestion, then purifying and detecting one or more small signature peptides by triple quadrupole instruments. However, sometimes, triple quadrupoles are not specific enough for the signature peptides quantification coming from large molecules due to high background noise, unspecific fragmentation, unacceptable variability, lack of selectivity to eliminate isobaric interferences, need for long cleanup and chromatography. In the past, High Resolution Mass Spectrometry (HRMS) had limitations in terms of sensitivity, linear dynamic range and speed, to fulfill the need for reliable quantification in bioanalysis. However, the new generation of HRMS is able to overcome these limitations and it could become "the ultimate tool for large molecule bioanalysis." With increasing demand in biotherapeutics/biologics programs in pharmaceutical industry, HRMS opens new 
opportunities for quantification of peptides and proteins in biological samples during pharmacokinetic studies. The wide scan range on HRMS instruments is advantageous for intact peptide or protein quantification and these instruments are able to increase the selectivity while maintaining sensitivity. This presentation will focus on describing the main challenges and providing practical solutions on proteins and peptides quantification in a regulated environment with and without tryptic digestion by HRMS. Multiple concrete case studies of increasing complexity will be presented and advantages of HRMS versus triple quadrupoles demonstrated for Large Molecules bioanalysis.

\section{Fabio Garofolo}

Dr. Fabio Garofolo has been working in the pharmaceutical and analytical fields for more than 22 years. Capitalizing on an extensive occupational background within his specialization field, he has also been involved to help scientific non-profit organizations (AAPS, ASMS, CFABS, CVG, GBC, GCC and NBC) with the mission to promote discussion on the pharmaceutical practices.

Dr. Garofolo has progressed throughout in his career in both the pharma/biotech and CRO industries and benefited from successfully overcoming the prevalent challenges in drug development. Since 2005 he has held the position of Vice-President Bioanalytical Services at Algorithme Pharma. Dr. Garofolo has over 130 publications \& presentations in international conferences. He developed around 300 innovative analytical methods. He designed and invented 3 innovative bioanalytical approaches and is the recipient of the following Lilly awards: Achievement (2001); Global (2002); Emmerson (2003). His current interests include analysis of large molecules by LCMS, HRMS, DBS, and Emerging Technologies.

\section{Trainee Presentation}

Optimization of a PAMPA-BBB Model and Subsequent Validation by a Consolidated LCMS/MS Method

\section{(See Poster Abstract \#6)}

Florian Bernard, Université de Montréal

\section{Chemical Proteomics: Identification of Drug Targets and Mechanisms}

Juergen Kast, The Biomedical Research Centre, University of British Columbia

In its broadest sense, chemical proteomics is defined as the study of the interactions between chemical compounds and the protein content of a cell or tissue. In pharmaceutical research, where chemical proteomics finds its most prominent applications, it is mostly used to investigate the effect of drugs, or drug metabolites, on cells. Two distinct areas of application have emerged: one focuses on the identification of the proteins that bind the drug, the other on the protein changes the drug induces. For each, several approaches exist: for direct drug interactions, they differ in whether the drug itself or a drug analogue is used, whether the analogue is designed to bind its target covalently or non-covalently, and in how the drugprotein complex is isolated. Similarly, a variety of approaches are available for drug-induced proteome changes, dependent upon the anticipated type of change and the known or postulated mechanism of action. In my presentation, I will give an overview of the field, discuss each of these approaches, and illustrate their potential by providing examples of their use.

\section{Juergen Kast}

Dr. Juergen Kast obtained his $\mathrm{PhD}$ in Analytical Chemistry from the University of Konstanz, Germany in 1998. Following a post-doctoral research stay at the European Molecular Biology Laboratory in Heidelberg, Germany, he joined the University of British Columbia in Vancouver in 2001. He currently holds an appointment as Professor at The Biomedical Research Centre and in the Department of Chemistry. Between 2009 and 2011, he served as Scientific Director of the British Columbia Proteomics Network, before taking a 2-year leave of absence from academia to join industry. Between 2011 and 2013, he worked as Director of Proteomic Discovery, then Head of Therapeutic Target Discovery, at Oxford Biotherapeutics Ltd in the UK. In 2013, Dr. Kast returned to UBC to continue his research, in which he develops and applies proteomics methods for the analysis of disease mechanisms, particularly those involving blood platelets. 


\section{Ion Mobility in Pharmaceutical Applications}

Colin S. Creaser, Centre for Analytical Science, Department of Chemistry, Loughborough University, Loughborough, UK

Ion mobility (IM) spectrometry separates gas phase ions in ways that are complementary to separation according to mass-to-charge ratio by mass spectrometry. In drift tube IM, ions are separated on the basis of collision cross section (size and shape) as they pass along a drift tube under the influence of a static low field gradient or travelling wave. In field asymmetric waveform ion mobility spectrometry (FAIMS), also known as differential mobility spectrometry, ions are separated by differences in their high field and low field mobility as they pass between two electrodes. In both techniques, separation is determined by collisions between the ions and a buffer gas, usually nitrogen, air or helium. This presentation will briefly describe these ion mobility techniques and provide an overview of their application to pharmaceutical measurements including metabolite and peptide determination in biofluids, metabolite profiling and the monitoring of potentially genotoxic impurities.

\section{Colin Creaser}

Colin Creaser was educated in Uganda and the UK, receiving his $\mathrm{BSc}$ and $\mathrm{PhD}$ degrees from the University of Kent, followed by postdoctoral research at the University of California at Santa
Barbara. He has held appointments as Senior Scientific Officer at AEA Harwell, Senior Lecturer in Analytical Chemistry at the University of East Anglia and Professor of Analytical Chemistry at Nottingham Trent University, UK. In 2007, Colin moved to his present appointment as Professor of Analytical Chemistry and Head of the Centre for Analytical Science in the Department of Chemistry at Loughborough University. He is currently ViceChair and Chair-elect of the British Mass Spectrometry Society.

\section{Session Chair}

\section{Lekha Sleno, Université du Québec à Montréal}

Associate professor in the chemistry department at UQAM (Université du Québec à Montréal) (since 2008) where her research interests focus on reactive metabolites covalently binding to proteins and developing new metabolomics-based methods using liquid chromatography coupled to mass spectrometry (LC-MS).

Originally from Montreal, did her BSc (2002) in Biochemistry at Concordia University, followed by a $\mathrm{PhD}$ (2006) at Dalhousie (Chemistry), focused on bioanalytical mass spectrometry. She then went on to the University of Geneva in Switzerland for a first post-doc in pharmaceutical mass spectrometry, working on reactive drug metabolites, followed by a 2nd post-doc at University of Toronto in proteomics. 


\section{Thursday, June 12 \\ CSPS Lifetime Achievement Award Lecture}

\section{Sense and Sensibility (?) in Science}

Laszlo Endrenyi, University of Toronto

Laszlo Endrenyi is Professor Emeritus of pharmacology and biostatistics in the University of Toronto. He has served the university in various positions including on its Governing Council and as Associate Dean of Graduate Studies. He sat on the Board of Directors of the American Statistical Association and the Canadian Society for Pharmaceutical Scientists; he was a president of the latter. Externally, he has served on grant review committees and editorial boards of research journals including the Amer. J. Physiol, J. Pharmacokin. Pharmacodyn., J. Pharm. Pharmaceut. Sci., Biosimilars, and J. Pharm. Sci. He has received several recognitions, including an honorary doctorate from the Semmelweis University of Medicine. He is a Fellow of the Canadian Society for Pharmaceutical Sciences and of the American Association of Pharmaceutical Scientists.

Dr. Endrenyi published a book on Kinetic Data Analysis and over 180 research papers. Several of these established principles for the design and analysis of enzyme and pharmacokinetic investigations. More recently, he extensively developed principles and applications for the evaluation of bioavailability, bioequivalence and biosimilarity. His studies were instrumental in the adoption of some regulations and the withdrawals of others. He has consulted with the Food and Drug Administration and Health Canada and served on their advisory committees. He has consulted also with industry in the areas of pharmacokinetics, biostatistics, the design and evaluation of experiments, clinical trials, and the analysis of bioavailability, bioequivalence and biosimilarity studies. 


\section{Thursday, June 12 \\ CSPS Award of Leadership in Canadian Pharmaceutical Sciences Lecture}

\section{A Bright Future for Pharmaceutical Sciences in Canada}

Rav Kumar, Vice-President - R\&D Operations/ Business Development, GSK Canada

Dr. Rav Kumar leads GlaxoSmithKline's Canadian R\&D/Business Development organization which includes Clinical Research, Regulatory Affairs, External R\&D Alliances and New Business Development as well as GSK's Established Products portfolio in Canada.

Rav is a pharmacy graduate with a $\mathrm{PhD}$ in pharmaceutical sciences (from Bath University, UK) involving research into controlled delivery of drugs such as insulin. He has more than 25 years of global drug development experience having worked for start-up and multinational pharmaceutical companies in the United Kingdom, France and North America. Rav has been very engaged in the Canadian Pharmaceutical R\&D "Ecosystem" over the past decade including serving as President of the Canadian Society for Pharmaceutical Sciences (CSPS), Vice-Chair of Regulatory and Medical/Clinical Committees at Rx\&D and Chair of several DIA Canada conferences.

Rav was instrumental in bringing together academia, industry and government for the "Canadian Clinical Trials Summit" held in Ottawa, to develop an action plan for improving Canada's competitiveness in this area. This resulted in the following report: To Your Health and Prosperity - An Action Plan to Help Attract More Clinical Trials to Canada as well as the recent creation of the Canadian Clinical Trials Co-ordinating Centre (CCTCC) - a collaborative effort of CIHR, Rx\&D, and ACAHO/CHA.

Dr Kumar has served on various boards and councils including: Member of steering committee for CHIR/SPOR (Strategy for Patient Oriented Research); Lesley Dan Faculty of Pharmacy Advancement Board; Board of CQDM (Quebec Consortium for Drug Discovery); University of Toronto Mississauga Campus Council; Board of Hillfield Strathallan College, Hamilton.

Rav has guided GSK into important public private partnerships and collaborations for drug discovery with CDRD, CQDM and MaRS Innovation as well as the GSK $\$ 50 \mathrm{M}$ Life Sciences Innovation Fund. These have resulted in numerous investments in the Canadian Life Sciences ecosystem.

He also serves as President of SAPNA - a nonprofit organization dedicated to improving the health and Health awareness of South Asian Canadians. He has given talks at numerous conferences, events and academic institutions on topics such as Building Trust in the Pharmaceutical Industry, New Models for Drug Discovery and Development and Careers in life sciences. 


\section{Thursday, June 12 \\ SESSION 8:}

\section{Biomarkers in Pharmacotherapy and Drug Development}

\section{Oxytocin as a Biomarker of Stress Disorders \& Relational Difficulties: Therapeutic Implications}

Kathleen Hegadoren, University of Alberta

Despite the hype in the mass media as the "love peptide" or "liquid trust", there is little empirical evidence that intra-nasal oxytocin (IN OT) has efficacy as a treatment for relational difficulties. Open-field trials of IN OT in those with autism, schizophrenia, social anxiety disorder have not shown robust results. Past interpersonal violence experiences (childhood maltreatment and adult violence) can have multiple long term physical and mental health impacts for women, including a sense of betrayal and pervasive distrust. Two gender specific theories relate stress, mood, anxiety symptoms and relational difficulties in women to oxytocin's interactions with the HPA axis. A preclinical observational cohort design is underway to identify relationships among measures of trust and plasma levels of OT in a large cohort of premenopausal women (total $\mathrm{N}=400 ; \mathrm{N}=137$ to date), grouped by history of interpersonal violence or no such history ( $\mathrm{N}=69 \& 68)$, while controlling for factors that may influence OT levels or domains of trust. Results from preliminary data analysis for the 2 groups of women include: multiple differences in demographic and lifestyle parameters, significant differences in specific domains of trust and a trend towards differences in OT levels by trust domain. Salivary cortisol levels also differed at all time points. There was an interaction between violence experiences and oral BCP use in predicting OT levels. Our preliminary data support the importance of considering multiple biological and psychosocial parameters when investigating OT as an intervention for women with mood and anxiety symptoms and relational difficulties related to past interpersonal violence experiences.

\section{Kathy Hegadoren}

Dr. Kathy Hegadoren is a Professor in the Faculty of Nursing at the University of Alberta and a Canada Research Chair in Stress Disorders in Women. Her clinical background in mental health and basic science training has helped her take an integrated science approach to understanding biological and psychosocial mechanisms involved in the increased vulnerability in women to mood and anxiety disorders. Her specific areas of research involve the impact of interpersonal violence on women's health and perinatal mental health.

\section{Trainee Presentation}

Correlation between Arachidonic Acid Metabolites and Renin-angiotensin System peptides in Adjuvant Arthritis Rat (See Poster Abstract \#42)

Ali Aghazadeh-Habashi, University of Alberta

\section{Biomarkers in Osteoarthritis and Rheumatoid Arthritis}

Roman Krawetz, PhD, Grace Glaum Professor in Arthritis Research, Assistant Professor, Departments of Surgery and Cell Biology \& Anatomy, McCaig Institute for Bone and Joint Health, University of Calgary

Osteoarthritis (OA) is the most common chronic joint disease for which there is no known cure. In its 2011 report, the Arthritis Alliance of Canada reported that $\mathrm{OA}$ affects 1 in 8 Canadians (more than 400,000 Albertans); within Alberta, productivity losses related to OA had a negative economic impact of $\$ 2.75$ billion in 2010 alone. By 2040 more than 1 in 4 Canadians will have OA and $30 \%$ of the labour force will have difficulty working as a result. The objective of our work in this area is to develop a method for categorizing normal individuals as well 
as patients with (OA), and to distinguish these populations from patients with rheumatoid arthritis (RA) based on a panel of inflammatory cytokines expressed in serum samples. Two panels of inflammatory proteins were used as training sets in the construction of two separate artificial neural networks (ANNs). The first training set consisted of all proteins (38 in total) and the second consisted of only the significantly different protein expressed (12 in total) between at least two patient groups. Both ANNs obtained high levels of sensitivity and specificity, with the first and second ANN each diagnosing $100 \%$ of test set patients correctly. These results were then verified by re-investigating the entire data set using a decision tree algorithm. We have shown that ANNs can be used for the accurate differentiation between serum samples of patients with OA, a diagnosed RA patient comparator cohort, and normal/control cohort. Using neural network and systems biology approaches to manage large datasets derived from high throughout proteomics approaches should be further explored and considered for diagnosing diseases with complex pathologies such as OA.

\section{Roman Krawetz}

Dr. Krawetz completed his PhD with Dr. Greg Kelly from The University of Western Ontario, Canada examining how murine stem cells make the decision to differentiate into cells/tissues that make up the extra-embryonic structures that sustain the early embryo.

In 2006, Dr. Krawetz was recruited to Dr. Derrick Rancourt's laboratory at the $\mathrm{U}$ of $\mathrm{C}$ to pursue postdoctoral training. His work demonstrated the usefulness of mouse and human embryonic stem cells for regenerative medicine approaches in bone and cartilage tissue engineering, and he developed the first method to scale up the generation of human embryonic stem cells. In 2010, Dr. Krawetz was recruited to the University of Calgary's McCaig Institute as an independent investigator has shifted his research focus to synovial stem cell biology to further understand their role in the onset and pathogenesis of Osteoarthritis and cartilage regeneration.

His long term goals are: (1) Translate his observations regarding synovial stem cell biology into novel therapeutics that would reduce the need for more expensive or invasive procedures in patients suspected of having early OA, (2) define the factor(s) responsible for stem cell loss of function, block their effect and reverse/slow progression of
OA, and (3) develop novel diagnostic approaches to effectively identify OA patients at the early stage of the disease when they might be more receptive to treatment.

Furthermore, it is Dr. Krawetz's hope that the development of reliable and cost effective biomarker assays for osteoarthritis will lead to facilitated OA drug development. Since many pharmaceutical companies have withdrawn from the development of OA drugs, due in part to the difficulties in selecting, stratifying and following patient outcomes, the identification and development of new biomarkers could potentially re-invigorate pharmaceutical investment in OA and improve the quality of life of patients suffering from this disease.

\section{Arachidonic Acid Metabolism Profile as Biomarker of Cardiac Function in Inflammatory Conditions: Arthritis}

Fakhreddin Jamali, Professor, Faculty of Pharmacy \& Pharmaceutical Sciences, University of Alberta Fakhreddin Jamali, Ali Aghazadeh-Habashi, Waheed Asghar

Biomarkers can improve pharmacotherapy and accelerate drug development. Biomarkers that reflect outcomes that are difficult to follow or those that emerge before catastrophic events are highly desirable; markers such as blood pressure and cholesterol have been in use for many decades. Inflammatory conditions and many drugs that are used to treat them are associated with cardiovascular (CV) implications. This is while there is no clear measure to facilitate prediction of emergence of $\mathrm{CV}$ implications. This, places the caregiver and the patient at a cross-road: 'Deprive the patient from the treatment or risk the CV side effects'. Similarly, a screening tool to facilitate development of CV safe antiinflammatory drugs is timely. We have previously reported that experimental arthritis causes an imbalance of various cardioprotective and cardiotoxic elements in the heart. Antiinflammatory drugs that restore this balance are also shown to have favorable $\mathrm{CV}$ safety profile when used by patients with arthritis. We have now found that there is a correlation between the changes in the heart and arachidonic acid metabolites in plasma, hence, the latters can be used as biomarkers to detect the emergence of cardiotoxicity by both the disease and the drugs used for treatment. Arachidonic acid metabolites patterns may serve as useful biomarkers 
in pharmacotherapy of patients with inflammatory conditions and drug development.

\section{Fakhreddin Jamali}

Dr. Jamali (Doctor of Pharmacy, University of Tehran; MSc, pharmaceutics and $\mathrm{PhD}$, pharmacokinetics, University of British Columbia) is a professor at the Faculty of Pharmacy and Pharm. Sci., University of Alberta. He joined the faculty at the University of Alberta in 1981.

His research interests include effect of pathophysiological changes on the action and disposition of drugs, stereochemical aspects of drugs action and disposition, basic and clinical pharmacology of anti-rheumatic, analgesic and cardiovascular drugs, and toxicology of nonsteroidal antiinfammatory drugs. $\mathrm{He}$ has published 220 refereed articles (H-Score, 41) and has been an invited speaker at many conferences, and has trained 35 PhDs.

$\mathrm{He}$ is a principal investigator with the Centre of Excellence for Gastrointestinal Inflammation and Immunity Research and also a member of the Canadian Arthritis Network (Networks of Centres of Excellence, served as a Theme Leader).

For his academic achievements and research, he has been appointed is a Fellow of the Canadian Soc. Pharm. Sci (CSPS), the American Assoc. Pharm. Sci., and the American College of Clin. Pharmacology. He has received the Killam Professorship, McKeen Cattel Memorial Award of the American College of Clin. Pharmacol., the McCalla Professorship of the University of Alberta, the McNeil Award of Assoc Canadian Faculties of Pharm., CSPS/DuPond Resaerch Leadership Award of the. For his service to the public he has been honored with the Alberta Centennial Medal and the Alberta Phamacy Centennial Award of Distinction.

Dr. Jamali has served as a consultant and/or a member of the board of directors of many pharmaceutical houses. He has been a member of the Health Canada's TPP Expert Advisory Committee on Bioavailabilty and Bioequivalence, and the Expert Advisory Panel on Nonsteroidal Antiimflammatory Drugs. He is the founding president of CSPS and editor of J. Pharm. \& Pharm. Sci. (www.cspsCanada). He has served as associate editor or in editorial board of several journals. He teaches pharmacokinetics and is involved in pharmacy curriculum development. 


\section{Thursday, June 12}

SESSION 9:

\section{Application of Quality by Design (QbD) and Lifecycle Management to Analytical Procedure}

\section{SPONSORED BY: COLORCON}

\section{Implementation of Analytical Quality by Design Concepts at Pfizer}

Loren Wrisley, Senior Director, Analytical Research and Development, Pfizer, Groton, CT

At a high level, the talk will attempt to illustrate why $\mathrm{QbD}$ is important to analytical chemists and we will flesh out some concepts such as the ATP (analytical target profile). We will look into the basic analytical $\mathrm{QbD}$ process and tools currently used at Pfizer, supported by case studies. These will include a glimpse of workflows that incorporate $\mathrm{QbD}$ elements such as risk assessments and designed experiments, a total error approach to assess method performance, the design of meaningful method controls, etc. The discussion will naturally lead to some thoughts around possible future direction within the industry.

\section{Loren Wrisley}

I began my career with Ayerst Laboratories in Rouses Point, NY in 1983 in the Separations group of Analytical Research and Services. Our R\&D group had a close association with QA at this site, which has certainly played a part in my desire to provide practical, robust solutions to analytical challenges. My career has focused on pharmaceutical/analytical development on projects spanning pre-"proof of concept" through launch and commercialization. My groups and I have supported a variety of customers including Chemical Development, Toxicology, Formulations Development, Clinical, Environmental, etc., primarily with separation and dissolution method development, validation and assays. I am particularly passionate about experimental design
(QbD) and separation optimization via modeling.

\section{Trainee Presentation}

Greyscale Inkjet Printing of Personalised Levothyroxine Doses and Optical Dose Verification

(See Poster Abstract \#72)

Mustafa Alomari, UCL School of Pharmacy

USP Stimuli Article on Lifecycle Management of Analytical Procedures

Elisabeth Kovacs, Chief Scientific Officer Chemistry and Analytical Sciences, Apotex

In the Lifecycle Management of Analytical Procedures: Method Development, Procedure Performance Qualification, and Procedure Performance Verification, stimuli article posted on July 26, 2013 on the USP website, the Expert panel proposes that the traditional approaches to validation, transfer, and verification should be integrated into the analytical procedure lifecycle process rather than be viewed as separate entities. ICH Guidelines; Q8, 9, and 10 provide a life cycle model that has been developed for pharmaceutical products. Same concepts can also be applied to Analytical Procedures. Identifying the output of the analytical procedure as the reportable result provides a target for development and helps to ensure the procedure is developed towards predetermined performance requirements that are directly linked to the quality of the data, ATP (Analytical Target Profile). The presentation will illustrate how following $\mathrm{QbD}$ principles during 
method development will lead to better understanding how variables will impact on the quality of the data and how employing science based quality risk management, control strategy, and routine monitoring should ensure that data generated by the analytical procedures will meet the predefined quality standard stated by the ATP.

\section{Elisabeth Kovacs}

Elisabeth Kovacs works at Apotex as Chief Scientific Officer Chemistry and Analytical Sciences. She has been at Apotex for over 30 years in roles of increasing responsibility involved in all aspects of Generic Product Development (CMC focus), formulation development ( $\mathrm{QbD}$, dissolution development, BCS, Biowaivers,) establishment of API and product specifications, analytical method development and validation. In her role she has extensive interaction with regulatory agencies TPD, FDA, EU AU, mostly via developing strategies for submission reports, responses to deficiency questions and follow up. Elisabeth is member of several USP Expert Panels and Project teams, GPhA Science and Advisory Committee, GPhA USP Task Force, AAPS; IVIVR Focus Group Steering Committee, and the CSPS board of directors
Implementation of QbD (Quality by Design) to Analytical Procedures - FDA Perspective

\section{Sharmista Chatterjee, ONDQA/OPS/CDER/ FDA}

Quality by Design (QbD) is a science and risk based approach for product development. Enhanced process understanding gained from following a $\mathrm{QbD}$ based approach, is increasingly being used to develop and maintain analytical procedures. The aim of this presentation is to discuss pathways for implementation of the QbD approach for analytical procedure development and to offer a regulatory perspective regarding considerations for successful implementation of this approach.

\section{Sharmista Chatterjee}

Dr. Sharmista Chatterjee is the CMC Lead for QbD (Quality by Design) in the Office of New Drug Quality Assessment (ONDQA) at the FDA. Sharmista has been with the agency since 2006, and has been actively involved in FDA's QbD efforts including serving as the technical lead for the FDAEMA QbD pilot, reviewing and consulting on QbD based applications, and supporting development of $\mathrm{QbD}$ related review practices and policies. Her background is in chemical and biomedical engineering, with degrees from Indian Institute of Technology and Iowa State University. Prior to joining the agency in 2006, she spent around 10 years in industry. Her industry experience was primarily in process development and modeling in diverse industries that included energy, consumer goods, food and pharmaceuticals. 


\section{Friday, June 13}

\section{SESSION 10:}

\section{Current Topics in Drug Safety}

\section{Principles of ADME-Tox in Drug Discovery and Development}

Leanne L. Bedard, PhD, Principal and Consultant, Bedard ADME-Tox Solutions

The acronym "ADME-Tox or ADMET" refers to Absorption, Distribution, Metabolism, Excretion, and Toxicity. ADMET properties, in addition to efficacy, are critical determinants of whether a preclinical drug candidate will progress into clinical development and potentially become a marketed pharmaceutical product. ADMET is a discipline in pharmaceutical research that emerged in the 1990s and that brings together ADME scientists with medicinal chemists, pharmacologists, toxicologists and drug product formulators. Early assessment of the ADME properties of drug candidates in discovery phase has reduced failures in clinical trials due to poor PK properties from $40 \%$ in 1990 to $10 \%$ in the year 2000. Consequently, drug discovery efforts have invested heavily in front-loading early screening and optimization of ADME-Tox properties to minimize risks and reduce attrition during the drug development process. It is important to define, as early possible, the targeted product profile which outlines the desirable efficacy, dosing regimen, safety profile, and pharmaceutical properties to be achieved. Properties that make a lead compound 'drug-like' include: efficacious at drug target with desirable pharmacological effect, no drug-drug interactions, no undesirable off-target effects, suitable half-life for desired dosing regimen, no toxic metabolites, and large therapeutic index. This presentation will provide a general overview of the principles of ADME-Tox and their application at different phases in drug discovery and development. The subsequent presentations in this session will focus on specific aspects of drug safety, notably drug-drug interactions, reactive drug intermediates and genotoxicity evaluation of drugs and impurities.

\section{Leanne L. Bedard}

Leanne completed her MSc in Physical Organic Chemistry at Carleton University in Ottawa and a $\mathrm{PhD}$ in Toxicology in 2004 at Queen's University in Kingston, Ontario, where she was also a postdoctoral fellow until 2006. Her research focused on the molecular mechanisms of chemical-induced lung cancer, including the bioactivation of pulmonary carcinogens. Leanne began her career in pharmaceutical R\&D at Merck Frosst in Montréal in 2006 as a Senior Research Scientist in Preclinical Drug Metabolism and Pharmacokinetics (DMPK). She contributed to the progress of several drug discovery projects for the treatment of respiratory and infectious diseases, reducing late-stage attrition of drug candidates due to poor DMPK properties. Leanne was the recipient of a Special Achievement Award for her contribution to the discovery of a new drug candidate currently in Phase $2 \mathrm{~b}$ clinical trials for the treatment of HIV-1. Leanne then joined AstraZeneca R\&D Montréal as DMPK Drug Design Leader, specializing in the discovery of pain control therapies. In 2012, Leanne founded Bedard ADMETox Solutions, which provides consultation services in the areas of drug metabolism, pharmacokinetics and toxicology. As a consultant, Leanne works with small and virtual pharma and biotech companies, regulatory agencies and CROs. Leanne has also been active in the scientific community. She currently holds an adjunct appointment in the Department of Biomedical and Molecular Sciences at Queen's University (2007-) and was a Councilor on the Board of Directors of the Society of Toxicology of Canada (2009-2012). She is a member of the Roundtable of Toxicology Consultants and was recently elected as a Councilor on the executive committee of the Drug Discovery Specialty Section of the Society of Toxicology (USA). Leanne is the author of 14 publications in the peer-reviewed literature, including an invited mini-review. 


\section{Assessment \& Prediction of Drug-Drug Interaction Potential at Drug Discovery \& Development Stages - VX 222 Case Study}

Jean-François Lévesque, DMPK Department, Vertex Pharmaceutical Canada, Laval, QC

Jean-François Lévesque ${ }^{1}$, Sarah Robertson ${ }^{2}$ and Nathalie Chauret ${ }^{1}$

Early assessment and prediction of DDI potential is crucial to triage molecules at drug discovery stage, select drug candidates with higher probability of success, support IND/NDA submissions, and ultimately minimize risk for patient safety. A case study will be presented on the use of static as well as dynamic tools (SimCYP PBPK simulator) to predict retrospectively and prospectively DDI potential of VX-222, a selective inhibitor of HCV NS5B polymerase. In clinic, VX-222 has been shown to be a victim of CYP3A4 mediated drug interactions, a moderate CYP3A4 inducer and pharmacokinetic studies in cirrhotic patients highlighted exposure increase versus healthy volunteers. Based on in vitro and human in vivo data, a SimCYP compound profile was built for VX-222 and retrospective DDI and PK predictions in healthy volunteers and cirrhotic patients were performed to validate the model. The clinical PK profiles (Cmax, AUC) of VX-222 were accurately predicted both in healthy \& cirrhotic virtual populations using the SimCYP model. SimCYP DDI module enabled the prediction of VX-222 interactions with ketoconazole (perpetrator) as well as midazolam (victim). In the latter case, there was indication that CYP3A induction may be predominantly occurring in the gut. Using the validated SimCYP VX-222 compound profile, prospective simulations were conducted to provide guidance for future clinical studies.

\section{Jean-François Lévesque}

After completing a B.Sc. degree in Chemistry at the Université de Montréal, Jean-François Lévesque pursued graduate studies in Analytical Chemistry at the Canadian Doping Control Laboratory (INRSIAF) under the supervision of Pr Christiane Ayotte. His research focused on DHEA and androstenedione metabolism and lead to the identification of novel urinary biomarkers currently used by the WADA to detect signs of anabolic steroid abuse by athletes. Jean-François then joined Merck Frosst as a DMPK Scientist where he was responsible for solving ADME and drug interaction (DDI) issues, devising strategies, leading \& managing scientific teams and collaborating on multidisciplinary project core teams at lead optimization (LO) and development stages. Over his 11 years at Merck, Jean-François contributed to $>10$ LO programs across 5 different therapeutic areas. He actively participated to the discovery and profiling of over 20 preclinical drug candidates, including Tredaptive (EMEA) and Odanacatib (Phase III). Jean-François then joined the Faculty of Pharmacy at the Universite de Montréal as the Manager of the Biopharmacy Platform (ADME-Tox). He was responsible for the startup, development and management of the platform, which offers ADME-Tox services and support to partners in academia and industry. JeanFrançois is currently employed at Vertex Pharmaceutical Canada as a DMPK Research Fellow, where he develops and implements innovative DMPK strategies for programs targeting inflammatory bowel disease (IBD). He has a wide interest in human DDI prediction as well as drug absorption and distribution within GI track to support PKPD for IBD drug targets. Jean-François is the co-author of 30 publications in peer-review journals as well as one book chapter.

\section{Metabolic Activation and Reactive Metabolites}

R. Scott Obach, Senior Research Fellow, Pharmacokinetics, Dynamics, and Drug Metabolism Department, Pfizer Inc., Groton, CT, USA

Drugs can be converted to chemically reactive metabolites by drug metabolizing enzymes. Developing the link between generation of chemically reactive metabolites and toxicity is challenging. Retrospective analysis of instances of drug toxicity wherein the toxicity can be associated with reactive metabolites has been done for many examples, however to use reactive metabolite data in a prospective manner to predict which drugs may cause toxicity is extremely challenging. In drug research, in vitro drug metabolism assays such as the generation of nucleophile adducts of reactive metabolites in drug metabolism incubations or measurement of covalent binding to macromolecules in vitro have been used as measures of bioactivation and warning signs for potential toxicity. We and others have attempted to categorize drugs based on a combination of covalent binding rate in vitro and in vivo daily dose. This concept has been extended to the characterization of drugs that already have 
reactive electrophilic substituents (without metabolic activation). An "avoidance" strategy used in early drug design, based on structural alerts and in vitro drug metabolism data will be described. Until better knowledge becomes available that shows a quantitative and mechanistic cause-and-effect between covalent binding and toxicity, this will continue to be a daunting challenge to those engaged in the discovery and development of new medicines.

\section{R. Scott Obach}

Scott Obach is a Senior Research Fellow in the Pharmacokinetics, Dynamics, and Drug Metabolism Department at Pfizer in Groton, CT. He earned his $\mathrm{Ph} . \mathrm{D}$. in biochemistry from Brandeis University in 1990, followed by a post-doctoral fellowship in 1990-1992 at the New York State Department of Health Research Laboratories. In 1992, Scott joined the Drug Metabolism Department at Pfizer Inc. as a Research Scientist. He currently serves on the editorial boards of Drug Metabolism and Disposition, Chemical Research in Toxicology, Drug Metabolism and Pharmacokinetics, and Xenobiotica. $\mathrm{He}$ is an author or coauthor on over 150 research publications and has given invited oral presentations at over sixty scientific conferences. His research interests include application of in vitro approaches to study drug metabolism, prediction of human pharmacokinetics and drug interactions, mechanisms of cytochrome P450 catalysis and other biotransformation reactions, including generation of chemically reactive metabolites.

\section{Genotoxicity Evaluation of the API and Impurities: Current ICH Guidelines}

Robin M. Walker, PhD, DABT, CanBioPharma Consulting Inc.

Evaluation of potential genotoxicity is an important aspect in the nonclinical safety assessment of drugs and their impurities that is addressed in the revised International Conference on Harmonisation (ICH) "Guidance on Genotoxicity Testing and Data Interpretation for Pharmaceuticals Intended for Human Use (S2(R1))" and the new draft guideline "Assessment and Control of DNA Reactive (Mutagenic) Impurities in Pharmaceuticals to Limit Potential Genotoxic Risk (M7). The S2(R1) guideline provides 2 options. The first is conduct of a traditional 3 test battery consisting of bacterial mutagenicity, an in vitro mammalian cell cytogenetic assay, and an in vivo chromosome damage study. The new option consists of the bacterial assay and evaluation of 2 different tissues in an in vivo study. ICH M7 is an extension of previous EMA and FDA guidelines and largely supplants the ICH Q3A/B Guidelines with respect to addressing potential genotoxicity of impurities in drug substances and products if there are no existing genotoxicity/carcinogenicity data. Evaluation of genotoxic potential according to $\mathrm{Q} 3 \mathrm{~A} / \mathrm{B}$ (bacterial and in vitro mammalian assays) still needs to be considered if an impurity intake exceeds $1 \mathrm{mg} /$ day. ICH M7 identifies a generic "Threshold of Toxicologic Concern" (TTC) of $1.5 \mu \mathrm{g} / \mathrm{day}$ as an acceptable intake for most genotoxic impurities, since that dose is associated with a low lifetime risk of carcinogenicity $(1: 100,000)$ based on conservative extrapolation from existing rodent carcinogenicity data for many chemicals. Genotoxicity does not need to be evaluated for impurities at intakes below the TTC. Above the TTC, evaluation is required and ICH M7 establishes the primacy of the bacterial mutagenicity (Salmonella and $E$ coli) as the definitive assay. Potential genotoxicity can now be evaluated using in silico programs based on QSAR, where the activity data are from bacterial assays. Negative results from 2 complementary in silico programs (rule-based and statistical) are required to classify an impurity as non-genotoxic. However, negative findings in an appropriately conducted bacterial mutagenicity assay supersede positive in silico alerts. M7 extends the concept of the TTC, by stipulating that a value lower than the generic 1.5 $\mu \mathrm{g} /$ day would be indicated for particularly potent classes of carcinogens (aflatoxins, N-nitroso, \& azoxy structures), while higher TTCs can be justified if the drug is indicated for "Less-Than-Lifetime" duration or the indication is for severe or lifethreatening indications where treatment options are limited.

\section{Robin M. Walker}

Robin Walker is an American Board of Toxicology certified toxicologist with over 30 years experience in nonclinical aspects of drug development. After obtaining a $\mathrm{PhD}$ from the Department of Pathology in collaboration with the Department of Pharmacology and Toxicology at Queen's University, Robin worked for large pharmaceutical companies (Parke-Davis/Warner Lambert and Pfizer) for over 20 years and subsequently as a consultant to private companies and regulatory agencies through CanBioPharma Consulting Inc. 
Robin has extensive experience on international drug development teams with responsibilities for the design, overall direction, summarization, and issue resolution of nonclinical toxicology. He has been a member of highly successful development teams responsible for the development of marketed drugs (Neurontin and Cerebyx) and was responsible for the direction of exploratory and GLP-compliant laboratory operations that supported many drug development programs. Robin has been active in the Society of Toxicology of Canada (STC) serving as a councilor on the Board of Directors, member and chair of the Program Committee, and member of the Organizing Committee and co-chair of the Program Committee for the International Congress of Toxicology when it was held in Montreal. He also has extensive experience as a study director and monitor of contracted GLP-compliant toxicology studies. Robin has an extensive record of scientific publication that includes 32 peer-reviewed papers, 6 invited book chapters, and 65 meeting presentations. Consulting experience has been with respect to due diligence evaluations, development of preclinical development plans for small and medium sized pharmaceutical / biotechnology companies, preparation CTA/IND and other regulatory documents for new chemical entities and medical devices, monitoring of toxicology studies at contract laboratories, and provision of toxicology advice to industry and Health Canada. The latter has included evaluation of the safety of impurities in pharmaceuticals, particularly with respect to potential genotoxicity. He has also taught in the Regulatory Affairs Program at Humber College in Toronto and is an adjunct faculty member in the Department of Biomedical and Molecular Sciences at Queen's University in Kingston. 


\section{Friday, June 13}

SESSION 11:

\section{Recent Trends in Material and Biomaterial Research}

\section{Specific and Non-specific Interaction Forces at Electrified PEG-Functionalized Lipid-Bilayers}

Markus Valtiner, Max Planck Institute, Germany

Deciphering the contributions of specific and nonspecific interaction forces is important to understanding large-scale phenomena such as celladhesion to implant materials, bio-fouling and biocorrosion. Yet, binding properties of specifically functionalized macromolecules to metals are not fully understood at the molecular level. Here, we will discuss the interaction forces between a gold electrode surface and amine-functionalized polymers (PEG) using a novel electrochemical surface forces apparatus. We examined the potential dependence of specific amine-gold interactions and measured that the binding strength of amine-gold bonds can range from $0.5-40 \mathrm{kbT}$ per binding site, depending on the electrochemical potential. Notably, this interaction exhibits a pronounced minimum around the potential of zero charge, where the polymer-gold adhesion is dominated by non-specific interactions between the polymer backbone and electrode surface, consistent with the adhesion of PEG polymers with gold surfaces in the absence of amine functionalization. Further, repulsive hydration interactions were observed to be stronger for amine-terminated PEG compared to non-functionalized PEG, due to increased hydration and presence of counterions in the case of amine-terminated PEG. Our results provide molecular-scale insight into design and optimisation of specific/ non-specific binding for numerous applications requiring strong, specific binding interactions between polymers and metals (or their native oxides), for example passivating layers in biomedical implants.

\section{Markus Valtiner}

Markus Valtiner received his MSc (2005) and Phd (2008) from Vienna University of Technology. He was awarded with the Otto Hahn medal of the MaxPlanck Society in 2010. From 2009-2012 he was post-doctoral and Marie Curie international outgoing fellow working with Prof. Jacob Israelachvili at UC Santa Barbara. Currently, he is group leader at the Max Planck Institut für Eisenforschung, where he leads the group for "Interaction forces and Functional Materials". His group is working on chemistry and forces at electrochemical interfaces, single molecule force spectroscopy and protein materials.

\section{Polysaccharide-based Nanostructures for MRI Contrast Improvement}

V. Gaëlle Roullin, Pharmaceutical Nanotechnology Laboratory, Faculté de pharmacie, Université de Montréal (Qc, Canada)

Among the various available imaging modalities, Magnetic Resonance Imaging (MRI) plays a critical role in detection and diagnosis since it is noninvasive, it does not require the use of ionizing radiation and provides excellent contrast resolution. ${ }^{1}$ However, MRI suffers from a lack of sensitivity; information obtained from a simple unenhanced MR image is often not sufficient to highlight areas of interest. Thus, the contrast is often improved by administering contrast-enhancing agents (CAs). Currently, the most used agents are Gadoliniumbased complexes (Gd-CAs), which are able to generate an increase of intensity signals (positive contrast) of most abnormal tissues and hence facilitate the visualization of abnormal structures or lesions. $^{2} \quad$ However, their administration at high doses has been associated with the emergence of nephrogenic systemic fibrosis (NSF) in patients with renal failure. ${ }^{3}$ To decrease administered doses, we postulated that the entrapment of Gd-CAs in polysaccharide-based nanohydrogels would improve their paramagnetic efficacy. We therefore studied the loading of various commercial and commercially-derived Gd complexes into either a single polymer network (chitosan) or a hybrid polymer network (polyelectrolyte complexes of 
chitosan and hyaluronate). ${ }^{4}$ Results showed that it was possible to finely tune the relaxivity enhancement and obtain a noticeable improvement of MR images at 3T according to the gadolinium contrast agent and the hydrogel matrix composition. This could constitute a major step forward in the detection and grading of various pathologies, including brain lesions.

1 P. Caravan, Chem. Soc. Rev., 2006, 35, 512.

2 E. Terreno et al. Chem. Rev. 2010, 110, 3019; P. Hermann et al. Dalton Trans., 2008, 23, 3027; A. E. Merbach, L. Helm and E. Toth (Eds) The Chemistry of Contrast Agents in Medical Magnetic Resonance Imaging, Wiley and Sons, Chichester, 2nd edn., 2013.

3 J. M. Idée et al. Toxicology, 2008, 248, 77; A. Bertin et al. Toxicology in Vitro, 2010, 24, 1386.

4 T. Courant et al. Angewandte Chemie, 2012, 51(36), 9119. M. Callewaert et al. 2014, submitted.

\section{Gaëlle Roullin}

V Gaëlle Roullin obtained her Master degree in Chemistry-Biology interface from Université de Montpellier (France) in 1997 and a PhD in Pharmaceutical Sciences from Université d'Angers (France) in 2001. Her thesis work, realized under the supervision of Prof. Jean-Pierre Benoit, dealt with the treatment of malignant gliomas by stereotactic implantation of 5-fluorouracil-loaded microspheres. After a two-year postdoctoral fellowship in gene therapy at University Laval (Quebec) and a one-year postdoc position in protein delivery at Université Catholique de Louvain (Belgique), she was appointed assistant professor at Université de Reims Champagne Ardenne (France) in 2004. She started working on polymeric nanoparticles dedicated to MRI diagnosis and also on anticancer-loaded nanovectors. Recently (August 2013), she joined the faculty of pharmacy at Université de Montréal (Quebec). Her main research interests are based on polymeric theranostic nanosystems, combining the diagnostic abilities of MRI contrast agents to the therapeutic potencies of chemotherapeutic agents. More specifically, she initiated a program to better understand how the design of such nanovectors could address the problem of bypassing the bloodbrain barrier. She is also interested in designing and evaluating nanohydrogels aimed at delivering therapeutic peptides for articular joint therapy.

\section{Towards Biomimetic Three-dimensional Micro- environments: Biomaterials and Microfabrication Approach}

Shilpa Sant, Assistant Professor, Department of Pharmaceutical Sciences and Bioengineering, University of Pittsburgh

In vitro cell-based assays are widely used for screening drug safety and efficacy in the pharmaceutical research. However, traditional twodimensional (2D) cell culture techniques often fail to recapitulate microenvironmental context and relevant complexity of tissues in vivo. A tissueengineered model that recreates the three dimensional (3D) structure, cell-cell/cell-matrix interaction, and signalling cues present in vivo will dramatically improve the current preclinical drug testing paradigm. To create physiologically relevant 3D tissue models, it is important to engineer biomaterials with tailored physicochemical, mechanical and topographical cues and study cellcell and cell-matrix interactions. In this presentation, I will highlight how we can use expertise from microfabrication, tissue engineering and materials science to engineer biomimetic microenvironments in vitro that can further be useful in understanding pathophysiology of the disease, as well as developing regenerative therapies.

\section{Shilpa Sant}

Shilpa Sant, $\mathrm{PhD}$ is an Assistant Professor in the Department of Pharmaceutical Sciences and Bioengineering at the University of Pittsburgh. She is also a faculty member at McGowan Institute for Regenerative Medicine. Her research is focused on engineering biomaterial-based three-dimensional tissue models for drug discovery and regenerative therapies. Dr. Sant was Ruth Kirschstein NRSA Interdisciplinary fellow at the Wyss Institute for Biologically Inspired Engineering, Harvard University, and the Center for Bioengineering at Harvard Medical School in Boston, MA. She received her Ph.D. in Pharmaceutical Technology from University of Montreal, Canada, a Masters degree in Pharmacology and a Bachelors degree in Pharmaceutical Sciences from the University of Mumbai. She has extensive research experience in diverse fields including materials science, polymer chemistry, drug delivery, tissue engineering and microfabrication. Her postdoctoral research involved fabrication of functionalized bioinspired materials and scaffolds applicable for the heart valve and tooth 
germ tissue engineering. She has contributed over 23 articles in peer-reviewed journals, 5 book chapters, 4 abstracts and co-edied a book entitled "Nanomaterials in tissue engineering: Fabrication and applications". Dr. Sant is a recipient of many prestigious fellowships including Graduate Scholarship by the University of Montreal, Postgraduate Scholarship by Natural Sciences and Engineering Research Council (NSERC), Canada, Postdoctoral Research Fellowship from Le Fonds Quebecoise de Recherche sur Nature et Technologies (FQRNT), and Ruth L. Kirschstein National Research Service Award by NIH. She has also received various awards including "2013 CMBE-BMES Rising Star/Fellow Award" and "2010 Society for Biomaterials - STAR Award".

\section{Towards the Development of Bioadhesive Polymers Mimicking Myelin Basic Protein}

Xavier Banquy, Université de Montréal

Myelin dysfunctions are characterized by a change in the lipid composition of the myelin membrane which leads to the appearance of lesions reflecting loss of inter-membrane adhesion.

We hypothesize that these processes can be controlled or even reversed by modifying the molecular interactions between the lipids and proteins in the myelin membrane using bioadhesive polymers. By controlling the chemical composition and architecture of the polymers, we will show that it is possible to enhance the adhesion between membranes using different types of molecular interaction forces such as electrostatic forces, depletion forces, bridging forces or combinations of them.

The magnitude of these interaction forces depends on several physical-chemical parameters such as the concentration, the size and architecture of the polymer as well as the affinity of the polymer to the bilayer. The effect of these parameters on model myelin membranes adhesion will be presented and conclusions will be made about the possible use of these polymers as a possible "treatment" that could lead to the prevention or reversal of swelling and vacuolization of myelin.

\section{Xavier Banquy}

Xavier Banquy received his $\mathrm{PhD}$ in Pharmaceutical Sciences in 2009 under the supervision of $\mathrm{Pr}$ Suzanne Giasson at the University of Montréal. He joined Prof. Israelachvili's group at the University of California, Santa Barbara, as a post doctoral fellow. Since 2013, he is Assistant Professor at the faculty of pharmacy of the University of Montreal. His current research interests are focused on the development of structured biomaterials and surfaces for the treatment of degenerative diseases such as multiple sclerosis and osteoarthritis. 


\section{Poster Session 1}

\section{CSPS and CC-CRS Posters}

\section{Wednesday, June 11}




\section{Poster Session 1}

\section{Wednesday, June 11}

\section{Biomedical Sciences}

\section{The Effect of Advanced Age and Inflammation in Inducing Stroke in Hypertensive Situations}

Kaetan Waghmare, Amy Randell, Krista Squires, Noriko Daneshtalab

School of Pharmacy, Memorial University of Newfoundland, Health Sciences Centre, St. John's, NL, Canada

Introduction: We have previously established a hypertensive rat model with inflammation on young spontaneously hypertensive rats (SHR). However, we did not observe signs and symptoms of hemorrhagic stroke. Our goal was to determine whether degree of inflammation, and age plays a part on the degree of hypertension and severity of inflammation in the animal, and whether it increases the risk for hemorrhagic stroke development. The hypothesis is that with increasing age and hypertensive state, addition of increased inflammatory state will increase the risk of cerebrovascular dysfunction and consequently increase the potential for hemorrhagic stroke.

Materials/Methods: SHRs were fed a Japanese style high salt diet $(4 \% \mathrm{NaCl})$ from the time of weaning (5-6 weeks). Arthritis was induced by injection of manually compounded Complete Freunds Adjuvant (CFA) (0.1ml intradermally) into hind paw of SHRs which were 7-10 months of age. Control animals received saline injection. Blood pressures were measured weekly using tail vein plethysmography equipment from IITC. Ipsilateral paw and ankle were measured using digital calipers daily, alongside water displacement measurements. Experimental animals were monitored for signs and symptoms of stroke. At the end of the experiment, animals were sacrificed, organ and blood samples were collected and the brains analyzed.

Results and Discussion: The degree of inflammation was significantly higher with manually compounded CFA and injection dose required adjustment. The paws of SHRs injected with CFA were significantly inflamed with joint abnormalities as compared to saline injected SHRs. However, no physical signs or symptoms of stroke was observed in any of the experimental animals, similar to younger SHRs. Organ and brain examinations determined that there was brain edema and kidney dysfunction, analogous to experimental animals in post-stroke state.

Conclusion: Although there were clear signs of inflammation in the older SHRs with CFA treatment, none developed any physical signs or symptoms of stroke. However, further investigation on the cerebrovascular function is warranted, as brains of CFA-treated animals showed possible blood brain barrier permeability and edema; suggesting there may be some degree of vascular dysfunction, predisposing the SHR-CFA treated rats to hemorrhagic stroke development.

Acknowledgement: Kaetan Waghmare received the National Summer Student Research Program Awards to Present Research Findings sponsored by GlaxoSmithKline Inc.

\section{Novel Probiotic Dental Caries and Periodontal Disease Bio-Therapeutic}

$\underline{\text { Shyamali Saha }}^{1,2}$, Catherine Tomaro Duchesneau ${ }^{1}$, Meenakshi Malhotra ${ }^{1}$, Maryam Tabrizian ${ }^{1,2}$, Satya Prakash ${ }^{1}$.

${ }^{1}$ Biomedical Technology and Cell Therapy Research Laboratory, Department of Biomedical Engineering, Faculty of Medicine, McGill University, Montreal, Quebec; ${ }^{2}$ Faculty of Dentistry, McGill University, Montreal, Quebec, Canada

Purpose: Dental caries and periodontal diseases have high worldwide prevalence rates with almost $100 \%$ and $5-20 \%$ prevalence for adults, respectively. Current treatments focus on prevention and restoration of lost oral structures with sub-optimal success. With their complex/multifactorial etiology, 
the key therapeutic targets are pathogen inhibition, limiting inflammation and restoring lost tissue. The chronic nature of these diseases demand a safe longterm bio-therapeutic. Probiotic formulations have potential as oral health biotherapeutics. The goal of this study was to develop a novel probiotic dissolvable oral thin film as an oral disease biotherapeutic.

Methods: Screening of Lactobacillus strains were performed for the inhibition of Streptococcus mutans, a dental caries causing microorganism, using both qualitative agar and quantitative investigations under simulated oral conditions. Mechanistic studies were performed for nutrient (sucrose) competition, antioxidant activity and hydrogen peroxide production. Chronic inflammation and connective tissue loss are charactristics of severe periodontal diseases. Lactobacillus fermentum NCIMB 5221, demonstrating anti-oxidant capabilities, was investigated for promoting osteoblast growth and lowering pro-inflammatory cytokine secretion by inflamed osteoblasts. Lastly, a novel carboxymethyl cellulose (CMC)-oral thin film (OTF) was developed for probiotic delivery in the oral cavity.

Results: Tested Lactobacillus strains demonstrated S.mutans inhibition. Lactobacillus. reuteri NCIMB 701359 , L. reuteri NCIMB 701089 , L. reuteri NCIMB 702655 and L. reuteri NCIMB 703656 were the best $S$. mutans inhibitors, with no detectable colonies $(<10 \mathrm{cfu})$ after $24 \mathrm{~h}$. L. reuteri mediated S.mutans inhibition was correlated to $\mathrm{H}_{2} \mathrm{O}_{2}$ production, confirmed by catalase inactivation of $\mathrm{H}_{2} \mathrm{O}_{2}$. Moreover, L. fermentum NCIMB 5221 treatment reduced both IL-1 $\beta(p=0.00008)$ and IL-6 $(p=0.004))$ secretion by inflamed osteoblast-like cells. It also significantly promoted the growth of osteoblast-like cells $(p<0.005)$. Finally, we successfully developed novel OTFs using a natural polymer $\mathrm{CMC}$, suitable for delivery of probiotic bacteria to the oral cavity.

Conclusion: This study demonstrates the potential of probiotic bacteria for developing a natural and safe biotherapeutic for long-term use in chronic oral diseases.

Acknowledgement: The authors would like to acknowledge a Canadian Institute of Health Research (CIHR) grant (MOP 264308) to Dr. S. Prakash, an Alexander Graham Bell Canada Graduate Doctoral Scholarship from the NSERC to C. Tomaro-Duchesneau, and a FRSQ Doctoral Scholarship to M. Malhotra.

\section{Oxygen Delivery System for Tissue Growth}

Huaifa Zhang ${ }^{1}$, Faleh Tamimi Marino ${ }^{1}$, Svetlana Komarova $^{1}$, Jake Barralet ${ }^{1,2}$

${ }^{1}$ Faculty of Dentistry, McGill University, Montreal, QC; ${ }^{2}$ Division of Orthopedics, Department of Surgery, Faculty of Medicine, McGill University, Montreal, QC, Canada

Purpose: Hypoxia and even anoxia may occur during wound healing and tissue engineering. Oxygen supply is a major limitation for tissue growth since its absence induces cell apoptosis and necrosis. In this study we controlled the rate of oxygen delivery in a biomaterials based system and examined its effect on cell growth and function in anoxic culture.

Methods: $\mathrm{CaO}_{2}$ decomposes in water to generate $\mathrm{O}_{2}$ (Equation (1)). The byporoduct of this reaction is calcium hydroxide and also hydrogen peroxide that cause cytotoxicity. We used hydrophobic polymers combined with hydrogels to adjust the oxygen release rate and to mitigate the cytotoxic effect of the reaction.

$\mathrm{CaO}_{2}+2 \mathrm{H}_{2} \mathrm{O}=\mathrm{Ca}(\mathrm{OH})_{2}+\mathrm{H}_{2} \mathrm{O}_{2}$ $2 \mathrm{H}_{2} \mathrm{O}_{2}=2 \mathrm{H}_{2} \mathrm{O}+\mathrm{O}_{2}$

The $\mathrm{O}_{2}$ release system was prepared by encapsulating $\mathrm{CaO}_{2}$ powders or composites containing $\mathrm{CaO}_{2}$ in $3 \mathrm{wt} \%$ alginate hydrogel. $\mathrm{O}_{2}$ release behavior of the materials as a function of time was recorded using a Clark-type electrode. Primary human fibroblasts, cultured with the $\mathrm{CaO}_{2}-$ polymer-hydrogel under anoxia, were used to evaluate $\mathrm{O}_{2}$ release capacity and biocompatibility of the material. Anoxic culture without the material was carried out as the negative control. Normoxia culture was taken as the positive control. Cell viability, apoptosis and proliferation behaviors of the cells as well as their gene expression levels for Glut 1, BNIP 3 and VEGF related genes, markers of glycolysis, apoptosis and angiogenesis, respectively, were studied.

Results: Cell viability drastically decreased during anoxic culture (Figure 1). Nevertheless, the cells cultured under anoxic conditions in presence of our $\mathrm{O}_{2}$ release material maintained a remarkable growth rate similar with that of cells cultured under normoxia. The $\mathrm{O}_{2}$ release material protected cells from apoptosis and maintained their normal mitosis activities. The expression levels of the genes related to glycolysis, apoptosis and angiogenesis were reduced by the material under anoxia compared with negative control (Table 1). 


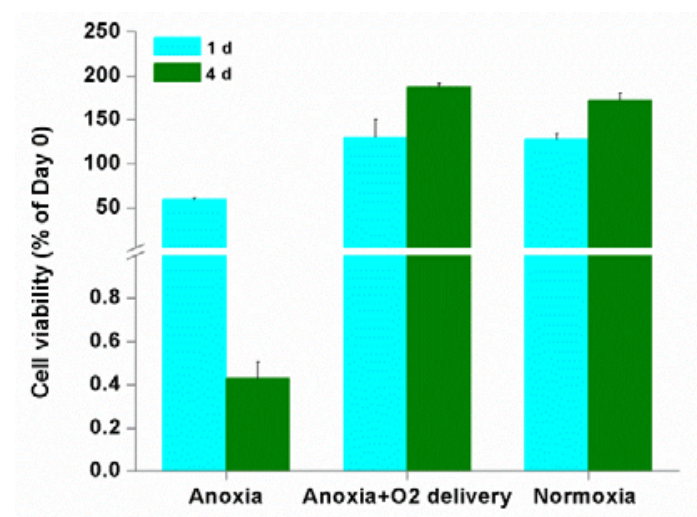

Figure 1 Cell viability results under different conditions (Alamar Blue staining).

Table 1. Expression levels of genes (normalized to normoxic culture)

\begin{tabular}{lll|ll}
\hline & \multicolumn{2}{c|}{$1 \mathrm{~d}$} & \multicolumn{2}{c}{$4 \mathrm{~d}$} \\
\cline { 2 - 5 } & $\begin{array}{l}\text { Anoxia+ } \\
\mathrm{O}_{2}\end{array}$ & Anoxia & $\begin{array}{l}\text { Anoxia }+ \\
\mathrm{O}_{2}\end{array}$ & Anoxia \\
\cline { 2 - 5 } Glut & & $12.2 \pm 1$. & $1.6 \pm 0.3$ & $6.8 \pm 0.1$ \\
1 & $1.7 \pm 0.84$ & 41 & 3 & 8 \\
BNI & $2.8 \pm 0.94$ & $11.9 \pm 3$. & $4.5 \pm 0.5$ & $20.8 \pm 2$. \\
P 3 & $1.4 \pm 0.88$ & 60 & 8 & 50 \\
VEG & & $11.5 \pm 4$. & $2.3 \pm 0.2$ & $6.6 \pm 2.1$ \\
F & & 04 & 8 & 6 \\
\hline
\end{tabular}

Conclusion: A biodegradable in-situ $\mathrm{O}_{2}$ delivery material for tissue engineering was developed with tunable gas release rate. This new material demonstrated outstanding capacities in rescuing primary human fibroblasts as well as in keeping normal cellular metabolic activities under anoxic environment.

\section{References}

1. Carreau, A., et al., J. Cell. Mol. Med., 2011. 15(6): p. 1239-1253.

2. Lovett, M., et al., Tissue Eng. Part B: Reviews, 2009. 15(3): p. 353-370.

3. Pedraza, E., et al., Proc. Natl. Acad. Sci. U.S.A, 2012. 109(11): p. 4245-4250.

\section{The Effect of Local Delivery of Hypoxia Mimics on Biocemeraic Bone Graft Remodeling}

Justin Drager ${ }^{1}$, Zeeshan Sheikh ${ }^{2}$, Edward Harvey ${ }^{1}$, Jake Barralet ${ }^{1,2}$

${ }^{1}$ Devision of Orthopedic Surgery, McGill University Health Center, Montreal, Quebec; ${ }^{2}$ Faculty of Dentistry, McGill University, Montreal Quebec

Purpose: Hypoxia Inducible Factor- 1a (HIF-1a) signaling is intricately involved in bone biology. Activation of this pathway in response to hypoxic conditions stimulates angiogenesis, progenitor cell recruitment and has direct effects on differentiation and activity osteoblasts and osteoclasts. Interestingly, several small molecules such as the widely used iron chelator Deferoxamine (DFO) have been identified as activators of the HIF-1a gene and have been shown to induce vascular growth and bone formation when locally injected into fracture sites. The aim of this study was to determine the effect of sustained local delivery of DFO on bioceramic bone graft remodeling in a rabbit cranial onley model.

Methods: Cylindrical Monetite grafts $(9 \mathrm{~mm}$ diameter/ $4 \mathrm{~mm}$ in height) were implanted subperiosteally onto each cranium of six NewZeeland White Rabbits. Starting on post-operative day 4, DFO (500ul of 200uM solution) or saline was injected subcutaneously into the graft area every $48 \mathrm{hr}$ for total of 6 does. Three rabbits were subject DFO injections while three had control saline injections. Live animal CT followed the healing progression over 8 weeks. After explanation graft resorpiton and bone ingrowth was assessed by micro-CT and hard tissue histology.

Results: Qualitative assessment of CT images demonstrated progressive integration at the bonegraft interface in the DFO group but not the saline group. Micro-CT analysis showed significantly less graft resoption in the DFO group compared to saline controls. Coronal histological cuts confirmed increased graft resorption and new bone formation in the saline group. However the pattern of growth differed between the groups, with the DFO group exhibiting more prominent trabeculations extending from the calvaria. In contrast the saline group exhibited de-nevo bone forming along the areas of graft remnant with few clear connection to the calvaria. Soft tissue had fully encapsulated the grafts in the DFO group and the majority of the graft pores were infiltrated by soft tissue cells. TRAP staining 
for osteoclasts demonstrated similar numbers in both groups.

Conclusion: Local delivery of DFO reduced the in vivo resorption of calcium phosphate grafts subsequently leading to lower bone infiltration. However thicker trabelculae of bone extending from the skull was evident in the DFO group. Reduced resorption was unlikely due to decreased osteoclast recruitment but possibly related to more soft tissue infiltration within the grafts. These results somewhat conflict with data on DFO effects in long bone models. The possibly that the two bone types respond differently to hypoxia mimics remains to be determined.

\section{Study on the Potential Effect of Lactobacillus Reuteri in Inhibiting Colon Cancer Cells and the Role of Short Chain Fatty Acids Production: In-Vitro Analysis}

Imen Kahouli ${ }^{1}$, Meenakshi Malhotra ${ }^{1}$, Catherine Tomaro-Duchesneau $^{1}$, Shyamali Saha, ${ }^{1,3}$, Daniel Marinescu $^{1}$, Laëtitia Sonia Rodes ${ }^{1}$, Moulay A. Aloui-Jamali ${ }^{1,2}$ and Satya Prakash ${ }^{1 *}$

${ }^{1}$ Faculty of Medicine, McGill University, Montreal, QC; ${ }^{4}$ Lady Davis Institute for Medical Research and Segal Cancer Centre, Sir Mortimer B. Davis-Jewish General Hospital, Montreal, QC; ${ }^{5}$ Faculty of Dentistry, McGill University, Montreal, QC, Canada

Introduction: While Probiotics has been suggested as chemopreventive agents against colorectal cancer (CRC) and are consumed widely as health supplement by patient and healthy individuals, more investigations are needed on the potential colonic health effects of specific probiotic bacteria, such as Lactobacillus reuteri, to be used in CRC.

Materials and Methods: Five L. reuteri strains were compared according to their production of SCFAs and on their effect on cancer cell growth (Caco-2). The composition of SCFAs and lactic acid produced by bacteria in $\mathrm{CM}$ was used to prepare "synthetic SCFAs formulations". Similarly, the antiproliferative activity of these synthetic formulations on cancer cells was compared with probiotic CM. Then, the stability of some $L$. reuteri strains in an intestinal environment and their biological activity, described by the production of SCFAs, in a simulated intestinal fluids (SIFs), was used. For more characterization Free fatty acid profile of a selected bacteria and their bioactivity was compared to other species. The anti-proliferative effect of the probiotic supernatants towards colon cancer cells and normal cells was investigated. The antiproliferative effect of reconstituted SCFA formulations was determined.

Results and Discussion: The results showed that the production of SCFAs in the $\mathrm{CM}$ was strain dependent and that $L$. reuteri NCIMB 11951, 701359 and 702656 produced the highest amount of total SCFAs $(402.2 \pm 23.5 \mathrm{mg} / \mathrm{L}, p<0.01)$. Similarly, the same strains of $L$. reuteri inhibited the best Caco-2 cancer cell proliferation in vitro (by $56.7 \pm$ $1.6 \%$ compared to non-treated cells, 72 hours). Subsequently, SCFAs formulations corresponding to these strains were also the best at inhibiting CRC growth, however significantly with less effect (maximum $24.15 \pm 1,03 \%$ of cell inhibition, $\mathrm{p}<0.001)$. The selected strains $L$. reuteri NCIMB 702656 and 701359 showed higher resistance to a simulated intestinal environment (minimum 94,91 \pm $0.59 \%$ of viability, 4 hours) and produced more SCFAs in SIF (total SCFAs: $1391.58 \pm 4.84 \mathrm{mg} / \mathrm{L}$ ). L. reuteri NCIMB 701359 was more bioactive and produced more FFA. Probiotic treatments showed inhibitory effects cancer cells but not on normal cells.

Conclusion: $L$. reuteri strains, mainly $L$. reuteri NCIMB 701359 as a PA-producer bacterium, have a better potential to be an effective agent in biotherapeutic strategy for cancer prevention.

\section{Pharmaceutical \& Analytical Chemistry}

\section{Optimization of a PAMPA-BBB Model and Subsequent Validation by a Consolidated LC- MS/MS Method}

$\underline{\text { Florian Bernard }}^{1,2}$, Pierre-Luc Latreille ${ }^{3}$, Martin Jutras $^{2}$, Mihaela Friciu' ${ }^{2}$, V Gaëlle Roullin ${ }^{1}$, and Grégoire Leclair $^{2}$

${ }^{1}$ Pharmaceutical Nanotechnology Laboratory; ${ }^{2}$ Platform of Biopharmacy; and ${ }^{3}$ Biomaterials and Structured Interfaces Laboratory, Faculté de Pharmacie / School of Pharmacy, Université de Montréal, Montreal QC, Canada

Purpose: The PAMPA-BBB assay is used to simultaneously screen a high number of drugs 
potentially aimed at the central nervous system. Indeed it allows discriminating molecules seemingly able to passively diffuse the Blood-Brain Barrier (BBB) in vivo (1). However, its set up is critical to obtain quality results and relevant data (2).

Aims of this study were (i) to optimize several PAMPA-BBB settings; (ii) to elaborate a multi-drug cassette serving as controls for this assay; (iii) to develop and validate a LC-MS/MS method for the simultaneous quantification of this drug cassette.

Methods: Various drugs were examined to constitute the multi-drug cassette. Different criteria were applied to discriminate them, among which relevance to $\mathrm{BBB}$ passage and predicted $\log \mathrm{D}$.

The PAMPA-BBB protocol was adapted according to previous studies (3). Briefly, a filter plate $(0.45$ $\mu \mathrm{m}$, hydrophobic filter PVDF, EMD Millipore, USA) was coated with a $22 \mathrm{mg} \mathrm{mL}^{-1}$ porcine brain polar lipid solution (PBL, Aventi Polar Lipid Inc, USA). Permeability was determined by incubating the chosen multi-drug cassette solution in the donor compartment and then retrieving the receptor compartment. Several parameters were optimized in terms of time, delay, concentration and temperature (4). An HPLC 1100 series (Agilent technologies, Canada) paired with a Sciex API4000 QTRAP triple quadrupole (USA) was used for sample analysis. The ionization source was a turbo electrospray ionization (ESI), scan mode was a multiple reaction monitoring (MRM) and dwell time was set at 70 msec.

Results: The multi-drug cassette was narrowed to six molecules: midazolam, tenoxicam, caffeine, nicotine, atenolol and theophylline. The main selection criteria was the predicted Log D at $\mathrm{pH} 7.4$ $(-0.63-3.23)$; additional ones were molecular weight (162- 337 g. $\left.\mathrm{mol}^{-1}\right)$, permeability efficiency $\left.\left(0.07-9.80 \times 10^{-6} \mathrm{~cm}^{-1}\right)^{-1}\right)$ and able / unable to pass the BBB in vivo. Subsequently, the PAMPA-BBB assay was successfully optimized and the multi-drug cassette was used to validate the selected settings. To quantify the passive diffusion ability, each drug in its optimal concentration was dosed both in the donor and receptor compartments. The validation of the LC-MS/MS method was performed by choosing a relevant internal standard (metoclopramide) and then testing the selected drug cassette for LLOD, LLOQ, linearity, accuracy and precision (Table 1).

Conclusion: The optimized PAMPA-BBB assay, coupled with a validated LC-MS/MS method, allowed the rapid and accurate simultaneous quantification of six standard drugs. On-going statistical correlation should allow determining the most important criteria for predicting passive BBB diffusion.

\section{Validation Method for the Quantitation of Seven Compounds used in Ophthalmology in Multiple Simulated Lachrymal Fluids Using Liquid Chromatography Tandem Mass Spectrometry}

Pierre-Luc Latreille $^{1}$, Xavier Banquy ${ }^{1}$

${ }^{1}$ Chaire de recherche du Canada sur les matériaux inspirés du vivant, Faculté de Pharmacie, Université de Montréal, Montréal, QC, Canada

Purpose: In pharmaceutical science to achieve important studies, the use of at least one drug must be implied and an analytical method is usually developed to perform concentration quantitation of that drug in different complex matrixes such as buffer, blood and tissues. Since the LC-MS/MS has been established as the gold standard to perform these bioanalytical analysis we decided to develop a method to simultaneously quantitate seven compounds used in ophthalmology. In this way we wanted to quantify pilocarpine, lidocaine, proparacaine, triamcinolone, atropine, timolol and prednisolone in different intra-humoral / intravitreous like matrixes to perform controlled release study in vitro and in vivo.

Methodology: Using a Agilent series 1100 HPLC system coupled with an AB Sciex 4000 QTrap mass spectrometer with Analyst 1.4 software. The seven compound have been optimized with their internal standard for their optimum MS/MS parameter detection such as declustering potential (DP), collision energy (CE), and their optimum molecular

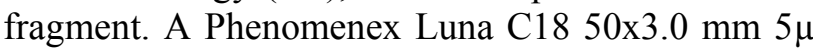
column and a gradient of water buffered with 10 $\mathrm{mM}$ ammonium acetate [A] and methanol: water (95:5) with $0.1 \%$ formic acid (FA) [B], from $95 \% \mathrm{~A}$ to $2 \% \mathrm{~A}$ in 3.5 minutes with a equilibration time of 1.4 minute, was used to analyse samples. Calibration curve from $0.25 \mathrm{nM}$ to $1000 \mathrm{nM}$ have been tested with different matrices such as solvent $(80 \%$ water, $20 \%$ ACN, $0.1 \%$ FA), simulated lachrymal fluids ( $80 \%$ saline solution, $20 \% \mathrm{ACN}$ ), and with a bovine serum albumin (BSA) based simulated lachrymal fluid $(80 \%$ water - salts $-2.5 \mathrm{mg} / \mathrm{mL}$ BSA, $20 \%$ $\mathrm{ACN})$.

Results: Calibration curves showed a $\mathrm{R}^{2}$ higher than 0.99 for each compound and showed low or nonsignificant matrix effect compared with solvent. A 
method using signal-to-noise ratio presented a $0.25 \mathrm{nM}-2.5 \mathrm{nM}$ limit of detection and $0.25 \mathrm{nM}-5.0$ $\mathrm{nM}$ limit of quantitation. Upper limit of quantitation varied between $250 \mathrm{nM}$ to $1000 \mathrm{nM}$. Results show that this bioanalytical method is robust enough according to FDA guidelines and ICH.

Conclusion: Following FDA and ICH guidelines, this proves that this method is robust enough to allow the quantitation of simultaneous compounds either to shorten the analysis time or viewing release of two or more compounds at the same time. As result, it will be possible to achieve these analyses in vivo with intra-humoral or intra-vitreous sampling from rodents.

\section{Development of Absolute Quantitative Analysis of Multiple Bile Acids Using LC/MS/MS}

Joanna Zheng, Petia Shipkova, Baomin Xin, and Timothy Olah

Bioanalytical Research, Bristol Myers Squibb Company, Pennington, New Jersey, United States

Purpose: Bile acids (BAs) are steroid acids found predominantly in bile of mammals. Bile acids play important roles in digestion, modulation of gut microbiota, and regulation of pathways necessary for cholesterol, lipid and glucose homeostasis. Bile acids have potent toxic properties (e.g., membrane disruption) and there are a plethora of mechanisms to limit their accumulation in blood and tissues. Elevated concentrations of bile acids in peripheral fluids can therefore be used as an indicator of various diseases. Since BAs are used as biomarkers in some disease model including liver toxicity, a robust quantification method is very critical in the assessment of BA levels.

Method: There are many bile acids and the distinctions between different bile acids are minute, depending only on the presence or absence of hydroxyl groups on positions 3,7 , and 12 . The quantitation of bile acids are challenging since they are endogenously present and variable in different species. In this presentation, the authors will report an unique assay using stable isotope labeled (SIL) bile acids as standards. Seven deuterated bile acids were spiked into animal plasma to make standard curves to avoid the interference from endogenous bile acids.

Results: An LC/MS/MS method is developed to quantify seven different bile acids together with their stereo-isomers (20 bile acids) in biological fluids. The LOQ for most bile acids is $2 \mathrm{ng} / \mathrm{mL}$. A four minute analytical run capable of separating all the isomers chromatographically provides the method the high throughput capacity.

Conclusion: A single sensitive and specific LC/MS/MS method capable of detecting and separating 20 bile acids plus seven SIL bile acids is developed. The method can be applied to support various drug discovery and development studies.

\section{Stability of Four Compounded Preparations of Tranexamic Acid and Alpha Thrombin}

Isabelle St-Jean ${ }^{1}$, Georges-Étienne Rivard ${ }^{2}$, JeanMarc Forest ${ }^{2}$ and Grégoire Leclair ${ }^{1}$

${ }^{1}$ Faculty of pharmacy, University of Montreal, Montreal, Quebec; ${ }^{2}$ Ste-Justine Pediatric Hospital, Montreal, Quebec

Purpose: Four compounded preparations containing tranexamic acid and alpha thrombin were developed at Ste-Justine Pediatric Hospital in Montreal, Canada for the topical treatment of epistaxis and other haemorrhages. The stability of these preparations was evaluated.

Methods: Topical hydrogel \#1 was prepared from tranexamic acid injection and KY jelly at a concentration of $1 \% \mathrm{w} / \mathrm{w}$; topical hydrogel \#2 was similarly prepared using tranexamic acid tablets and Intrasite gel; oral suspension \#3 was prepared from tranexamic acid tablets and simple syrup at a concentration of $100 \mathrm{mg} / \mathrm{mL}$; and topical hydrogel \#4 was similar to hydrogel \#1 with the addition of alpha thrombin $(120 \mathrm{IU} / \mathrm{g})$. Each preparation was triplicated and stored at $22^{\circ} \mathrm{C}$. Hydrogel \#4 was also stored at $-20^{\circ} \mathrm{C}$ and $5^{\circ} \mathrm{C}$. Tranexamic acid concentration was assessed by LC/MS/MS while alpha thrombin activity was evaluated by thrombin time bioassay.

Results: Tranexamic acid in each formulation was stable for at least 90 days, as the percentage of initial concentration and its $95 \%$ confidence interval remained above $90 \%$ for the entire duration of the study. Alpha thrombin also remained stable for 90 days when stored at $-20^{\circ} \mathrm{C}$ and $5^{\circ} \mathrm{C}$, as it maintained more than $90 \%$ of its initial activity. Besides, when stored at $22^{\circ} \mathrm{C}$, alpha thrombin was stable for at least 7 days; its stability could not be statistically confirmed after 14 days.

Conclusion: Tranexamic acid was very stable in the formulations. Hydrogel $\# 1$ and $\# 2$, as well as 
suspension \#3 maintained an acceptable assay for a period of at least 90 days at $22^{\circ} \mathrm{C}$. The presence of alpha thrombin in hydrogel \#4 reduced the stability of this preparation to 7 days at $22^{\circ} \mathrm{C}$. Refrigeration is recommended for this latter preparation as it remained stable for at least 90 days when stored at $5^{\circ} \mathrm{C}$ and $-20^{\circ} \mathrm{C}$.

\section{Pharmacokinetics \& Pharmacodynamics}

\section{Contribution of Pinocembrin Enantiomers to Potential Stereospecific Differential Pharmacokinetic and Pharmacodynamic Behaviour}

Casey L. Sayre, Stephanie E. Martinez, Bolanle Akinwumi, Hope D. Anderson, and Neal M. Davies Faculty of Pharmacy, University of Manitoba, Winnipeg, Manitoba, Canada

Purpose: Using a LC/MS method to quantify pinocembrin enantiomers; examine the stereoseletive pharmacokinetics of pinocembrin in rats; and evaluate the in vitro alpha amylase inhibitiory, antioxidant, and cardioprotective capacity of racemic pinocembrin and its two enantiomers.

Methods: LC/MS quantification method was performed using a Chiralpak $^{\circledR}$ AD-RH column. Pinocembrin enantiomers and the internal standard (7-ethoxycoumarin) were monitored in SIM positive mode at $\mathrm{m} / \mathrm{z} 255$ and $\mathrm{m} / \mathrm{z} 191$, respectively. The mobile phase was methanol and ammonium acetate $(100: 0.1 \mathrm{M} \mathrm{v}: \mathrm{v})$ with a flow rate of $0.5 \mathrm{ml} / \mathrm{min}$. Racemic pinocembrin was administered intravenously $(10 \mathrm{mg} / \mathrm{kg})$ and orally $(100 \mathrm{mg} / \mathrm{kg})$ to cannulated male Sprague Dawley rats $(\sim 250 \mathrm{~g}, \mathrm{n}=8)$. Serum and urine samples were collected over 72 hours post-dose. Alpha-amylase inhibition was assessed using a simple colorimetric assay with racemic pinocembrin and its enantiomers $(0-200$ $\mu \mathrm{g} / \mathrm{mL})$. Anti-oxidant activity of racemic and Spinocembrin $(0-200 \mu \mathrm{g} / \mathrm{mL})$ was measured using the ABTS method. Cardiomyoctyes were isolated from neonatal rats and treated with endothelin-1 to induce hypertrophy. Effects of pinocembrin and its enantiomers $(0-100 \mu \mathrm{g} / \mathrm{mL})$ were then evaluated. Cells were immunostained and measured for size using computer-assisted planimetry.

Results: Pinocembrin enantiomers were detected in plasma and urine primarily as glucoroconjugates. Pinocembrin enantiomers showed similar pharmacokinetic behaviour with respect to serum distribution and urinary excretion. After IV administration of racemic pinocembrin, the predominant pinocembrin enantiomer was in the Rconfiguration. Urinary excretion was $0.4 \%$ for Spinocembrin and $0.5 \%$ for R-pinocembrin. Absolute bioavailability was $\sim 28 \%$. Both enantiomers of pinocembrin appear to additively contribute to racemic pinocembrin alpha amylase inhibition. Preliminary data shows that racemic pinocembrin prevents cardiomyocyte hypertrophy in vitro with little contribution from S-pinocembrin. Spinocembrin showed greater concentrationdependent anti-oxidant activity than racemic pinocembrin.

Conclusion: The LC/MS assay is sensitive, reproducible, accurate, and specific. Pinocembrin disposition involves the rapid formation of a glucuronide metabolite and subsequent predominantly non-renal excretion. Distribution and excretion of pinocembrin enantiomers does not appear to be enantioselective. Pinocembrin enantiomers demonstrate dose dependent alpha amylase, antioxidant activity, and cardioprotective activity with variable contributions of the observed bioactivity of the pinocembrin enantiomers.

\section{Effect of Glycine on Cognitive Functions of 3xTg-AD Mice}

Jessica Virgili ${ }^{1}$, Milène Vandal ${ }^{1,2}$, Isabelle StAmour $^{1,2}$, Frédéric Calon ${ }^{1,2}$

${ }^{1}$ Faculty of Pharmacy, Laval University, ${ }^{2}$ CHUL Research Center (CHUQ)

Background: Alzheimer's disease (AD) is the most common neurodegenerative disease. It is characterized by impaired cognitive functions such as memory and learning. The N-methyl-D-aspartate (NMDA) receptors play a crucial role in learning and memory and their function is altered in $\mathrm{AD}$. Glycine is a brain-penetrant NMDA receptor coagonist frequently used as a vehicle for immunoglobulin preparations. Consequently, an exogenous intake of this amino acid could modulate the NMDA receptors response.

Purpose: This study aimed to verify the effects of a high dose of glycine on cognitive functions of in the 
3xTg-AD mouse, an animal model of $\mathrm{AD}$, which reproduces tau and $A \beta$ pathologies, the two main neuropathological markers of AD.

Methods: Two groups of 12-month-old 3xTg-AD male mice and non-transgenic controls received an intraperitoneal injection of glycine or saline twice a week (approximately $0.2 \mathrm{~g} / \mathrm{kg}$ ) for 4 months. Behavioural tests were performed within a week before their sacrifice at 16 months of age.

Results: The general locomotor activity, measured with the open field test, was similar between the groups. The dark-light box emergence test was used to assess anxiety-like behaviour and showed no difference between groups. With the exception of $3 \times T g-A D$ mice treated with glycine, all groups of mice recognized the new object (versus random $50 \%, \mathrm{p}<0.05$ ), suggesting that glycine impairs object recognition memory. Finally, preliminary qualitative analyses of $A \beta$ and tau pathologies performed by immunofluorescence in hippocampus sections showed no treatment-induced changes.

Conclusion: Glycine appears to induce a cognitive decline in 3xTg-AD mice. Further work on the effect of glycine on cognition and AD-like neuropathology is needed.

Acknowledgement: Jessica Virgili received the National Summer Student Research Program Awards to Present Research Findings sponsored by GlaxoSmithKline Inc.

\section{Pharmacokinetic Profile of a New High- Strength Fixed-Dose Combination of Ibuprofen/Methocarbamol 400/500 mg Tablet in Comparison to Single-component in the Presence and Absence of Food}

Alexandre Brkovic ${ }^{1}$, Gianfranca Piccirilli ${ }^{1}$, Marc Lefebvre $^{2}$ and Adrien Musuku ${ }^{1}$

${ }^{1}$ Pharmascience Inc., Montreal, Quebec, Canada,

${ }^{2}$ Algorithme Pharma, Laval, Quebec, Canada

Purpose: The objectives of this study were to compare the pharmacokinetic profile of a new combination product of ibuprofen/methocarbamol $400 / 500 \mathrm{mg}$ under fasting and fed conditions and to determine bioequivalence of this new combination product versus $400 \mathrm{mg}$ tablet of ibuprofen coadministered with $500 \mathrm{mg}$ methocarbamol tablet under fasting conditions. Safety was also assessed.

Method: In a single-center, three-period, crossover study, 36 healthy male subjects were randomized to receive a single dose of a combination product
400/500 mg tablet ibuprofen/methocarbamol under fasting conditions, after a high-fat, high-calorie breakfast and a single dose of $400 \mathrm{mg}$ tablet of ibuprofen co-administered with $500 \quad \mathrm{mg}$ methocarbamol under fasting conditions separated by 7 days of washout between each period. Plasma samples taken pre-dose and at regular intervals up to 10 hours post-dose were assayed for ibuprofen and methocarbamol by LC-MS/MS methods. Pharmacokinetic parameters were calculated using non-compartmental analysis. Analysis of variance was performed on log-transformed data. The formulations (combination product versus individual products) were to be considered bioequivalent and an absence of food-effect was to be considered (combination product fed/fasting) if the $90 \%$ confidence intervals $(\mathrm{CI})$ for geometric mean ratios (GMRs) for $\mathrm{AUC}_{\mathrm{T}}$ were between $80 \%$ and $125 \%$ and for $\mathrm{C}_{\max }$ the point estimate (PE) was to be within $80 \%$ to $125 \%$. For the food-effect assessment, the $\mathrm{T}_{\max }$ was compared based on rank transformation.

Results: Under fasting conditions, the $90 \%$ CI for $\mathrm{C}_{\max }$ and $\mathrm{AUC}_{\mathrm{T}}$ for both ibuprofen and methocarbamol were within $80 \%$ to $125 \%$ when comparing the combination product with $400 \mathrm{mg}$ ibuprofen tablet co-administered with $500 \mathrm{mg}$ methocarbamol tablet. Under fed conditions, methocarbamol $\mathrm{AUC}_{\mathrm{T}}$ and $\mathrm{C}_{\max }$ were reduced by $21 \%$ and $38 \%$, respectively, while foribuprofen, food reduced $\mathrm{C}_{\max }$ by $23 \%$, but did not significantly impact its extent of absorption. A significantly delayed $\mathrm{T}_{\max }$ was observed for methocarbamol and ibuprofen under fed conditions. The drugs products were well tolerated.

Conclusion: The combination product $(400 / 500 \mathrm{mg}$ ibuprofen/methocarbamol tablet) proved to be bioequivalent to $400 \mathrm{mg}$ ibuprofen tablet coadministered with $500 \mathrm{mg}$ methocarbamol tablet under fasting conditions. Food significantly affected the pharmacokinetic profile of methocarbamol.

\section{Coenzyme Q10 Lowers Blood Pressure and Heart Rate and Increases Red Blood Cell Concentrations of ATP in a Freely Moving Rat Model}

Patrick Tillman, Remigius U. Agu and Pollen K. Yeung

Pharmacokinetics and Metabolism Laboratory, College of Pharmacy and Department of Medicine, Dalhousie University, Halifax, NS, Canada 
Purpose: Coenzyme Q10 (CoQ10) is a naturally occurring anti-oxidant increasingly used in complementary medicine to enhance cardiovascular health. . The objective of the research was to study the effect of CoQ10 on cardiovascular hemodynamics and red blood cell (RBC) ATP concentrations.

Methods: Normotensive Sprague Dawley rats (SDR) with a carotid artery catheter weighing between 250 and $300 \mathrm{~g}$ were used. Each rat was housed in a freely -moving- caging environment with free access to drinking water. CoQ10 was dissolved in a vehicle made up of dimethyl sulfoxide (DMSO) and normal saline (1:1) for injection (5 $\mathrm{mg} / \mathrm{mL}$ ). Each rat received either $10 \mathrm{mg} / \mathrm{kg}$ of CoQ10 or the vehicle ( $\mathrm{n}=8$ in each group) twice daily for 4 doses by subcutaneous (sc) injection. Blood samples $(0.3 \mathrm{~mL}$ each) were collected at 0 (before the last dose), and at 0.08, 0.25 and 1 hour after the last dose for measurement of RBC concentrations of ATP by a validated HPLC. Hemodynamic recordings (SBP, DBP and HR) were collected continuously throughout the experiment. Data between the two groups (i.e. $\mathrm{T}$ and $\mathrm{C}$ ) were compared and differences considered significant at $\mathrm{p}$ $<0.05$ (Student's t-test).

Results: SBP before the last CoQ10 injection was $124 \pm 9$ vs $137 \pm 7 \mathrm{~mm} \mathrm{Hg}$ in the control $(\mathrm{p}<0.05)$, DBP was $101 \pm 9$ vs $111 \pm 11 \mathrm{mmHg}$ in the control $(\mathrm{p}=0.068)$, and HR was $414 \pm 16$ vs $440 \pm 19 \mathrm{bpm}$ in the control $(\mathrm{p}<0.05)$. The RBC concentrations of ATP before the last CoQ10 injection was $1.97 \pm$ 0.80 vs $1.13 \pm 0.60 \mathrm{mM}$ in the control $(\mathrm{p}<0.05)$. There was no significant difference between the biomarker data obtained before and those after the last injection ( $\mathrm{p}>0.05$ by paired t-test)

Conclusion: CoQ10 decreased BP and HR and increased RBC concentrations of ATP after multiple injections to normotensive rats

Supported in part by Dalhousie Pharmacy Endowment Foundation and Dalhousie Faculty of Health Profession Research Development Grant.

\section{Drug Delivery \& Pharmaceutical Technology}

\section{Efficacy of an Oral and Tropically Stable Lipid-based Formulation of Amphotericin B (iCo-010) in an Experimental Mouse Model of Systemic Candidiasis}

$\underline{\text { Riley Walsh }}{ }^{1,2}$, Olena Sivak $^{2}$, Fady Ibrahim $^{2}$ and Kishor M. Wasan ${ }^{2}$

1 School of Health Sciences, British Columbia Institute of Technology, Burnaby, BC; ${ }^{2}$ Faculty of Pharmaceutical Sciences, University of British Columbia, Vancouver, BC, Canada

Introduction: Amphotericin B $(\mathrm{AmB})$ is a broadspectrum antifungal and antiparasitic agent used to treat invasive fungal infections. The use of $\mathrm{AmB}$ is limited by its nephrotoxicity and acute side affects due to intravenous administration. An oral and tropically stable (iCo-010) lipid-based formulation was developed to enhance the oral absorption of AmB.

Purpose: Investigate the efficacy of a tropically stable, oral lipid based formulation of Amphotericin B (iCo-010) in a mouse model of systemic candidiasis.

Methods: The mice were infected with $1 \times 10^{8}$ CFU's of Candida albicans ATCC 18804 strain by tail vein injection after which the infection was left to develop for three days. The treatment was then started and each mouse was assigned to the following groups: no treatment (control) and iCo010 at 5,10 and $20 \mathrm{mg} / \mathrm{kg}$ administered via oral gavage once daily (QD) for five consecutive days. After 7 days recovery post treatment the animals were sacrificed and the concentration of $\mathrm{AmB}$ and remaining fungal burden (in colony forming units (CFUs)) were assessed within the kidney, liver, spleen, heart, lungs and brain.

Results: The infection was relatively low $(\sim 60-100$ $\mathrm{CFU} / 1 \mathrm{ml}$ of tissue homogenate) in the liver, lungs and heart, however the infection was relatively high (70 $000 \mathrm{CFU} / 1 \mathrm{ml}$ of tissue homogenate) in the kidney tissues for the control group. The fungal burden in the tissues was lowered by $69-96 \%$ in the treatment groups when compared to the control group. The highest concentrations of $\mathrm{AmB}$ were recovered in the kidneys and the spleen.

Conclusion: Oral administration of iCo-010 once 
daily for five days is an effective treatment for systemic candidiasis in the mouse model.

Funding: CIHR and iCo Therapeutics Inc.

Acknowledgement: Riley Walsh received the National Summer Student Research Program Awards to Present Research Findings sponsored by GlaxoSmithKline Inc.

\section{Investigating the Intraluminal Processing of Arylimidamide DB1960 in a Nanoparticle Lipid Formulation using an in vitro Lipolysis Model}

Carly Wong ${ }^{1}$, Olena Sivak $^{2}$, Fady Ibrahim $^{2}$ and Kishor M. Wasan $^{2}$

${ }^{1}$ Faculty of Science, University of British Columbia, Vancouver, BC, Canada; ${ }^{2}$ Faculty of Pharmaceutical Sciences, University of British Columbia, Vancouver, BC, Canada

Introduction: DB1960 is a mesylate salt arylimidamide (AIA) and is a member of the aromatic diamidines group displaying broadspectrum anti-parasitic activity. DB1960 demonstrated high in vitro efficacy but poor in vivo efficacy against visceral leishmaniasis, a parasitic disease. A nanoparticle lipid formulation of DB1960 was developed to increase its intestinal lymphatic uptake in order to target macrophages, the target cells of Leishmania.

Purpose: To assess the intraluminal processing of the arylimidamide DB1960 in a lipid formulation of Gelucire ${ }^{\circledR} 44 / 14$ and peanut oil (1:1) using an in vitro lipolysis model.

Methods: DB1960 in suspension $(5 \mathrm{mg}, 0.5 \mathrm{ml})$ or in the lipid formulation $(5 \mathrm{mg} / 0.5 \mathrm{ml}, 10 \mathrm{mg} / 1 \mathrm{ml}$, $15 \mathrm{mg} / 1.5 \mathrm{ml}$ ) was added to lipolysis digestion medium composed of $50 \mathrm{mM}$ tris maleate, $150 \mathrm{mM}$ $\mathrm{NaCl}, 5 \mathrm{mM} \mathrm{CaCl} 2,5 \mathrm{mM} \mathrm{Na}$ taurocholate, and $1.25 \mathrm{mM}$ lecithin, which simulated fasted state conditions in the intestine. The medium was maintained at $\mathrm{pH} 6.8$ by a $\mathrm{pH}$ stat titrator and at a temperature of $37^{\circ} \mathrm{C}$. Lipolysis was initiated by the addition of pancreatic lipase/co-lipase to the medium. Lipolysis was considered completed when the rate of $\mathrm{NaOH}$ addition fell to $0.01 \mathrm{ml} / \mathrm{min}$ over one minute. The medium was ultracentrifuged and separated into aqueous and sediment phases, with no oil phase observed due to complete lipid digestion. The percent recovery of DB1960 in each phase was determined using HPLC. The limit of quantification of DB1960 was $4 \mu \mathrm{g} / \mathrm{ml}$.
Results: The lipid formulation led to a significant reduction in the aqueous solubility of DB1960 compared to the control. An increase in the duration of lipolysis with increased doses of lipid formulation was observed, due to an increased volume of lipids added to the lipolysis medium. An increased volume of $\mathrm{NaOH}$ titrated with increased doses of lipid formulation was due to an increased amount of fatty acids liberated by the lipase enzyme from the lipids in the medium.

Conclusion: The aqueous solubility of DB1960 was reduced in the lipid formulation, and more of the drug was distributed to the sediment phase. Modification of the formulation is needed to enhance the lymphatic uptake of DB1960. Different compositions of the DB1960 lipid formulation are currently being investigated though in vitro studies, and one lipid formulation has been tested in an animal model of leishmaniasis.

Acknowledgements: Funding for this study was provided by CIHR, NGDI-UBC, and the Bill \& Melinda Gates Foundation.

\section{Effect of Human Polynucleotide Kinase/Phosphatase Inhibitor on Survival of Acute Myeloid Leukemia Cells Bearing the Leukemic Stem Cell Phenotype $\mathrm{CD123}^{+} / \mathrm{CD}^{-}{ }^{-}$Following Exposure to External Gamma Irradiation or Auger Electron Radioimmunotherapy}

Arman Zereshkian, Zhongli Cai, Jeffrey V. Leyton, Dane Bergstrom and Raymond M. Reilly Department of Pharmaceutical Sciences, Leslie Dan Faulty of Pharmacy, University of Toronto, Ontario, Canada

Objective: CD123 is an attractive cell-surface target for the eradication of leukemic stem cells (LSC) by radioimmunotherapy (RIT). LSCs are believed to initiate and propagate acute myeloid leukemia (AML) ultimately causing relapse in patients. Our lab has developed a radioimmunoconjugate (RIC) that uses low energy Auger electrons emitted by indium-111 ( $\left.{ }^{111} \mathrm{In}\right)$. However, LSCs express low numbers of CD123 epitopes and, as a result, specific killing via CD123 may be limited. We report for the first time a potential role for a human polynucleotide kinase/phosphatase inhibitor (hPKNP) A12B4C3 as a radiosensitizer of AML cells exposed to either external gamma irradiation (EGI) or Auger-emitting radioimmunotherapy (RIT). 
Methods: The CD123-targeted monoclonal antibody (mAb) 7G3, as well as isotype-matched control BM4 was modified with DTPA for chelating ${ }^{111} \mathrm{In}$, and a nuclear localization sequence (NLS) peptide to route ${ }^{111} \mathrm{In}$ to the nucleus. In vitro cytotoxicity of $\mathrm{CD} 123^{+}$AML cells was determined by clonogenic survival (CS) assays. Cells were treated with ${ }^{111}$ In-labeled or unlabeled NLS-7G3 or NLS-BM4 (16-266nM) to determine the optimal condition under which CS of AML cells is CD123 specific. Non-specific cytotoxicity to AML cells exposed to varying concentrations $(5-75 \mu \mathrm{M})$ of A12B4C3 was determined. CS with varying doses (0.25-5Gy) of EGI or ${ }^{111}$ In-NLS-7G3 (16-66nM) with and without $25 \mu \mathrm{M}$ of A12B4C was investigated. DNA damage of AML cells exposed to varying doses (0.5-1.5Gy) of EGI or $33 \mathrm{nM}$ of ${ }^{111} \mathrm{In}$ NLS-7G3 with and without A12B4C 3 was assessed by confocal microscopy and quantified by counting $\gamma-\mathrm{H} 2 \mathrm{AX}$ foci.

Results: There was a concentration-dependent effect on CS of AML cells treated with ${ }^{111}$ In-NLS-7G3 at $66-266 \mathrm{nM}$. This significantly $(\mathrm{p}<0.05)$ reduced CS compared to control. However, ${ }^{111}$ In-NLS-BM4 also significantly $(\mathrm{p}<0.05)$ reduced $\mathrm{CS}$ at $266 \mathrm{nM}$ and 133 $\mathrm{nM}$ compared to control, demonstrating potential non-specific radiotoxicity (NSR). NSR was not observed at $66 \mathrm{nM}$ of ${ }^{111}$ In-NLS-BM4 ( $\left.>0.05\right)$, demonstrating that ${ }^{111}$ In-NLS-7G3 at $66 \mathrm{nM}$ significantly reduces $\mathrm{CS}$ in a CD123-specific manner. The addition of $\mathrm{A} 12 \mathrm{~B} 4 \mathrm{C} 3$ to low doses of EGI $(0.25-0.5 \mathrm{~Gy})$ significantly decreased $(\mathrm{p}<0.05)$ CS compared to AML cells treated with EGI alone. AML cells treated with a non-cytotoxic concentration $(33 \mathrm{nM})$ of ${ }^{111}$ In-NLS-7G3 in combination with $\mathrm{A} 12 \mathrm{~B} 4 \mathrm{C} 3$ showed significant decrease in CS $(\mathrm{p}<0.05)$ comparable to using twice the concentration of RIC alone. Furthermore, cells treated with low doses of EGI or low concentrations of RIC in combination with A12B4C3 significantly increased DNA double strand breaks $(\mathrm{p}<0.05)$ as compared to treatment without radiosensitizer.

Conclusions: A12B4C 3 radiosensitizes AML cells to EGI at low doses and radiosensitizes AML cells in combination with RIT at otherwise non-cytotoxic concentrations, producing an overall cytotoxic effect comparable to higher concentrations of RIC alone. This drug may enhance the cytotoxicity of the RIC, thereby obviating the issue of the relatively low CD123 expression level of the LSC subpopulation, and enabling the use of lower concentrations of RIT, potentially reducing non-specific toxicity to normal cells.
Supported by a grant from Canadian Institutes of Health Research.

Acknowledgement: Arman Zereshkian received the National Summer Student Research Program Awards to Present Research Findings sponsored by GlaxoSmithKline Inc.

\section{Docetaxel-Loaded Polymeric Nanoparticles of PLGA for Sustained Release Intravenous Application: Preparation and In Vitro Characterization}

Pedram Rafiei and Azita Haddadi

College of Pharmacy and Nutrition, University of Saskatchewan, Saskatoon, Saskatchewan, Canada

Purpose: Docetaxel is a highly potent anticancer agent being used in a wide spectrum of cancer treatment regimens. However, there are serious matters of concern regarding docetaxel's pharmacokinetics being related to the drug and conventional formulation. Poly (lactide-coglycolide) (PLGA) is a non-toxic polymer with biocompatible/biodegradable properties whose application in human has been approved by United States Food and Drug Administration (FDA). Nanoparticles with unique drug delivery characteristics have been achieved with this polymer. Herein, we attempt to prepare and fully characterize docetaxel-loaded PLGA nanoparticles as intravenous (IV) sustained-release delivery vehicles for the anticancer agent. Through this investigation, the fluctuations in systemic drug concentration could be minimized and retained within docetaxel's therapeutic window. Therefore, PLGA nanoparticle formulation could enhance the favourable pharmacokinetics, biofate, and pharmacotherapy of the drug in cancer treatment.

Methods: An emulsion solvent evaporation technique was used to fabricate various drug-loaded PLGA nanoparticle formulations. Poly (ethylene glycol) (PEG) surface-modified PLGA nanoparticles were also prepared through the same technique. Effectiveness of different cryoprotective agents in preserving nanoparticle's properties after freezedrying was investigated. Nanoparticles were then fully characterized in terms of particle size, size distribution, zeta potential, drug loading, entrapment efficiency and in vitro drug release profile. Ultimately, viability tests were performed on cancer cells to determine the cytotoxicity efficiency of drug loaded in nanoparticles compared to the free drug. 
Results: PLGA nanoparticles demonstrated a size range between 100 to $140 \mathrm{~nm}$ with negative zeta potential ranging from -20 to $-30 \mathrm{mV}$. They also exhibited a wide range of drug loading (i.e., $0.043 \%$ to $5.24 \%$ ) and entrapment efficiencies (i.e., $15.3 \%$ to $55.65 \%$ ). The particle size of PLGA-PEG nanoparticles was found to be around $180 \mathrm{~nm}$. The drug loading and encapsulation efficiencies were as high as $0.889 \%$ and $96.1 \%$, respectively for PEGylated nanoparticles. Both type of nanoparticles demonstrated biphasic drug release behaviour, giving rise to a burst release during the first 24 hours followed by a sustained drug liberation. The cell viability tests performed on cancer cell lines indicated that loaded drug in nanoparticles is considerably more cytotoxic compared to free drug.

Conclusion: It can be concluded that obtained nanoparticles possess suitable characteristics to fulfil future objectives of the study (intravenous sustainedrelease long circulating drug carriers).

\section{A Comprehensive Study on the Freeze- Drying Process to Increase the Long-Term Stability of Polymeric, Drug-Loaded Nanoparticles}

Hoda Mohamed Besheir, Soudeh Fakhari Tehrani and Valérie Gaëlle Roullin

Pharmaceutical Nanotechnology Laboratory, Faculté de Pharmacie / School of Pharmacy, Université de Montréal, Montreal QC, Canada

Purpose: To investigate the effects of (i) nature and concentration of the cryoprotective agents and (ii) nanoparticle nature and concentration used during the freeze-drying process on the overall stability of resuspended nanoparticle formulations.

Methods: Polymeric nanoparticles, either based on polysaccharides (chitosan, hyaluronic acid, alginate) or on polyesters (PLGA, PLA-PEG), were prepared by previously reported methods. ${ }^{1}$ They were subsequently purified by dialysis, differential centrifugation, tangential flow filtration or a combination of these methods. They were then characterized for (i) size and size distribution by Dynamic Light Scattering; (ii) zeta potential by Electrophoretic Light Scattering and (iii) Drug Loading Efficiency (DLE) and drug release kinetics over $72 \mathrm{~h}$ in phosphate buffer $\mathrm{pH} 7.4,37^{\circ} \mathrm{C}$.

Purified polymeric nanoparticles were then freezedried in a Modulyod Freeze-dryer (Thermo Electron Corporation, USA). Various concentrations of the following cryoprotective agents were added to the nanoparticle suspensions: trehalose, sucrose, glucose, lactose, low $\mathrm{Mw}$ dextran. They were quickly frozen in liquid nitrogen then submitted to freeze-drying for $48 \mathrm{~h}$. Resuspension of the samples took place stepwise, adding small volumes of water every $30 \mathrm{~min}$ until the initial nanosuspension volumes were recovered. Thereafter, the resuspended nanoparticles were fully characterized in the same way as prior freeze drying.

Results: In order to evaluate the impact of mechanical and freezing stresses imposed on the nanoparticles that could lead to nanoparticle destabilization (aggregation, drug leakage) ${ }^{2}$, all parameters tested were rationalized after / prior freeze drying. A maximum deviation of $5 \%$ was tolerated to consider that the assessed parameter was not affected by the freeze-drying process.

On-going analysis should allow optimizing cryoprotectant characteristics / concentration according to the polymeric nature of the nanoparticles and their initial concentration in the suspension. These data will be compared to previous studies by others ${ }^{3}$, so as to verify the generalization of our findings.

Conclusion: This comprehensive approach aimed at defining the best types and concentrations of cryoprotectant and nanoparticle suspensions which guarantee a rapid reconstitution of the nanoparticle product, in relation with the polymeric nature of the nanoparticles. On-going studies will allow generating useful recommendations for the process ability of drug-loaded nanoparticles.

1. Callewaert $\mathrm{M}$ et al. Etoposide encapsulation in surface-modified poly(lactide-co-glycolide) nanoparticles strongly enhances glioma antitumor efficiency. J Biomed Mater Res A. 2013, 101(5):1319-1327. Courant $\mathrm{T}$ et al. Hydrogels incorporating GdDOTA: towards highly efficient dual T1/T2 MRI contrast agents. Angew Chem Int Ed Engl. 2012, 51(36):9119-9122. Sant $\mathrm{S}$ et al. Effect of porosity on the release kinetics of propafenone-loaded PEG-g-PLA nanoparticles. J Control Release. 2005, 107(2):203-14.

2. Abdelwahed $\mathrm{W}$ et al. Freeze-drying of nanoparticles: formulation, process and storage considerations. Adv Drug Deliv Rev. 2006, 58(15):1686-1713.

3. Date PV et al. Freeze thaw: a simple approach for prediction of optimal cryoprotectant for freeze drying. AAPS PharmSciTech. 2010, 11(1):304-313. 


\section{Chitosan-Based Nanogels in Macrogels aimed at the Controlled Release of Active Substances for the Local Treatment of Defective Cartilage}

Ophélie Gourgas ${ }^{1,}{ }^{2}$, Hoda Mohamed Besheir ${ }^{1}$, Xavier Banquy $^{2}$ and Valérie Gaëlle Roullin ${ }^{1}$

${ }^{1}$ Pharmaceutical Nanotechnology Laboratory and ${ }^{2}$ Canada Reasearch Chair in Bio-inspired Materials and Surfaces, Faculté de Pharmacie / School of Pharmacy, Université de Montréal, Montreal QC, Canada

Purpose: The intra-articular injection of medicinal gels for patients with joint diseases, such as osteoarthritis or rheumatoid arthritis, could overcome various problems encountered with current treatments. However, these gels do not yet adequately guarantee availability of the active substances because they are quickly destroyed.

The hypothesis of this study was that (i) the encapsulation of model molecule in chitosan-based nanogels would allow a modulated drug release and that (ii) their subsequent incorporation in model acrylamide macrogels would be possible and were investigated for their application in the local treatment of defective cartilage.

Methods: Ionic gelation process was used for the preparation of chitosan-based nanogels, by electrostatic interactions of cationic chitosan (CS) with polyanions (TPP, hyaluronic acid) in water ${ }^{1}$. The loading of the negatively-charged sulforhodamine B (the chosen model molecule) was obtained by adding increasing concentration of the molecule to the anionic phase. Particle size, size distribution (PdI) and zeta potential of the CS-based nanogels were measured using a Zetasizer NanoZS equipment (Malvern instruments). The encapsulation efficiency (EE) and drug loading efficiency (DLE) were determined by spectrofluorimetry. Release kinetics of the sulforhodamine was performed according to appropriate USP protocols. Acrylamide-based gels were synthesized by photopolymerization, using acrylamide crosslinking with bis-acrylamide ${ }^{2}$. Sulforhodamine-loaded nanogels were incorporated in macrogels by simply diluting nanoparticle suspensions in the reactive medium of macrogel synthesis. The resulting gels were characterized by measuring the elastic modulus, assessing the homogeneity of nanogel distributions, the swelling rate and the sulforhodamine release kinetics.

Results: Chitosan-based nanogels had a particle size of $210 \pm 10 \mathrm{~nm}$, a PdI of $0.23 \pm 0.05$ and a zeta potential of $55.4 \pm 3.6 \mathrm{mV}$. EE\% and DLE\% were optimized to reach values of $92 \pm 0.2 \%$ and $6.1 \pm$ $0.1 \%$, respectively. Incorporation of nanogel particles in macrogels allowed modulating the release kinetics of sulforhodamine. Moreover, the release kinetics also showed a strong dependence on the crosslinking density of the macrogels, the nanogel concentration and the $\mathrm{pH}$. Further investigations should provide information about the mechanical properties of the nanogel-loaded macrogels.

Conclusion: An original approach to obtain controlled-release macrogels was established. This strategy allowed the prolonged release of a model molecule and should be successfully extended to the encapsulation of fragile protein- or peptide-based therapeutic gels for the local treatment of defective cartilage.

1. Courant $\mathrm{T}$ et al. Hydrogels incorporating GdDOTA: towards highly efficient dual T1/T2 MRI contrast Fagents. Angew Chem Int Ed Engl. 2012, 51(36):9119-9122.

2. Leach JB et al. Photocrosslinked hyaluronic acid hydrogels: natural, biodegradable tissue engineering scaffolds. Biotechnol Bioeng. 2003, 82:578-589.

\section{Fabrication and Characterization of a Segmented Reservoir Intravaginal Ring Device for Controlled Release of Hydroxychloroquine}

Amanda Li, Yufei Chen, Yannick Traore, and Emmanuel A. Ho

Laboratory for Drug Delivery and Biomaterials, Faculty of Pharmacy, University of Manitoba, Winnipeg, Manitoba, Canada

Purpose: Studies have demonstrated that immune quiescence (IQ; baseline state of decreased T-cell immune activity) may protect against HIV-1 infection. The goal of this study was to develop and characterize a novel segmented reservoir IVR drug delivery system for the controlled, sustained $(>14$ days) release of hydroxychloroquine (HCQ) to induce the IQ state within the female genital tract. HCQ is an immunomodulatory drug with antiinflammatory and anti-HIV activity.

Method: The polyether urethane reservoir IVR segments were fabricated by hot-melt injection molding. The target cross-sectional diameter of the segment was $25 \mathrm{~mm}$ with a wall thickness of $7 \mathrm{~mm}$. 
IVR segments were loaded with either $4 \mathrm{mg}$ HCQ alone or $4 \mathrm{mg}$ HCQ mixed with a rate-controlling excipient $(1: 1 \mathrm{wt} / \mathrm{wt})$. The ends were sealed with non-water permeable silicone. Release studies were performed at $37^{\circ} \mathrm{C}$ in $5 \mathrm{~mL}$ of $25 \mathrm{mM}$ sodium acetate buffer ( $\mathrm{pH} 4$ ) for 2 weeks. Released HCQ was quantitated using a reversed-phase high performance liquid chromatography method. In vitro cytotoxicity studies were evaluated in the vaginal epithelial cell line Vk2/E6E7 and ectocervical epithelial cell line Ect1/E6E7 using drug-free IVR segments.

Results: IVR segments loaded with HCQ alone showed a slight burst release during the first $48 \mathrm{hr}$ $(391 \pm 29.48 \mu \mathrm{g} ; 9.78 \pm 0.731 \%$ and $491.75 \pm 84.32$ $\mu \mathrm{g} ; 12.29 \pm 2.11 \%$ on day 1 and day 2 , respectively), followed by decreasing release rate of HCQ. IVR segments loaded with HCQ and the rate-controlling excipient demonstrated a nearly zero-order release profile. The average release rate observed was $195.59 \pm 24.96 \mu \mathrm{g}(4.67 \pm 0.59 \%)$ per day for 14 days. No cellular cytoxicity was observed when both vaginal and ectocervial epithelial cells were incubated in the presence of drug-free IVR segments.

Conclusions: Our research group is the first to fabricate a segmented reservoir IVR that can provide controlled, sustained release of HCQ over 14 days. This IVR system is non-cytotoxic and may be a suitable platform for the prevention of HIV transmission.

Acknowledgement: Amanda $\mathrm{Li}$ received the National Summer Student Research Program Awards to Present Research Findings sponsored by GlaxoSmithKline Inc.

\section{Using Peptides to Synthesize Cancer Targeting Compounds}

Howe-Ming $\mathrm{Yu}$, Yogita Raghuwanshi, Kamaljit Kaur

Faculty of Pharmacy and Pharmaceutical Sciences, University of Alberta, Edmonton, Alberta, Canada

Purpose: In order to minimize side effects of chemotherapy, cancer-targeting peptides have been used to selectively deliver chemotherapeutic agents to cancer cells. A previously developed 10-mer peptide, 18-4 (WxEAAYQrFL, where $\mathrm{x}$ is $\mathrm{D}$ norleucine), was found to have high selectivity and stability but slight toxicity effects. A novel analogue to this peptide is developed to have similar selectivity and stability but lower toxicity, where a $\mathrm{D}$-arginine is replaced with an L-lysine (WxEAAYQKFL, 18-4-2).

Methods: This peptide was manually synthesized and coupled to fluorescein isothiocyanate (FITC) through a $\beta$-Ala linker. After purification by reverse phase high performance liquid chromatography and characterization by matrix-assisted laser desorption/ionization time of flight mass spectrometry, $\quad 10^{-4} \mathrm{M} \quad$ FITC- $\beta$-Ala-18-4-2 was incubated with MDA-MB-231 and MDA-MB-435 breast cancer cells for 30 minutes. Cells were then collected and acquired using flow cytometry.

Results: Uptake by MDA-MB-231 cells showed a 1.1 fold increase compared to untreated cells, while MDA-MB-435 cells showed a 1.4 fold increase.

Conclusions: The 18-4-2 analogue shows affinity for breast cancer cell lines MDA-MB-231 and MDA-MB-435, and could potentially be used as a cancer-targeting peptide. Further investigation towards 18-4-2 uptake in other tumorigenic and nontumorigenic cell lines and its stability will be carried out.

(Presented at University of Alberta Faculty of Pharmacy and Pharmaceutical Sciences Centennial Research Day, March 7, 2014, published in Centennial Research Day Book of Abstracts).

Acknowledgement: Howe-Ming $\mathrm{Yu}$ received the National Summer Student Research Program Awards to Present Research Findings sponsored by GlaxoSmithKline Inc.

\section{NMR and Fluorescence Studies of pH- sensitive Liposomes Based on a Molecular Switch}

Warren Viricel, Amira Mbarek and Jeanne Leblond Faculty of Pharmacy, Université de Montréal, Montréal, QC, Canada

Purpose: Even if numerous clinical trials in gene therapy are currently engaged and provide encouraging results, scientists are still trying to improve safety and efficiency of the carriers designed to encapsulate, protect and deliver the therapeutic nucleic acid into the cells. Cationic liposomes are safe non-viral vectors which composition can be tuned to provide specific targeting properties. For instance, cytosolic delivery of siRNA can be enhanced by pH-sensitive lipids, which take advantage of the $\mathrm{pH}$ drop encountered in the endosome to provoke endosomal membrane 
disruption. In this work we report the preparation and characterisation of $\mathrm{pH}$-sensitive liposomes, incorporating a series of original lipids based on a molecular switch. The lipids are designed to change their conformation upon $\mathrm{pH}$ drop and destabilize the liposome and endosome membranes in acidic medium. The conformational change of lipids was evaluated by ${ }^{1} \mathrm{H}$ NMR titration, and the $\mathrm{pH}$-triggered release was monitored by fluorescence.

Methods: A series of lipids, presenting various headgroups and aliphatic anchors, was synthesized as previously reported. The change of conformation upon acidification was evaluated in protic organic medium by ${ }^{1} \mathrm{H}-\mathrm{NMR}$ titrations. Liposome preparation was done by rehydration of a lipidic film (pH-sensitive lipid/POPC/DSPE-PEG 2000 50:45:5) with a $30 \mathrm{mM}$ solution of sulforhodamine $B$ buffered at $\mathrm{pH}=7.4$. After 10 freeze-thawing cycles and manual extrusion, liposomes were purified by size exclusion chromatography. Liposomes were characterized regarding their size and by DLS-ELS and quantification of the batch was done using the Bartlett assay. To study the $\mathrm{pH}$ dependence of the liposome leakage, a small aliquot of the liposome preparation was incubated in a buffer, and the fluorescent intensity of the leaking sulforhodamine $\mathrm{B}$ was monitored.

Results: ${ }^{1} \mathrm{H}-\mathrm{NMR}$ titrations and NOESY experiments showed that our synthetic lipids are able to change their conformation in an organic protic medium upon acidification. They can successfully form stable liposomes with POPC co-lipid exhibiting diameter smaller than $300 \mathrm{~nm}$. The sulforhodamine B leakage assay showed us that our liposomes are stable at $\mathrm{pH} 7.4$, but are releasing 30\% of their content at $\mathrm{pH}$ values below 4 .

Conclusion: We successfully reported the elaboration of $\mathrm{pH}$-sensitive liposomes, incorporating a synthetic $\mathrm{pH}$-sensitive lipid we designed. The NMR experiments showed us that our lipids can change their conformation upon acidification, and the fluorescence experiments have revealed that our liposomes are releasing their content at acidic $\mathrm{pH}$.

\section{In vitro Comparison of the Efficiency of Levofloxacin-Loaded Polymeric Nanoparticles Versus Liposomes to Kill Pseudomonas Aeruginosa Developing in Cystic Fibrosis Patients}

Juliana Campos Del'Orto, Kevin Plourde, Soudeh F. Tehrani, Valérie Gaëlle Roullin, Patrice Hildgen, Jeanne Leblond Chain

Axe Formulation et Analyse du Médicament, Faculté de Pharmacie / Faculty of Pharmacy, Université de Montréal, Montreal QC, Canada

Purpose: Although Cystic Fibrosis (CF) is a multisystemic disease, the lung phase severely impacts on the patient's lifespan. Indeed, CF patients present an increased production of high viscid mucus which favors the attachment of bacteria and impairs the diffusion of therapeutics. As a consequence, subinhibitory drug concentration leads to amplified bacterial resistance, production of biofilms and treatment failure. In this study, we propose to encapsulate levofloxacin into nanocarriers to improve the antibiotic efficiency in Pseudomonas aeruginosa isolated from cystic fibrosis patients.

This work aimed at (i) synthesizing and characterizing mucus-penetrating PEG-g-PLA nanoparticles and PEGylated liposomes, both loaded with the antibiotic levofloxacin; (ii) comparing the efficacy of free and encapsulated levofloxacin on $P$. Aeruginosa, either in its planktonic or its biofilm form.

Methods: Poly(ethylene glycol)-g-poly(lactic acid) (PEG-g-PLA) was synthesized as previously published $^{1}$. Levofloxacin-loaded and unloaded PLAg-PEG nanoparticles (NPs) were produced by nanoprecipitation $^{2}$ Levofloxacin-loaded and unloaded liposomes of DSPC/Chol/DSPE-PEG2000 (55:40:5) were produced using the ammonium sulfate gradient method ${ }^{3,4}$.

Mean hydrodynamic diameters, size distributions and zeta potentials of both formulations were characterized by DLS-ELS. Levofloxacin encapsulation was assessed by HPLC analysis. The Broth Microdilution Test in mucoid, non mucoid isolated from CF patients and ATCC 57853 strains of $P$. aeruginosas was performed over 48 hours on the planktonic and biofilm forms of the bacteria. The antibacterial efficiency was assessed by the Minimal Inhibitory Concentration (MIC) of levofloxacinloaded NPs/liposomes as compared to free levofloxacin (positive control) and unloaded NPs/liposomes (negative controls). 
Results: Both PEG-g-PLA and PEgylated liposomes were successfully prepared and resulted in nanocarriers whose characteristics are compatible with mucus-penetrating particles and pulmonary administration (mean size: $116 \pm 17$ and $123 \pm 1 \mathrm{~nm}$, respectively). Both systems were able to encapsulate levofloxacin: liposomes displayed a higher loading efficiency (15\%), whereas polymeric nanoparticles resulted in higher encapsulation efficiency (39\%).

Mucoid and non mucoid strains of $P$. Aeruginosa were characterized by MIC values of $1 \mu \mathrm{g} \cdot \mathrm{mL}^{-1}$ and $64 \mu \mathrm{g} \cdot \mathrm{mL}^{-1}$ for free levofloxacin, demonstrating that levofloxacin was able to efficiently target $P$. Aeruginosa, the microorganism related with the increase of mortality and morbidity in CF patients ${ }^{5}$. Moreover, encapsulated levofloxacin was able to efficiently neutralize the planktonic form of $P$. Aeruginosa.

Conclusions: Levofloxacin encapsulation was performed in two PEGylated nanoformulations, with final characteristics addressing well the challenges of the pulmonary route of administration in $\mathrm{CF}$ patients. Furthermore, on-going microbiological assays on $P$. Aeruginosa directly retrieved from $\mathrm{CF}$ patients' mucus should demonstrate the therapeutic relevance of this strategy.

1. Nadeau, V., et al., Synthesis of new versatile functionalized polyesters for biomedical applications. Polymer, 2005. 46(25):p.11263-11272. 2. Callewaert, M., et al., Etoposide encapsulation in surface-modified poly(lactide-co-glycolide) nanoparticles strongly enhances glioma antitumor efficiency. J. Biomed. Mater. Res. A, 2013. 101A(5): p. 1319-1327.

3. Zhang, X, et al. Targeted delivery of levofloxacinliposomes for the treatment of pulmonary inflammation. Journal of Drug Targeting. 2009; 17(5):399-407.

4. Haran, G, et al. Transmembrane ammonium sulfate gradients in liposomes produce efficient and stable entrapment of amphipathic weak bases. Biochimica et Biophysica Acta 1993;1151(2):20115.

5. Canada:, C.F., Canadian Cystic Fibrosis Registry 2011 Annual Report. Cystic Fibrosis Canada, 2011.

\section{Microdialysis Procedure to Investigate the Extent of Neutralization of Palliative Calcium Gluconate Following Ophthalmic Hydrofluoric Acid Exposure}

Navpreet Pandher, Murali Mohan Bommana, Emilio Squillante III, Virender Kumar

Department of Pharmaceutical Sciences, College of Pharmacy and Allied Health Professions, St. John's University, Queens, NY

Purpose: The performance and validity of the microdialysis system in a time based calibration of the fluoride ion permeation, inside the eye following HF burn was tested. The HF diffusion was measured in terms of the fluoride content recovered in the microdialysate samples after spillage of $0.15 \mathrm{ml}$ of acid over the cornea in the plucked sheep eyes with or without neutralization with calcium gluconate formulations. The in vitro and in situ probe calibration and integrity test for assessing the diffusion of acid was performed.

Methods: The stability of 10 BAS microdialysis probes was tested with $0.05 \mathrm{M} \mathrm{HF}$ by calculating the in vitro probe recovery. The effect of flow rate variation on recovery of fluoride ions was evaluated by performing an in vitro study for $0.01 \mathrm{M} \mathrm{HF}$, with a constant sample volume of $40 \mu 1$ at flow rates of $0.5,1.0,2.0$ and $4.0 \mu \mathrm{l} / \mathrm{min}$. The in situ probe calibration was performed on the aqueous and vitreous humor samples of the sheep eye, which could provide the baseline fluoride concentrations required for the calcium gluconate therapy. Two different formulations, solution $(2.5 \%$ and $5.0 \% \mathrm{w} / \mathrm{v}$ calcium gluconate) and the 3\% HPMC gel containing $2.5 \% \mathrm{w} / \mathrm{v}$ calcium gluconate were subjected to neutralization potential study. A comparison study of HF neutralization with the calcium gluconate gel vs. Methocel control formulation was performed to eliminate any contribution from the gel component.

Results: The BAS probe was accurate and stable with a CV of $0.44-2.45 \%$. A flow rate of $4.0 \mu 1 / \mathrm{min}$ can be used for fluoride permeation study. The results showed that the MD probe is capable of dialyzing the HF solution without interacting with the probe. The in situ testing showed that the HPMC based calcium gluconate formulation showed longer residence time compared to the solution form.

Conclusions: The microdialysis technique could be used to ascertain the relative movement of fluoride ions penetrating in the HF-burnt eyes. By increasing the amount of calcium ions available on eye surface, 
increases the free acid neutralization; the gel preparation being better than the solution of same concentration. It can be concluded that only gel therapy provides necessary counter ion to neutralize the destructive power of fluoride ions peri- and intraocularly presumably by offering the necessary "sink" conditions that can draw fluoride from the affected eye and because of reduced clearance from ocular surface.

\section{Preparation of Micelle-forming $\mathrm{ABC}$ Tri- Block Copolymers as Nano-reservoirs for Controlled Drug Release}

Hoda Soleymani Abyaneh, Mohammad Reza Vakili, Fanglin Zhan, Afsaneh Lavasanifar

Faculty of Pharmacy and Pharmaceutical Sciences, University of Alberta, Edmonton, AB, Canada

Purpose: The objectives of this study was to: 1) prepare $\mathrm{ABC}$ triblock copolymers and their micellar counterparts; and 2) investigate the effect of the three layered micellar structure on drug encapsulation and release, making comparison with two layered core/shell micelles prepared from diblock copolymers.

Methods: $\mathrm{AB}$ diblock copolymers consisting of methoxy poly(ethylene oxide) (MePEO) (as the A block) and poly(lactide)s (PLA)s of different stereochemistry (as the B block) as well as ABC triblock copolymers consisting of PEO (as the A block); poly(lactic acid)s with different stereochemistries (as the B block) and $\operatorname{poly}(\alpha-$ benzylcarboxylate- $\varepsilon$-caprolactone) (PBCL) (as the $\mathrm{C}$ block) were synthesized by bulk ing opening polymerization using stannous octoate as catalyst. Di- and triblock copolymers were self-assembled to polymeric micelles. Polymers and/or micelles were characterized for their chemical structure by ${ }^{1} \mathrm{H}$ NMR, optical rotation by polarimetry, thermal properties by differential scanning calorimetry, and sizes by dynamic light scattering. Encapsulation and in vitro release of a model hydrophobic drug, nimodipine, from the self-assembled structures were assessed using UV spectroscopy to measure drug levels.

Results: Successful synthesis of di-and triblock copolymers was confirmed by ${ }^{1} \mathrm{H}$ NMR. The sizes for loaded and empty micelles showed a narrow mono-disperse size distribution within an ideal range of 50-130 nm. Encapsulation of nimodipine reached more than $50 \%$ efficiency in tiblock copolymer micellar structures in contrast to $30 \%$ efficiency in diblock copolymers. A significant reduction in the burst release of nimodipine incorporated in triblock copolymeric micelles was observed at initial time points compared to diblock copolymer micelles in vitro.

Conclusions: Synthesis of ABC block copolymers based on PEO-PLA-PBCL is a feasible approach. Triblock copolymer micelles were successful in improving the drug loading and release profile compared to diblock copolymer micelles, preventing the initial burst release while achieving a significantly higher drug loaded levels.

\section{Investigating the Structure of $\mathrm{pH}$-sensitive Lipids Based on a Molecular Switch}

Amira Mbarek, Warren Viricel, Aline Mesnier and Jeanne Leblond Chain

Faculty of Pharmacy, University of Montreal, QC, Canada

Purpose: The success of gene therapy is related to the development of gene vectors able to overcome several biological barriers to accomplish nucleic acid delivery. Among these barriers, endosome remains a major obstacle that compromises successful nucleic acid delivery. As an attempt to avoid the endosomal degradation, we aim to develop $\mathrm{pH}$-sensitive liposomes as vectors for nucleic acids. $\mathrm{pH}$-sensitive lipids were designed based on a molecular switch composed of anisole-pyridine-anisole tricyclic unit. Upon protonation, the pyridine unit induces a conformational change. To investigate if this change was maintained within a lipid structure, we modified the aliphatic chain length and the hydrophilic head of the lipid.

Methods: The assembly of the tweezer unit was accomplished by Suzuki coupling between methyl 2,6-dibromoisonicotinate and 5-bromo-2methoxyphenylboronic acid. Aliphatic chains $\left(\mathrm{R}_{2}\right)$ were introduced thanks to Sonogashira coupling conditions. Subsequent ester saponification allowed peptidic coupling with amine derivatives of the headgroups $\left(\mathrm{R}_{1}\right)$.

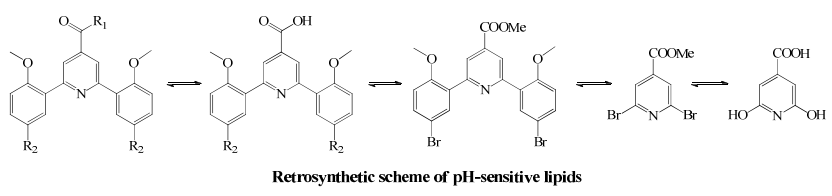

Results: A library of $9 \mathrm{pH}$-sensitive lipids based on 
a molecular switch was synthesised using classical cross-coupling chemistry. Chain lengths were varied from 10 to 16 carbons, in order to tune the hydrophobic interaction between the two aliphatic chains. Various headgroups, from $\mathrm{COOH}$ to cationic amines, were synthesized in order to help hydration and protonation of the lipid.

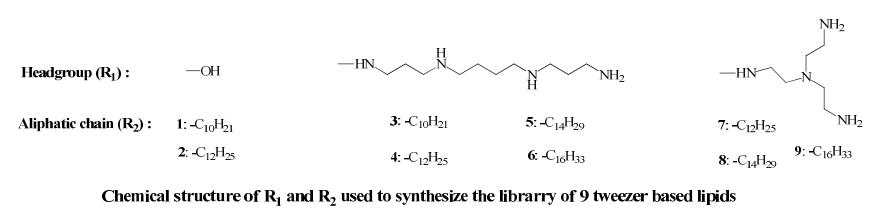

Conclusion: A library of 9 lipids have been synthesized and fully characterized to examine the structure-relationship of $\mathrm{pH}$-sensitive innovative lipids based on a molecular switch. The influence of each structure parameter on the membranedestabilization will be evaluated by solid NMR $\left({ }^{31} \mathrm{P}\right.$ and $\left.{ }^{2} \mathrm{D}\right)$.

\section{Modeling the Absorption of Metformin in Patients with Post Gastric Bypass Surgery}

May Almukainzi, Raimar Löbenberg

Faculty of Pharmacy \& Pharmaceutical Science, University of Alberta, Edmonton, AB, Canada

Purpose: Gastric bypass surgery in obesity shortens the length of the small intestine, which can have a significant impact on drug absorption. Literature reports that the observed drug absorption patterns after gastric bypass surgery are sometimes unexpected. One report states that the absorption of Metformin was higher after gastric bypass surgery. The purpose of this study was to investigate the mechanistic background of the reported data using a physiologically based pharmacokinetic (PBPK) computer model and apply it to patients with post gastric bypass surgery.

Methods: GastroPlusTM 8 (Simulations Plus, Inc.) was used to develop a model that describes the observed absorption of an immediate release (IR) metformin tablet in healthy subjects. The data was taken from a published article that compared the absorption of metformin between a control group and post gastric bypass surgery patients. The fitted model of the control group was then used to predict the drug absorption in post gastric bypass surgery patients by changing the related GI parameters. All assumptions, suggested in the literature, were tested by changing the appropriate parameters in the software.

Results: As theoretically expected, Gastroplus underestimated the absorption of metformin in patients post gastric bypass surgery due to the lesser absorption area. The only way to simulate the observed data was to increase the pore size and pore ratio of the last part of the small intestine. Changing any other parameters failed to predict the observed absorption pattern.

Conclusion: The only change that was able to simulate the observed absorption of metformin in post gastric bypass surgery patient was a change in the gut's paracellular permeability. This indicates that the gut must have undergone an adaption process to compensate for the last of parts of the small intestine. The insights gained by this study can be used to predict the absorption of other drugs that have similar physiochemical properties like metformin. Computer simulations can be used to simulate the impact and mechanistic background of disease or other physiological changes like surgery on drug absorption.

\section{Cyclodextrin-based Nanoparticles Overcome Drug Resistance to Antimelanoma Drug}

Caitlin Strugari, Masoomeh Poorghorban, Deborah Michel and Ildiko Badea

College of Pharmacy and Nutrition, University of Saskatchewan

Purpose: Melanoma, the most aggressive form of skin cancer, has few effective treatment options secondary to a high incidence of chemoresistance. Novel approaches, such as encapsulating the drug in nanoparticles, have the potential to overcome biological barriers and drug resistance. $\beta$-cyclodextrin coupled to gemini cationic surfactants (CDgemini) assembles into nanoparticles that have demonstrated the ability to encapsulate cytotoxic drugs and effectively deliver them into cells. In order to evaluate the ability to overcome drug resistance, A375 melanoma cells were induced to develop chemoresistance to melphalan, an alkylating agent with high resistance rates, and treated with the drug encapsulated in CDgemini nanoparticles.

Methods: A375 melanoma cells were cultured and treated with increasing concentrations of melphalan over seven weeks to induce drug resistance. The resistant cells were treated with IC25 $(20 \mu \mathrm{M})$ and IC50 $(40 \mu \mathrm{M})$ concentrations of melphalan in $1 \%$ 
acidified ethanol, and encapsulated into CDgemini at 1:2 molar ratios. MTT assay was employed to evaluate the cell viability.

Results: Cell viability after naked melphalan treatments at $20 \mu \mathrm{M}$ and $40 \mu \mathrm{M}$ concentrations $(110 \pm 19 \%$ and $91 \pm 18 \%$, respectively) was comparable to untreated cells. The delivery agent, CDgemini at $80 \mu \mathrm{M}$ concentration had no effect on cell viability $(112 \pm 25 \%$ of the untreated cells). Melphalan in CDgemini nanoparticles induced significant cell death, reducing the cell viability to $28 \pm 9 \%$ at $20 \mu \mathrm{M}$ concentration and to $19 \pm 3 \%$ at 40 $\mu \mathrm{M}$ concentration. The resistant cells also demonstrated changes in morphology, like rounded cell membranes and shrinkage or enlargement of the cells.

Conclusion: Cell viability was high for melphalan in acidified ethanol and low for melphalan encapsulated by CDgemini. Therefore, the CDgemini was able to overcome the chemoresistance acquired by the melanoma cells. Further research is needed to determine the pathways for the resistance and the mechanism by which CDgemini nanoparticles overcome resistance.

Acknowledgement: Caitlin Strugari received the National Summer Student Research Program Awards to Present Research Findings sponsored by GlaxoSmithKline Inc.

\section{Development of Nanostructured Hydrogel for Spatial and Temporal Controlled Release of Active Compounds}

\section{Shaker Alsharif, Xavier Banquy}

Université de Montréal, Montréal, QC

Purpose: The research aims to develop and build hydrogels with different compositions as well as variable percentages of crosslinkers with nanoinclusion of prepared and characterized liposomes loaded with active compounds such as proteins and growth factors able to release them in a controlled fashion.

Methodology: Liposomes nanoparticles encapsulating sulforhodamine $\mathrm{B}$, based on phospholipids (Dipalmitoylphosphatidylcholine (DPPC), 1, 2-Dioleoyl-sn-glycero-3-phosphocholine (DOPC), in different ratios were prepared using freeze-thawing method after film hydration using sulforhodamine B followed by extrusion method in order to get 100-200 nm liposomes . Then, liposomes were purified using Size Exclusion
Chromatography method using Sephadex-50. Subsequently, they were characterized for (i) zeta potential, particle size, isoelectric point (IEP), and critical coagulation concentration (CCC) as a function of $\mathrm{pH}$ and liposome composition using Dynamic Light Scattering and Auto-Titrator; (ii) fluorescence to determine the sulforhodamine B release extent from liposomes using Spectrofluorometry. On the other hand, Gel synthesis by photopolymerization took place using $0.1 \%$ of riboflavin as a source of free radical in combination with Tetramethylethylenediamine TEMED and $10 \%$ ammonium persulfate. In the presence of light and oxygen the riboflavin will be converted to its Leuco form, which is active in initiating photopolymerization. Further hydrogel characterizations for studying mechanical properties and for the release of sulforhodamine in the hydrogel as a function of gel composition (crosslinker percentage: $1 \%-15 \%$ ) will be examined. Consequently, liposomes and hydrogel will be mixed and as a function of Liposome concentration and composition as well as Hydrogel composition, release characterization will be performed.

Results: In order to measure the release rate of synthesized liposomes encapsulating Sulforhodamine $\mathrm{B}$ as a marker, fixed testing parameters were adjusted among multiple batches of different liposomal concentrations. Complete liposomal release has been achieved over one week with almost constant controlled release over the days. Further methods and analysis processes should allow reaching higher stability and longer controlled release over longer period of time.

Conclusions: The research comprehensive approach is to address the ideal methods for production of highly stabilized liposomes as well as hydrogel in terms of composition and concentration, which ensure long-term controlled release of medically active compounds. Current studies have allowed creating useful suggestions and solutions to improve the loaded liposomes stability as well as to control the release over time. 


\section{Co-Printing of Flexible Dose Combinations of Levothyroxine and Liothyronine for the Treatment of Hypothryroidism}

\author{
Mustafa Alomari ${ }^{1}$, Joseph Standing ${ }^{2}$, Abdul Basit ${ }^{1}$, \\ Simon Gaisford ${ }^{1}$ \\ ${ }^{1}$ UCL School of Pharmacy, London, UK; ${ }^{2}$ UCL \\ Institute of Child Health, London, UK
}

Purpose: Hypothyroidism affects $2 \%$ of the total Canadian population. Levothyroxine (T4) is the mainstay of the treatment for the condition. About $13 \%$ of patients remain with health complaints despite replacement T4 therapy. Studies in rats have shown that the T4 replacement does not result in normal thyroid hormone levels (Euthyroidism). This was only achieved when T4 was combined with liothyronine (T3). The clinical benefit of T4:T3 combination in humans lacks consensus, but the ratio of two medicines studied varied significantly from $3: 1$ to $20: 1 \quad(\mathrm{~T} 4: \mathrm{T} 3 \mathrm{w} / \mathrm{w})$. Personalised combination of the two medicines may address the variation in patients' responses to the combination therapy. Thermal Inkjet Printing (TIJ) has the potential to combine the two medicines in flexible ratios on edible substrates ad libitum, suiting the need of individual patients.

Methods: Black and Tricolour TIJ HP printer cartridges were rinsed and filled with solutions of levothyroxine and liothyronine respectively. The two cartridges were then mounted on the printer. Experimental y-values were used to control the amount of each medicine deposited on substrates so that each printed area contained a different ratio of T4:T3. Drug content was quantified with HPLCUV.

Results: Therapeutic doses of both medicines were printed. Individual cartridge control was demonstrated enabling flexible control of T4 and T3 deposition by means of a Microsoft Word template. The printed doses of each medicine, controlled by the dictated y-value, exhibited remarkable linear control and high dose precision, satisfying the dose control requirements for each medicine.

Conclusion: TIJ is a versatile technology which may be adapted to tackle some of the challenges of drug delivery and enabling a personalised approach to dosing medicines.

\section{Development of a New Controlled-Release Oxybutynin Tablet Using a Programmable Rupturable Film Coating Approach}

\author{
Miloud Rahmouni and Mohamed Nabil Khalid \\ Biopharmaceutical Production Technology \\ Department, John Abbott College, Sainte-Anne-de- \\ Bellevue, Quebec, Canada
}

Purpose: Oxybutynin hydrochloride $(\mathrm{Ox}-\mathrm{HCl})$ is used to relieve urinary and bladder difficulties, including frequent urination and inability to control urination. The drug product is currently available in the Canadian market under the brand name of Ditropan XL using Alza's osmotic oral controlledrelease system $\left(\mathrm{OROS}^{\mathbb{B}}\right)$. The purpose of the present work is to design and evaluate a new controlledrelease (CR) tablet formulation of Oxybutynin. $\mathrm{HCl}$ using a programmable rupturable film coating. The new delivery system would be an affordable alternative to the expensive OROS $^{\circledR}$ technology.

Method: Tablets containing Oxybutynin. $\mathrm{HCl}$ and Hydroxypropylmethylcellulose (HPMC) were prepared by direct compression using an instrumented tablet press (RoTab $\mathrm{T}$, Luxner, Germany). The tablets were then film coated in a pan coater (LabCoater, Vector, USA) using a mixture of Ethylcellulose (Surelease ${ }^{\mathbb{R}}$ ) and a poreformer (Opadry $\left.{ }^{\circledR}\right)$. A three-factor, two-level, full factorial design was used to investigate the coating level, the percentage of the poreformer, and the concentration of HPMC in the formulation on the lag-time and the drug release rate of Oxybutynin.HCl. Drug release kinetics was evaluated using a USP dissolution apparatus type II at $50 \mathrm{rpm}$ in phosphate buffer $\mathrm{pH} 6.8$.

Results: An increase of the film coating thickness of the tablet has a significant influence on both the lagtime and drug release rate of Oxybutynin. $\mathrm{HCl}$. An increase in the percentage of the poreformer helped in the rupture of the film coat. As the water uptake of the tablet increases, the pressure generated by the axial swelling of the core increases, then induces the rupture of the surrounding film coat. The percentage of HPMC in the formulation has a significant effect on the drug release rate, but it has no effect on the lag-time. Once the surrounding film coat of the tablet is ruptured, the drug release profile of Oxybutynin. $\mathrm{HCl}$ follows quasi-zero order kinetics, which is similar to that of Ditropan XL.

Conclusion: Using a full factorial design, it was possible to achieve the desired lag time and drug release kinetic of Oxybutin. $\mathrm{HCl}$, mimicking the 
OROS $^{\circledR}$ osmotic pump profile. This new drug delivery system represents an affordable platform technology for programmable rupturable film coated tablet.

\section{Effect of Anti-Psoriatic Drugs on Pathological Skin Substitutes Produced by Tissue Engineering}

$\underline{\text { Isabelle Gendreau }}^{1}{ }^{\text {; }}$ René C.-Gaudreault ${ }^{2}$; Roxane Pouliot ${ }^{1,3}$

${ }^{1}$ Centre LOEX de l'Université Laval - Centre de recherche FRSQ du CHU de Québec; ${ }^{2}$ CRCHUQHôpital Saint-François d'Assise, Québec; ${ }^{3}$ Faculté de Pharmacie, Université Laval, Québec, Canada

Purpose: Psoriasis is a skin disorder that is characterized by reddish and whitish plaques. These plaques are the result of a hyperproliferation and an abnormal differentiation of keratinocytes. Up to now, no curative treatment is available to treat the disease, however, there are treatments that can reduce symptoms. The objective of this project was to evaluate the anti-psoriatic potential of a new drug using our psoriatic skin substitute model produced by tissue engineering.

Methods: Healthy and psoriatic skin substitutes were produced using the self-assembly method developed at LOEX. Briefly, the fibroblasts were cultured in the presence of ascorbic acid until they form manipulable sheets. These sheets were superimposed and incubated for seven days to form a new dermal layer. Then, keratinocytes were seeded on the dermal layer to form a new epidermal layer. After seven days, the substitutes were raised to the air-liquid interface. After two weeks of air-liquid interface culture, substitutes were treated with different concentrations of 4-tert-butyl-ethyl-urea (antineoplastic with anti-psoriatic potential) during one or two weeks and compared to two currently used drugs in the treatment of psoriasis, which are methotrexate (antineoplastic) and dexamethasone (corticosteroid). Following these treatments, macroscopic, histological, immunofluorescence and percutaneous absorption analyzes were performed.

Results: Histological and immunofluorescence analyzes of samples treated with 4-tert-butyl-ethylurea have demonstrated different effects on proliferation and differentiation compared to methotrexate and dexamethasone. Moreover, percutaneous absorption results have shown that this new molecule has the ability to diffuse through the skin whereas large amount remains in the epidermis. This result demonstrates that this molecule could possibly be used as a topical treatment for psoriasis. Indeed, psoriatic substitutes treated with methotrexate have shown a decrease of the psoriatic hyperproliferation and a better differentiation of keratinocytes.

Conclusion: These results show that 4-tert-butylethyl-urea provides a positive effect on our psoriatic skin model as well as drugs frequently used to treat psoriasis. This study confirms that our pathological skin model would be a useful tool for pharmaceutical research.

\section{A Novel Therapeutic Textile Imprinted With Dermotex ${ }^{\circledR}$ Econazole-Loaded Microparticles}

Mirza Akram Hossain ${ }^{1}$, Augustine Lalloz ${ }^{1}$, Aicha Benhaddou $^{2}$, Pierre Simard ${ }^{2}$, Karine Théberge ${ }^{2}$, Martine Raymond ${ }^{3}$ and Jeanne Leblond-Chain ${ }^{1}$

${ }^{1}$ Faculty of pharmacy, University of Montreal, Montréal, QC; ${ }^{2}$ Biomod Concepts Inc, Sainte-Julie, QC; ${ }^{3}$ Institution for research in immunology and cancer, University of Montreal, Montréal, QC, Canada

Purpose: A novel therapeutic textile containing econazole-loaded microparticles was developed using the patented Dermotex ${ }^{\circledR}$ technology. This technology consists in the preparation of lipid microparticle formulations of pharmaceutical active compounds followed by a modified print screening technique to deposit the formulation on a textile surface and other flexible medias. This innovative drug delivery system was developed to improve the bioavailability of active molecules and consequently, reduce posology and improve patient compliance. We hypothesized that textile formulation of econazole maintain or even improve pharmaceutical activity over the commercial Pevaryl ${ }^{\circledR}$ formulation. As a first proof of concept, lipid formulation was optimized for econazole encapsulation and printed on textiles; the antifungal activity of resulting textiles was evaluated in vitro on Candida albicans.

Methods: Microparticles were prepared and imprinted on a Laya ${ }^{\mathrm{TM}}$ textile. Content was analyzed on a square $(1 \times 1 \mathrm{~cm})$ of textile, extracted by methanol $(1 \mathrm{~mL})$, sonicated for 30 minutes and heated for 4 hours at $55^{\circ} \mathrm{C}$. After filtration, the econazole concentration was determined using a validated HPLC-UV method (methanol/water gradient, $220 \mathrm{~nm}$ ). Antifungal activity was 
determined on C. albicans strain SC 5314 using the Kirby-Bauer method. Econazole-loaded textiles were compared to commercial cream formulation. The disks were incubated for 18 hours before measuring the inhibition diameter. These tests were repeated independently 3 times with 3 or more identical plates each time.

Results: Econazole textiles presented a mean of 75 $\mu \mathrm{g} / \mathrm{cm}^{2}$ for every batch. In vitro evaluation on $C$. albicans demonstrated similar activity of econazole with Dermotex ${ }^{\circledR}$ textiles as compared to the commercial formulation after 18 hours. The mean diameter inhibition of econazole on textile formulation was $27 \pm 1.8 \mathrm{~mm}$ compared to Pevaryl ${ }^{\circledR}$ formulation, at equal quantity, which was $24.7 \pm 2.5$ $\mathrm{mm}$. We used the calculated ratio of growth inhibition of textile on Pevary $l^{\circledR}$ formulation for each plates. In 21 total repeated experiments, the activity of econazole on textile was $110 \%$ of the activity of commercial formulation, demonstrating its bioequivalence. Finally, once re-plated on a new C. albicans growth, econazole-loaded microparticles on textile still demonstrated inhibition of the fungus with an inhibition efficacy of $20.1 \pm 1.9 \mathrm{~mm}$.

Conclusion: A new textile containing lipidmicroparticles of econazole was developed, and was shown to exhibit a similar antifungal activity as the commercial cream formulation. Further in vivo and in vitro studies are ongoing to demonstrate the pharmaceutical efficacy of imprinted textiles. Release content profile of the drug from the microparticles is also under investigation.

\section{Gene Profiling Characterization of a Psoriatic Skin Model}

Claudia Pouliot-Bérubé ${ }^{1}$; Roxane Pouliot ${ }^{1,2}$

${ }^{1}$ Centre LOEX de l'Université Laval, Génie tissulaire et régénération : LOEX - Centre de recherche FRQS du Centre hospitalier universitaire de Québec, Québec, Canada; ${ }^{2}$ Faculté de Pharmacie, Université Laval, Québec, Canada.

Purpose: Psoriasis is a condition characterized by an altered expression of many genes. The physiopathology is represented by parakeratosis, epidermal hyperplasia, hyperkeratosis, leukocyte infiltration and neovascularization. This condition affects up to $3 \%$ of the world population, and to this day it remains an incurable disease. As it is impossible at present to test anti-psoriatic drugs on many pathological samples (ethical, availability, interpersonal variations problems and so on) drug formulations offered to the psoriatic population are not well appropriate to their actual situation. The need for innovative and effective tools to evaluate new dermopharmaceutical formulations is essential. This project aims to characterize the gene expression pattern of our psoriatic skin model produced by the self-assembly method.

Methods: DNA microarrays analyses were performed in order to determine the level of mRNA expression of lesional or non-lesional psoriatic skin substitutes and compared to healthy substitutes. Pathological and healthy cells were also analysed. Total RNA was isolated from 5 differents psoriatic skin substitutes, after 2 months of culture. Cyanine 3-CTP labeled cRNA targets were prepared, using the Agilent One-Color Microarray-Based Gene Expression Analysis kit. cRNA was incubated on a G4851A SurePrint G3 Human GE $8 \times 60 \mathrm{~K}$ array slide. Slides were then hybridized, washed, and scanned on Agilent SureScan Scanner. Data were analysed using the ArrayStar V4.1 software for scatter plots and generation of the heat maps of selected genes of interest.

Results: The results obtained so far with monolayer cells, as well as psoriatic skin substitutes reveal a large number of deregulated genes between psoriatic and healthy conditions. Moreover, it appeared that different genes were overexpressed or dowregulated depending of the skin condition (lesional vs nonlesional skin substitutes). Indeed, this pathological cutaneous substitute model could lead to a better understanding of basic mechanisms responsible for the development of psoriasis disease.

Conclusion: The advanced features of this work allow us to confirm that the gene analysis of psoriatic skin substitutes is an effective method to clarify the differences noticed in previous macroscopic and histological analyses of substitutes reconstructed from different cell populations.

\section{RNAi-Based Nanomicrobicide for the Prevention of Male-to-Female Transmission of HIV-1}

Sidi Yang, Jijin Gu, and Emmanuel A. Ho Laboratory for Drug Delivery and Biomaterials, Faculty of Pharmacy, University of Manitoba, Winnipeg, Manitoba, Canada

Purpose: The goal of this study is to develop and characterize an intravaginal $\mathrm{CD} 4+$ immune cell 
targeted nanomedicine for the active delivery of small interfering RNA (siRNA) as a strategy to reduce the expression of viral genes or host factors involved in HIV-1 infection. This drug delivery system is designed for intravaginal administration as a potential pre/post-exposure prophylaxis to help women defend against HIV-1.

Methods: siRNA was first condensed by polyethyleneimine (PEI) and then encapsulated into nanoparticles (NPs) by a double-emulsion evaporation method using the biodegradable diblock copolymer, poly(lactic-co-glycolic acid)polyethylene glycol (PLGA-PEG). NPs were conjugated to anti-human anti-CD4 antibody via the activation of N-hydroxysuccinimide and 1-ethyl-3(3-dimethylaminopropyl)carbodiimide. Resulting antibody-conjugated NPs (NPs-Ab) were then formulated into a $1 \%$ hydroxyethyl cellulose (HEC) vaginal gel.

Results: NPs-Ab showed a uniform particle size of $225.0 \pm 4.9 \mathrm{~nm}$, a zeta-potential of $-35.09 \pm 2.22 \mathrm{mV}$, an encapsulation efficiency of $63.0 \pm 5.7 \%$ and an antibody conjugation efficiency of $37.7 \pm 4.2 \%$. NPs showed a sustained release profile, with approximately $40 \%$ of siRNA released over 13 days. The NPs-Ab provided more than a 3 -fold increase in the intracellular accumulation of siRNA in the CD4+-cell line Sup-T1 compared to unconjugated NPs. 1\% HEC gel loaded with NPs-Ab demonstrated a non-Newtonian shear-thinning behavior and the viscosity of the NPs-Ab loaded gel was comparable to the over-the-counter lubricant gel products. Both the blank NPs $(1000 \mu \mathrm{g} / \mathrm{mL})$ and the $1 \%$ HEC placebo gel $(200 \mathrm{mg} / \mathrm{mL})$ had no significant impact on the viability of the vaginal epithelial cell line VK2/E6E7 after $24 \mathrm{~h}$ of exposure.

Conclusions: We have developed a novel intravaginal nano-based drug delivery system for the active delivery of siRNA to CD4+ immune cells. The NPs-Ab has desirable particle size for intravaginal delivery and sustained drug release. NPs-Ab can significantly increase the delivery of siRNA into CD4+ immune cells when compared to unconjugated NPs. NPs-Ab can be potentially be formulated into a gel dosage-form that is noncytotoxic with physical properties comparable to marketed vaginal gel products.

\section{Drug Releasing Targetable Thermosensitive Microgels}

Scott B. Campbell, and Todd Hoare

Department of Chemical Engineering McMaster University, Hamilton, ON

Purpose: Over the last few decades microgels have emerged as potentially useful vehicles for the release of therapeutics in vivo due to their injectability, their ability to extend the release kinetics of common therapies, and that they can be functionalized with ligands that allow them to be targeted to specific tissues. Thermoresponsive microgels offer a particularly attractive matrix for drug release in that the temperature-responsive swelling of such materials can be used to induce drug release on demand based on a heating stimulus. The incorporation of superparamagnetic iron oxide nanoparticles (SPIONs) into thermoresponsive microgel networks could thus not only allow for targeting capabilities but also some degree of external, on-demand control over the release kinetics.

Methods: Microgels were fabricated via a semibatch polymerization of $\mathrm{N}$-isopropyl methacrylamide (NIPMAM), acetoacetoxyethyl methacrylate (AAEM), and N,N'-methylene bisacrylamide (MBA), with 2-(dimethylamino) ethylacrylate (DMAEA) added slowly after the first hour of polymerization. The resulting purified microgels were then dispersed in deionized water at a concentration of $25 \mathrm{mg} / \mathrm{mL}$ and various amounts of iron salt solutions (with a 2:1 molar ratio between $\mathrm{FeCl}_{3}$ and $\mathrm{FeCl}_{2}$ ) were added to obtain microgels with $5-40 \quad$ wt $\%$ SPION contents. The nanocomposites are then loaded with fluorescein salts in a saturated solution and resulting fluorescein-loaded microgels will then be characterized via fluorescence spectroscopy for their release kinetics with and without exposure to magnetic heating via an external oscillating magnetic field.

Results: The microgels are thermosensitive in nature, experiencing a $56 \%$ decrease in volume from $25^{\circ} \mathrm{C}$ to $37^{\circ} \mathrm{C}$ and aggregating at $43^{\circ} \mathrm{C}$. MicrogelSPION nanocomposites with various SPION contents $(0 \%-40 \%)$ were stable over a long period of time (>2 weeks) in aqueous solutions (Figure 1). As more iron oxide is added, the average size of the microgel increases accordingly, from $145 \mathrm{~nm}$ without SPIONs to $250 \mathrm{~nm}$ at $25 \%$ SPION content while the thermosensitive characteristics of the 
materials are retained (the $15 \%$ SPION microgel experiences a $50 \%$ decrease in volume from $37^{\circ} \mathrm{C}$ to $43^{\circ} \mathrm{C}$, interestingly without aggregation occurring). Release kinetics of fluorescein will be presented as a function of SPION content and magnetic pulsing.

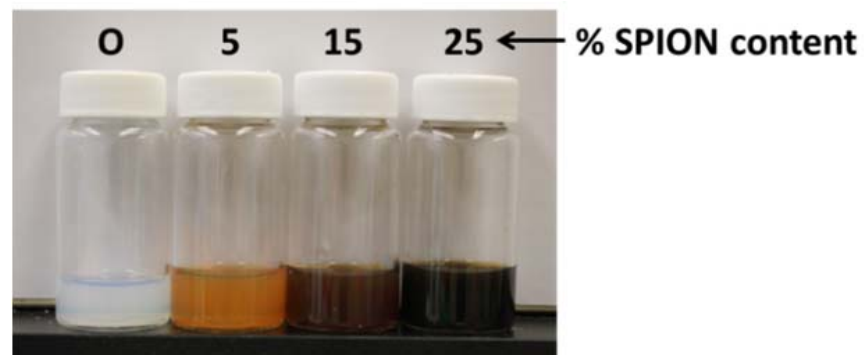

Figure 1: Nanocomposite microgels with varying SPION content.

Conclusions: Magnetic, thermosensitive, cationic nanocomposite microgels have potential for the triggered release of therapeutics. These microgelSPION nanocomposites offer potential as targetable drug delivery vehicles that could also be capable of externally-induced changes in release properties.

\section{The Effect of Polymer Charge and Architecture on Protein Release Kinetics of Injectable, Degradable Hydrogels}

Emilia Bakaic, Niels M.B. Smeets, Maryam Badv, and Todd Hoare

Department of Chemical Engineering, McMaster University, Hamilton, Ontario, L8S 4L7

Purpose: Control over the spatial and temporal delivery of drugs can improve efficiency of medical treatments and reduce side-effects for the patient. Minimally invasive, injectable drug delivery devices designed for sustained drug release offer favourable solutions for many therapeutic applications. Advances in controlled drug delivery (particularly within hydrogels) are limited by lack of fundamental understanding of the effect of gel morphology and charge on release kinetics, particularly with respect to prolonged protein release. We have designed injectable and degradable poly(oligoethylene glycol methacrylate) (POEGMA) hydrogels prepared with well-defined morphologies (linear, hyperbranched, and microgel polymers) and charge distributions (positive, negative, or amphoteric), aiming to identify structure-property correlations related to controlled release of protein-based therapeutics.
Methods: POEGMA hydrogels are prepared via coextrusion of synthetic hydrazide and aldehydefunctionalized poly(oligoethylene glycol methacrylate) (POEGMA) polymers (linear, hyperbranched or microgel architecture). Entrapped temperature responsive unfunctionalized microgels with a lower critical solution temperature of $32^{\circ} \mathrm{C}$ result in the creation of (more) hydrophobic nanodomains inside gels made from linear precursors. Gelation is achieved by the fast reaction between complementary reactive groups, which condense to form hydrolytically-degradable hydrazone bonds. Drug release kinetics were investigated by incorporating model drug BSA (bovine serum albumin) into the precursor solutions and tracking release kinetics via fluorescence measurements, with gels prepared at both $22^{\circ} \mathrm{C}$ (room temperature) and $37^{\circ} \mathrm{C}$ (in vivo conditions). Swelling (gravimetry), mechanical strength (rheometry), and cytotoxicity (MTT assay) were measured and related to release kinetics.

Results: Mechanical strength was governed by POEGMA polymer morphology and charge, with increasing storage modulus observed with microgel $<$ hyperbranched < charged hyperbranched < charged linear gels. Polymer architecture and charge greatly influenced the drug release kinetics of BSA, with release rate scaled with morphology as microgel $<$ linear $<$ hyperbranched $<$ charged linear. Interestingly, the amphoteric hydrogel displayed a significantly more prolonged release of BSA without a burst release, making this composition of interest for future optimization. MTT assays showed minimal cytotoxicity in vitro to $3 \mathrm{~T} 3$ mouse fibroblast cells when exposed to all composite formulations and all of their precursor materials. All systems were shown to be fully degradable. Extended drug release as well as drug phase partitioning experiments are currently being conducted.

Conclusion: The design of composite hydrogel systems based on polymer building blocks with well-defined charges and architecture offers an effective tool for controlled protein delivery. The injectable, degradable POEGMA composite hydrogels allows for tunable drug release kinetics governed by simple morphological and charge variations. 


\section{Injectable, In-situ Gelling, Cyclodextrin- dextran Hydrogels for the Partitioning-driven Release of Hydrophobic Drugs}

$\underline{\text { Rabia Mateen, Todd Hoare }}$

McMaster University, Hamilton, ON, Canada

Purpose: The effective controlled release of hydrophobic drugs from hydrogel-based delivery vehicles offer a persistent challenge in medicine. B-cyclodextrin offers a potentially attractive option to achieve this goal. In this study, an injectable dexamethasone-loaded (Dex- $\beta C D$ ) hydrogel was prepared and characterized. Gelation of these materials occurs in situ upon injection through the reaction of an aldehyde-functionalized dextran with a hydrazide-functionalized $\beta C D$ molecule. The dual role of $\beta C D$ as a cross-linker and host for the hydrophobic drug allows for the adjustment of hydrogel properties and release behavior.

Methods: $\beta C D$ was functionalized with hydrazide groups by the carbodiimide-mediated coupling of carboxymethylated $\beta \mathrm{CD} \quad(3.8 \quad \beta \mathrm{CD}-\mathrm{COOH})$ with adipic acid dihydrazide, generating a $\beta C D$ product possessing $\sim 3$ hydrazide groups. A hydrazidefunctionalized dextran polymer was synthesized in a similar fashion. Aldehyde-functionalized dextran was synthesized by sodium periodate-mediated oxidation. Hydrogels were formed by extruding equal amounts of hydrazide polymer, drug loaded $\beta C D-\mathrm{Hzd}$, and aldehyde polymer through the mixing device of a double barrel syringe into silicone rubber molds. The release study was carried out at $37^{\circ} \mathrm{C}$ over the course of 21 days.

Results: On average, $5.4 \%$ of the total drug was released from each Dex- $\beta C D$ gel $(11 \beta C D / 2 D e x-$ Hzd). The slow release of the drug can be attributed to the immobilization of the $\beta \mathrm{CD} / \mathrm{drug}$ complex within the hydrogel network. In comparison to the drug release observed from a dextran-based hydrogel prepared without $\beta C D$ (2Dex-Hzd), conventional burst release was observed. Interestingly, by doubling the concentration of the Dex-Hzd polymer in the gel precursor solution $(11 \beta \mathrm{CD} / 4 \mathrm{Dex}-\mathrm{Hzd})$, drug release was significantly enhanced (64\% total release compared to $5.4 \%$ total release aver 20 days). In this case, the hydrazide groups on the polymer will compete more effectively with those of the functionalized $\mathrm{CD}$, making $\mathrm{CD}$ less likely to crosslink and more likely to become physically entrapped in the hydrogel. Differences in release kinetics are thus attributed to the higher mobility of the complexed drug when $\beta C D$ is mostly physically entrapped in a hydrogel.

Conclusion: In hydrogel systems where $\beta \mathrm{CDs}$ cross-link the polymer network, a reduced drug release rate was observed. In hydrogel systems where $\beta C D$ was mostly physically entrapped within the matrix, an increased release rate was observed.

\section{Engineered Solid Lipid Nanoparticles of Paclitaxel for Effective Management of Lung Carcinoma}

\section{Vandana Soni}

Department of Pharmaceutical Sciences, Dr. H. S. Gour University, Sagar (MP) India

Purpose: Lung cancer is a dreadful disease which claims to be more life threatening as compared to total sum up of the other cancers. Thus there is an urgent need to develop an effective delivery approach for its management. paclitaxel (PTX) is one of the well known choice as antineoplasitc agent used for the treatment of different types of human cancers. Lactoferrin (Lf), a 'multifunctional protein' Lf receptors are expressed on the apical surface on bronchial epithelial cells. These over expressed LF receptors can be utilized for the transportation of Lf conjugated drug or nanocarrier devices. The present study was aimed to develop PTX loaded Lf coupled solid lipid nanoparticles (SLNs) for the treatment of lung cancer.

Methods: PTX loaded SLNs were prepared by solvent injection method and then coupled with Lf using carbodiimide chemistry. The formulations were characterized by transmission electron microscopy (TEM), particle size, polydispersity index (PDI) and zeta-potential, whereas Lf conjugation was confirmed by FT-IR and ${ }^{1} \mathrm{H}$ NMR and efficiency of prepared system was evaluated by in vitro, ex vivo and in vivo evaluations.

Results: The zeta potential of paclitaxel loaded SLNs were found to be approximately $6.8 \pm 0.2 \mathrm{mV}$ as compared to paclitaxel loaded Lf coupled SLNs $(3.7 \pm 0.3 \mathrm{mV})$.

The average particle size was studied by photon correlation spectroscopy and it was found to be $249.4 \pm 2.6 \mathrm{~nm}$ with coupled formulation. Polydispersity index of SLNs was found to less than one with both formulations.

The TEM photo micrographs depicted the nanometric size of both formulations. The drug entrapment efficiency of paclitaxel loaded SLNs was 
found to be $79.3 \pm 0.5 \%$ whereas in case of paclitaxel loaded Lf coupled SLNs, it was found to be $71.7 \pm 0.5 \%$.

The in vitro drug release studies depicted a biphasic drug release pattern which is an initial fast release followed by a slower sustained release.

The ex vivo cytotoxicity studies on BEAS-2B cell lines revealed superior anti-cancer activity of $\mathrm{Lf}$ coupled SLNs than plain SLNs and free PTX. In vivo biodistribution studies showed higher concentrations of PTX accumulated in lungs via Lf coupled SLNs than plain SLNs and free PTX.

Conclusion: These studies suggested that $\mathrm{Lf}$ coupled PTX loaded SLNs could be used as potential targeting carrier for delivering anti-cancer drug to the lungs with the minimal side effects. 


\section{Poster Session 2}

\section{CSPS and CC-CRS Posters}

\section{Thursday, June 12}




\section{Posters - Session 2}

\section{Thursday, June 12}

\section{Biomedical Sciences}

\section{Dietary Administration of Simvastatin Decreases Castration-Resistant Progression of the LNCaP Prostate Cancer Xenograft Model}

$\underline{\text { Jacob Gordon }}^{\mathrm{a}}$, Howard Tearle ${ }^{\mathrm{b}}$, Mazyar Ghaffari ${ }^{\mathrm{b}}$, Ankur Midha $^{\mathrm{a}}$, Michael Cox ${ }^{\mathrm{b}}$, Kishor Wasan ${ }^{\mathrm{a}}$

${ }^{a}$ Faculty of Pharmaceutical Sciences, University of British Columbia, Vancouver, BC; ${ }^{\mathrm{b}}$ The Vancouver Prostate Centre and Department of Urologic Sciences, University of British Columbia, Vancouver, BC

Funding Acknowledgments: CIHR and Prostate Cancer Canada

Purpose: Systemic androgen deprivation therapy is used to manage advanced metastatic prostate cancer, however the achieved remission is almost always temporary with patients recurring and progressing to castration-resistant prostate cancer (CRPC). Current therapeutic options for this lethal stage of the disease have had limited success. Recent evidence suggests that de novo steroidogenesis from the precursor cholesterol may play a role in the cancers progression to castration resistance. This study used the established LNCaP xenograft model for CRPC progression and development to test whether limiting cholesterol availability using simvastatin might suppress CRPC progression.

Method: $\mathrm{Nu} / \mathrm{Nu}$ mice were inoculated with $\mathrm{LNCaP}$ prostate cancer cells and allowed to develop tumours. Upon reaching a PSA of at least $50 \mathrm{ng} / \mathrm{ml}$, the animals were castrated and started on either a $0.1 \% \mathrm{w} / \mathrm{w}$ simvastatin chow or a standard diet. Tumour volume was assessed through caliper measurements once weekly. Circulating PSA levels were measured using a Cobas e-411 analyzer once weekly. Potential simvastatin liver and kidney toxicity was assessed by alanine transaminase (ALT) and creatine kinase assays (CK).

Results: Mice placed on the simvastatin chow showed reduced tumour volume growth, with the greatest differences being displayed from weeks 6-8 post castration (Figure 1). The simvastatin group also had a longer time to $400 \%$ of nadir PSA with almost half of the standard group reaching this level within 3 weeks post castration compared to $10 \%$ of the simvastatin group. The average CK and ALT activity of the simvastatin group $(282.32 \mathrm{U} / \mathrm{L}$ and $0.053 \mathrm{U} / \mathrm{ml}$, respectively) was indistinguishable from that of the standard group (289.51 U/L and $0.044 \mathrm{U} / \mathrm{ml}$, respectively) (Table 1 ).

Conclusion: The administration of simvastatin through the mice's diet resulted decreased the rate of post-castration tumour volume growth, increased time to PSA recurrence and had no measured toxicity. This highlights the potential applicability of a well established class of drug for the treatment of late-stage castration-resistant prostate cancer.

Figure 1

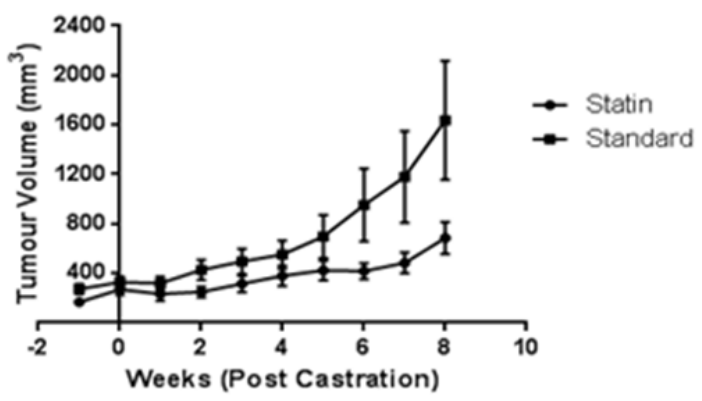

Table 1

\begin{tabular}{|c|c|c|c|c|}
\hline & \multicolumn{2}{|c|}{$\begin{array}{l}\text { CK Activity (+/- } \\
\text { SD) }\end{array}$} & $\begin{array}{l}\text { ALT } \\
(+/-\mathrm{SD})\end{array}$ & Activity \\
\hline Standard & $\begin{array}{l}289.51 \\
(79.41)\end{array}$ & $\mathrm{U} / \mathrm{L}$ & $\begin{array}{l}0.044 \\
(0.027)\end{array}$ & $\mathrm{U} / \mathrm{mL}$ \\
\hline Statin & $\begin{array}{l}282.32 \\
(36.79)\end{array}$ & $\mathrm{U} / \mathrm{L}$ & $\begin{array}{l}0.053 \\
(0.026)\end{array}$ & $\mathrm{U} / \mathrm{mL}$ \\
\hline
\end{tabular}




\section{MyoNovin Synthesis, Purification, Analysis and Bioactivity on Muscle Fibers from Zebrafish}

Siyan Wang, Drielly Cristina Braite, Sarah Montiel Gomes Eloi, Helia Zhang, Judy Anderson, Frank Burczynski

Faculty of Pharmacy, University of Manitoba, Winnipeg, Manitoba; Department of Biological Sciences, Faculty of Science, University of Manitoba; National Council for Technological and Scientific Development - CNPq

Purpose: MyoNovin is a novel skeletal muscle regenerator, first reported in 2009. MyoNovin is capable of activating muscle satellite (stem) cells to initiate myogenesis. Characterization of the molecule following synthesis requires a highperformance liquid chromatography (HPLC) assay to confirm purity and stability. We report on the synthesis, analysis, and bioactivity of MyoNovin using an in vitro muscle-fiber culture model.

Methods: MyoNovin was synthesized using a twostep reaction. The first step involved the addition of guaiacol, allybromide and potassium carbonate $\left(\mathrm{K}_{2} \mathrm{CO}_{3}\right)$ in acetone. The reaction was refluxed overnight at $78^{\circ} \mathrm{C}$. The product was concentrated using vacuum distillation to extract any volatile liquids. Thin layer chromatography (TLC) was used to test the purity of the intermediate. The second step involved reacting the obtained product with iodine $\left(\mathrm{I}_{2}\right)$ and silver nitrate $\left(\mathrm{AgNO}_{3}\right)$. The reaction was refluxed overnight at $90^{\circ} \mathrm{C}$. The final product was concentrated by vacuum distillation and analyzed by TLC. Purification of the final product was by column chromatography using silica gel in ethylacetate/hexane. HPLC and NMR were used to identify MyoNovin. MyoNovin was dissolved in DMSO. Muscle fibers were isolated from zebrafish and plated on coverslips. Fibers were incubated in $1 \mathrm{~mL}$ of basal growth media with $200 \mathrm{ng} \mathrm{BrdU} / \mathrm{mL}$ and treated with MyoNovin (1mM) or DMSO vehicle (control). After staining for BrdU, darkly stained nuclei in activated (replicating) satellite cells were counted per fiber.

Results: HPLC and NMR analyses identified MyoNovin; HPLC detected MyoNovin elution time and contaminants (if any). The MyoNovin peak appeared earlier in the solvent system using acetonitrile vs. methanol. The elution time for MyoNovin was then analyzed at different acetonitrile concentrations and injection-flow rates. Higher acetonitrile concentrations were associated with faster elution times. Activation of satellite cells (SC) was evaluated based on the mean number of BrdU+ SC per fiber. Fibers had a greater number of activated satellite cells when treated with MyoNovin compared to controls.

Conclusions: Acetonitrile at $60 \%$ in water as the mobile phase, proved to be a good option for eluting MyoNovin. SC on muscle fibers obtained from zebrafish demonstrated significant activation by MyoNovin treatment as shown by BrdU incorporation.

\section{Correlation between Arachidonic Acid Metabolites and Renin-angiotensin System peptides in Adjuvant Arthritis Rat}

Ali Aghazadeh-Habashi, Waheed Asghar, Fakhreddin Jamali

Faculty of Pharmacy and Pharmaceutical Sciences, University of Alberta, Edmonton, Canada

Purpose: Renin-angiotensin system (RAS) and arachidonic acid (AA) pathway as body's two main systems are present in all tissues and their balance is essential for homeostasis of renal and cardiovascular (CV) systems. RAS is comprised of two main peptides, angiotensin (Ang) II and Ang 1-7 that are produced by the angiotensin converting enzymes. AA metabolism yields 20-hydroxyeicosatetraenoic acid (20-HETE), epoxyeicosatrienoic acids (EETs) and dihydroxyeicosatrienoic acids (DHTs). Pathological conditions such as inflammation alter the balance between these two systems. The knowledge about status of RAS and AA pathways components and the correlation between them is critical for identification of the individuals at risk of renal or $\mathrm{CV}$ complications. In this study, we aimed to identify some plasma components of these systems as biomarkers.

Method: Sprague Dawley rats were divided into 2 groups ( $\mathrm{n}=3$ /group) of healthy control and inflamed. Inflamed groups were injected with Mycobacterium butyricum in squalene in their tail base. After 16 days, when signs of arthritis emerged animals were euthanized, plasma, hearts and kidney were harvested and stored at $-80^{\circ}$ until analyzed for Ang peptides and AA metabolites using ELISA and HPLC-FL, respectively. Plasma AA metabolites levels were correlated against tissue Ang peptides concentrations.

Results: There were strong correlations between plasma levels of eicosanoids and Ang peptides in 
different tissues.

Conclusion: The findings of present work suggest plasma Ang II, Ang 1-7, 20-HETE, 14-15 EET, 1415 DHT, total DHTs, 20-HETE/EETs and DHTs/EETs could be used as surrogate biomarkers for identification of individual at risk of developing renal or $\mathrm{CV}$ disease.

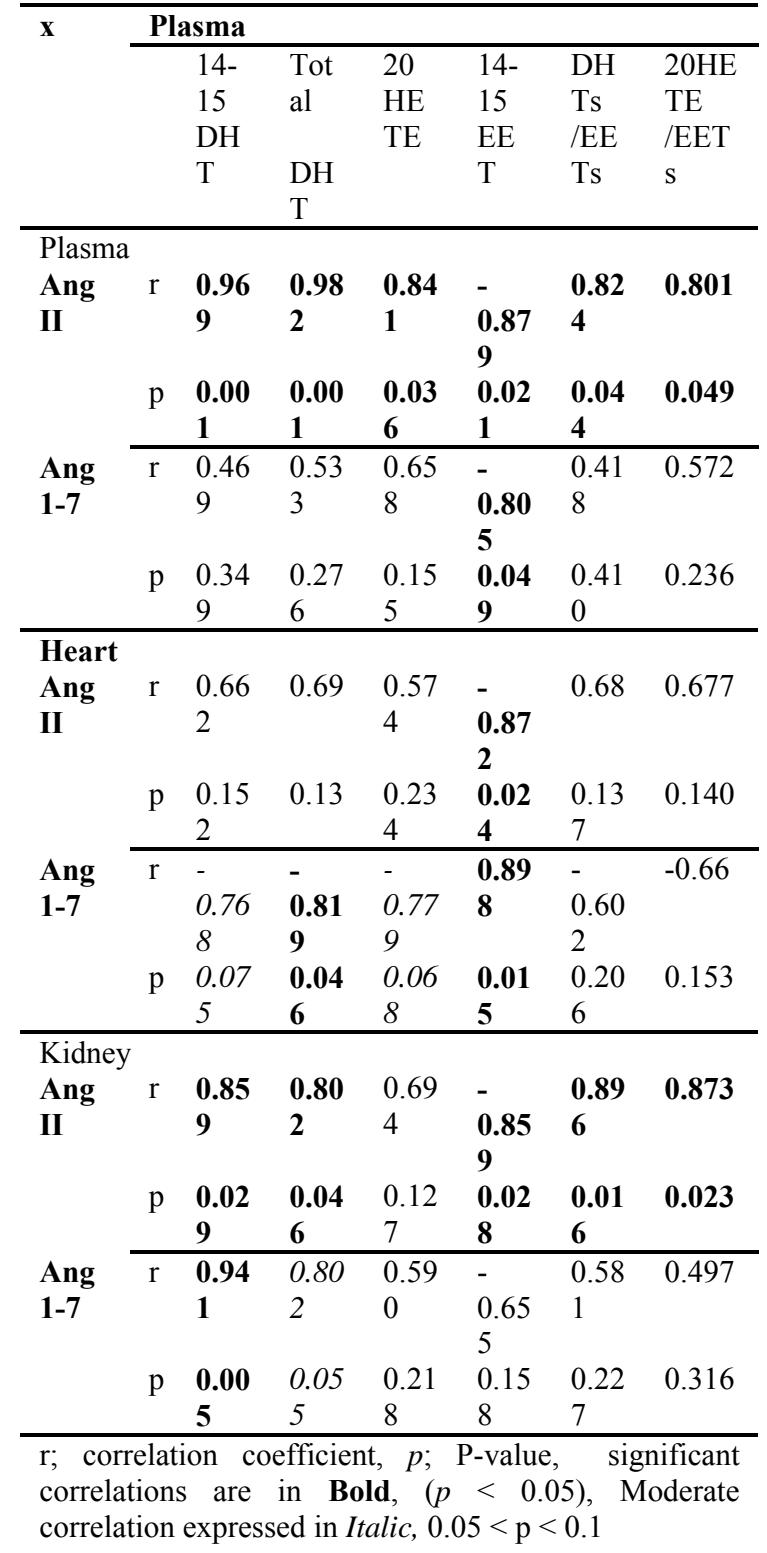

\section{Significance of Bone Morphogenetic Proteins for Understanding of Traditional Chinese Herbs in Treatment of Liver Disease}

Xiaotong Wang ${ }^{1}$, Ping $\mathrm{Li}^{1}$, Frank J. Burczynski ${ }^{2}$, Neal Davies ${ }^{2}$, Yuewen Gong ${ }^{2}$

1. China-Japan Friendship Hospital, Beijing, P.R. of China; 2. Faculty of Pharmacy, University of Manitoba, Winnipeg, Canada

Purpose: Bone morphogenetic proteins (BMPs) are a group of growth factors belonging to the superfamily of transforming growth factor beta. Our previous findings showing significantly increased BMP4 levels in bile duct ligated livers and increased expression of BMP4 mRNA in activated hepatic stellate cells, which are responsible for the development of fibrosis in the liver. Several traditional Chinese herbs have been identified to reverse liver fibrosis. However, the mechanism(s) of these herbs in the treatment of liver fibrosis remains to be investigated.

Methods: By employing hepatic stellate cell line (LX-2 cells) and hepatoma cell line (Huh-7 cells), we investigated the regulation of BMP4 expression with several components of these herbs. BMP4 expression was evaluated by RT-PCR and Western blot analyses and WST-1 proliferation reagent determined cell proliferation.

Results: We employed saikosaponin-d (SSd) and paeoniflorin (PF) extracted from the bupleurum and Paeonia lactiflora to treat LX-2 and Huh-7 cells respectively. SSd significantly inhibited LX-2 cell proliferation and PF inhibited Huh-7 cell proliferation, respectively. Although BMP4 did not affect both LX-2 and Huh-7 cell proliferation, both SSd and PF reduced BMP4 expression in both cells. Moreover, BMP4 promoted hepatic stellate cell trans-differentiation as shown in elevated level of alpha smooth muscle actin ( -SMA). SSd inhibited stellate cell trans-differentiation (decreased -SMA level). Furthermore, SSd also increased the level of BAX in LX-2 cells.

Conclusion: BMP4 could be the growth factor involved in the regulation of hepatic stellate cells and hepatoma cells that is perturbed by this treatment. 


\section{Probing the Efficiency and Linkage Specific Cleavage Activity of Sialidase in Micromonospora viridifaciens}

Saeideh Shamsi, Kazem Abadi ${ }^{1}$, Andrew J. Bennet ${ }^{2}$ ${ }^{1}$ Department of Molecular Biology \& Biochemistry, Simon Fraser University, Burnaby, BC, Canada; ${ }^{2}$ Department of Chemistry, Simon Fraser University, Burnaby, BC, Canada

Purpose: Sialic acids are often found at the terminal positions on the glycan chains that adorn all vertebrate cells and glycoproteins. This prominent position confers an essential role to sialic acid residues in biology, evolution and disease propagation. Glycoside hydrolase family 33 (GH33) contains exo-sialidases (E.C. 3.2.1.18, neuraminidases), from both eukaryotes and prokaryotes, that catalyze the hydrolysis of sialic acid from glycoconjugates. On cell surfaces, sialic acids are often attached in a $\alpha 2-6$ or $\alpha 2-3$ linkage to the penultimate sugar, which is frequently a galactoside, and these different linkages are often selectively recognized by neuraminidases. From a glycobiology point of view there is great interest in isolation or evolution of a sialidase which would exclusively hydrolyze $\alpha 2-6$ linkages as there is an abundant presence of $\alpha 2-6$ linked sialic acids on human cell surfaces.

Methods: To this end we took advantage of a subgroup of sialidases, called trans-sialidases that transfer sialic acid directly from one glycosidic linkage onto another sugar residue instead of a water molecule. An overlay of the structure of $M v \mathrm{NA}$ with Trypanosoma cruzi trans-sialidase (TcTS), the agent of Chagas' disease in humans and animals, reveals the presence of several loops in the TcTS believed to aid the enzyme in linkage specific transfer.We have created $M v$ NA mutants containing the relevant loops and will be generating libraries of saturation mutations of the amino acids in these loops. By monitoring the hydrolytic activity of mutant enzymes in the presence of 8FMU $\alpha$-Neu5Ac$(2 \rightarrow 6)-\beta-d-G a l p$ and 8FMU $\alpha-N e u 5 A c-(2 \rightarrow 3)-\beta-d-$ Galp we will identify clones with linkage specific cleavage activity.

Results: We have identified several clones with modified 2,3 and 2,6 cleavage activity and we will be testing these clones to determine the specific enzyme activity of each clone. This will in turn aid in choosing a clone for further active site mutagenesis.
Conclusion: Despite their documented role of sialic acid in numerous diseases, our knowledge of the functions and metabolism of certain sialoglycans, linkage specific attachment or cleavage of sialic acids to cellular glycans are scant and require further in depth research to delineate the role of various sialic acid structures. Evolution of an enzyme with 2,6 linkage specific hydrolysis activity is essential in elucidation of the role of cleavage specific sialidation of glycoproteins and their role in disease propagation.

\section{Clinical Sciences \& Pharmacy Practice}

\section{In Search of the Optimal Strategy for Sedative Discontinuation: A Literature Review}

André Pollmann, Andrea Murphy, and David Gardner

College of Pharmacy, Dalhousie University, Halifax, Nova Scotia, Canada

Purpose: Long-term sedative use, especially in older adults, is associated with significant morbidity. Multiple approaches to stopping long-term sedative treatment have been proposed. We conducted a literature review to map, synthesize, and ultimately explore best-practices for optimal methods of chronic benzodiazepine and similar sedative therapy discontinuation.

Methods: A systematic database search of PubMed, Cochrane Central Register of Controlled Trials (CENTRAL), EMBASE, PsycINFO, and TRIP was conducted. Search strategies were developed and reviewed by a library scientist. Search terms were derived from medical subject headings, key words from previous systematic reviews and metaanalyses, and tacit knowledge on the subject. Grey literature searches were systematic using multiple sources (e.g., Google Advanced, Grey Matters from the Canadian Agency for Drugs and Technologies in Health). Experimental, quasi-experimental, and observational studies, except for case-reports, and policies and reports were eligible for inclusion. Searches were limited to English language.

Results: Following removal of duplicate articles, 
1638 article titles and abstracts were reviewed; four randomized controlled trials and one meta-analysis were selected as the best available evidence to guide benzodiazepine and similar sedative therapy discontinuation. Seventy-five websites were found and within these, 64 of 3897 hits related to benzodiazepine and similar sedative therapy discontinuation were potentially relevant. Several methods, including use of adjunctive medications, tapering schedules, cognitive behavioural therapy, other psychological interventions, and combinations of interventions have been studied. Structured gradual dose reduction (GDR) appears to be promising in facilitating discontinuation with a relative risk for complete discontinuation at 12 months of 4.97 (95\% CI 2.2-11.1) and a number needed to treat of three. Different rates of GDR have not been compared in any of the studies identified and taper schedules employed vary among studies, are commonly flexible, and patient specific.

Conclusions: A slow, flexible taper with frequent follow-up appears to be the leading method supporting successful benzodiazepine and similar sedative therapy discontinuation. Adjunctive therapies may provide a small additional benefit. The best method of sedative discontinuation could not be determined with certainty due to the lack of comparative studies and heterogeneity across studies. This research provides foundations in moving forward with a formal scoping review of the literature.

Acknowledgement: André Pollmann received the National Summer Student Research Program Awards to Present Research Findings sponsored by GlaxoSmithKline Inc.

\section{Pharmacy Students-As-Immunizers: A Scoping and Jurisdictional Review}

$\underline{\text { Sarah Johnson, Nancy Waite, and Eric Schneider }}$ School of Pharmacy, University of Waterloo, Waterloo, Ontario, Canada

Purpose: Despite universal vaccine availability, vaccination rates have stagnated at suboptimal levels. To address this issue, several Canadian provinces and all US states have expanded the scope of pharmacists to provide vaccinations. Pharmacy students represent another potential vaccine administrator and inclusion of this role during their undergraduate training has the potential to both assist pharmacist vaccination efforts and improve students' readiness to provide these services upon graduation. The goal of this initiative is to review the literature on pharmacy students-as-immunizers and the regulatory authority. The results of this report may help inform Canadian policy recommendations.

Methods: Data was collected to determine which Canadian provinces and US states have legislation allowing pharmacy students to immunize, which vaccines they are permitted to administer, what training is required and what level of supervision is mandated. A scoping review was also conducted to examine the student, patient and community benefits of pharmacy students-as-immunizers.

Results: Four Canadian provinces and 39 US states have legislation permitting students to administer vaccines. In all cases, students must be under the supervision of a pharmacist, usually an authorized immunizer. In many jurisdictions, pharmacy students must have immunization training and be certified in first aid. The literature provides a variety of strategies for utilizing students-as-immunizers including embedding this practice in rotations, student-run clinics, and involvement in health fairs and community based clinics.

Conclusions: Pharmacy students-as-immunizers is a mechanism which will increase the availability and accessibility of vaccines; potentially benefiting the student, the patient and the profession. Specific policy recommendations will be summarized.

Acknowledgement: Sarah Johnson received the National Summer Student Research Program Awards to Present Research Findings sponsored by GlaxoSmithKline Inc.

47. Exposure to Rufinamide and Risks of CNS Adverse Events in Drug-Resistant Epilepsy: A Systematic Review and Meta-Analysis of Randomized Placebo-Controlled Studies

$\underline{\text { Abdulaziz M. S. Alsaad }}^{1,2}$ and Gideon Koren ${ }^{1}$

1. Motherisk Program, Division of Clinical Pharmacology and Toxicology, Leslie Dan Faculty of Pharmacy, Hospital for Sick Children, University of Toronto, Canada. 2. Department of Pharmacology \& Toxicology, College of Pharmacy, King Saud University, Riyadh, KSA.

Purpose: Epilepsy is a complex disease necessitating continuous development of new therapeutic strategies to encounter drug-resistant cases. Among the new adjuvant antiepileptic drugs, rufinamide is considered a novel compound that is 
structurally distinct from other antiepileptic drugs. It is used to treat partial-onset seizures and seizures associated with Lennox-Gastaut syndrome in adult and children. Although the effectiveness of rufinamide has been studied, to date, there has been no attempt to systematically evaluate the risks of rufinamide's adverse events.

Method: We performed a quantitative risk analysis of the central nervous system (CNS) adverse events of rufinamide from all randomized, double blind, add-on, and placebo-controlled trials. Of the 886 publications reviewed, 99 papers were retrieved and five articles met the inclusion criteria. Around 1252 patients were included in this meta-analysis.

Results: Our study showed that exposure to rufinamide is associated with a significant increase in risk of somnolence (RR $1.87 ; 95 \%$ CI 1.33 to 2.62; $\mathrm{P}=0.0003)$, dizziness $(2.66 ; 2.00$ to 3.55 ; $\mathrm{P}=0.00001)$, fatigue/lethargy $(2.14 ; 1.57$ to 2.91 ; $\mathrm{P}=0.01)$, and headache $(1.28 ; 1.02$ to $1.59, \mathrm{P}=0.03)$. In addition, exposure to rufinamide was associated with higher treatment discontinuation rates as compared to placebo $(2.65 ; 1.74$ to 4.03 ; $\mathrm{P}=0.00001$ ).

Conclusions: The risk of CNS adverse events appears to be increased in patients exposed to rufinamide as well as the treatment discontinuation rates. However, although statistical association was significant, additional studies are required to confirm clinical significance of these findings, as most reports involved mild to moderate adverse events. Ultimately, to help in clinical decision-making process, this study would be an important consideration when choosing the appropriate adjuvant therapy for the next patient with epilepsy, with Lennox-Gastaut syndrome or not.

\section{Cardiovascular Safety of NSAIDs: A Systematic Review of Meloxicam}

Waheed Asghar, Fakhreddin Jamali

Faculty of Pharmacy \& Pharmaceutical Sciences, University of Alberta, Edmonton AB

Purpose: Non-steroidal anti-inflammatory drugs (NSAIDs) are used to treat inflammatory disorders. Conflicting evidence from clinical trials have suggested that long term use of NSAIDs can cause cardiovascular (CV) complications. Although human data is limited, animal studies have shown that the drug distribution into target organs may be, at least in part, associated with differences in $\mathrm{CV}$ risk of
NSAIDs. For example meloxicam, owing to its limited distribution into the kidney, is reported to cause less renal function abnormalities in the rat. The purpose of this systematic review was to assess the overall $\mathrm{CV}$ risks while stratified into different categories of coronary, vascular and renal outcomes.

Methods: We conducted an online search in selected healthcare database (till June 2013) looking for comparative observational studies or randomized clinical trial, having meloxicam in their comparisons and reporting coronary outcome (upon $>90$ days exposure) or vascular/renal outcomes (upon any exposure). Our search recruited 2106 studies out of which 17 were eligible (10 case control, 3 cohort and 4 randomized control trials) reporting coronary $(n=5)$, vascular $(n=6)$ and renal $(n=6)$ outcomes. The odd ratio (OR, 95\% CI) and relative risk (RR, 95\% CI) data was abstracted and pooled using fixed effect inverse variance model.

Results: Meloxicam caused moderate but significant overall CV risk $1.15[1.05,1.25]$ mainly due to an elevated vascular $1.37[1.21,1.55]$ risk; however, the coronary $1.00[0.87,1.14$ and renal $0.83[0.63,1.11]$ risks were not increased. Rofecoxib causes the highest overall CV risk $1.38[1.29,1.46]$ that includes higher coronary $1.46[1.10,1.93]$, vascular $1.31[1.21,1.41]$ and renal $1.51[1.35,1.68]$ risks. Other NSAIDs increased the overall risk in the following order indomethacin $>$ diclofenac $>$ naproxen $>$ celecoxib $>$ ibuprofen. Etodolac causes no increase in either coronary or renal risk but data is lacking on its vascular outcomes. The available studies often overestimate the NSAIDs CV risk, due to a lack of adjustment for the undermining arthritis that by itself is associated with CV events.

Conclusion: Evidence suggests that meloxicam and etodolac causes lesser overall $\mathrm{CV}$ risks than other NSAIDs. The available information on the relative $\mathrm{CV}$ risks of the NSAIDs may be overestimated because 1) they may be generated in comparison to other NSAIDs used and/or 2) they may completely ignore the acknowledged $\mathrm{CV}$ risks of inflammatory diseases such as arthritis. 


\section{Pharmacokinetics \& Pharmacodynamics}

\section{Cytochrome P450 Metabolites of Arachidonic Acid in Plasma \& Heart as Bio-Marker of NSAIDS Induced Cardiovascular Risk in Adjuvant Arthritis Rat}

\begin{abstract}
Waheed Asghar, Ali Aghazadeh-Habashi, Fakhreddin Jamali

Faculty of Pharmacy and Pharmaceutical Sciences, University of Alberta, Edmonton, Canada
\end{abstract}

Purpose: Elevation of cardiotoxic 20hydroxyeicosatetraenoic acids (20-HETE, cardiotoxic) and reduced epoxyeicosatrienoic acids (EETs i.e. cardioprotective) are reported in inflammation and cardiovascular diseases. Epidemiological studies have shown that among non-steroidal anti-inflammatory drugs (NSAIDs) rofecoxib and flurbiprofen are more cardiotoxic than meloxicam and celecoxib. We hypothesize that eicosanoids can be used as biomarkers to differentiate between NSAIDs in their cardiovascular risk. Methods: Sprague-Dawley rats were divided into control, inflamed and inflamedtreated groups and the latter was subdivided to rofecoxib $10 \mathrm{mg} / \mathrm{kg}$, meloxicam $0.5 \mathrm{mg} / \mathrm{kg}$, celecoxib $15 \mathrm{mg} / \mathrm{kg}$ and flurbiprofen $5 \mathrm{mg} / \mathrm{kg}$. The inflamed group received Mycobacterium butyricum/squalene. After 7 days of treatment, blood and hearts were harvested and analyzed by HPLCFL method. Results: Inflammation resulted in altered plasma HETE/EET ratio toward cardiotoxicity. NSAIDs further elevated the ratio in the following order: Rofecoxib=flurbiprofen $>$ meloxicam= celecoxib.

\begin{tabular}{|l|l|l|}
\hline $\begin{array}{l}\text { Group }(\mathrm{n}=4 \\
\text { rats })\end{array}$ & Plasma (ng/ml) & $\begin{array}{l}\text { Heart (ng/mg of } \\
\text { tissue) }\end{array}$ \\
\cline { 2 - 3 } & $\begin{array}{l}\text { HETE/ EET } \\
\text { ratio }\end{array}$ & HETE/ EET ratio \\
\hline Control & $0.38(0.08)$ & $0.09(0.06)$ \\
\hline Inflamed & $0.84(0.28)^{\mathrm{a}}$ & $0.10(0.02)$ \\
\hline Rofecoxib & $4.04(0.69)^{\mathrm{a}, \mathrm{b}}$ & $0.32(0.11)^{\mathrm{a} . \mathrm{b}}$ \\
\hline Flurbiprofen & $3.69(1.53)^{\mathrm{a}, \mathrm{b}}$ & $0.29(0.07)^{\mathrm{a} . \mathrm{b}}$ \\
\hline Meloxicam & $1.88(0.56)^{\mathrm{a}}$ & $0.09(0.08)$ \\
\hline Celecoxib & $1.85(0.73)^{\mathrm{a}}$ & $0.09(0.06)$ \\
\hline $\begin{array}{l}\text { a, significant vs control, b, significant vs inflamed } \\
(\mathrm{p}<0.05)\end{array}$ \\
\hline
\end{tabular}

a, significant vs control, $b$, significant vs inflamed $(\mathrm{p}<0.05)$
50. Stereospecific Characterization of the PreClinical Pharmacokinetics, Pharmacological Activities and Content Analysis in Natural Health Products of 8-Prenylnaringein, A Potent Phytoestrogen Found in Hops (Humulus Lupulus)

Stephanie E. Martinez, Casey L. Sayre, Grace E.C. Frankel, Neal M. Davies

Faculty of Pharmacy, University of Manitoba, Winnipeg, Manitoba, Canada

Purpose: 8-Prenylnaringenin is a chiral prenylated flavonoid phytoestrogen found in the female flowers of hops (Humulus lupulus) as well as beer and natural health products including menopause support, breast enhancement and sleep aids. The purpose of these studies are to develop and validate an enantioselective analytical assay to quantify 8prenylnaringenin in biological fluids, to evaluate the enantiospecific pharmacokinetic disposition in a rodent model, to examine pharmacological activities of 8-prenylnaringenin and to evaluate its content in commercially available natural health products.

Methods: A stereospecific LC-ESI-MS method was developed and utilized to quantify 8prenylnaringenin in rat serum and urine. A Chiralpack $^{\circledR}$ AD-RH column with an isocratic mobile phase consisting of 2-propanol and $10 \mathrm{mM}$ ammonium formate $(\mathrm{pH} 8.5)(40: 60, \mathrm{v} / \mathrm{v})$ and a flow rate of $0.7 \mathrm{~mL} / \mathrm{min}$ was employed. Detection was achieved using negative selective ion monitoring of 8-prenylnaringein at $\mathrm{m} / \mathrm{z} 339.15$. Male CD (SpragueDawley) rats were dosed with either $10 \mathrm{mg} / \mathrm{kg}$ or $100 \mathrm{mg} / \mathrm{kg}$ intravenously or orally, respectively, with racemic 8-prenylnaringein. Serum and urine samples were collected over a $120 \mathrm{~h}$ period. Antioxidant capacity, COX-1 and -2 inhibition, and CYP2D6 inhibition by 8-prenylnaringein were evaluated using commercial assay kits. $\alpha$ Glucosidase and $\alpha$-amylase inhibition were evaluated using colorimetric assays. Content analysis of 39 natural health products advertised to contain 8-prenylnaringenin or $H$. lupulus was carried out using methanolic extractions.

Results: The LC-ESI-MS method was successfully validated and applied to stereospecific pharmacokinetic studies of 8-prenylnaringenin in the rat. After oral and intravenous administration, both enantiomers of 8-prenylnaringenin were detected as the parent compounds and the glucuronidated metabolites. Oral bioavailability was found to be $\sim 13 \%$ and renal excretion was found to be $\sim 1.5 \%$ of 
the dose. 8-Prenylnarignein possessed concentrationdependent antioxidant and COX activity. Poor $\alpha$ amylase and moderate dose-dependent $\alpha$-glucosidase inhibitory activities were observed. 8Prenylnaringein does not appear to significantly inhibit CYP2D6. 8-Prenylnaringein was detected in all but one natural health product but concentrations were inconsistent with labels.

Conclusions: A novel, sensitive LC-ESI-MS method was developed and validated for enantiospecific detection of 8-prenylnaringein in biological fluids. Pharmacokinetic studies of 8prenylnaringenin indicate differences in enantiomer disposition. 8-Prenylnaringein appears to possess concentration dependent antioxidant, COX inhibitory and $\alpha$-glucosidase inhibitory activities and poor $\alpha$-amylase and CYP2D6 inhibitory activities. Variability among 8-prenylnaringein natural health product content suggests lack of manufacturing quality control.

\section{Pharmaceutical \& Analytical Chemistry}

\section{Hepatoprotective Properties of Commiphora Opobalsamum (Balessan), A Traditional Medicinal Plant of Saudi Arabia}

Tawfeq Alhowiriny ${ }^{1}$, Mohammed Al-Sohaibani ${ }^{2}$, Mansour Al-said ${ }^{1}$, Mohammed Al-Yahya ${ }^{1}$, Kamal El-Tahir ${ }^{1}$, and Sayed Rafatullah ${ }^{1}$

1. Medicinal, Aromatic and Poisonous Plants Research Center, College of Pharmacy, King Saud University, Riyadh, Saudi Arabia; 2. Department of Pathology, College of Medicine, King Khalid University Hospital, King Saud University, Riyadh, Saudi Arabia.

Purpose: Balessan is the Arabic local name of Commiphora opobalsamum that has been used in diseases of liver, stomach, and urinary tract. The hepatoprotective activity of an ethanolic extract of C. opobalsamum was investigated in rats by inducing hepatotoxicity with carbon tetrachloride:liquid paraffin (1:1).

Methods: The aerial parts of the plant were collected in 2011. Powders from shade-dried plant sample (300 gm) were macerated in 96\% ethanol (4 liters) for $36 \mathrm{hrs}$ to yield the plant extract. Wistar albino rats of either sex were divided into four groups containing six animals in each group. Group I was kept as a control group. Groups II, III, and IV received $0.25 \mathrm{ml}$ of $\mathrm{CCl}_{4}$ in liquid paraffin (1:1) per $100 \mathrm{~g}$ body weight intraperitoneally. Group II received only $\mathrm{CCl}_{4}$ treatment. Groups III \& IV were treated in addition with 250 and $500 \mathrm{mg} / \mathrm{kg}$ of ethanolic extract of Balessan, respectively. The drug treatment was started 5 days prior to $\mathrm{CCl}_{4}$ administration and continued until the end of experiment.

Results: The preliminary qualitative phytochemical screening of the aerial parts revealed the presence of flavonoids, tannins, sterols and triterpenes. Rats subjected to the $\mathrm{CCl}_{4}$ regimen alone developed significant hepatocellular damage as evidenced by a significant elevation in serum activities of glutamate oxaloacetate transaminase, glutamate pyruvate transaminase, alkaline phosphatase and billirubin compared with normal values, which have been used as reliable markers of hepatotoxicity. Oral administration of an ethanol extract of Balssan exhibited a significant reduction in $\mathrm{CCl}_{4}$ induced increased levels of SGOT, SGPT, ALP and bilirubin concentrations.

Discussion: The plant extract had a significant protective effect by lowering serum transaminase levels (Serum glutamate oxaloacetate transaminase and serum glutamate pyruvate transaminase), alkaline phosphatase and billirubin. Regarding the effect of $\mathrm{CCl}_{4}$ on liver cells and the protective effect of C. opobalsamum, no confluent necrosis was observed in either Balessan extract-treated groups, which support our biochemical findings. The chemical constituents responsible for its hepatoprotective activity against chemical injury are not known. However, the plant contains a number of phytochemical constituents, including flavonoids, saponins, sterols and triterpenes. These data suggest that the plant $C$. opobalsamum may act as an antioxidant agent and may have a hepatoprotective effect. 


\section{Chemical and Biological Study of Flueggea virosa Native to Saudi Arabia}

Adnan J. Alharbi, ${ }^{1}$ Muhammad Yousaf, ${ }^{1}$ Mohammad S. Ahmad, ${ }^{1}$ Volodymyr Samoylenko, ${ }^{2}$ Xing-Cong Li, ${ }^{2}$ Ilias Muhammad ${ }^{2}$ and Kamal E. H. ELTahir $^{3}$

${ }^{1}$ Department of Pharmacognosy, College of Pharmacy, King Saud University, Riyadh, Saudi Arabia; ${ }^{2}$ National Center for Natural Products Research, Research Institute of Pharmaceutical Sciences, School of Pharmacy, University of Mississippi, University, MS, USA; ${ }^{3}$ Department of Pharmacology, College of Pharmacy, King Saud University, Riyadh, Saudi Arabia

Purpose: The objective of the study was to isolate and identify the major markers constituents of Flueggea virosa and to evaluate their antimicrobial, antiparacitic and cytotoxic potential.

Methods: The n-hexane and ethyl acetate fractions of the ethanol extract of the aerial parts of Flueggea virosa was subjected to repeated silica gel columns chromatography. The in vitro antileismanial assay was done on culture of Leishmania donovani promastigotes by Alamar Blue assay, while the in vitro antiplasmodial activity was measured by a colorimetric assay. The antimicrobial activity was performed using a modified version of the CLSI methods. In addition, cytotoxic activity was determined against two human cancer cell lines, KB and BT-549.

Results: A total of fourteen compounds were isolated and identified, which include the major compounds 1-7 [bergenin (1), menisdaurin (2), miroside (3), ent-phyllanthidine (4), securinine (5), securinol (6), viroallosecurinine (7)] along with rutin, two catechins gallocatechin (as a mixture), epi-gallocatechin, 2,2'5,5'-tetrahydroxybiphenyl, ethylmanopyranoside and ethylglucopyranoside (as acetates) and squalene. The alkaloids, securinine and viroallosecurinine showed marginal antiplasmodial activity and inactive against Leishmania donovani promastigotes, human cancer and non-cancerous VERO \& LLC-PK1 cells.

Conclusion: The isolation and identification of the major marker constituents 1-7 will allow us to develop an HPLC analytical method for quality control of $F$. virosa extracts.

\section{HPLC Analysis of Ciprofloxacin in Fish Antibiotics}

C. Farah $^{1}$ and P.M. D'Aoust ${ }^{2}$, A. Krantis ${ }^{3}$, J. T. Arnason $^{1}$, B. C. Foster ${ }^{3}$

${ }^{1}$ Faculty of Science, ${ }^{2}$ Faculty of Engineering and

${ }^{3}$ Faculty of Medicine, University of Ottawa, Ontario, Canada

Purpose: It has come to our attention that the Internet promotes self-medication with fish antibiotics. Information about obtaining and using fish antibiotics are easily found in online discussion boards on the Internet. This was evidenced by a letter to the editor in The New England Journal of Medicine recounting a soldier who self-medicated with fish antibiotics, and multiple online accounts promoting these products for human use. As these products are not regulated for use in humans, there is no guarantee as to quality or potency. The present study aims at analyzing and quantifying the active ingredient in ciprofloxacin (CFX) tablets purchased from online retailers.

Methods: Five CFX products were purchased (Thailand - 3, 1 each from Russia and Ukraine) from online retailers where they were sold and advertised as fish grade antibiotics. Five pills from each sample are tested for their ciprofloxacin content. CFX content relative to authentic standard, and the presence of related substances and contaminants were determined by HPLC at different wavelengths, $\mathrm{n}=5$. Tablet weights were also measured.

Results: All products contained CFX with no evidence of related substances or contaminants. Thirteen of 25 pills contained $90-110 \%$ active ingredient; only 2 of the products had a mean content within this range. Of the 12 resulting tablets below this range, 10 contained $80-90 \%$ active ingredient and 2 contained $76-80 \%$ active ingredient. No tablet contained more than $100 \%$ of the label weight. The tablet weights were generally uniform, CV 2.4\%. The $500 \mathrm{mg}$ tablets were most variable with a mean weight of $791 \mathrm{mg}$ with weights ranging from 756 to $842 \mathrm{mg}$.

Conclusion: The products tested were not adulterated and contained CFX. The majority of tablets did not meet the USP content standard. The sub-par nature of the samples is worrisome knowing that individuals self-medicating with these products would have difficulty maintaining proper therapeutic levels throughout treatment. 


\section{Carboxymethyl and Hydrazide Functionalized $\beta$-Cyclodextrin Derivatives: A Systematic Investigation of Complexation Behaviors with the Model Hydrophobic Drug Dexamethasone}

$\underline{\text { Rabia Mateen, Todd Hoare }}$

McMaster University, Hamilton, ON, Canada

Purpose: In this study, the complexation behavior of carboxymethylated $\beta \mathrm{CD}(\mathrm{CM} / \beta \mathrm{CD})$, hydrazidefunctionalized $\beta C D \quad(\mathrm{Hzd} / \beta \mathrm{CD})$ and a $\beta C D$ functionalized dextran polymer (Dex- $\beta C D$ ) were investigated, using the moderately hydrophobic drug dexamethasone $(\log P=1.77)$ as a model guest.

Methods: The carboxymethylation of $\beta C D$ was carried out for different time intervals and reaction cycles, depending on the desired degree of functionalization of the derivative. Hydrazide functionalized derivatives were synthesized from a $\mathrm{CM} / \beta C D$ derivative precursor with a similar degree of substitution. $\beta C D$ s were conjugated to aldehyde functionalized dextran via hydrazone linkages. Phase solubility studies were performed according to the methods of Higuchi and Connors. The stoichiometries of the $\beta C D$ derivative inclusion complexes were determined by the continuous variation method using data generated from the hostguest interaction of $\beta C D$ derivatives with 8-Anilino1-naphthalenesulfonic acid. The calorimetric titration of $\beta C D$ derivatives into dexamethasone was used to measure binding enthalpies of the host-guest interaction and were fitted to a one-site binding model. The size of $\beta C D$ derivative-drug complexes or associated aggregates was analyzed using the NanoSight single nanoparticle tracker.

Results: Functionalization of $\beta C D$ groups does not significantly change the stoichoimetry of the binding interactions. The least functionalized $\mathrm{CM}$ derivative $(1.7 \mathrm{CM} / \beta \mathrm{CD})$ has the highest $K_{a}$ value $(2450 \pm 180$ $\mathrm{M}^{-1}$ ) and complexation efficiency (CE) value $(0.50 \pm 0.04)$ among all derivatives, while the least functionalized $\mathrm{Hzd}$ derivative $(1.0 \mathrm{Hzd} / \beta \mathrm{CD})$ exhibits the second highest values of both $K_{a}$ $\left(760 \pm 220 \mathrm{M}^{-1}\right)$ and $\mathrm{CE}(0.16 \pm 0.04)$. Both phase solubility and ITC analyses suggest that increasing the number of functional groups on the $\beta C D$ ring limits the ability of dexamethasone to form an inclusion complex with $\beta C D$. The Dex- $\beta C D$ grafted polymer showed a similar stability constant and $\mathrm{CE}$ value to the $1.0 \mathrm{Hzd} / \beta \mathrm{CD}$ derivative used to prepare the graft polymer. The lowest functionalized $\mathrm{CM} / \beta C D$ derivative forms the largest aggregates when complexed with dexamethasone, while $\mathrm{Hzd} / \beta \mathrm{CD}$ derivatives form aggregates with sizes independent of functionalization. NTA data shows that dexamethasone does not drive aggregate formation in Dex- $\beta C D$ polymers.

Conclusion: $C M / \beta C D$ and $\mathrm{Hzd} / \beta C D$ derivatives exhibited similar complexation behaviors with dexamethasone, while polymer grafting resulted in no significant change in binding properties. These findings provide a framework for designing $\beta C D$ s as pharmaceutical excipients or drug binding sites in drug delivery vehicles.

\section{Drug Delivery \& Pharmaceutical Technology}

\section{Amino Acid Functionalized Nanodiamonds as Gene Delivery Vectors}

Saniya Alwani ${ }^{1}$, Jackson M. Chitanda ${ }^{2}$, Deborah Michel $^{1}$, Ronald E. Verrall ${ }^{2}$ and Ildiko Badea ${ }^{1}$. ${ }^{1}$ College of Pharmacy and Nutrition, ${ }^{2}$ Department of Chemistry, University of Saskatchewan, Saskatoon, SK, Canada

Purpose: This study is aimed at synthesizing amino acid (AA) functionalized nanodiamonds (NDs) as potential nanocarriers for gene delivery. Lysine-NDs are synthesized to introduce primary amines on the surface that can bind negatively charged genetic materials, and can also interact with the cell membrane to facilitate cellular uptake. Lysylhistidine-NDs were synthesized to create a $\mathrm{pH}$ sensitive delivery system which can allow endosomal escape and protect therapeutic genes against degradation.

Methods: Positively charged NDs were synthesized by covalent conjugation of lysine and histidine on the surface. Lysine and lysyl-histidine NDs (fNDs) were characterized by size and zeta potential measurements, infrared spectroscopy (IR) and thermogravimetry (TGA). Surface loadings of functionalized and non-functionalized NDs were also calculated. Interaction with serum proteins and stability in aqueous and tissue culture medium were analysed for fNDs by size and zeta potential measurements. Binding capacity of fNDs with pDNA and SiRNA at different weight ratios was 
analysed by gel electrophoresis. Uptake of fNDs in HeLa cells was evaluated using flow cytometry and scanning transmission X-ray microscopy (STXM).

Results: IR spectra of fNDs shows distinct amide peaks of N-H (stretch) at $\sim 3000 \mathrm{~cm}^{-1}, \mathrm{~N}-\mathrm{H}$ (bend) at $\sim 1490 \mathrm{~cm}^{-1}$ and $\mathrm{C}=\mathrm{O}$ (stretch) at $\sim 1650 \mathrm{~cm}^{-1}$ evidencing functionalization. Lysine NDs have a surface loading of $1.97 \mathrm{mmoles} / \mathrm{gm}$ which is higher than non-functionalized NDs (1.0 mmoles/gm). High surface loading refers to high binding capacity. Lysine functionalization was able to create highly uniform ND dispersion (PDI: 0.14) with a positive zeta potential of $+26.5 \mathrm{mV}$ and majority of particles having size of $\sim 50 \mathrm{~nm}$. Aqueous dispersion of lysine-NDs showed minimum aggregation and was found to remain stable over a period of 3 days. Lysine NDs were able to bind pDNA at 1:1 and SiRNA at 1:20 weight ratios. Flow cytometry results showed a fluorescence shift and an increase in relative fluorescence intensity for the cells treated with diamoplexes compared to untreated cells, indicating cellular association of NDs and delivery of SiRNA within cells.

Conclusion: Covalent functionalization of NDs with AAs can reduce aggregation and produce stable dispersion. Moreover, AA functionalized NDs are capable of binding genetic materials and shows a potential to protect and deliver therapeutic genes in mammalian cells.

\section{Trastuzumab Modified Targeted PLGA Nanoparticles for HER2 Positive Breast Cancer}

Sams Mohammad Anowar Sadat and Azita Haddadi College of Pharmacy and Nutrition, University of Saskatchewan, Saskatoon, SK, Canada

Background: Human epidermal growth factor receptor (HER2) is being overexpressed in around $30 \%$ of breast cancers, which is a fundamental biomarker for earlier stage pathogenesis and suitable for designing anticancer based targeted drug delivery system.

Objective: Main objective of this study is to design Trastuzumab modified HER2 receptor specific delivery system. The nanoparticles will then be investigated for physicochemical characterization and the effect of delivery systems against HER2 related breast cancer cells.

Methods: Docetaxel loaded poly-(D, L-lactic-coglycolide) (PLGA) nanoparticles were prepared following emulsification-solvent evaporation method. Trastuzumab was covalently attached with freeze-dried nanoparticles, which were pre-activated with the homo-bifunctional spacer, bissulfosuccinimidyl suberate (BS3). Size, zeta potential, polydispersity index, yield, drug loading, drug entrapment efficiency, and antibody quantification were considered as the key parameters to characterize the nanoparticle formulations.

Results: The size of nanoparticles without cryoprotectant was found to be from $130.40 \pm 2.30$ $\mathrm{nm}$ to $158.80 \pm 5.61 \mathrm{~nm}$ depending on the amount of drug and polymer ratio before freeze-drying, whereas it ranged from $344.03 \pm 56.14 \mathrm{~nm}$ to $877.50 \pm 287.30 \mathrm{~nm}$ after freeze-drying. To minimize the aggregation following the freeze-drying, formulations were cryopreserved using sucrose. Aggregation-free nanoparticles were obtained with an average size of $131.00 \pm 5.70 \mathrm{~nm}$ to $148.10 \pm 6.88$ $\mathrm{nm}$ and $279.5 \pm 6.90 \mathrm{~nm}$ to $342.90 \pm 11.43$ before and after freeze-drying, respectively. Drug encapsulation efficiencies and loadings of drug per $\mathrm{mg}$ of nanoparticles were found to be from $7.46 \pm 6.51 \%$ to $85.30 \pm 10.2 \%$ and from $0.51 \pm 0.03 \mu \mathrm{g}$ to $8.80 \pm 1.90$ $\mu \mathrm{g}$, respectively. In addition, amount of antibody loading on nanoparticles were also within the acceptable range.

Conclusion: The physicochemical characterization of drug loaded and antibody-modified nanoparticles were found to be within the desired range. This will further help to characterize and develop a targeted delivery system suitable for receptor mediated endocytosis.

57. The Impact of Implementation of the Canadian Regulatory Requirements on the Quality of Natural Health Products: The Glucosamine Case

$\underline{\text { Ali Aghazadeh-Habashi }}{ }^{1}$, John Duke ${ }^{2}$, Fakhreddin Jamali $^{1}$

${ }^{1}$ Faculty of Pharmacy and Pharmaceutical Sciences, and ${ }^{2}$ SLOWPOKE Reactor Facility, University of Alberta, Edmonton, Alberta, Canada

Purpose: We investigated whether the recent implementation of the regulatory requirements for the entry to the Canadian market of natural products has resulted in improved quality of the available glucosamine products.

Methods: Eleven available products, of which 8 had been tested in 2002 (7 had contained substantially 
lower than the label claim of the active ingredient), and a European pharmaceutical grade tablet were assayed for their glucosamine content. The potassium and sodium contents that are used to stabilize crystals of the solid dosage forms were also tested.

Results: Nine of the 11 Canadian products and the European tablet had more than $91 \%$ of the label claim of the active ingredient, hence, met the criterion. Two products contained 71 and $78 \%$ label claim. The electrolyte contents were very variable but constituted only a small fraction of the daily requirements.

Conclusion: Most tested glucosamine products passed the Health Canada requirements. This improvement is likely due to the publicity regarding the low quality of the products in the past and also a result, at least in part, of the introduction of the new regulatory requirements. The sub-standard quality of a few tested products is still of concern.

\begin{tabular}{|c|c|c|c|c|c|}
\hline \multirow{3}{*}{$\begin{array}{l}\text { Pro- } \\
\text { duct }\end{array}$} & \multirow{3}{*}{$\begin{array}{l}\text { Labeled } \\
\text { GlcN, mg } \\
500 / 5 \mathrm{~mL}\end{array}$} & \multicolumn{2}{|c|}{$\begin{array}{l}\% \text { of the labeled } \\
\text { GlcN content }\end{array}$} & \multirow{2}{*}{\multicolumn{2}{|c|}{$\begin{array}{l}\mathrm{K} \quad \mathrm{Na} \\
\mathrm{mg} / 1500 \mathrm{mg}^{\mathrm{b}}\end{array}$}} \\
\hline & & \multicolumn{2}{|c|}{$2002 \quad 2013^{\mathrm{a}}$} & & \\
\hline & & ND & 111(2.2) & ND & ND \\
\hline 2 & 500 & 108 & $105(2.0)$ & 361.7 & 104.3 \\
\hline 3 & 500 & 50 & $104(7.9)$ & 335.9 & 0.49 \\
\hline 4 & 500 & 55 & $104(7.2)$ & 330.3 & 0.56 \\
\hline 5 & $500 / 7.5 \mathrm{~mL}$ & ND & 104(2.9) & ND & ND \\
\hline 6 & 500 & 65 & $101(2.4)$ & 316.0 & 4.18 \\
\hline 7 & 750 & ND & $101(2.1)$ & 369.9 & 0.49 \\
\hline 8 & 500 & 60 & $94(2.2)$ & 326.2 & 0.29 \\
\hline 9 & 500 & 66 & $91(2.6)$ & 324.6 & 0.04 \\
\hline 10 & 1500 & 42 & $78(2.2)$ & 251.5 & 2.04 \\
\hline 11 & 500 & 55 & $71(1.0)$ & 256.5 & 0.05 \\
\hline $\begin{array}{l}\text { Rott } \\
a^{c}\end{array}$ & 250 & ND & 94 (1.1) & $\leq 17.9$ & 174.8 \\
\hline \multicolumn{6}{|c|}{$\begin{array}{l}{ }^{\mathrm{a}} \text { Data presented as mean (SD), } \mathrm{n}=3 / \text { product; }{ }^{\mathrm{b}} 1500 \mathrm{mg} \text { is the } \\
\text { common daily dose; }{ }^{\mathrm{c}} \text { European pharmaceutical product; ND, not } \\
\text { determined. }\end{array}$} \\
\hline
\end{tabular}

\section{In Silico Modeling (Gastroplus) to Overcome Pharmacogenomic Variation in Human Using Different Dextromethorphan Dosage Forms}

Muhammad Sarfraz, May Almukainzi and Raimar Löbenberg

Faculty of Pharmacy and Pharmaceutical Sciences, University of Alberta, Edmonton, Alberta, Canada

Purpose: Physiological based pharmacokinetic in silico modeling has become an integral tool in formulation development for Quality by Design
(QbD) approach The purpose of this study was to investigate how modeling can assist the formulation scientist in developing a controlled release dosage forms for drugs, which undergo extensive (EM) or poor metabolism (PM). Dextromethophan (DM) was selected as a model drug because its metabolism is sensitive to pharmacogenomic variations in different populations. Literature suggests that modifications in drug release kinetics might be able to over-come or reduce the differences between EM and PM.

Methods: A predictive model was generated using various physiological and pharmacokinetic parameters. Simulations were performed using a 30 mg (IR) tablet model with or without coadministration of quinidine as enzyme inhibitor. After validation of the predictive model simulations were performed with zero order release tablets $\left(F_{1}\right)$, first order sustained release tablets $\left(\mathrm{F}_{2}\right)$, an immediate release $(35 \%$ in $30 \mathrm{~min}$ ) followed by the zero order release tablets $\left(\mathrm{F}_{3}\right)$, an immediate release $(70 \%$ in $30 \mathrm{~min})$ followed by zero order release tablets $\left(\mathrm{F}_{4}\right)$ and an immediate release $(50 \%$ in 30 min) followed by pulse release of $50 \%$ drug at 2hours $\left(\mathrm{F}_{5}\right)$.

Results: There was a significant difference in the drug plasma profiles between EM and PM in all simulations. The IR formulation showed a larger $\mathrm{C}_{\max }$, longer $\mathrm{t}_{\max }$, and larger $\mathrm{AUC}_{0-24}$ in $\mathrm{PM}$ in comparison to EM. The co-administration of quinidine showed a gradual increase in drug plasma profile with increasing doses, however the effect was more pronounced in EM than in PM. $\mathrm{C}_{\max }$, and $t_{\max }$ varied depending on the formulation $\left(F_{1}-F_{5}\right)$ however, the $\mathrm{AUC}_{0-24}$ was comparable within the EM or PM population. The study showed that release profiles were able to alter the drug plasma profiles but did not overcome the difference in the observed drug plasma profiles in EM and PM

Conclusion: In silico modeling was able to predict the drug plasma profiles of different dosage forms. This can assist the formulation scientist to optimize the release properties of a drug from a dosage form to reach a desired drug plasma profile. However, study showed that drug delivery cannot address the differences between EM and PM which were due to pharmacogenomic variations. 


\section{Validation of a Molecular Dynamic Model: PEGylated Liposome Surface Interacting with Salt Ions of the Bloodstream}

$\underline{\text { Kevin Plourde }^{1}}$, Warren Viricel ${ }^{1}$, Alex Bunker ${ }^{2}$, Jeanne Leblond Chain ${ }^{1}$

${ }^{1}$ Faculty of Pharmacy, University of Montreal, Montreal, QC; ${ }^{2}$ Faculty of Pharmacy, University of Helsinki, Helsinki, Finland

Purpose: PEGylation of liposome is an efficient strategy to prolong bloodstream lifetime of these carriers, to decrease off-target toxicity of their drug content and improve drug accumulation in specific tissues. Even though these effects have been demonstrated, it isn't well understood how PEG molecules ensure these properties at the surface of liposomes. A molecular dynamic simulation has studied the effect of salt ions from the bloodstream on the surface of PEGylated lipid bilayer. It was found that smaller ions, such as sodium ion, could penetrate and accumulate more easily in a denser PEG corona, compared to bigger ions, such as chloride, calcium and potassium. In this work, we intended to confirm experimentally this behavior of liposomes, grafted with different density of PEG, in the presence of different concentrations and nature of salts.

Methods: Liposomes of DSPC were prepared with increasing molar ratios (0 to $10 \%)$ of DSPEPEG2000 using the hydration of a dry lipid film method. Liposomes were incubated in salt solutions $\left(\mathrm{NaCl}, \mathrm{CaCl}_{2}, \mathrm{KCl}\right.$ and $\left.\mathrm{MgCl}_{2}\right)$ at different concentrations (from 1.25 to $15 \mathrm{mM}$ salt solutions), and their surface potential was measured by DLSELS.

Results: Potential zeta of liposomes is increasing upon addition of increasing concentrations of $\mathrm{NaCl}$. This effect is not observed without PEG $(\Delta \zeta=2.7 \pm$ $0.7 \mathrm{mv}$ ), and is more obvious with increasing amount of PEG incorporated in liposomes $(\Delta \zeta=$ $18.1 \pm 2.2 \mathrm{mv}$, for $10 \% \mathrm{PEG})$. This trend is not confirmed in the presence of $\mathrm{CaCl}_{2}(\Delta \zeta=1.6 \pm 1.8$ $\mathrm{mv}$, for $10 \% \mathrm{PEG})$, but was observed in the presence of $\mathrm{KCl}(\Delta \zeta=22.1 \pm 2.6$, for $10 \%$ PEG $)$. These results suggest that sodium ions can be more easily trapped in the PEG corona than chloride ions and calcium ions. However, our results showed that these properties may be related to the monovalency (or hydration) more than the size of the ion, since potassium induced the same behaviour as sodium.

Conclusion: The hypothesis suggested by molecular dynamics was examined and refined using zeta potential measurements of PEGylated liposomes. Together, both techniques helped to clarify the role of PEG corona on liposome behaviour.

\section{Synthesis, Preparation, and Characterization of Polymeric Nanocapsules of Nifuroxazide Analogues}

${ }^{1}$ Michael Jefferson Amorim de Oliveira, ${ }^{2}$ Lívia Palmerston Mendes, ${ }^{2}$ Eliana Martins Lima, ${ }^{3}$ Luiz Alexandre Marques Wiirzler, ${ }^{1}$ Leoberto Costa Tavares

${ }^{1}$ Laboratory of Drug Design and Development, Faculty of Pharmaceutical Sciences, University of São Paulo, SP, Brazil; ${ }^{2}$ Laboratory of Pharmaceutical Nanotechnology and Drug Delivery Systems, Federal University of Goiás, GO, Brazil; ${ }^{3}$ Laboratory of Inflammatory Response, State University of Maringá, PR, Brazil

Purpose: Neglected diseases, especially Chagas disease need new treatment options. With the advent of nanotechnology, it is possible to improve properties such as solubility and permeability of drugs and new molecular entities (NME). More than 20 nifuroxazide analogues were submitted to in vitro bioassays against epimastigote form of Trypanosoma cruzi followed by studies of structure activity relationship (SAR). Among the most effective analogs, the Chlorine and Butyl replaced stood out. This study aimed to synthetize $p$-chloro and $p$-Butyl 5-nitro-2-furfuryliden (Figure 1) which were the most active against T. cruzi, and preparation of polymeric nanocapsules, using interfacial deposition of the preformed polymer (nanoprecipitation).

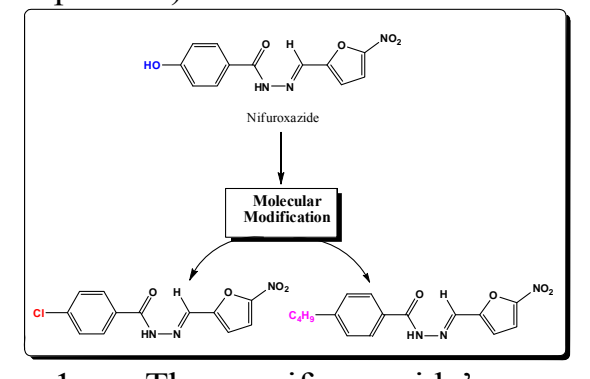

Figure 1. The nifuroxazide's molecular modifications and the analogs $p$-chloro and $p$-Butyl 5-nitro-2-furfuryliden.

Methods: Derivatives $p$-chloro and p-butyl 5substituted nitrofuran were synthesized by means of esterification and ammonolysis reactions in two steps; compounds underwent recrystallization by 
water. A characterization and structure determination was performed by determination of melting range, NMR with ${ }^{13} \mathrm{C}$ and ${ }^{1} \mathrm{H}$, and elemental analysis. Polymeric nanocapsules containing the compounds were obtained by interfacial deposition of preformed polymers. Physical-chemical and morphological characteristics were characterized, including size and size distribution.

Results: The two reactions yielded greater than $90 \%$ of $p$-chloro and $p$-butyl 5 -substituted nitrofuran. The melting range, NMR with $\mathrm{C}^{13}$ and ${ }^{1} \mathrm{H}$, and elemental analysis proved it possible to confirm the identity and purity of the synthesized compounds. Polymeric nanocapsules have indicated results of zeta potential, particle size distribution and polydispersity index characterizing good formation of nanoparticles. However, entrapment efficiency (EE\%) for both compounds in the nanoparticles was approximately $10 \%$.

Conclusion: The compounds were synthetized, purified, and characterized. The polymeric nanocapsules were developed. In this case polymeric nanocapsules presented a low $\mathrm{EE} \%$ and thus requires new studies.

\section{A Microinjection Device for Administering In Situ-Gelling Hydrogels for Ophthalmic Drug Delivery}

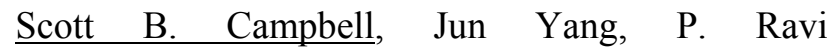
Selvaganapathy, and Todd Hoare

Purpose: Intraocular injections are routinely used to treat several posterior segment diseases, such as agerelated macular degeneration, diabetic retinopathy, and retinitis due to glaucoma. However, the frequency of injections required for these treatments greatly increases the risk of complications over time. In situ-gelling hydrogels that crosslink upon injection via a rapid chemical reaction between two functionalized polymers offer a potential solution, as they can facilitate sustained drug release kinetics over longer periods of time while remaining transparent and degradable. However, small volumes of each reactive material $(1-10 \mu \mathrm{L})$ must be injected to assess the in vivo capabilities of hydrogels in mouse and human eyes. While this is a simple process for single component systems, no suitable system exists for the administration of in situ-gelling hydrogels at such low volumes.

Methods: A microinjector has been developed that is capable of mixing two reactive polymers and controllably, rapidly, and precisely injecting volumes of 1-10 $\mu \mathrm{L}$ through a narrow gauge needle suitable for ophthalmic applications. The device design consists of a double barrel syringe connected to two separate inlets of a microfluidic chip, a serpentine mixing channel with herringbone mixing grooves, and a volume control reservoir with a oneway valve connected to second syringe that pushes the mixed polymer solutions out the needle and into the eye.

Results: With the volume control chamber and the one-way flow valve, the device is able to eject hydrogel droplets with controlled volumes $(\sim \pm 10 \%)$ in the range of interest $(1-10 \mu \mathrm{L})$ via an entirely handheld operation, requiring no additional equipment. The reactive polymer solutions are fully mixed after a short distance in the mixing channel, and hydrogel droplets can be formed after injection through $33 \mathrm{G}$ needles or capillaries into various materials, including bovine vitreous humour at $37^{\circ} \mathrm{C}$. The devices have been designed for ease of use for in vivo mouse eye injections, the results of which will be discussed.

Conclusions: A microinjection device has been developed that is capable of injecting small amounts of in situ-gelling hydrogel precursors to clinically exploit the favorable properties of injectable hydrogels in vivo as ocular drug delivery materials.

\section{Characterization of a Novel Surface-coated Matrix Intravaginal Ring Device for the Sustained Release of Hydroxychloroquine}

$\underline{\text { Yufei Chen }}^{1}$, Keith R. Fowke ${ }^{2,3}$, and Emmanuel A. $\mathrm{Ho}^{1}$

${ }^{1}$ Laboratory for Drug Delivery and Biomaterials, Faculty of Pharmacy, University of Manitoba, Winnipeg, Manitoba; ${ }^{2}$ Department of Medical Microbiology and Infectious Diseases, ${ }^{3}$ Department of Community Health Sciences, Faculty of Medicine, University of Manitoba, Winnipeg, Manitoba, Canada

Purpose: The aim of the current study is to develop and characterize a novel intravaginal ring (IVR) medical device for the sustained delivery ( $>14$ days) of hydroxychloroquine (HCQ) as a strategy for preventing male-to-female transmission of HIV.

Methods: $2 \%$ or $4 \%(\mathrm{w} / \mathrm{w})$ HCQ-loaded IVRs were fabricated from polyurethane through hot-melt injection molding. $100 \mathrm{mg}$ of IVR segments either non-coated or coated with $10 \%$ polyvinylpyrrolidone 
(PVP) or 5\% poly(vinyl alcohol) (PVA) were used for in vitro release studies. End-capped segments were incubated in $5 \mathrm{~mL} 25 \mathrm{mM}$ sodium acetate buffer at $\mathrm{pH}$ 4. HCQ release was quantitated using an RP-HPLC method. Accelerated stability studies were carried out at room temperature (RT) or at $40^{\circ} \mathrm{C} / 75 \%$ relative humidity (RH) in a climate chamber. Samples were collected and remaining HCQ was extracted and quantified. In vitro biocompatibility of drug-free IVR segments were evaluated in human vaginal and ectocervical epithelial cell lines. Cell viability and proinflammatory cytokine (IL-1 $\beta$, IL-6, and IL-8) production were evaluated via MTS assay and ELISA, respectively. The impact of drug-free IVR segments on individual cell division and proliferation was carried out using a colony formation assay.

Results: IVR segments used in the proposed study had dimensions of $25 \times 5 \mathrm{~mm}$ (outer diameter $\mathrm{x}$ cross-sectional diameter). Release studies showed a sustained HCQ release over 18 days. During the study period, $83.83 \pm 3.57 \%$ and $85.51 \pm 1.81 \%$ of loaded HCQ was released from $2 \%$ and $4 \%(\mathrm{w} / \mathrm{w})$ IVR segments, respectively. It was estimated that approximately $1533.53 \mathrm{mg}$ and $3131.81 \mathrm{mg}$ of HCQ could be released from a $2 \%$ and $4 \%(\mathrm{w} / \mathrm{w})$ full size human IVR (55 x $5 \mathrm{~mm}$, outer diameter x crosssectional diameter) within 18 days, respectively. IVRs coated with PVP or PVA exhibited a reduced burst release on the first day $(34.63 \pm 2.39 \%$ and $25.36 \pm 2.62 \%$, respectively). Stability studies demonstrated that HCQ was stable in both coated and non-coated IVRs stored at RT or at $40^{\circ} \mathrm{C} / 75 \%$ RH. No cytotoxicity or significant cytokine production was observed when cells were treated with drug-free IVR segments for 30 days.

Conclusions: Our research group is the first to fabricate a surface-coated IVR that can provide sustained release of HCQ for 18 days. This IVR system is non-cytotoxic and may be a suitable platform for the prevention of HIV transmission and other sexually transmitted diseases.

\section{Drug Release System Based on an Inorganic Phosphate Hydrogel with Extreme Thixotropic Behavior}

Marco Laurenti ${ }^{1}$, Jake Barralet ${ }^{1,2}$, and Faleh Tamimi ${ }^{1}$ ${ }^{1}$ Faculty of Dentistry, McGill University, Montreal, Quebec; ${ }^{2}$ Department of Surgery, Faculty of Medicine, McGill University, Montreal, Quebec, Canada

Purpose: A novel inorganic hydrogel has been developed based on phosphate and magnesium. The inorganic gel combines key properties such as stability, biocompatibility, bioresorption, and injectability. The aim of this study was to investigate the ability of the gel for controlled drug release of Doxycycline.

Methods: The inorganic gel has been characterized using different techniques: X-Ray diffraction patterns, Transmission Electron Microscopy using a FEI Tecnai T12 working at $120 \mathrm{kV}$, rheology using a TA AR2000, and the drug release has been study using UV-Vis spectroscopy. Cell viability test has been performed using human fibroblast cells to assess the biocompatibility of the novel inorganic hydrogel. Doxycycline is incorporated inside the gel prior to begin the release into a freshly prepared PBS solution at $37^{\circ} \mathrm{C}$ using a dialysis kit.

Results: The inorganic phosphate hydrogel is produced by mixing aqueous solutions containing specific concentrations of ions at room temperature. The gel forms within minutes and is stable at neutral $\mathrm{pH}$ for a long period of time. Rheological analysis revealed that the gel is thixotropic, which makes it very useful for applications requiring minimally invasive injection such as the treatment of periimplant infection.

Interestingly the liquid-to-gel transitions occur within less than 6 seconds of the induction and removal of shear stress, making this extremely highspeed transition very uncommon in hydrogels ${ }^{4}$. The cell viability performed on human fibroblast shows a full biocompatibility of this novel biomaterial.

Doxycycline has been used to assess the drug release kinetics of the drug from the hydrogel, and the release kinetics follows the model described by the Korsmeyer-Peppas equation $M / M t=k t n$. The kinetics presents two different regions: 1) in the first region the release follows the super Case II transport, and 2) the release is regulated by Fickian diffusion.

Conclusion: A new inorganic phosphate hydrogel has been synthesized, characterized, and assessed for 
controlled drug release. Our system shows a good controlled release of Doxycycline following two different mechanisms: 1) super Case II transport, and 2) Fickian release. To the best of our knowledge these properties have never been observed in one single material and they open a wide range of biomedical applications for the new inorganic phosphate gel for in vivo study.

\section{Scavenger Receptor Class B Type I (SR-B1) Expression Levels Modulate CPX-351 Uptake within Cancer Cells}

Jenny Hanbi Kim ${ }^{1}$, Paul Tardi ${ }^{2}$, Edward S. Shin ${ }^{1}$, Lawrence Mayer $^{2}$ and Kishor M. Wasan ${ }^{1}$

${ }^{1}$ Faculty of Pharmaceutical Sciences, University of British Columbia, Vancouver, BC; ${ }^{2}$ Celator Pharmaceuticals Inc., Vancouver, BC, Canada

Purpose: The purpose of this study was to assess the role of SR-BI on the uptake of CPX-351, a novel liposomal formulation containing a 5:1 mole ratio of cytarabine and daunorubicin, within cancer cells expressing elevated levels of SR-B1.

Methods: Small interfering RNA was used to down regulate the protein expression of SR-B1 within C42 castration-resistant prostate cancer (CPRC) cells which we have previously shown to express elevated SR-B1 levels. The inhibition of protein expression and decrease in activity were confirmed by western blot and HDL-cholesterol cellular influx analysis, respectively. The effect of down-regulation of SR-BI on CPX-351 liposome uptake was measured by the ability of CPX-351 to emit fluorescence at 480/595nm when fluorescently-tagged daunorubicin content is processed and released inside the cells.

Results: The greatest percentage of SR-BI protein down regulation $(75 \%$ compared to non-treated controls; $\mathrm{n}=3 ; \mathrm{p}<0.05$ vs. non-treated control) was observed after 5 hours of transfection using $10 \mathrm{nM}$ siRNA and $1 \% \mathrm{v} / \mathrm{v}$ RNAiMAX;). Statistically significant decreases in the cellular uptake of CPX351 were observed across a 20-200nM daunorubicin concentration range with the greatest reduction of $43 \%$ at $100 \mathrm{nM}$ of Daunorubicin in SR-BI down regulated cells compared to non-treated control cells $(\mathrm{n}=3 ; \mathrm{p}<0.05$ vs. untreated controls).

Conclusion: These preliminary studies suggest that SR-BI may play a role in the uptake of CPX-351 liposomes into cancer cells and could represent a new potential avenue to target therapeutic agents to CRPC.
Acknowledgement: Funding provided by the Celator Pharmaceuticals Inc.

\section{Targeting CD205 Receptor as an Approach to Develop a Cancer Vaccine}

Sheikh Tasnim Jahan and Azita Haddadi

College of Pharmacy and Nutrition, University of Saskatchewan, Saskatoon, SK, Canada

Background: Stimulation of a patient's immune system to fight cancer is the underlying mechanism of immunotherapy. Cancer immunotherapy manipulates the dendritic cells to identify cancer antigens in the immunosuppressive microenvironment. This vaccination strategy based on nanoparticulate drug delivery system has the potential to treat cancer through packaging of therapeutic cargoes and delivering them to target immune cells (dendritic cells, DCs). FDA approved poly-(D, L-lactic-co-glycolide) is particularly competent due to its low immunogenicity, low toxicity, biocompatibility and biodegradability.

Purpose: i) To develop structurally modified nanoparticle delivery system with anti-CD-205 antibody, ii) Physicochemical characterization of nanoparticles, iii) Assessing the targeting efficiency of PLGA nanoparticles by DC uptake studies and iv) Evaluating the influence of modified PLGA nanoparticles in enhancing DC stimulatory immune responses in dendritic cell lines.

Methods: PLGA nanoparticles were formulated by $\mathrm{O} / \mathrm{W}$ emulsification-solvent evaporation technique. Ovalbumin (OVA) loaded nanoparticles were prepared by $\mathrm{W} / \mathrm{O} / \mathrm{W}$ emulsification-solvent evaporation method. The DC targeting ligand was attached to the nanoparticle surface through covalent binding in the presence of spacer molecule (bissulfosuccinimidyl suberate) and physical adsorption method. Formulations were evaluated with respect to particle size, polydispersity index, zeta potential, surface display, cytotoxicity assay, DC uptake study and DC maturation study. Finally there were several groups of nanoparticle formulation as: Empty, antibody modified, fluorescent dye loaded, antigen and/or adjuvant loaded nanoparticles.

Results: The PLGA nanoparticles had desirable size, polydispersity index, zeta potential, surface display and safety profile when cryoprotectant was used. Formulations having cryoprotectant provided aggregation free colloidal dispersion. Flow cytometry experiment with fluorescent dye loaded 
nanoparticles showed that the ligand modified nanoparticles were better uptaken by DCs when compared with the non-modified nanoparticles. Maturation study of DCs demonstrates that ligand modified OVA-adjuvant loaded nanoparticles performed higher upregulation of maturation markers.

Conclusion: Therefore, our goal is to design a structurally concealed nanoparticle vaccine delivery system, which will be able to provoke the DCs to effectively present the epitopes to obtain antigen specific response in a similar but efficient manner antigen does in-vivo.

\section{Application of Super Fine Powder, Special Low Viscosity Grade of Hydroxypropyl Cellulose (HPC-SSL-SFP) as a Tablet Hardness Enhancer for Hydrophilic Matrix Formulation}

Ryan Cheng $^{1}$, Catherine Cote $^{1}$, Kenji Sugisawa ${ }^{2}$, Shinichiro Tsue ${ }^{2}$, Takeshi Shimotori ${ }^{2}$

${ }^{1}$ Nisso America Inc., 88 Pine Street, New York, NY 10005; ${ }^{2}$ Nippon Soda Co., Ltd, Shin-Ohtemachi Bldg., 2-1, Ohtemachi 2-Chome, Chiyoda-ku, Tokyo 100-8165, Japan

Purpose: Super fine powder, special low viscosity hydroxypropyl cellulose (HPC-SSL-SFP) is a newly developed dry binder which can provide excellent tablet properties at low usage level. In this study, we evaluated the performance of HPC-SSL-SFP as a tablet hardness enhancer for hypromellose (HPMC) matrix formulation by direct compression (DC) method using theophylline as a model drug.

Methods: The powdered mixtures for three tablet formulations were prepared by dry-mixing theophylline (50\%), HPMC (30\%), HPC-SSL-SFP (x), MCC (19-x), silica (0.5\%), and magnesium stearate $(0.5 \%)$, where $\mathrm{x}=0 \%$ (control), $5 \%, 10 \%$. Tablets were compressed at $10 \mathrm{kN}$ by DC, and the tablet weight and diameter were $200 \mathrm{mg}$ and $8 \mathrm{~mm}$ respectively. Tablet hardness was measured and the release of theophylline was monitored by drug molar concentration in water using a UV spectrophotometer.

Results: The hardness of the tablet with $5 \%$ and $10 \%$ of HPC-SSL-SFP was $18.1 \mathrm{kgf}$ and $21.1 \mathrm{kgf}$ respectively while the tablet without HPC-SSL-SFP was $11.9 \mathrm{kgf}$. The results showed tablet hardness could be improved remarkably by the addition of HPC-SSL-SFP. Moreover, the results showed drug release was not affected by the addition of HPCSSL-SFP, since it is a water-soluble polymer with super fine particle size and low viscosity that can be dispersed and dissolved into water quickly.

Conclusion: HPC-SSL-SFP was found to be an effective tablet hardness enhancer without changing the drug release profile for hydrophilic matrix formulation prepared by DC method using HPMC.

\section{Application of Hydroxypropyl Cellulose to Solubility Enhancement of Poorly Soluble Drugs}

Ryan Cheng $^{1}$, Catherine Cote ${ }^{1}$, Kenji Sugisawa ${ }^{2}$, Shinichiro Tsue ${ }^{2}$, Ashish Sarode ${ }^{3}$, David R. Worthen ${ }^{3}$

${ }^{1}$ Nisso America Inc., 88 Pine Street, New York, NY; ${ }^{2}$ Nippon Soda Co., Ltd, Shin-Ohtemachi Bldg., 2-1, Ohtemachi 2-Chome, Chiyoda-ku, Tokyo, Japan; ${ }^{3}$ Department of Biomedical and Pharmaceutical Sciences, College of Pharmacy, and Department of Chemical Engineering, College of Engineering, University of Rhode Island, Kingston, RI

Purpose: Low viscosity grades of hydroxypropyl cellulose (HPC) are versatile pharmaceutical excipients which can be used for solubility enhancement of poorly soluble drugs. In this study, the utility of HPC-SL and HPC-SSL polymers was assessed as hot melt extrusion (HME) excipients for solubility enhancement of carbamazepine (CBZ) and phenytoin (PHT), two BCS Class II drugs. Processibility and dissolution enhancement efficiency of solid dispersions (SDs) manufactured by hot melt mixing (HMM) of these drugs with the low viscosity HPC polymers was evaluated. Solvent evaporation (SE) and ball milling (BM) were investigated for solubility enhancement of very high melting drug PHT.

Methods: Physical Mixtures (PMs) were prepared by mixing each drug with HPC-SL and HPC-SSL at a 25:75 drug:polymer ratio $(\mathrm{w} / \mathrm{w})$. The blended PM were compacted, milled and sieved between US mesh nos. 40 and $60(420-250 \mu \mathrm{m})$. PMs were hot melt mixed, ball milled, and solvent evaporated in vacuo to obtain the respective amorphous SDs. Amorphous transformation was investigated using differential scanning calorimetry (DSC) and polarized light microscopy (PLM). Milled SDs were filled into capsules for dissolution studies.

Results: The melting point depression of CBZ was observed in its PMs formulated with HPC-SL and 
HPC-SSL but not detected in HMMs indicating that crystalline drug was converted into a state of amorphous. The dissolution rate and extent of CBZ was found to be significantly higher in the case of HMM than the PM, owing to the amorphous conversion of the drug by the HMM process. The dissolution rate and extent of PHT was only slightly enhanced by HMM. Since the melting point of PHT is very high $\left(290-300{ }^{\circ} \mathrm{C}\right)$, it was estimated that the drug could not be mixed and dispersed thoroughly during the HMM process at the operating temperature of $180{ }^{\circ} \mathrm{C}$ to provide effective small drug particle sizes. Using $\mathrm{SE}$ and $\mathrm{BM}$, the enhancement in dissolution rate of PHT with HPCSSL significantly improved. The better performance of SE and BM products is owed to better mixing and dispersing to manufacture smaller drug particle sizes compared to the HMM products.

Conclusion: HPC-SL and HPC-SSL could be used to convert $\mathrm{CBZ}$, which has a relatively high melting point, into a state of amorphous using HMM process and dissolution could be improved significantly. Although dissolution rate of PHT, which has a very high melting point, could not be improved by HMM, it could be significantly improved by manufacturing its crystalline dispersions in HPC-SSL using BM and SE.

\section{Relationship between PEG Surface Coverage- density and the Physico-chemical Properties of Polymeric Nanoparticles}

\author{
Jean-Michel Rabanel $^{1,2}$, Patrice Hildgen ${ }^{1}$, Xavier \\ Banquy $^{2}$ \\ ${ }^{1}$ Laboratoire de Nanotechnologie pharmaceutique; \\ ${ }^{2}$ Chaire de recherche du Canada sur les matériaux \\ inspirés du vivant, Faculté de Pharmacie, Université \\ de Montréal, Montréal, QC, Canada
}

Purpose: Addition of a PEG layer to circulating drug carriers such as solid nanoparticles (NP) has been showed to be a key element to extend their circulation time and thus to increase drug efficacy. PEG efficacy is dependent on coverage-density, determined by quantification methods such as colorimetric, fluorescence detection or NMR. An important part of the NP optimization process is to establish a relationship between PEG coveragedensity and physical properties of NP. We choose to study the NP dispersion stability at physiological salts concentrations. Salt concentration is leading to aggregation of charged NP affecting their circulation time and biodistribution profile. Addition of a PEG layer is expected to stabilize these dispersions by steric repulsive forces in a coverage-density dependent manner.

Methods: Copolymerizations of $\mathrm{D}, \mathrm{L}$ dilactide in presence of variable molar ratio of Benzyl Glycidyl Ether were carried out by ring-opening polymerization. Alcohol pendant group were deprotected by catalytic hydrogenation. PEGs $(2 \mathrm{kD})$ were grafted by acylation to yield PEG- $g$-PLA. The polymers were characterized by GPC, FT-IR, and NMR. NPs were prepared by nanoprecipitation. After dialysis, NPs were kept in suspension at $4^{\circ} \mathrm{C}$. NPs prepared by nanoprecipitation in $\mathrm{D}_{2} \mathrm{O}$ were analyzed by ${ }^{1} \mathrm{H}$ NMR, and surface-bound PEG was quantified using an internal standard. Protein binding was studied by measuring the NP hydrodynamic diameters and zeta potential before and after incubation with BSA. Variations of NP sizes were measured, as a function of salt concentration using the Malvern Zetasizer automatic titration mode.

Results: Different polymer batches were synthesized to generate a library of PEG-g-PLA polymers. NP batch characterizations showed that PEG mass \% in polymer (in the range tested), did not affect significantly NP zeta potential. ${ }^{1} \mathrm{H}$ NMR quantitative analysis of PEG present at the NP surface showed a density above the brush regime threshold. BSA binding to NP did not affect their size, however, zeta potential was affected in the case of PEG-g-PLA polymers. Diblock polymer NP did not show any evidence of protein interactions. As expected, NP aggregation behavior was found to be dependent on PEG mass in polymeric material as well as PEG coverage-density.

Conclusion: PEG coverage-densities of NP prepared by nanoprecipitation, were correlated to protein absorption capabilities, salt stability and zeta potential. Critical coagulation concentration appears more sensitive than zeta potential to PEG coveragedensity variations. These observations may be of interest in the future development of drug polymeric nanocarriers. 


\section{Catechol-Chitosan/Genipin Mucoadhesive Hydrogel for Buccal Drug Delivery: An In Vivo Study}

$\underline{\text { Jinke Xu }}^{1}$, Satu Strandman ${ }^{2}$, Julian X.X. Zhu ${ }^{2}$, Jake Barralet $^{3}$ and Marta Cerruti ${ }^{1,3}$

${ }^{1}$ Department of Mining and Materials Engineering, McGill University, Montreal; ${ }^{2}$ Department of Chemistry, Université de Montréal, Montreal; ${ }^{3}$ Faculty of Dentistry, McGill University, Montreal, Canada.

Purpose: Drug administration via buccal mucosa is an attractive drug delivery strategy due to the advantages such as good patient compliance, prolonged localized drug effect, and avoidance of gastrointestinal drug metabolism and first-pass elimination. In our previous study, catecholchitosan/genipin (Cat-CS/GP) hydrogel system showed sustained release of lidocaine in PBS, and exhibited good mucoadhesion on porcine buccal mucosa in vitro. In this follow-up study, we applied the mucoadhesive hydrogel system to rabbit buccal mucosa to evaluate drug release in vivo. We also evaluated biocompatibility of this hydrogel by histological examination.

Methods: Catechol functional groups were covalently bonded to chitosan by EDC coupling method of chitosan and hydrocaffeic acid, with catechol content of $19 \%$. Hydrogel was prepared by crosslinking Cat-CS with GP (Cat19-CS/GP). Lidocaine was used as a model drug. A protective layer made in ethyl cellulose was prepared to prevent drug release to saliva. Lidocaine loaded Cat19-CS/GP hydrogel was adhered to rabbit buccal mucosa. Blood samples were collected at certain time points, followed by quantification of lidocaine concentration in the serum by LC-MS. CS crosslinked by GP hydrogel (CS/GP) was used as control. After the animals were sacrificed, buccal mucosal tissue in contact with the hydrogel was extracted for histological examination. Buccal mucosal tissue from the cheek that was not in contact with the hydrogel was also collected as control.

Results: Serum concentration of lidocaine is much higher if the drug is released from patches made of Cat19-CS than unmodified CS. Cat19-CS/GP hydrogels can provide sustained lidocaine release in vivo for at least three hours, while the concentration of lidocaine measured in the serum of the control group decreases significantly after 2 hours, reaching almost negligible values at the end of the experiment. This is because of the enhanced mucoadhesion of Cat19-CS/GP that provides more intimate contact with the mucosal surface than CS/GP. The histological examination on the buccal tissue in contact with the hydrogel shows no significant difference compared to the control, indicating good biocompatibility of the hydrogel system.

Conclusion: Catechol-modified CS hydrogels show higher mucoadhesion and lidocaine release compared to unmodified CS hydrogels when applied to rabbit buccal mucosa in-vivo. These hydrogels would allow for the delivery of sensitive proteins or nutraceuticals that cannot be mixed within the dry tablets currently used for buccal drug delivery. Additionally, they could be used in other applications where mucoadhesion is required, such as oral or intra-peritoneal drug delivery.

\section{Omega-3 Tablets Based on CarboxyMethyl Starch}

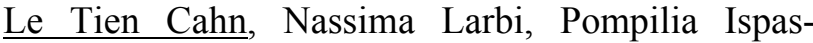
Szabo, Mircea Alexandru Mateescu

Département de chimie and Pharmaqam, Université du Québec à Montréal, Montreal, Quebec, Canada

Purpose: Omega-3 fatty acids are essential polyunsaturated fatty acids commercialized as softgel capsules or liquids. Pharmaceutical products prescribed for cardiovascular dysfunctions are ethyl esters semi-synthetic forms having certain disadvantages. Considering the increased interest in Omega-3 formulations able to preserve their natural composition and inner properties, the aim of this study was to introduce a new formulation of Omega3 stabilized with Carboxymethyl Starch (CMS) in powder form and ready-to-use for preparation of tablets.

Methods: Existing methods for Omega-3 encapsulation tend to generate preparations with high oily surface, low omega-3 content or poor stability limiting thus commercialization. This study proposes spray drying as procedure to obtain stable Omega-3/CMS/Gelatin powder preparations which were used for monolithic tablets preparation via direct compression. In vitro dissolution tests, antioxidant capacity after UV exposure were performed and correlated with structural data.

Results: CarboxyMethyl Starch can generate stable structures under powder or tablet form that can deliver Omega-3. The properties of these novel 
formulations result from the ability of CMS to form an inclusion complex with Omega-3 and generate a matrix able to control the Omega-3 release. Helical structure of starch represents a key-element affording stabilization of Omega-3 and its release in intestinal fluid. Gelatin adds further stabilization for the CMS-Omega-3 complex via polar interactions (model supported by structural analyses i.e. iodine test, FT-IR, X-ray). Better antioxidant capacity after exposure to UV light was found when compared to commercial liquid-filled capsules. Furthermore, CMS matrix affords mechanical stability and reduced fish smell with better intestinal absorption.

Conclusion: As monolithic tablets, the CMSOmega-3/G complexes can improve bioavailability by reducing the duration of gastric residence and floatability, favoring the intestinal delivery and increased compliance. In powder form, this novel complex can be used as food supplement or as excipient for the formulation of lipophilic compounds susceptible to oxidation (i.e. vitamins, antibiotics, antiviral and antitumoral agents). With better antioxidant capacity and lesser fish off-flavor, the novel omega-3 form has a lot of potential in nutraceutical and pharmaceutical fields. The novel CMS complexes can extend the area of CMS applicability as excipient for formulation of lipophilic bioactive agents i.e. increasing their solubility. Additional advantages are easy processing, low costs, patient compliance, versatility and high biocompatibility.

Acknowledgments: Support from NSERC (Discovery Grant) is gratefully acknowledged.

\section{Inkjet Printing of Therapeutic Warfarin Doses, How Printing can Address Clinical Needs of Patients}

Mustafa Alomari ${ }^{1}$, Joseph Standing $^{2}$, Abdul Basit ${ }^{1}$, Simon Gaisford ${ }^{1}$

${ }^{1}$ UCL School of Pharmacy, London, UK; ${ }^{2}$ UCL Institute of Child Health, London, UK

Purpose: Warfarin is the most commonly used anticoagulant in preventing thrombotic events. Patients walk a tightrope between bleeding and clotting, as numerous factors can tip the balance of anticoagulant control.

Personalising the dose can help achieve the required therapeutic level of the medicine. Thermal Inkjet Technology (TIJ) delivers precisely controlled volumes of ink solution onto edible substrates.
However, TIJ suffers from low volume deposition (and thereby dose) and lack of linear control of printed dose.

Methods: A commercial TIJ HP printer was modified to allow finer dose control and enable deposition of solutions onto a smaller area of substrate.

GMP-approved films were used as substrate on which warfarin was deposited. Ink solution was aqueous warfarin solution. Printed solutions were deposited on $0.8 \mathrm{~cm}^{2}$ of oral film. The volume of the deposited warfarin solution was controlled by a series of selected experimental y-values.

Results: Warfarin was successfully deposited onto the oral films. Clinically effective doses of up to 2 $\mathrm{mg}$ were achieved per single pass of the modified inkjet printer.Dose control was achieved, with low content uniformity variation within pharmacopoeial limits ( $\mathrm{RSD}<3 \%)$ and excellent linearity $\left(\mathrm{R}^{2}=0.999\right)$ as shown in figure (1).Films released the full dose of the medicine upon dissolution of the oral film alone and when enclosed in capsule shell.

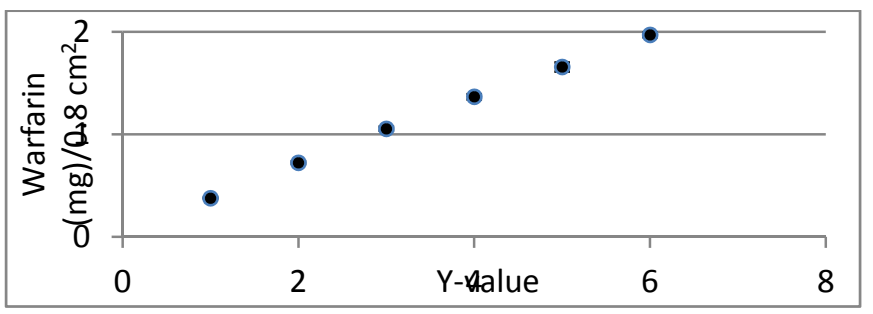

Figure (1): plot of y-values versus warfarin content of $0.8 \mathrm{~cm}^{2}$ film $(\mathrm{n}=3)$

\section{Conclusion:}

Innovation with currently available technology can help tackle medicine delivery challenges

\section{Greyscale Inkjet Printing of Personalised Levothyroxine Doses and Optical Dose Verification}

Mustafa Alomari ${ }^{1}$, Joseph Standing ${ }^{2}$, Abdul Basit ${ }^{1}$, Simon Gaisford ${ }^{1}$

${ }^{1}$ UCL School of Pharmacy, London, UK; ${ }^{2} \mathrm{UCL}$ Institute of Child Health, London, UK

Purpose: There is a need to flexibly titrate the dose of narrow therapeutic index medicine levothyroxine (T4) in special hypothyroid populations (pregnant women, children and the elderly). This flexibility is lacking since $\mathrm{T} 4$ is available in a few fixed-dose tablets. The need for personalised dosing of T4 may 
be addressed by the capacity of Thermal Inkjet technology (TIJ) to dispense flexible and precise volumes of solutions onto edible substrates.

Desktop TIJ printers control volume of deposited black ink solution by means of the darkness of the grey shade being printed. If the black ink is replaced with T4 solution, it may be possible to tailor the dose produced onto a substrate by means of choosing the desired grey shade of the square. In clinical settings, medicines being dispensed must be verified for requested dose. Verification of the printed dose may be done non-destructively using optical scanner which measures scanned shade (SS) of the colour they impart on the substrate.

Methods: On an HP desktop printer, a series of greyscale-shaded squares (shades: $0 \%$ "white" to $100 \%$ "black", area $1 \mathrm{~cm}^{2}$ ) in Microsoft Word document template (requested shade, RS) were entered and printed doses (PD) on the target substrate were produced.

T4 solution was pipetted into a modified TIJ cartridge which was inserted into the printer. Acetate was used as a model substrate due to ease of optical scanning. Medicine on printed pieces was analysed with HPLC-UV to obtain PD. HP Optical scanner was used to scan the printed acetates. ImageJ software was used to calculate SS

Results: A printed therapeutic T4 range of 10-80 $\mu \mathrm{g} / \mathrm{cm}^{2}$ was achieved. The relationship of PD to RS was found to be exponential $\left(r^{2}=0.994\right)$ rather than linear. Good exponential correlation $\left(r^{2}=0.95\right)$ between the RS and the SS was detected, suggesting encouraging results for the optical dose verification technique used.

Conclusions: T4 can be deposited in therapeutic doses using TIJ. Dose can be flexibly and precisely controlled using different shades of grey as an input to the printer. Optical dose verification has potential for improvement as a quality control test for printed doses.

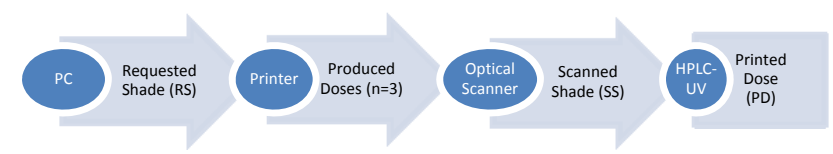

\section{Challenges and Opportunities to use Biowaivers to Compare Generics in China}

Jieyu Zuo ${ }^{1}$, Yuan Gao ${ }^{1}$, Nadia Bou-Chacra ${ }^{2}$, Raimar Löbenberg ${ }^{1, *}$

1. Faculty of Pharmacy and Pharmaceutical

Sciences, University of Alberta, Edmonton, Alberta,

Canada; Faculty of Pharmaceutical Sciences,

University of Sao Paulo, Sao Paulo, SP, Brazil

Purpose: Biowaivers for class I drugs according to the biopharmaceutics classification system (BCS) were first introduced in 2000. In vitro dissolution testing of a reference product and a generic can be used to document bioequivalence. Traditionally, bioequivalence between products was established via in vivo clinical trials, which are more expensive and time consuming. This study aimed to investigate the use of biowaivers to compare the in vitro dissolution behavior of two BCS class I drugs, amoxicillin and metronidazole, which are marketed in China.

Method: Three 250-mg and two 500-mg amoxicillin capsules and four metronidazole tablet products were tested. The dissolution behavior was measured in simulated gastric fluid (SGF), $\mathrm{pH} 4.5$ buffer and simulated intestinal fluid (SIF). A Varian dissolution tester VK 7020 was used with an auto sampler. $1 \mathrm{~mL}$ samples were taken at 10,15,20,30, 45 and 60 minutes and analyzed by HPLC at $219 \mathrm{~nm}$ and 228 $\mathrm{nm}$ for amoxicillin and metronidazole respectively. The dissolution behaviors of these products were compared by similarity factor $f_{2}$ to establish bioequivalence between the tested products.

Results: Identifying a reference product for the comparison on the Chinese domestic market was impossible. This is different in the US or Canada where the Orange book or a Health Canada Database can be used to identify the reference listed drug. Our results showed that none of the amoxicillin products were in vitro similar to each other in all three media. However, all products were similar in SGF, but $f_{2}$ values were under the threshold value of 50 in at least one media. Three of the four metronidazole tablet formulations were found to be in vitro equivalent to each other when the same strengths were compared. However, one product failed the in vitro comparison in SGF and cannot be assumed to be bioequivalent.

Conclusion: The study showed that it is impossible to identify a reference product on the Chinese market. Establishing a nationally or globally accepted reference product may provide regulatory 
agencies with an efficient mechanism for approving high quality generics.

(Research previously published: Zuo J, Gao Y, BouChacra N, Löbenberg R. Challenges and Opportunities to Use Biowaivers to Compare Generics in China. AAPS PharmSciTech. In press.)

\section{Evaluation of the DDSolver Software Applications}

Jieyu Zuo ${ }^{1}$, Yuan $\mathrm{Gao}^{2}$, Nadia Bou-Chacra ${ }^{3}$, Raimar Löbenberg ${ }^{1, *}$

${ }^{1}$ Faculty of Pharmacy and Pharmaceutical Sciences, University of Alberta, Edmonton, AB, Canada; ${ }^{2}$ Department of Pharmacy, Changhai Hospital, Second Military Medical University, Shanghai, China; ${ }^{3}$ Faculty of Pharmaceutical Sciences, University of Sao Paulo, Sao Paulo, SP, Brazil

Purpose: When a new oral dosage form is developed, its dissolution behavior must be quantitatively analyzed. Dissolution analysis involves a comparison of the dissolution profiles and the application of mathematical models to describe the drug release pattern. This study aimed to assess the application of the DDSolver, an Excel add-in software package, which is designed to analyze data obtained from dissolution experiments.

Method: The first data used to evaluate the dissolution profiles were obtained from a dissolution study of three different 250-mg amoxicillin capsules purchased from China. The second data used to investigate drug release mechanism were from drug release of rifampicin from two groups of gelatin nanoparticles. One in the presence of trypsin (forced degradation), whereas another was measured in the absence of trypsin (normal release). DDSolver was compared to a manual calculation using Excel.

Results: The comparisons among three different products obtained similarity factors $\left(f_{2}\right)$ of 23.21 , 46.66 and 17.91 , indicating that these products are not in vitro similar to each other. The use of DDSolver to perform these comparison was simple and provided accurate results which was confirmed by a manual calculation. The drug release fitting to the Korsmeyer-Peppas model showed differences between the DDSolver $(\mathrm{n}=0.392)$ and manual calculation $(\mathrm{n}=0.437)$. The DDSolver program uses the nonlinear least-squares curve-fitting technique to estimate such parameters by fitting the nontransformed dissolution data to the model. Whereas the $n$ value for the Korsmeyer-Peppas model was obtained by plotting the data on a logarithmic scale according to the equation, and the $\mathrm{n}$ value was determined using the slope. This causes the differences between the two calculation methods.

Conclusion: Performing routine quantitative analysis proved to be much easier using the DDSolver program than manual calculations. The use of the DDSolver program reduced the calculation time and has the potential to omit calculation errors, thus making this software package a convenient tool for dissolution comparison. An in-depth knowledge of the model fitting used in DDSolver is needed to utilize this software accurately for model fitting.

(Research previously published: Jieyu Zuo, Yuan Gao, Nadia Bou-Chacra, and Raimar Löbenberg, "Evaluation of the DDSolver Software Applications," BioMed Research International, vol. 2014, Article ID 204925, 9 pages, 2014. doi:10.1155/2014/204925.)

\section{Biorelevant Membranes as in vitro Models for the Evaluation of Drug Permeability}

Marie-Eve Leclaire ${ }^{1}$, Sarra Zaraa $^{1}$, Isabelle Marcotte $^{2}$ and Grégoire Leclair ${ }^{1}$

${ }^{1}$ Faculty of Pharmacy, Université de Montréal, Montreal, QC; ${ }^{2}$ Department of Chemistry, Université du Québec à Montréal, Montreal, QC

Purpose: Numerous high throughput assays have been developed to evaluate the permeability of drugs. PAMPA membranes, non cellular membranes impregnated with egg lecithin in dodecane, are less biorelevant than cellular Caco-2 assays, but which are more expensive and time-consuming. Therefore, there is a need to develop a high throughput biorelevant membrane to study the permeability of drugs through biological membranes.

Methods: A hydrophilic filter was covered with a polydopamine (pDA) cushion after an initial incubation period of $4 \mathrm{~h}$. Then, a phospholipid bilayer was deposited on the pDA cushion by liposomal fusion during $16 \mathrm{~h}$. Liposomes were composed of 1,2 dioleoylphosphatidylcholine (DOPC) and DC-cholesterol (DCChol) at different ratios and their size was measured by dynamic light scattering. Furthermore, the kinetic permeability of six compounds (propranolol, ketoconazole, ketoprofen, antipyrine, furosemide and hydrochlorothiazide (HCT)) was assayed.

Results: Liposomes size was over $300 \mu \mathrm{m}$ for all 
ratios. After permeability evaluation, the filter and the pDA were not a limiting factor of the diffusion. Increasing the DCChol proportion resulted in decreasing permeability because it conferred rigidity to the membrane. Kinetic evaluation of the permeability demonstrated that it remained constant over a period of $2 \mathrm{~h}$. The biorelevant membrane was able to statistically discriminate 5 out of the 6 drug standards based on their hydrophilicity as shown in the figure below $((\log \mathrm{P}), \mathrm{n}=40, \mathrm{IC} 95 \%)$.

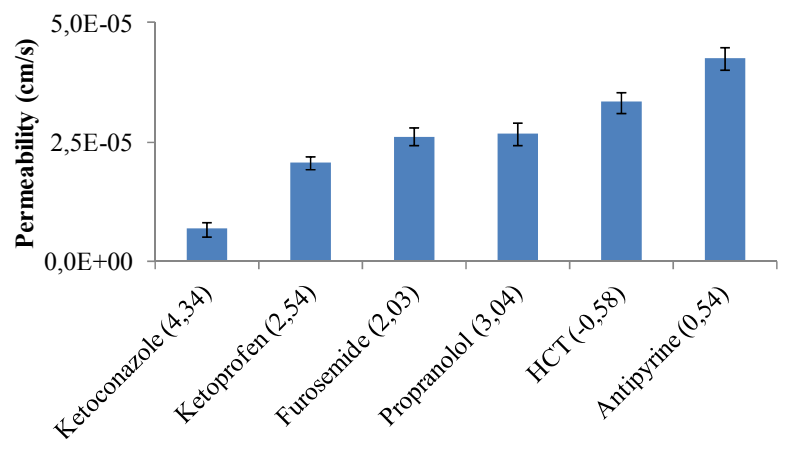

Conclusion: We developed a biorelevant membrane able to discriminate between compounds based on their hydrophilicity. This membrane has the potential to be more biologically representative than PAMPA and its preparation is faster compared to the Caco-2 assay. This membrane could be use to mimic different biological membrane such as the intestinal membrane and the blood-brain barrier. Research is ongoing to determine if this membrane could be a suitable model of the paracellular absorption of drugs.

\section{Surface Properties and Interactions of $\mathrm{pH}$ Sensitive Polymeric Nanoparticle Monolayers}

Lucie Giraud $^{1}$, Suzanne Giasson ${ }^{1,2}$

${ }^{1}$ Faculty of Pharmacy and ${ }^{2}$ Department of Chemistry, Université de Montréal, Montréal, QC, Canada

Purpose: We investigated core/shell polystyrene (PS)/ polyacrylic acid (PAA) nanoparticules (NPs) which are weakly charged and therefore sensitive to $\mathrm{pH}$ and ionic strength. These NPs made of PAA can undergo changes in volume, in surface potential and structure in response to changes in $\mathrm{pH}$ and/or ionic strength. Such NPs are promising materials for designing smart carriers whose surface properties (adhesion, permeablility) and interactions with the surrounding environment can be modulated in response to an external stimulus or signal.

Method: Supported self-assembled NPs monolayers were used as platform to investigate their interaction with the surrounding environment (solvent, surface), their surface properties (adhesion, friction) as well as mechanical properties (elasticity) by the use of molecular techniques such as Atomic Force Microscopy and Surface Forces Apparatus techniques.

Results: The NPs monolayers were irreversibly attached on amino-functionalized mica and silica substrates to prevent cleavage and/or slipping of the NPs at the polymer/substrate interface during force measurements. We have demonstrated that immobilized NPs maintained their responsiveness properties.

Conclusion: Normal and lateral forces between these supported NPs as a function of $\mathrm{pH}$ were measured and compared with different classes of solvated polymer-bearing surfaces in order to elucidate the role of the conformation, elasticity and electrostatic interaction of the polymer chains in controlling adhesion and friction between surfaces in aqueous media.

\section{Effect of Polymer Architecture on Hydrophobic Drugs Encapsulation and Release from Polymeric Nanoparticles}

Jimmy Faivre $^{1}$, Jean-Michel Rabanel ${ }^{1,2}{ }_{2}$ Patrice Hildgen $^{2}$, Xavier Banquy ${ }^{1}$

${ }^{1}$ Chaire de recherche du Canada sur les matériaux inspirés du vivant, Faculté de Pharmacie, Université de Montréal, Montréal, QC; ${ }^{2}$ Laboratoire de Nanotechnologie pharmaceutique, Faculté de Pharmacie, Université de Montréal, Montréal, QC, Canada

Purpose: Pegylated polyesters polymers have proved their usefulness in the preparation of nanosized drug delivery systems. However, the ability of the polymeric nanoparticles (NP) to encapsulate hydrophobic drugs ought to be optimized in order to reach the desired dose at the site of action. In order to improve the targeting efficiency, several aspects of NP preparation have been investigated. In this work, we hypothesized that this objective can be fulfilled by the addition of a controlled quantity of PEG chains on a polyester backbone, resulting in "comb" architecture. The performance of a series of PEG grafted PLA polymer (PEG-g-PLA) will be compared to diblock 
and PLA polymers. The final aim is to maximize drugs encapsulation levels with the view to investigate their effects on in vitro models of neurodegenerative diseases.

Method: PEG/PLA copolymers were synthetized as described elsewhere to yield a library of polyesters including diblock PEG-PLA, OH-g-PLA, and a series of PEG-g-PLA. Ibuprofen, anthracen and curcumin were encapsulated in NP prepared by nanoprecipitation in a PBS solution $(\mathrm{pH}$ 7.4). NP were slowly centrifugated then dialysed during $2 \mathrm{~h}$ against PBS, washed and concentrated via tangential filtration. NP were characterized for their size by DLS and zeta potential. Drug loading, drug encapsulation efficiency and drug release were determined by UV dosage. The stability of these NP were also investigated after the lyophilisation process.

Results: The size of the NP appeared to be non-drug dependant as the same time as drug encapsulation and loading by testing NP with different drug ratios. We demonstrated that the optimal drug loading for each polymer, as well as controlled release are dependent upon polymer architecture and PEG content. Finally, the NP ability to be redispersed in aqueous media after lyophilisation was found to be dependent on trehalose amount.

Conclusion: Drug loaded PLA/PEG nanoparticles were produced and characterized. Drug loading and efficiency were found to be satisfactory in regard to targeted applications. Encapsulation and release were found to have a dependency over PEG content and polymer architecture. The next step will be to investigate the dose effect relationship of optimized formulations on in vitro models of neurodegenerative disease with an oxidative stress component.

\section{Use of Heat-activated Thermosensitive Liposomes of Cisplatin (HTLC) for the Treatment Cervical Carcinoma}

Yannan N. Dou ${ }^{1}$, Jinzi Zheng ${ }^{2}$, Robert Weersink ${ }^{3}$, Naz Chaudary ${ }^{3}$, Warren D. Foltz ${ }^{2,3}$, David A. Jaffray $^{1,2,3}$, Christine J. Allen ${ }^{1,2}$

${ }^{1}$ University of Toronto, Toronto, ON; ${ }^{2}$ STTARR Innovative Center, Toronto, ON; ${ }^{3}$ Princess Margaret Hospital, Toronto, ON, Canada

Purpose: Cisplatin (CDDP) has been identified as the major chemotherapeutic agent for the treatment of cervical cancer, but the clinical use of CDDP is limited by its severe toxicities. In order to reduce systemic toxicities associated with CDDP, different advanced drug delivery systems such as liposomes have been investigated, including SPI-77 and Lipoplatin. Although these liposome formulations of CDDP have shown significantly higher tumor accumulation levels compared to free drug, their therapeutic advantages over free drug have been lacking due to insufficient release of the drug at the tumor site. Hence, we have developed a novel formulation of heat-activated thermosensitive liposomal cisplatin (HTLC) to increase the bioavailability of the drug in the tumor region via local heating.

Methods: The physico-chemical properties, pharmacokinetics and biodistribution of the HTLC were evaluated. By co-encapsulation of a gadolinium-based contrast agent, the release kinetics of HTLC was monitored in real-time using magnetic resonance imaging (MRI). Biodistribution of CDDP after every dose was assessed in female mice bearing subcutaneous ME-180 cervical tumor at one hour post-injection. Therapeutic efficacy of HTLC, free CDDP, and Lipoplatin was evaluated at their maximum tolerated dose (MTD).

Results: The phase transition temperature $\left(\mathrm{T}_{\mathrm{m}}\right)$ of HTLC was on average $41.5^{\circ} \mathrm{C}$. The size of the liposomes was shown to be stable over a 7 day incubation period at $4^{\circ} \mathrm{C}$. Under mild hyperthermia condition (i.e. $42^{\circ} \mathrm{C}$ ), approximately $90 \%$ of loaded drug was released in less than 5 minutes with minimal release at $37^{\circ} \mathrm{C}$. Using a two-compartment pharmacokinetic model, the HTLC exhibited a rapid distribution half-life followed by a long elimination half-life. In order to take advantage of the immediate heat-activated release mechanism and the pharmacokinetic profile of HTLC, a heating protocol that heats at the peak plasma drug concentration was adopted, where the tumor was preheated for 5 minutes before injection and heated for an additional 20 minutes after injection. This heating protocol was shown to effectively release the encapsulated gadoteridol causing contrast enhancement monitored by MRI. Results from tumor accumulation study showed that the overall tumor uptake levels for the HTLC groups were significantly higher than the corresponding free CDDP groups. The therapeutic efficacy of HTLC had further shown superior antitumor effect compared to free drug and Lipoplatin.

Conclusion: This HTLC formulation with immediate heat-activated mechanism offered extensive therapeutic advantage for the treatment of 
cervical cancer. Through the guidance of MRI, treatment strategies could be better optimized in the clinical setting.

Part of the data has been published in:

Y.N. Dou, J.Z. Zheng, W.D. Foltz, R. Weersink, N. Chaudary, D.A. Jaffray, C. Allen, Heat-activated thermosensitive liposomal cisplatin (HTLC) results in effective growth delay of cervical carcinoma in mice, Journal of Controlled Release 178 (2014) 6978.

\section{Part of the data has been presented in:}

1. $40^{\text {th }}$ Annual Meeting \& Exposition of the Controlled Release Society (CRS), July 2013.

2. 2013 Canadian Cancer Research Conference (CCRC), November 2013.

\section{Folate Receptor-Targeted Pluronic Nanoparticles for Ovarian Cancer Gene Therapy}

Samantha Shortall, Osama Madkhali and Shawn Wettig

School of Pharmacy, University of Waterloo, Waterloo, Ontario, Canada

Purpose: Non-viral gene delivery holds potential for safe delivery of exogenous genetic material as an alternative to viral delivery methods; however, nonviral vectors continue to suffer from lower transfection efficiency in comparison. Therefore, a rational, bottom-up design approach is required to develop an efficient and minimally toxic gene therapy formulation. Simultaneous physical characterization and transfection efficiency and cytoxicity assays are expected to advance rational design of this novel formulation so that safe and effective, targeted gene delivery can eventually be achieved in vivo

Method: A novel combination of dicationic, N,N'bis(dimethylalkyl)-alkane-diammoniumdibromide (m-s-m) gemini surfactants and Pluronic ${ }^{\circledR}$ block copolymers were used to develop self-assembling, soft nanoparticles for gene delivery. Nanoparticles were formed by adding Pluronic at varying concentrations to pVG.telRL plasmid DNA-gemini surfactant complexes at varying charge ratios, and assessed in physical and transfection assays. Transfection efficiency and in vitro cytotoxicity assays in OVCAR-3 cells were performed simultaneously with physical characterization of the resultant nanoparticles using dynamic light scattering and laser Doppler micro-electrophoresis to measure the particle size and zeta potential, respectively.

Results: Preliminary in vitro transfection studies in OVCAR-3 cells demonstrated a potential for efficient transfection with reduced cytotoxicity compared to a commercial control (Lipofectamine 2000). 14\% transfection was achieved with nanoparticles consisting of a DNA to gemini surfactant charge ratio of 1:5, combined with either Pluronic F87 at a concentration of twice its critical micelle concentration (CMC) or Pluronic F108 present at either its $\mathrm{CMC}$ or twice its CMC. Cell viability was $43 \%$ with $\mathrm{F} 87$ nanoparticles, and $48 \%$ with F108. These in vitro results corresponded with particle sizes below 200nm and low surface charges.

Conclusion: In order to enhance the performance of this promising system for gene delivery applications in vivo, folate-functionalized Pluronics can be used. This allows us to exploit the over-expression of folate receptor- $\alpha$ on the surface of human ovarian carcinoma cells; permitting increased transfection efficiency through targeted delivery to the cells of interest and faster internalization via receptormediated endocytosis. The hope is that there would also be less non-specific gene delivery; lowering toxicity in off-target cells.

\section{Tunable Growth Factor Delivery from Injectable Hydrogels for Central Nervous System Repair}

$\underline{\text { James Parker }}{ }^{1}$, Katarina Vulic ${ }^{1}$, Malgosia Pakulska ${ }^{1}$ and Molly Shoichet ${ }^{1,2}$

1. Department of Chemistry, University of Toronto, Toronto, Ontario, Canada; 2. Department of Chemical Engineering \& Applied Chemistry, University of Toronto, Toronto, Ontario, Canada

Controlled release of protein therapeutics has been achieved primarily by their encapsulation in polymeric, biodegradable nanospheres and microspheres. However, these strategies often suffer from low protein loading capacity and lost activity of the released proteins due to exposure to organic solvents and processing. To overcome these limitations, an affinity release hydrogel system was designed that takes advantage of the orthogonal association of Src homology 3 (SH3) and strong and weak SH3-binding peptides (SBP and WBP), which have dissociation constants of $10^{-5}$ to $10^{-7} \mathrm{M}$. Methyl cellulose (MC) hydrogel was covalently modified with either SBP or WBP using a Michael-type 
addition between MC-thiol and maleimide-peptide. Hydrogels comprised of a physical blend of SBP- or WBP-modified $\mathrm{MC}$ and hyaluronan (HA) demonstrated controlled release of SH3-FGF2 fusion protein. The release rate of the fusion protein was dependent on the strength of the SH3-peptide binding interaction. ${ }^{1}$ The broad applicability of this system was further demonstrated using chondroitinase $\mathrm{ABC}$ - an enzyme that has shown promise in the central nervous system. ${ }^{2}$ To investigate the release of two proteins at different rates from a WBP-modified hydrogel, pigment epithelium-derived factor (PEDF), which contains an HA-binding domain with $\mathrm{K}_{\mathrm{d}}=10^{-7} \mathrm{M}$, and SH3insulin-like growth factor-1 fusion protein (SH3IGF-1) have been expressed. The system is capable of delivering therapeutic proteins at tunable rates while maintaining bioactivity and thus is suitable for numerous applications.

Acknowledgements: We would like to express our gratitude to Dr. Karen Maxwell and Diane Bona for their advice and expertise during the cloning and purification of PEDF and SH3-IGF. We are grateful for support in the form of the NSERC Discovery Grant to Molly Shoichet, the NSERC CGS-M Scholarship to Jamie Parker, NSERC Vanier Scholarship to Malgosia Pakulska and Ontario Graduate Scholarship to Katarina Vulic.

1. Vulic K, Shoichet MS. Tunable growth factor delivery from injectable hydrogels for tissue engineering. J Am Chem Soc. 2012;134(2):882-885.

2. Pakulska MM, Vulic K, Shoichet MS. Affinitybased release of chondroitinase $\mathrm{ABC}$ from a modified methylcellulose hydrogel. $J$ Controlled Release. 2013;171(1):11-16. 


\section{Index}

\section{A}

Abadi, Kazem, 90

Aghazadeh-Habashi, Ali, 5, 47, 48, 88, 93, 97

Agu, Remigius U., 67

Ahmad, Mohammad S., 95

Akinwumi, Bolanle, 66

Al-awar, Rima, 3

Al-Awar, Rima, 22

Alharbi, Adnan J., 95

Alhowiriny, Tawfeq, 94

Ali Aghazadeh-Habashi, 47, 48, 88, 97

Allen, Christine J., 4, 34, 110

Almukainzi, May, 77, 98

Alomari, Mustafa, 5, 50, 79, 106

Aloui-Jamali, Moulay A., 63

Alsaad, Abdulaziz M. S., 91

Al-said, Mansour, 94

Alsharif, Shaker, 78

Al-Sohaibani, Mohammed, 94

Alwani, Saniya, 96

Al-Yahya, Mohammed, 94

Amorim de Oliveira, Michael, 3, 29, 99

Anderson, Hope D., 66

Anderson, Judy, 88

Arnason, J. T., 95

Asghar, Waheed, 48, 88, 92, 93

\section{B}

Badea, Ildiko, 77, 96

Badv, Maryam, 83

Bae, You Han, 4, 37

Bakaic, Emilia, 83

Banquy, Xavier, 6, 58, 64, 72, 78, 104, 109

Barralet, Jake, 61, 62, 101, 105

Basit, Abdul, 79, 106

Bedard, Leanne L., 6, 52

Belisle, Manon, 3

Benhaddou, Aicha, 80

Bennet, Andrew J., 90

Bergstrom, Dane, 69

Berkowitz, Steven A., 4, 32

Bernard, Florian, 5, 43, 63

Bhatia, Mickie, 3, 27

Black, W. Cameron, 3, 23

Bommana, Murali Mohan, 75

Bou-Chacra, Nadia, 107, 108

Braite, Drielly Cristina, 88

Brian Amsden, 1

Brian Foster, 1

Brkovic, Alexandre, 67

Bunker, Alex, 99

Burczynski, Frank J., 88, 89
C

C.-Gaudreault, René, 80

Cai, Zhongli, 69

Calon, Frédéric, 66

Campbell, Scott B., 82, 100

Campos Del'Orto, Juliana, 74

Cerruti, Marta, 105

Chatterjee, Sharmista, 6, 51

Chaudary, Naz, 110

Chen, Yufei, 72, 100

Cheng, Ryan, 103

Chitanda, Jackson M., 96

Clas, Sophie-Dorothee, 30

Cohen, Lucinda, 5, 42

Colin, Patrick, 2, 15, 16

Cote, Catherine, 103

Côté, Diane, 2, 14

Cox, Michael, 87

Crawford, Jason, 2, 12

Creaser, Colin, 5, 44

D

Daneshtalab, Noriko, 60

D'Aoust, P.M., 95

Das, Sanjoy Kumar, 3, 21

Davies, Neal M., 66, 89, 93

Dou, Yannan N., 110

Drager, Justin, 62

Duke, John, 97

E

Eliana Martins Lima, 99

Elizabeth Vadas, 28

Eloi, Sarah Montiel Gomes, 88

El-Tahir, Kamal, 94, 95

Endrenyi, Laszlo, 4, 33, 45

\section{F}

Fabio Garofolo, 42

Faivre, Jimmy, 109

Farah, C., 95

Farrer, Brian, 4, 39

Felhmann, Max, 2, 13

Foltz, Warren D., 110

Forest, Jean-Marc, 65

Foster, B. C., 1, 95

Fowke, Keith R., 100

Frankel, Grace E.C., 93

Friciu, Mihaela, 63 


\section{G}

Gaisford, Simon, 79, 106

Gao, Yuan, 107, 108

Gardner, David, 90

Garofolo, Fabio, 5, 42, 43

Gendreau, Isabelle, 80

Ghaffari, Mazyar, 87

Giasson, Suzanne, 58, 109

Giraud, Lucie, 109

Gong, Yuewen, 89

Gordon, Jacob, 87

Gosselin, Diane, 2, 11, 12

Gourgas, Ophélie, 72

Gu, Frank, 4, 34, 35

Gu, Jijin, 81

\section{H}

Haddadi, Azita, 70, 97, 102

Harvey, Edward, 62

Hegadoren, Kathleen, 5, 47

Hetu, Daniel, 2, 17

Hildgen, Patrice, 74, 104, 109

Ho, Emmanuel A., 3, 20, 72, 81, 100

Hoare, Todd, 1, 36, 82, 83, 84, 96, 100

Hossain, Mirza Akram, 80

\section{I}

Ibrahim, Fady, 68, 69

Imen Kahouli, 63

Ispas-Szabo, Pompilia, 105

\section{J}

Jaffray, David A., 110

Jahan, Sheikh Tasnim, 102

Jamali, Fakhreddin, 5, 48, 49, 88, 92, 93, 97

Johnson, Sarah, 91

Jutras, Martin, 63

\section{$\mathbf{K}$}

Kast, Juergen, 5, 43

Kaur, Kamaljit, 73

Khalid, Mohamed Nabil, 79

Khougaz, Karine, 41

Kim, Jenny Hanbi, 102

Klein, Agnes, 4, 31

Klein, Steven J., 2, 11

Koren, Gideon, 91

Kovacs, Elisabeth, 5, 50, 51

Krantis, A., 95

Krawetz, Roman, 5, 47, 48

Kumar, Rav, 5, 46

Kumar, Virender, 75

Kwong, Elizabeth, 1, 16
L

Lalloz, Augustine, 80

Larbi, Nassima, 105

Latreille, Pierre-Luc, 63, 64

Laurenti, Marco, 101

Lavasanifar, Afsaneh, 76

Lavoie, Cynthia, 2, 18

Le, Tien Cahn, 105

Leblond Chain, Jeanne, 73, 74, 76, 80, 99

Leclair, Grégoire, 1, 63, 65, 108

Leclaire, Marie-Eve, 108

Lefebvre, Marc, 2, 15, 67

Leoberto Costa Tavares, 99

Levesque, Jean-Francois, 53

Lévesque, Jean-François, 6, 53

Leyton, Jeffrey V., 69

Li, Amanda, 72, 73

Li, Ping, 89

Li, Xing-Cong, 95

Lim, Sue, 4, 31, 32

Lívia Palmerston Mendes, 99

Löbenberg, Raimar, 1, 77, 98, 107, 108

Luiz Alexandre Marques Wiirzler, 99

M

Madkhali, Osama, 111

Malhotra, Meenakshi, 60, 63

Manon Bélisle, 25

Marcotte, Isabelle, 108

Marinescu, Daniel, 63

Martinez, Stephanie E., 66, 93

Mateen, Rabia, 84, 96

Mateescu, Mircea Alexandru, 105

Max Fehlmann, 13

Mayer, Lawrence, 102

Mbarek, Amira, 73, 76

Mesnier, Aline, 76

Michel, Deborah, 77, 96

Midha, Ankur, 87

Mohamed Besheir, Hoda, 71, 72

Muhammad, llias, 95

Murphy, Andrea, 90

Musuku, Adrien, 3, 24, 67

$\mathbf{N}$

Narain, Ravin, 4, 35

$\mathbf{0}$

Obach, R. Scott, 6, 53, 54

Olah, Timothy, 65

Ormsby, Eric, 3, 26

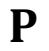

P.M. D'Aoust, 95

Pakulska, Malgosia, 111

114s | Conference 2014 
Pandher, Navpreet, 75

Panicucci, Riccardo, 4, 29, 30

Parker, James, 111

Peresypkin, Andrey, 3, 29

Petia Shipkova, 65

Piccirilli, Gianfranca, 67

Plourde, Kevin, 74, 99

Pollmann, André, 90, 91

Poorghorban, Masoomeh, 77

Pouliot, Roxane, 80, 81

Pouliot-Bérubé, Claudia, 81

Prakash, Satya, 60, 63

Prasit, Peppi, 2, 15

\section{$\mathbf{R}$}

Rabanel, Jean-Michel, 104, 109

Rafatullah, Sayed, 94

Rafiei, Pedram, 70

Raghuwanshi, Yogita, 73

Rahmouni, Miloud, 79

Raimar Loebenberg, 1

Randell, Amy, 60

Raymond, Martine, 80

Reilly, Raymond M., 69

Rima Al-awar, 22

Rivard, Georges-Étienne, 65

Robert Young, 1

Rodes, Laëtitia Sonia, 63

Roullin, V. Gaëlle, 6, 56

Roullin, Valérie Gaëlle, 57, 63, 71, 72, 74

\section{S}

Sadat, Sams Mohammad Anowar, 97

Saha, Shyamali, 60, 63

Samoylenko, Volodymyr, 95

Sant, Shilpa, 6, 57

Sarfraz, Muhammad, 98

Sarode, Ashish, 103

Savoie, Michelle, 2, 17

Sayre, Casey L., 66, 93

Schneider, Eric, 91

Selvaganapathy, P. Ravi, 100

Serrano-Wu, Michael, 3, 22

Shamsi, Saeideh, 90

Sharma, Supriya, 3, 19

Sheardown, Heather, 4, 39, 40

Sheikh, Zeeshan, 62

Shimotori, Takeshi, 103

Shin, Edward S., 102

Shipps, Gerald, 2, 18

Shoichet, Molly, 111

Shortall, Samantha, 111

Siddiqui, Arshad, 1, 23

Simard, Pierre, 80

Sivak, Olena, 68, 69

Sleno, Lekha, 44

Smeets, Niels M.B., 83

Soleymani Abyaneh, Hoda, 76

Soni, Vandana, 84
Squillante III, Emilio, 75

Squires, Krista, 60

St-Amour, Isabelle, 66

Standing, Joseph, 79, 106

Steinbach, Jill M., 40

Stinchcomb, Audra, 4, 38

St-Jean, Isabelle, 65

Strandman, Satu, 105

Strugari, Caitlin, 77, 78

Sugisawa, Kenji, 103

\section{T}

Tabrizian, Maryam, 60

Tamimi Marino, Faleh, 61, 101

Tardi, Paul, 102

Tearle, Howard, 87

Tehrani, Soudeh F., 71, 74

Théberge, Karine, 80

Tillman, Patrick, 67

Tomaro Duchesneau, Catherine, 60, 63

Trabelsi, Fethi, 3, 24, 25

Traore, Yannick, 72

Tsue, Shinichiro, 103

\section{V}

Vadas, Elizabeth, 3, 28

Vakili, Mohammad Reza, 76

Valtiner, Markus, 6, 56

Vandal, Milène, 66

Verrall, Ronald E., 96

Virgili, Jessica, 66, 67

Viricel, Warren, 4, 34, 73, 76, 99

Vulic, Katarina, 111

\section{W}

Waghmare, Kaetan, 60

Waite, Nancy, 91

Walker, Robin M., 6, 54

Walsh, Riley, 68, 69

Wang, Siyan, 88

Wang, Xiaotong, 89

Wasan, Kishor M., 68, 69, 87, 102

Weersink, Robert, 110

Wettig, Shawn, 111

Wong, Carly, 69

Worthen, David R., 103

Wrisley, Loren, 5, 50

\section{$\mathbf{X}$}

Xin, Baomin, 65

$\mathrm{Xu}$, Jinke, 105

\section{Y}

Yang, Jun, 100

Yang, Sidi, 4, 39, 81 
Yeung, Pollen K., 67

Young, Robert, 1

Yousaf, Muhammad, 95

Yu, Howe-Ming, 73

\section{$\mathbf{Z}$}

Zaraa, Sarra, 108

Zereshkian, Arman, 69, 70
Zhan, Fanglin, 76

Zhang, Helia, 88

Zhang, Huaifa, 61

Zheng, Jinzi, 110

Zheng, Joanna, 65

Zhu, Julian X.X., 105

Zuo, Jieyu, 107, 108

116s | Conference 2014 